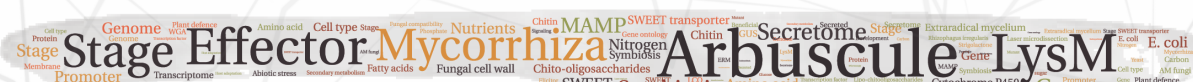

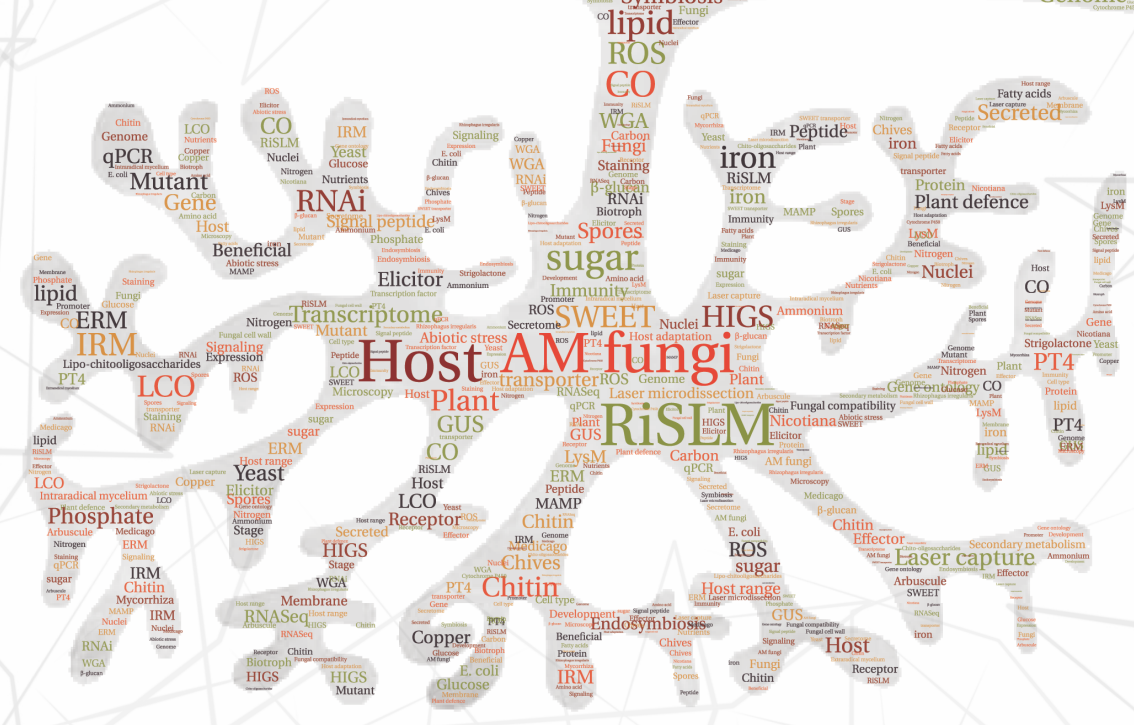

Trick or Treat:

Mechanisms Regulating Fungal Compatibility in Arbuscular Mycorrhizal Symbiosis

Insights from host- and stage-dependent transcriptome analyses

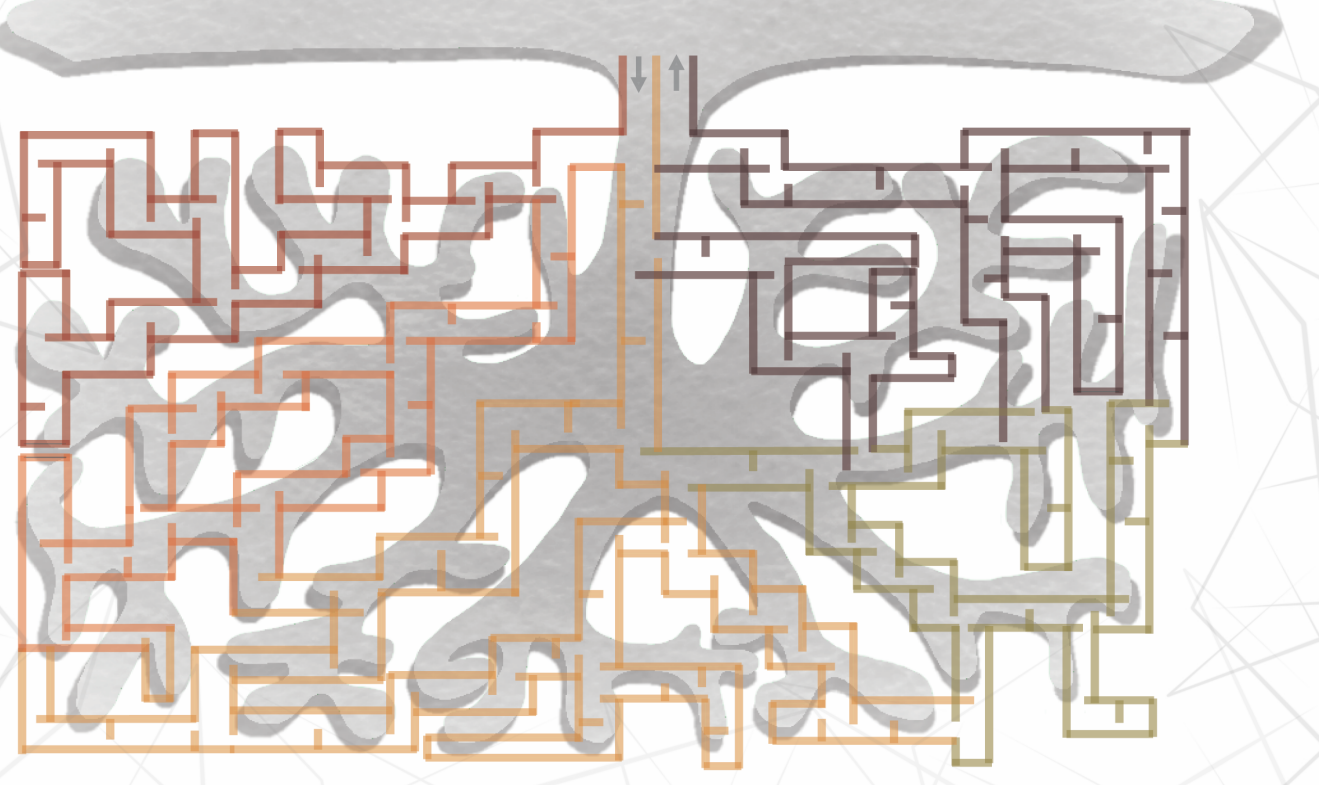

Tian Zeng

\section{INVITATION}

You are cordially invited to attend the public defence of my PhD thesis entitled:

Trick or Treat:

Mechanisms Regulating

Fungal Compatibility in

Arbuscular Mycorrhizal Symbiosis

on

Tuesday 10th Dec, 2019

1:30 p.m.,

in

the Aula of

Wageningen University,

Generaal Foulkesweg 1a,

Wageningen

Tian Zeng

tian.zeng@hotmail.com

\section{Paranymphs}

Rik Huisman

rik.huisman@wur.nl

Zhichun Yan

zhichun.yan@wur.nl 


\section{Trick or Treat:}

\section{Mechanisms Regulating Fungal Compatibility in Arbuscular Mycorrhizal Symbiosis}

Insights from host- and stage-dependent transcriptome analyses

\section{Tian Zeng}




\section{Thesis committee}

\section{Promotor}

Prof. Dr Ton Bisseling

Professor of Molecular Biology

Wageningen University \& Research

\section{Co-Promotor}

Dr Erik Limpens

Assistant professor, Laboratory of Molecular Biology

Wageningen University \& Research

\section{Other members}

Prof. Dr Thom Kuyper, Wageningen University \& Research

Prof. Dr Corné Pieterse, Utrecht University

Prof. Dr Natalia Requena, Karlsruhe Institute of Technology, Germany

Dr Matthieu Joosten, Wageningen University \& Research

This research was conducted under the auspices of the Graduate School of Experimental Plant Sciences. 


\title{
Trick or Treat: \\ Mechanisms Regulating Fungal Compatibility in Arbuscular Mycorrhizal Symbiosis
}

Insights from host- and stage-dependent transcriptome analyses

\section{Tian Zeng}

Thesis

submitted in fulfilment of the requirements for the degree of doctor at Wageningen University

by the authority of the Rector Magnificus

\author{
Prof. Dr A. P. J. Mol, \\ in the presence of the
}

Thesis Committee appointed by the Academic Board to be defended in public

on Tuesday 10 December 2019 at 1:30 p.m. in the Aula. 
Tian Zeng

Trick or Treat: Mechanisms Regulating Fungal Compatibility in Arbuscular Mycorrhizal Symbiosis

Insights from host- and stage-dependent transcriptome analyses 220 pages

PhD thesis, Wageningen University, Wageningen, NL (2019)

With references, with summary in English

ISBN: 978-94-6395-175-3

DOI: https://doi.org/10.18174/504202 


\section{CONTENTS}

Thesis outline $\quad 7$

Chapter 1. General introduction 11

Chapter 2. Host- and stage-dependent secretome of the arbuscular mycorrhizal fungus Rhizophagus irregularis $\quad 29$

Chapter 3. Host- and stage-dependent insights into the transcriptome of Rhizophagus irregularis DAOM197198 $\mathbf{6 1}$

Chapter 4. A LysM effector subverts chitin-triggered immunity to facilitate arbuscular mycorrhizal symbiosis $\mathbf{9 3}$

Chapter 5. A Medicago truncatula SWEET transporter implicated in arbuscule maintenance during arbuscular mycorrhizal symbiosis

Chapter 6. General discussion

References

181

Summary

209

Acknowledgements

213

Curriculum Vitae

216

List of publications 


\section{Thesis outline}

Most land plants rely on the symbiosis with arbuscular mycorrhizal (AM) fungi belonging to the Glomeromycotina subphylum to obtain sufficient nutrients from the soil. During this symbiosis, AM fungi are hosted inside inner root cortex cells, where they form highly branched hyphal structures, called arbuscules. This symbiosis is thought to have originated when plants first started to colonize the land and, due to the many benefits offered by this symbiosis, AM fungi are still able to intracellularly colonize over $80 \%$ of all land plants, making them arguably the most successful plant symbionts. How AM fungi manage to colonize and cooperate with such an extremely broad range of plants is a key question in AM biology. AM fungi must have evolved efficient ways to deal with the plant immune system and be able to adapt to their different host environments to ensure a beneficial interaction. However, the underlying mechanisms are still largely unknown. Most insight into the molecular aspects of this key symbiosis have been obtained from the plant side. However, the recent availability of the first genome sequence from the AM fungus Rhizophagus irregularis offered a great opportunity to obtain more insight into the fungal aspects of this symbiosis. Therefore, an important aim of this thesis was to obtain more cell-specific insight into the transcriptome of both the fungus and the plant at different stages of the interaction and to compare the fungal response in diverse host plants. This allowed me to identify novel key regulators involved in AM symbiosis, mainly focusing on effector biology and nutrient exchange with the host.

In Chapter 1, I give a general overview of the AM symbiosis and focus especially on signalling events that control different stages of the interaction.

In Chapter 2, I studied the secretome of Rhizophagus irregularis, to identify putative effector proteins that contribute to its broad host range. Secreted effector proteins are well known from pathogenic fungi to play a key role in subverting the host immune system and to reprogram the plant to allow fungal colonization. Therefore, I compared the AM fungal secretome of $R$. irregularis in three evolutionary divergent hosts, namely Medicago truncatula, Nicotiana benthamiana and Allium schoenoprasum. I identified both commonly expressed effector candidates as well as potential effectors that are expressed in a host-dependent manner. By 
applying laser microdissection coupled with RNA sequencing in Medicago, I further dissected the expression pattern of putative effectors into different stages, including arbuscules, intraradical mycelium, extraradical mycelium and germinating spores. These approaches collectively resulted in an expression atlas of the fungal secretome and allowed me to pinpoint potential key effectors in AM symbiosis.

In Chapter 3, I used gene-ontology enrichment analyses for a broader characterization of the host- and stage-dependent fungal transcriptome data to get more insight into the response of the fungus to different host plants. As providing (mineral) nutrients to the host plant in exchange for sugars and fatty acids is one of the most pronounced roles of AM fungi, I highlight candidate key transporters for such nutrients at different stages of the interaction. Furthermore, I discuss the host-dependent transcriptional responses to shed light on fungal adaptation to the different host environments and propose a potential key effect of host identity on fungal (secondary) metabolism.

In Chapter 4, I studied the role of one of the highest intraradically expressed effector candidates in all hosts, encoding a LysM domaincontaining effector (RiSLM). To elucidate the role of RiSLM in AM symbiosis, we used a combination of molecular, biochemical and genetic approaches. This showed that RiSLM can bind chitin(oligomers), protect fungal cell walls from plant chitinases and suppress chitin-triggered immune responses in the plant. Host-induced silencing of RiSLM further indicated a positive role for this effector in the symbiosis. These results revealed a conserved role for LysM effectors in both pathogenic as well as symbiotic fungal interactions with plants.

In Chapter 5, the role of a potential plant sugar exporter in AM symbiosis was studied. Although sugars have long been proposed to be transferred from the host to AM fungi, the mechanisms by which these sugars are transported by the plant to the fungus have remained elusive. By analysing the stage-specific transcriptome data, we identified a single SUGARS-WILL-EVENTUALLY-BE-SECRETED (SWEET) transporter, called $M t S W E E T 1 b$, that was significantly expressed in arbuscule-containing cells of Medicago. MtSWEET1b was shown to transport glucose and to localize to the peri-arbuscular membrane. Overexpression of MtSWEET1b increased fungal colonization levels, while interference with its activity via 
overexpression of dominant-negative versions caused an early collapse of arbuscules. These results suggest a (redundant) role for MtSWEET1b in the transport of glucose across the peri-arbuscular membrane to maintain arbuscules for a healthy mutually beneficial symbiosis.

In Chapter 6, I summarize our current insights and discuss potential mechanisms that contribute to the broad host-range of AM fungi in light of effector biology and reciprocal nutrient transfer. 
CHAPTER 1

General introduction 


\section{CHAPTER 1}

Arbuscular mycorrhiza (AM) fungi, affiliated to the fungal Glomeromycotina subphylum, can form a symbiotic interaction with the majority $(>80 \%)$ of land plants (S., Smith and Read, 2008; Spatafora et al., 2016). They can benefit their hosts in multiple ways. The primary advantage of AM fungi is that they facilitate host uptake of limiting mineral nutrients such as phosphate, nitrogen, or microelements from the soil by developing extensive extraradical hyphal mycelium, which expand the absorption surface of the host root system (S., Smith and Read, 2008). Furthermore, AM fungi provide protection against plant pathogens, for example by improving host fitness, competing for colonization sites with pathogens or the activation of systemic acquired resistance. Additionally, they affect the microbiome surrounding the roots and improve soil structure. In return for such benefits, AM fungi receive considerable amounts (up to $20 \%$ ) of photosynthetic carbohydrates (Jakobsen and Rosendahl, 1990) and/or lipids from the plant (Keymer et al., 2017; Luginbuehl et al., 2017; Jiang et al., 2017; Bravo et al., 2017; Helber et al., 2011). In fact, AM fungi have become obligate biotrophs that fully depend on their host plants to complete their lifecycle.

The AM symbiosis is predicted to have originated about 450 million years ago, by the same time that plants started to colonize the land (Remy et al., 1994). This, together with the extremely broad host-range of AM fungi, indicates that both plant and fungal partners have co-evolved to maintain the symbiosis. The mechanisms behind the extremely high compatibility of AM fungi are still largely unknown. On one hand, symbiotic signalling triggered by mycorrhizal (myc) factors is required to efficiently colonize host plants (Fig.1). In addition, a successful AM symbiosis requires a strict coordination of nutrient exchange by both partners. Although insight into the fungal components that control the symbiosis has lagged behind compared to those of the plant, major progress has been made in recent years through the availability of the first AM fungal genome and transcriptome (Tisserant et al., 2012; Tisserant et al., 2013; Lin et al., 2014a). It is becoming clear that, on top of myc-factor signalling, AM fungi use effector proteins to achieve a successful symbiosis (Lin et al., 2014a; Tisserant et al., 2013; Tisserant et al., 2012; Kloppholz et al., 2011). Here, I will give an overview of the AM symbiosis with a focus on symbiotic signalling, effector biology and nutrient exchange in $A M$ symbiosis. 


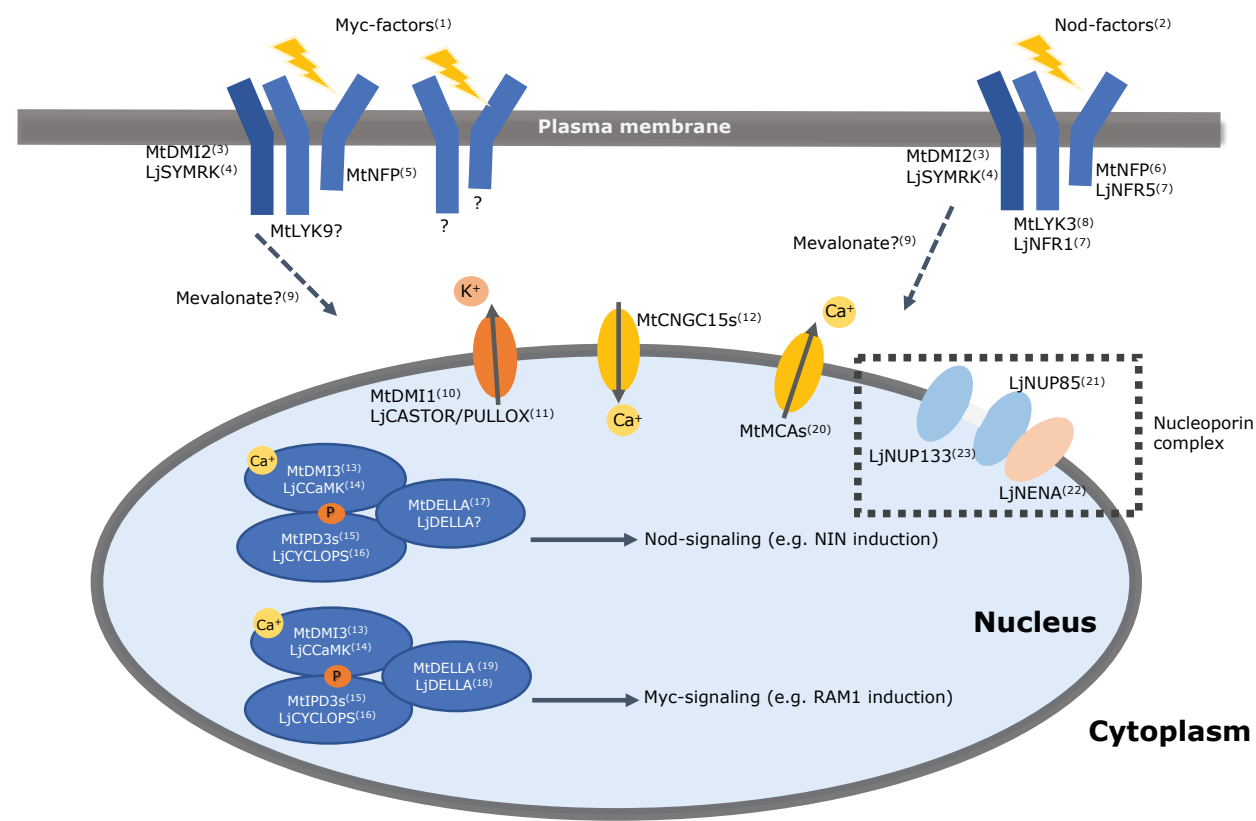

Figure 1. Common symbiotic signaling pathway. This signaling pathway is essential for a successful AM symbiosis as well as for the rhizobium-legume symbiosis. To initiate the signaling, Myc-factors (1) and Nod-factors (2) are perceived by plant LysM receptors $(5,6,7,8)$. These are thought to form a complex with MtDMI2/LjSYMRK $(3,4)$, which interacts with a 3-hydroxy-3methylglutaryl coenzyme A reductase 1 (MtHMGR, 9). This interaction generate mevalonate as a potential secondary messenger to the nucleus (9). Activation of the CSSP eventually leads to nuclear calcium spiking. This is mediated by nuclear envelope located potassium $(10,11)$, calcium channels (12) and a calciumtransporting ATPase (20). In addition, components of the nucleoporin complex are required for calcium spiking $(21,22,23)$. Calcium spiking is decoded by the calmodulin/calcium-dependent protein kinase MtDMI3/LjCCaMK $(13,14)$, leading to phosphorylation and activation of transcription factors MtIPD3/MtIPD3Like/ LjCYCLOPS $(15,16)$. MtIPD3s or LjCYCLOPS interacts with Medicago or Lotus DELLA proteins to initiate downstream signaling of rhizobium symbiosis (17) or AM symbiosis $(18,19)$.

References 1. (Maillet et al., 2011; Genre et al., 2013); 2. (Lerouge et al., 1990; Roche et al., 1991b,a; Schultze et al., 1992); 3. (Endre et al., 2002); 4. (Stracke et al., 2002); 5. (Maillet et al., 2011); 6. (Arrighi et al., 2006); 7. (Radutoiu et al., 2003); 8. (Limpens et al., 2003); 9. (Venkateshwaran et al., 2015); 10. (Ané et al., 2004); 11. (Kistner et al., 2005; Riely et al., 2007); 12. (Charpentier et al., 2016); 13. (Lévy et al., 2004); 14. (Tirichine et al., 2006) ;15. (Messinese et al., 2007; Ovchinnikova et al., 2011; Horváth et al., 2011; Jin et al., 2018; Lindsay et al., 2019); 16. (Yano et al., 2008); 17. (Singh et al., 2014; Jin et al., 2016); 18. (Pimprikar et al., 2016); 19. (Floss et al., 2013); 20. (Capoen et al., 2011); 21. (Saito et al., 2007); 22. (Groth et al., 2010); 23. (Kanamori et al., 2006). 


\section{CHAPTER 1}

\section{Signalling in AM symbiosis}

Development of AM symbiosis is coordinated by both partners through mutual signal exchange, structural rearrangements and (post-) transcriptional or (post-)translational regulation. There are several distinct stages in AM symbiosis. First, AM fungi and plant hosts exchange signals to pre-adapt for the symbiosis (pre-contact signalling). Consequently, fungal hyphae reach the plant root surface and, upon formation of a hyphopodium, penetrate into the epidermis (epidermal penetration, Fig. 2a, b) (Gutjahr and Parniske, 2013). Fungal hyphae subsequently spread inside the root either inter-cellularly or intra-cellularly (intraradical colonization, Fig. 2a, b) and eventually reach the inner cortical cell layer. There, fungal hyphae can enter into cells where they differentiate to a highly branched, tree-like structure, called an arbuscule (arbuscule formation, Fig. 2a, b). Arbuscules are surrounded by a specialized host membrane in which many nutrients transporters are located to allow the efficient nutrient transfer between both partners. Therefore, arbuscules are considered as the heart of the symbiosis (Luginbuehl and Oldroyd, 2017).

\section{Pre-contact signalling}

AM symbiosis is initiated by signal exchange of both the plant and fungal partners before physical contact. The best studied plant signals are strigolactones. Strigolactones are phytohormones regulating shoot and root branching. In addition, root-secreted strigolactones are perceived by AM fungi and stimulate fungal mitochondrial activity and fungal respiration, ultimately promoting spore germination and hyphal branching (Akiyama et al., 2005; Besserer et al., 2006). This process eventually recruits $A M$ fungi into the rhizosphere and raises the chance of fungal contact with the root. In many, if not all, host plant species genes regulating strigolactone biosynthesis, such as DWARF27 and strigolactone transporters such as PDR, are upregulated under phosphate starvation to enhance strigolactone secretion (Liu et al., 2011; Kretzschmar et al., 2012). This suggests that plants have evolved a very efficient strategy to overcome phoshate/nutrient stress by recruiting AM fungi as partners.

Recent forward genetic studies have revealed a role for novel symbiotic signals from the host in pre-contact signalling, such as $\mathrm{N}$-acetylglucosamine 
(GlcNAc) and karrinkin-like molecules. Mutants of a GlcNAc transporter, called nope1, are severely impaired in early steps of fungal colonization, particularly hyphopodia formation in rice and maize (Nadal et al., 2017). Treating AM spores with either wild type or nope1 root exudates induced distinct transcriptomic changes in the fungus, suggesting that rootsecreted GlcNAc (or related molecules) is a plant derived signal that is perceived by the fungus (Nadal et al., 2017). In rice, mutation of the $a / \beta$ fold hydrolase DWARF14-LIKE (D14L) caused similar severe defects at an early stage of the symbiosis (Gutjahr et al., 2015). d14l mutants showed almost complete absence of hyphopodia, with consequently impaired root colonization. $D 14 L$ encodes a component of an intracellular receptor complex which perceives among others smoke compounds called karrikins. However, the perceived signals relevant for AM symbiosis are likely not karrikins, as application of karrikins was not able to rescue defects in the $d 14$ l mutant (Gutjahr et al., 2015). Absence of D14L impaired most root transcriptional responses to germinating spore exudates, implying a defect in symbiotic signalling at a very early stage in line with the lack of hyphopodia in $d 141$.

In response to plant signals such as strigolactones, AM fungi release fungal signals that are perceived by the host to facilitate fungal colonization.

(a)
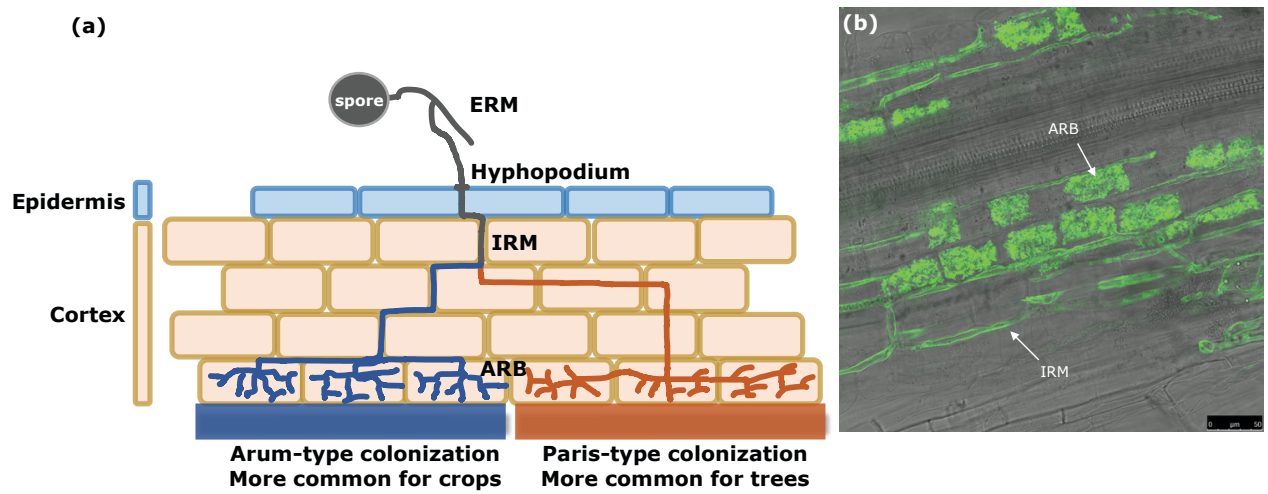

Figure 2. Different developmental stages during AM symbiosis. (a) Schemetic picture showing different developmental stages of AM symbiosis, including germinating spores, extraradical mycelium (ERM), intraradical mycelium (IRM), and arbuscule (ARB). Blue and yellow IRM and ARB indicate arum-type or paris type colonization, respectively. (b) Arbuscules (ARB) and intraradical mycelium (IRM) developmented in Medicago truncatula in symbiosis with Rhizophagus irregularis. WGA Alexa 488 was used to visualize fungal structures. Scale bar $=50 \mu \mathrm{m}$. 


\section{CHAPTER 1}

The best studied fungal signals are chitin-based compounds such as lipochitooligosaccharides (LCOs) or short-chain chitooligosaccharides (COs) (Maillet et al., 2011; Genre et al., 2013). Myc-LCOs are structurally similar to rhizobial Nod-LCOs, called Nod factors (Maillet et al., 2011). They also share functional commonalities such as promotion of lateral root formation and activation of a so-called common symbiotic signalling pathway (Figure 1) leading to nuclear calcium spiking and symbiotic gene expression. Nod-LCOs are perceived by plant Lysin-motif (LysM) receptor(s) (kinases) such as LYK3/NFP or NFR1/NFR5 in the model legumes Medicago truncatula and Lotus japonicus, respectively (Limpens et al., 2003; Oláh et al., 2005; Broghammer et al., 2012; Radutoiu et al., 2003). Therefore, it is hypothesized that LysM receptors are also required to perceive Myc-LCOs. In support of this, non-sulfated myc-LCOs failed to trigger lateral root formation in the Medicago $n f p$ mutant (Maillet et al., 2011). However, considerable mycorrhization is still retained in the Medicago $n f p$ mutant, suggesting that functionally redundant receptors are involved in myc-LCO perception (Maillet et al., 2011). In non-nodulating plant species such as rice, myc-factors were shown to be perceived by the LysM receptor kinase OsCERK1(Zhang et al., 2015a; Miyata et al., 2014). OsCERK1 represents the ortholog of Arabidopsis AtCERK1 and function as a co-receptor for chitin-triggered immune responses by interacting with co-receptors OsCEBiP in case of rice and AtLYK4/5 in Arabidopsis (Shimizu et al., 2010; Miya et al., 2007; Cao et al., 2014). Reduced mycorrhization was observed in the rice cerk1 mutant but not cebip, indicating that OsCERK1 is a receptor involved in both symbiosis and immunity (Zhang et al., 2015a; Miyata et al., 2014). Apart from LCOs, short-chain COs (mainly $\mathrm{CO} 4$ and $\mathrm{CO} 5$ ) are another class of myc-factors. Secretion of fungal myc-COs was shown to be stimulated by strigolactone treatment and application to roots induced symbiotic responses, such as calcium spiking, in different hosts (Genre et al., 2013). A recent study identified Medicago (MtLYK9, MtLYR4) and Lotus (LjLys6) LysM domain containing receptor-like kinases as receptors for longer chain COs (such as CO7/8) to trigger defense responses (Bozsoki et al., 2017). Mutation of these receptors impaired chitin-triggered immune responses, but still allowed a successful, although slightly reduced, AM symbiosis (Bozsoki et al., 2017; Gibelin-Viala et al., 2019). Therefore, it is proposed that different LysMreceptor kinase containing complexes are required to perceive symbiotic LCOs and short chain CO's in Medicago and Lotus (Bozsoki et al., 2017). 
In line with this, a double mutant of MtNFP and MtLYK9 showed a much more severe reduction in mycorrhization levels compared to the single mutants (Giles Oldroyd, personal communication).

\section{Epidermal penetration and intraradical colonization}

Signal perception by plant and fungal partners stimulates root growth and hyphal branching, respectively, to facilitate physical contact of both partners. After physical contact, the AM fungal hyphal tip can form a special structure, called a hyphopodium, from which the fungus can penetrate the root epidermis. The formation of a hyphopodium is likely controlled by pre-contact signalling, as the $\mathrm{N}$-acetylglucosamine (GICNAC) transporter mutant nope1 and karrinkin-receptor mutant $d 14$ I are both impaired in hyphopodium formation (Nadal et al., 2017; Gutjahr et al., 2015). After hyphopodia develop, the plant host rearranges its cytoskeleton to be ready for hyphal penetration. A specialized structure called pre-penetration apparatus (PPA), composed of ER cisternae, actin and microtubules is assembled in the epidermal cell shortly before hyphal penetration, providing a path for fungal hyphae to cross the cell (Genre et al., 2005; Genre et al., 2008). Genomic and transcriptomic studies revealed that AM fungi lack enzymes to degrade plant cell walls (Tisserant et al., 2012; Tisserant et al., 2013). Therefore, penetration of the plant cell wall during fungal colonization is dependent on enzymes of the host.

Formation of the PPA and subsequent epidermal crossing requires the activation of the common symbiotic signaling pathway (Fig. 1). Consistently, root colonization is impaired in mutants of the CSSP. Ectopic expression of an auto-active version of LjCCaMK is sufficient to induce the formation of PPA-like structures in the absence of the fungus (Takeda et al., 2012), suggesting that PPA formation is controlled by the CSSP. Active symbiotic signalling is likely required for fungal infection throughout the root. In line with this, calcium spiking was observed in cortical cell nuclei upon fungal penetration (Sieberer et al., 2012).

Once past the epidermis, AM fungi show a range of growth strategies inside the root that range from the so-called "Arum-type" to the "Paristype" (Fig. 2a). In Arum-type infection, the fungal hyphae grow in the intercellular space between cortex cells. After fungal hyphae reach inner cortical cells, the hyphae penetrate into the cells to form arbuscules (Fig. 


\section{CHAPTER 1}

2a). This is regarded as the most common strategy in cultivated crop plants, which have been studied the most (Dickson, 2004). In contrast, in paris-type infection, which occurs more in tree species, fungal hyphae penetrate and cross cortical cells, forming coils and/or arbuscule-like side branches (Fig. 2a). Arum- or Paris-type infection is dependent on (the combination of) plant and fungal genotype (Dickson, 2004). However the mechanisms that determine the infection strategy are still unknown.

\section{Arbuscule development}

After fungal hyphae reach inner cortical cells, they penetrate into the cells to form arbuscules. The first step of arbuscule development highly resembles PPA formation, followed by the development of an arbuscular trunk and subsequent further higher order branches. Arbuscules become surrounded by a host membrane, called the peri-arbuscular membrane, where many membrane transporters are located to allow efficient nutrient transfer (Ivanov et al., 2012). Formation of arbuscules and their lifetime is therefore tightly regulated by the host. Typically, arbuscules are relatively short-lived and after 2-5 days they are degraded, after which the cortex cell can potentially be colonized again (Kobae and Hata, 2010).

Because calcium spiking was observed in cortical cells during fungal colonization (Sieberer et al., 2012), it is thought that the common symbiotic signalling pathway also controls arbuscule formation. However, both Ljnena and Ljsymrk mutants, are still able to develop healthy arbuscules, despite the fact that epidermal infection is dramatically reduced (Stracke et al., 2002; Demchenko et al., 2004; Groth et al., 2010). This indicates that LjSYMRK/MtDMI2 and LjNENA are only essential for fungal colonization of the epidermis, but not for arbuscule development in cortical cells. However, mutations of LjCCaMK/MtDMI3 completely block arbuscule development, suggesting that there must be regulatory elements parallel to LjNENA and LjSYMRK that function upstream of LjCCaMK/MtDMI3, at least in cortical cells.

Activated MtDMI3/LjCCaMK in turn phosphorylates proteins to activate downstream signalling. A key phospho-target of CCaMK is CYCLOPS in Lotus. Mutation of CYCLOPS impairs arbuscule development while epidermal colonization is retained (Yano et al., 2008). CYCLOPS is a transcription factor that binds to the promoter of the transcription factor 
RAM1 and induces its transcription (Pimprikar et al., 2016). RAM1 is a GRAS type transcription factor. Mutation of RAM1 leads to a severe defect in arbuscule development, resulting in only a trunk or bird-feet structure in several plant species (Park et al., 2015; Rich et al., 2015; Pimprikar et al., 2016). Therefore it was hypothesized that RAM1 is essential for hyphal branching during arbuscule development (Pimprikar et al., 2016). Induction of RAM1 expression by CYCLOPS can be boosted by co-expression of a dominant form of DELLA transcriptional regulators, which are key repressors of gibberelin (GA) signalling (Pimprikar et al., 2016). DELLA proteins are considered to be essential for arbuscule development, as a della1/della2 double mutant in Medicago largely fails to form arbuscules (Floss et al., 2013). Consistently, application of exogenous GA blocks arbuscule development in Lotus, while application of the GA antagonist PAC restores arbuscule development in cyclops mutants (Pimprikar et al., 2016). Restoration of arbuscule development in cyclops mutants or in presence of GA can also be achieved by ectopic expression of RAM1 driven by a constitutive promoter (Pimprikar et al., 2016). These results, together with the observation that PAC treatment induces RAM1 expression, places RAM1 downstream of a transcriptional regulatory complex formed by CCaMK, CYCLOPS and DELLA (Pimprikar et al., 2016). RAM1 was further shown to interact with its close homolog, REQUIRED FOR ARBUSCULE DEVELOPMENT (RAD1, Park et al., 2015; Xue et al., 2015), and to the DELLA INTERACTING PROTEIN1 (DIP1, Yu et al., 2014), likely forming higher order heteromeric complexes to control arbuscule branching (Pimprikar et al., 2016).

Recent promoter binding studies revealed an important role for AM-induced AP2 transcription factors in Medicago and Lotus, downstream of RAM1. The Lotus AP2 transcription factor CBX1 and its Medicago homolog WRINKLED transcription factor WRI5a were shown to directly bind to the promoters and induce the expression of arbuscule-specific nutrient transporter genes, such as MtSTR/MtSTR2 thought to be involved in fatty acid (FA) transport and the arbuscule-specific phosphate transporter PT4 (Jiang et al., 2018; Xue et al., 2018). Interestingly, transactivation of MtWRI5a also induced expression of MtRAM1, and vice versa (Jiang et al., 2018). This indicates that RAM1 and WRI5a are forming a positive regulatory loop in arbuscule-containing cells, to ensure sufficient expression level of downstream genes (Jiang et al., 2018). LjCBX1 and MtWRI5 were shown 


\section{CHAPTER 1}

to be able to bind to cis-regulatory elements, including MYCS and/or an AW-box, which are enriched in the promoter of various arbuscule-specific genes that control the bidirectional exchange of phosphorus and FAs (Xue et al. , 2018). In addition to phosphate transporters such as LjPT4, LjCBX1 was shown to induce LjRAM2 by promoter binding (Xue et al., 2018). RAM2 encodes a glycerol-3-phosphate acyltransferase which functions to transform C16:0-FA and glycerol-3-phosphate to C16:0 monoacylglycerol (C16:0 $\beta$-MAG) during lipid biosynthesis. Mutation of RAM2 leads to the early degeneration of arbuscules similar to mtpt4 mutants, likely due to a defect in the supply of FAs to the fungus.

Considering the function of arbuscules in nutrient transfer, various transporters need to be precisely targeted to the peri-arbuscular membrane. For instance, the best know symbiotic nutrient transporter MtPT4, as well as the proton pump MtHA1 which generates the proton gradient to energize MtPT4, and the proposed lipid transporters MtSTR and MtSTR2, are localized only on the fine branches of the peri-arbuscular membrane (PAM) (Javot et al., 2007; Wang et al., 2014a; Krajinski et al., 2014; Zhang et al., 2010). This indicates that exocytic pathways are precisely regulated in arbuscule-containing cells. Recent studies suggested the importance of a symbiosis-dedicated exocytic pathway in arbuscule formation in AM symbiosis (Zhang et al., 2015b; Ivanov et al., 2012; Huisman et al., 2016). Knockdown lines of symbiosis-dedicated vesicle SNAREs VAMP721d/e or target-SNARE SYP132a in Medicago were shown to be severely hampered in arbuscule development (Ivanov et al., 2012; Huisman et al., 2016). Furthermore, an exocyst component MtEXO70i was shown to localize to the tip region of the arbuscule fine branches, where it interacts with MtVAPYRIN (Zhang et al., 2015b). MtVAPYRIN contains an N-terminal VAMP-associated protein (VAP)/major sperm protein (MSP) domain and a C-terminal ankyrin-repeat domain (Pumplin et al., 2010). Knock-out/down mutants of MtVAPYRIN are unable to form arbuscules and are also impaired in epidermal passage (Murray et al., 2011). Medicago exo70i mutant showed limited incorporation of STRs into the peri-arbuscular membrane, which may cause the early degenerating arbuscule phenotype in the exo70i mutant (Zhang et al., 2015b). The fact that Mtvapyrin mutants show a more severe defect in arbuscule formation suggests that additional EXO70 members may be involved (Zhang et al., 2015b). In addition to a regulated exocytosis 
pathway, the specific localization of nutrient transporters is also tightly regulated by the precise timing of transcription in arbuscule-containing cells. It was reported that a transient reorientation of secretion from plasma membrane to PAM occurs during arbuscule development (Pumplin et al., 2012). This is supported by the fact that expression of a membrane localized phosphate transporter MtPT1 driven by the arbuscule-specific MtPT4 promoter is sufficient to localize MtPT1 to the fine branches of the peri-arbuscular membrane, whilst MtPT1 protein driven by the $35 \mathrm{~S}$ promoter (which is switched off upon arbuscule formation) showed plasma membrane localization (Pumplin et al., 2012). These observations suggest a substantial remodeling of membrane trafficking in arbusculecontaining cells.

\section{Nutrient transfer in AM symbiosis}

AM fungi are well known for their ability to absorb mineral nutrients by their highly developed extraradical hyphal network and to transfer these to the plant host. Phosphate transfer is generally considered as the most important function of AM symbiosis. Inorganic phosphate can be taken up by high affinity phosphate transporters located in the AM fungal extraradical mycelium (Harrison and Buuren, 1995; Maldonado-Mendoza et al., 2001). The phosphate can be converted into polyphosphate to be stored in the vacuole and transported to intraradical mycelium through aquaporin-mediated water flow (Kikuchi et al., 2016). Arbuscules are considered to be the main site for phosphate transfer to the hosts. However, how phosphate can be unloaded to the peri-arbuscular space is currently still not known, although several potential phosphate transporters were identified in genomic or transcriptomic studies (Tisserant et al., 2012, 2013; Chapter 3). Phosphate can then be taken up by the plant from the peri-arbuscular space via arbuscule-specific phosphate transporters such as MtPT4, that are fuelled by a proton gradient generated by a periarbuscular membrane located proton pump (Javot et al., 2007; Wang et al., 2014a; Krajinski et al., 2014). In addition to their role in inorganic phosphate uptake, AM fungi were proposed to utilize organic phosphate such as phytate either by secreting acid phosphatases or by cooperating with phosphate solubilizing bacteria associated with fungal hyphae (Zhang et al., 2016; Sato et al., 2015). In addition to phosphate transporters, 


\section{CHAPTER 1}

a few ammonium and nitrate transporters were identified in $A M$ fungi (López-Pedrosa et al., 2006; Pérez-Tienda et al., 2011; Calabrese et al., 2016). Such transporters are thought to enable AM fungi to take up nitrogen from the soil and to supply it to the host, mainly in the form of ammonium or ammonia (Fellbaum et al., 2012; Govindarajulu et al., 2005).Consistently, many plant ammonium transporters are induced upon AM symbiosis in various plant species (Guether et al., 2009a; Guether et al., 2009b; Koegel et al., 2013; Kobae et al., 2010) of which some have been shown to be located at the peri-arbuscular membrane, such as SbAMT3;1 (Koegel et al., 2013) or GmAMT4.1 (Kobae et al., 2010). However, it should be noted that arbuscule-specific ammonium transporters may also convey other functions. For instance, Medicago AMT2;3 does not show any ammonium transport activity, but instead is involved in controlling arbuscule maintenance under low nitrogen conditions (Breuillin-Sessoms et al., 2015). Therefore AMT2;3 may function as a transceptor.

In addition to mineral nitrogen, it was reported that AM fungi can also utilize (peptide- and protein-bound) amino acids from the environment as organic nitrogen/nutrient source (Cappellazzo et al., 2008). Mycorrhization was shown to modify the amino acid composition in the host, by facilitating the uptake of neutral or positively-charged amino acids rather than negatively-charged amino acids (Whiteside et al., 2012). In addition, an AM fungal peptide transporter was shown to be upregulated in intraradical stage of the symbiosis (Belmondo et al., 2014). Besides a role in amino acid supply to the plant, such amino acid or peptide transporters could also be involved in uptake of amino acids or peptides from the host. Interestingly, many amino acid transporters and oligopeptide transporters were also discovered in the genome of the obligate biotrophic pathogenic rust fungi (Duplessis et al., 2011). Whether acquiring amino acids or peptides from the hosts is a wide-spread mechanism in plant-microbe interactions remains to be determined. AM symbiosis was also reported to be able to provide potassium to the host, which is likely mediated by arbuscule-specific potassium transporters such as SiHAK10 in tomato (Liu et al., 2019). Last, AM symbiosis was shown to increase other metal ions such as iron nutrient levels in host plants, although the underlying transport mechanisms are not understood yet (Garcia et al., 2017; Chorianopoulou et al., 2015). 
AM fungi are obligate biotrophs that fully rely on the plant host for supply of energy sources as well as several micronutrients. Isotope tracer studies have indicated that hexoses, especially glucose, are transferred from the host to AM fungi (Solaiman and Saito, 1997; Shachar-Hill et al., 1995; Bago et al., 2003; Bago et al., 2000). Consistently, a high affinity monosaccharide transporter called RiMST2 (MONOSACCHARIDE TRANSPORTER 2) was identified in the AM fungus Rhizophagus irregularis (Helber et al., 2011). In addition to glucose, RiMST2 was suggested to transport cell wall monosaccharides. RiMST2 is predominantly expressed in arbuscule-containing cells and intraradical hyphae and is required for proper development of arbuscules and the level of fungal colonization (Helber et al., 2011). Whether sugars are actively transported across the PAM to be taken up by the fungus is still unknown. Recently, SWEET transporters have been identified in Arabidopsis thaliana as major sugar efflux transporters (Chen et al., 2012). SWEET transporters were also reported to be involved in plant-microbe interactions. For example, a SWEET gene in Arabidopsis was reported to be induced by pathogenic bacterial effectors to acquire glucose from the hosts (Chen et al., 2010). Furthermore, in the $\mathrm{N}_{2}$-fixing symbiosis, a SWEET transporter in Medicago was proposed to mediate sugar efflux to rhizobium bacteria (Kryvoruchko et al., 2016). Interestingly, during the progression of AM colonization, several SWEET transporters were induced in Solanum tuberosum (ManckGötzenberger and Requena, 2016). In Chapter 5, we identified a single SWEET protein in Medicago that is significantly induced in arbusculecontaining cells and studied its role in AM symbiosis.

The view that hexoses are the main carbon source for AM fungi changed recently when it was found that AM fungi lack a type I fatty acid (FA) synthase gene (FasI) in their genome. FasI is essential to synthesize palmitic acid (C16:0), although type II mitochondrial FAS genes which function to produce octanoic acid are present in the AM genome (Wewer et al., 2014; Keymer et al., 2017; Luginbuehl et al., 2017; Jiang et al., 2017). This indicated that AM fungi are in fact FA auxotrophs that need to obtain FA precursors from their hosts. In line with this it was observed that many lipid biosynthesis genes are induced in arbuscule-containing cells (Gaude, Bortfeld, et al., 2012; Gaude, Schulze, et al., 2012). In Medicago, overexpression of lipid biosynthesis genes such as pyruvate kinase (MtPK), ketoacyl-ACP synthase II (MtKAS II), ketoacyl-acyl carrier 


\section{CHAPTER 1}

protein $(A C P)$ reductase (MtKAR), enoyl-ACP reductase $I$ (MtENR I), and acyl-ACP thioesterase $B$ (MtFatM) led to higher mycorrhization levels, whilst silencing of those genes decreased mycorrhization and affected arbuscule development (Jiang et al., 2017). Similarly in Lotus, mutation of genes involved in lipid biosynthesis impaired arbuscule development. These genes include DIS, which encodes a $\beta$-keto-acyl ACP synthase I (KASI) required to synthesize FA chain from $C 4$ to $C 16$, FatM/FatB which encodes an acyl-ACP thioesterase required to terminate FA synthesis and RAM2 which encodes a glycerol-3-phosphate acyltransferase 6 (GPAT6) required to synthesize $\beta$-mono-acyl glycerol (sn2-MAG) (Keymer et al., 2017; Keymer and Gutjahr, 2018). After expression of a FatB gene of Umbellularia californica in Medicago, which terminates FA elongation after 12 carbons, tracer studies clearly detected 12:0 FA inside the fungus (Jiang et al., 2017; Luginbuehl et al., 2017). This provided direct evidence that the host transfers lipids to AM fungi (Jiang et al., 2017; Luginbuehl et al., 2017). Isotope profiling in Lotus led to a similar conclusion (Keymer et al., 2017). Lipids are likely exported to the peri-arbuscular space by two plant half-size ABCG transporters such as MtSTR and MtSTR2 (Zhang et al., 2010; Jiang et al., 2017; Keymer et al., 2017). However, it remains elusive how AM fungi take up lipids from the peri-arbuscular space.

\section{AM effectors}

Plants growing in nature are surrounded by an array of beneficial to detrimental microbes. A key feature which allows plants to survive in such an environment is the ability to distinguish their friends from foes. Plants can sense microbes by recognizing microbe-associated molecular patters (MAMPs) through cell-surface pattern recognition receptors (PPRs) that trigger defence reactions. One of the best characterized fungal MAMPs is chitin, which is a key part of the cell wall of fungi. Chitin consists of longchain $\beta-1,4$-linked polymers of $\mathrm{N}$-acetylglucosamine (GICNAC). Chitooligosaccharides (COs), particularly long-chain GlcNAc-oligomers such as hexamers (CO6) to octamers ( $\mathrm{CO} 8$ ), are efficiently perceived by plant immune LysM receptors such as AtCERK1 and AtLYK4/5 to induce defence responses in plant (Cao et al., 2014). Structural studies showed that the immune LysM receptor AtCERK1 requires at least six GICNAc residues for receptor dimerization to trigger downstream immune signalling in 
Arabidopsis (Liu et al., 2012).

Perception of MAMPs by PPRs triggers plant immune signalling, leading to rapid plant defence responses such as generation of reactive oxygen species (ROS), phosphorylation of mitogen-activated protein kinases, induction of defence related genes, and secretion of fungal cell wall targeted hydrolases. Therefore, mechanisms to evade or suppress host immunity are essential for all microbes to colonize plants. In plant pathogenic microbes secreted effector proteins are key players to evade or suppress plant immunity. Such effectors can function in the apoplast or they can be translocated into the host cells to exert their function (Lo Presti et al., 2015).

During colonization, the plant apoplast represents the first line of the battle field. Hydrolases such as chitinases are secreted to the apoplast to degrade fungal cell walls and to release COs which can be perceived by plant chitin receptors to trigger more defence responses. To counteract host chitinases, plant pathogens like Cladosporium fulvum have evolved effectors carrying chitin-binding LysM domains such as ECP6 (de Jonge et al., 2010). ECP6 is able to outcompete plant chitin receptors to bind fungal cell wall derived COs thereby impairing the activation of immune responses (de Jonge et al., 2010). LysM effectors were further identified in a wide range of pathogenic fungi (Marshall et al., 2011; Mentlak et al., 2012; Lee et al., 2014; Takahara et al., 2016; Kombrink et al., 2017), indicating that LysM effectors represent a common strategy to impair chitin-triggered immune responses. Other common fungal cell wall components that are perceived as PAMPs are $\beta$-glucans (Fesel and Zuccaro, 2016). The symbiotic fungus Piriformaspora indica was recently shown to secrete a $\beta$-glucan binding effector, called PiFGB1, which alters cell wall composition and suppresses glucan triggered immunity (Wawra et al., 2016). One of the key responses upon PAMP perception by plants is the production of reactive oxygen-species (ROS) by membrane-bound or apoplastic peroxidases to kill the invaders. Pathogenic fungi are known to overcome ROS stress by producing apoplastic effectors. For example, Ustilago maydis effector Pep1 can inhibit the activity of POX12, which is a peroxidase essential to generate ROS in the host (Doehlemann et al., 2009; Hemetsberger et al., 2012). Consistently, deletion of Pep1 severely impaired pathogenicity of Ustilago maydis (Doehlemann et al., 2009). Additional functions of apoplastic effectors include the inhibition 


\section{CHAPTER 1}

of plant proteases that function in plant immunity or the inactivation of plant derived antimicrobial compounds.

In addition to apoplastic effectors, microbes also secrete effectors that translocate into the host cell to facilitate infection. These effectors usually have highly variable targets, that function in the immune process through hormonal signalling, MAPK cascades, vesicle trafficking, regulation of transcription and alternative splicing and chromatin remodelling (Liu et al., 2014; Gimenez-Ibanez et al., 2014; Plett et al., 2014; Kloppholz et al., 2011; Huang et al., 2017; Song et al., 2015; Kong et al., 2017; Wang and Wang, 2018; Bi and Zhou, 2017). However, how such effectors are able to enter the host cells is still largely elusive (Petre and Kamoun, 2014).

Recent studies have shown that also beneficial fungi use effector proteins to deal with the host immune system. In the ectomycorrhizal fungus Laccaria bicolor, an effector called MiSSP7 was shown to be able to interact with the JAZ6 protein in Populus trichocarpa (Plett et al., 2011; Plett et al., 2014). This interaction is essential to stabilize JAZ6, leading to the repression of jasmonic acid induced immunity (Plett et al., 2014). Recent genomic and transcriptomic studies revealed several hundreds of effector-coding genes in the genome of AM fungi Rhizophagus irregularis (Sędzielewska Toro and Brachmann, 2016; Tisserant et al., 2013; Tisserant et al., 2012; Lin et al., 2014a). The first identified AM effector, called SP7, was shown to interact with the host transcription factor ERF19 to suppress host immunity in Medicago (Kloppholz et al., 2011). Furthermore, expression of SP7 in the rice blast fungus Magnaporthe oryzae prolonged its biotrophic phase in the plant, suggesting that SP7 may play a role in maintaining the biotrophic interaction in AM symbiosis (Kloppholz et al., 2011). By transcriptomic analyses, a strigolactoneinduced effector called RiSIS1 was discovered in $R$. irregularis (Tsuzuki et al., 2016). Host induced gene silencing of RiSIS1 resulted in reduced colonization, indicating an positive role for RiSIS1 in AM symbiosis, although the underlying molecular mechanism is currently not known (Tsuzuki et al., 2016). Interestingly, an effector carrying a single LysM domain was also revealed among the strigolactone-induced AM genes (Tsuzuki et al., 2016; Sędzielewska Toro \& Brachmann, 2016; Chapter 2 ). Considering the role of LysM effectors in plant-pathogen interactions and the fact that AM fungi need to suppress chitin-triggered immunity 
during colonization, the AM LysM effector was functionally characterized in Chapter 4.

\section{Conclusion and perspectives}

It has become clear that communication between AM fungi and their plant hosts involves a plethora of signals to ensure that AM fungi are treated as friends and to prevent the interaction from becoming parasitic. Such signals include LCOs, short chain COs, secreted effector proteins, secondary metabolites as well as nutrients. The exact identity of many of these signals and their mode of action are expected to be identified in the future. This is especially facilitated by the availability of the first AM fungal genome sequences. To increase the resolution of current transcriptomics approaches more cell-specific transcriptome approaches may greatly facilitate the identification of key components at various stages of the interaction. Identifying the molecular players and their mode of action will be important to address one of the most important questions in AM biology, namely: what determines the symbiotic efficiency of different plant-fungus interactions? The molecular basis for such differences are still largely unknown. Unraveling this key aspect of AM symbiosis will also be pivotal to fully exploit the potential of AM fungi for sustainable agriculture. 


\section{CHAPTER 2}

\section{Host- and stage-dependent secretome of the arbuscular mycorrhizal fungus Rhizophagus irregularis}

Tian Zeng ${ }^{1}$, Rens Holmer ${ }^{1,2}$, Jan Hontelez ${ }^{1}$, Bas te Lintel-Hekkert ${ }^{3}$, Lucky Marufu $^{1}$, Thijs de Zeeuw ${ }^{1}$, Fangyuan $\mathrm{Wu}^{1}$, Elio Schijlen ${ }^{3}$, Ton Bisseling ${ }^{1}$, Erik Limpens ${ }^{1}$

1. Laboratory of Molecular Biology, Department of Plant Sciences, Wageningen University \& Research, Droevendaalsesteeg 1 , Wageningen, 6708 PB, the Netherlands

2. Bioinformatics group, Department of Plant Sciences, Wageningen University \& Research, Droevendaalsesteeg 1, Wageningen, 6708 PB, the Netherlands

3. Bioscience, Plant Research International, Wageningen University \& Research, Droevendaalsesteeg 1, Wageningen, 6708 PB, the Netherlands

Corresponding author: Erik Limpens, erik.limpens@wur.nl

This chapter has been published in the Plant Journal as a cover paper. https://doi.org/10.1111/tpj.13908 


\section{CHAPTER 2}

\section{Abstract}

Arbuscular mycorrhizal fungi form the most wide-spread endosymbiosis with plants. There is very little host-specificity in this interaction, however host preferences as well as varying symbiotic efficiencies have been observed. We hypothesize that secreted proteins (SPs) may act as fungal effectors to control symbiotic efficiency in a host-dependent manner. Therefore, we studied whether AM fungi adjust their secretome in a host- and stage-dependent manner to contribute to their extremely wide host-range. We investigated the expression of SP-encoding genes of Rhizophagus irregularis in three evolutionary distantly-related plant species, Medicago truncatula, Nicotiana benthamiana and Allium schoenoprasum. In addition we used laser microdissection in combination with RNAseq to study SP expression at different stages of the interaction in Medicago. Our data indicate that most expressed SPs show roughly equal expression levels in the interaction with all three host plants. In addition, a subset shows significant differential expression depending on the host plant. Furthermore, SP expression is controlled locally in the hyphal network in response to host dependent cues. Overall, this study presents a comprehensive analysis of the $R$. irregularis secretome, which now offers a solid basis to direct functional studies on the role of fungal SPs in AM symbiosis.

\section{Introduction}

The vast majority of all land plants establish an endosymbiosis with arbuscular mycorrhizal (AM) fungi belonging to the fungal subphylum Glomeromycotina (Spatafora et al., 2016). This endosymbiosis, which originated 450 million years ago, provides a number of benefits to plants such as improved uptake of scarce nutrients (especially $\mathrm{P}$ and $\mathrm{N}$ ) and water from the soil, and protection against various biotic and abiotic stresses (Smith and Read, 2008). Individual AM fungi can colonize a large number of host plants, which indicates that (in general terms) there is a lack of host-specificity, although host preferences have been reported (Helgason et al., 1998; Vandenkoornhuyse et al., 2003; Torrecillas et al., 2012; van der Heijden et al., 2015). AM fungi establish extensive below ground networks interconnecting different plant species and most plant roots are 
colonized by multiple AM fungi. This extremely broad host-range implies that AM fungi must have highly efficient mechanisms to intracellularly colonize so many plants. Despite the absence of clear host-specificity, it has become clear that there can be substantial functional diversity in the benefits provided by AM fungi, which depends on both plant host and fungal genotype in combination with environmental conditions (Lee et al., 2006; Gosling et al., 2016; van der Heijden et al., 2015; Koch et al., 2017; Sawers et al., 2017). Such functional diversity has been observed between different AM fungal species as well as within species or even within fungal individuals (Sanders, 2010). However, the molecular basis for the observed differences in symbiotic efficiency in plant-fungus combinations is still largely unknown. Recently, it has become clear that AM fungi secrete effector proteins that manipulate functions (Kloppholz et al., 2011; Tsuzuki et al., 2016) in the host and we hypothesize that they may be key factors to control symbiotic efficiency and/or host range. Therefore, more insight is required in the expression profile of potential effector encoding genes during the interaction with different host plants.

To establish a functional AM symbiosis continuous signal exchange between both partners is needed. This dialogue starts when fungal spores/hypha perceive plant signals such as flavonoids, hydroxy fatty acids and strigolactones which stimulate germination, hyphal growth and branching or hyphopodium formation (Nadal and Paszkowski, 2013). In turn the fungus produces signal molecules to facilitate its entry into the root. The best studied fungal signals are lipo-chitooligosaccharides and short chitooligosaccharides (Maillet et al., 2011; Genre et al., 2013). These essential signals are perceived by LysM-domain receptor kinase complexes which activate a highly conserved signaling cascade, also called the common symbiotic signaling pathway (Gutjahr and Parniske, 2013; Oldroyd, 2013; Zipfel and Oldroyd, 2017).

In addition to the chitin-derived signal molecules, AM fungi also secrete an array of (small) proteins that may act as effector proteins. Effector proteins are extensively studied in the field of phytopathology, where pathogenic fungi or oomycetes as well as bacteria use effector proteins to manipulate host cells to facilitate successful infection; changing plant structure, suppressing innate immune responses or altering host metabolism. Such effectors can act in the apoplast or they are translocated to the host cells to exert their function in particular subcellular 


\section{CHAPTER 2}

compartments (Giraldo and Valent, 2013; Chaudhary et al., 2014; Lo Presti et al., 2015; Doehlemann et al., 2014). Recently, it has become clear that also symbiotic fungi employ effectors in a similar manner (Plett and Martin, 2015). The first AM effector, called SP7, was identified from Rhizophagus irregularis (Kloppholz et al., 2011). SP7 was reported to translocate to the nucleus of plant cells, where it interacts with the pathogenesis-related ERF19 transcription factor in Medicago truncatula, to suppress defense responses. Genomic approaches have since revealed the secretomes of several AM fungi. The genome sequence of the $R$. irregularis strain DAOM197198 identified several hundreds of potentially secreted proteins many of which may act as effectors (Tisserant et al., 2013; Lin et al., 2014a; Sędzielewska Toro and Brachmann, 2016). Several of the genes encoding these predicted secreted proteins are highly induced in planta (Tisserant et al., 2013) and by the application of the synthetic strigolactone analog GR24 (Tsuzuki et al., 2016). Additional predicted secretomes of Rhizophagus clarus (Sędzielewska Toro and Brachmann, 2016), Gigaspora margarita and Gigaspora rosea (Tang et al., 2016; Kamel et al., 2017b) suggest that a subset of putative AM effectors is conserved among AM species, in addition to many lineage specific secreted proteins (Kamel et al., 2017a,b).

Studies on effector expression in pathogenic systems has shown that many effectors are expressed in a host-, stage- and tissue-specific manner (Doehlemann et al., 2014; Guyon et al., 2014). Host-dependent cues have been shown to induce distinct sets of effector genes in the endophyte Piriformospora indica, correlating with host-dependent colonization strategies (Lahrmann et al., 2013). This raises the question whether AM effectors may also be used in a host- and stage-dependent manner to contribute to the extremely wide host-range or to control symbiotic efficiency. Therefore, we investigated the transcriptome of $R$. irregularis DAOM197198 in three evolutionary distantly related plant species, Medicago truncatula (Medicago), Nicotiana benthamiana (Nicotiana) and Allium schoenoprasum (Chives), and focussed especially on its predicted secretome, representing putative AM effectors. In addition we used laser microdissection in combination with RNAseq to study the secretome at different stages of the symbiotic interaction in Medicago. Our results reveal that distinct sets of putative effector genes are expressed in extraradical mycelium, intraradical hyphae and in arbuscules. The vast 
majority of the expressed effector genes are expressed equally in all three plant species, but in addition a set of host-dependent effector candidates were identified. These putative effector genes are induced in response to local cues determined by the plant. These results support the hypothesis that AM effectors may control symbiotic efficiency in a host-dependent manner and offers a comprehensive set of candidate AM effectors for future functional studies.

\section{Materials and Methods}

\section{Growth systems (plant and fungal material)}

Medicago (Medicago truncatula) Jemalong A17 seeds were surfacesterilized according to Limpens et al. (2004) and vernalized at $4^{\circ} \mathrm{C}$ for 2 days. Seeds were germinated at $20^{\circ} \mathrm{C}$ for 1 day before transfer into pots. Chives (Allium schoenoprasum) and Nicotiana (Nicotiana benthamiana) seeds were sterilized by $20 \%$ bleach for $15 \mathrm{~min}$, followed by 8 washes with sterile demineralized water. Rhizophagus irregularis DAOM197198 was maintained as monoxenic carrot (Daucus carota) root culture (Lin et al., 2014a). To prepare inoculum for pot experiments, $R$. irregularis DAOM197198 was propagated on Chives for 2 months in 1:1 sand/ clay mixture. Medicago, Chives and Nicotiana were inoculated by mixing $1 / 5(\mathrm{v} / \mathrm{v})$ inoculum with $1: 1$ sand/clay $(2-5 \mathrm{~mm}$; Jongkind B.V., Aalsmeer, The Netherlands) into new $14 \mathrm{~cm} \varnothing$ pots. Medicago plants were watered by $1 / 2$ Hoagland nutrition solution with full nitrogen $\left(5 \mathrm{mM} \mathrm{Ca}\left(\mathrm{NO}_{3}\right)_{2}, 5 \mathrm{mM}\right.$ $\mathrm{KNO}_{3}, 1 \mathrm{mM} \mathrm{MgSO}_{4}, 50 \mu \mathrm{M}$ NaFeEDTA, $20 \mu \mathrm{M} \mathrm{KH}_{2} \mathrm{PO} 4,10 \mu \mathrm{M} \mathrm{H}_{3} \mathrm{BO}_{3}, 0.2 \mu \mathrm{M}$ $\mathrm{Na}_{2} \mathrm{MoO}_{4}, 1 \mu \mathrm{M} \mathrm{ZnSO}, 2 \mu \mathrm{M} \mathrm{MnCl}_{2}, 0.5 \mu \mathrm{M} \mathrm{CuSO}_{4}, 0.2 \mu \mathrm{M} \mathrm{CoCl}, 12.5 \mu \mathrm{M}$ $\mathrm{HCl}$ and $500 \mu \mathrm{M} \mathrm{MES}$ ) twice per week. Chives and Nicotiana were watered by $1 / 2$ Hoagland solution with full nitrogen and demineralized water once each per week. 3 biological replicates were used for each plant species. All plants were grown under a $16 \mathrm{~h}$ daylight regime at $21^{\circ} \mathrm{C}$ and harvested 7 weeks post inoculation.

To test whether expression of SPs is controlled locally in a shared hyphal network, a double-compartment pot system was set up. Pots $(17,5 \mathrm{~cm} \varnothing)$ were separated into a central compartment and an outer compartment by a $45 \mu \mathrm{m}$ bore diameter mesh. Only in the central (nurse) compartment, $1 / 5(\mathrm{v} / \mathrm{v})$ propagated inoculum was added. In order to establish a hyphal 


\section{CHAPTER 2}

network that connects plants in both compartments, 10 chives plants or 3 Medicago plants were transferred into the central compartment and grown for 6 weeks at $21{ }^{\circ} \mathrm{C}$ as described above. After 6 weeks, 4 Medicago seedlings were transferred into the outer compartment. 3 biological replicates were used. All plants were harvested after another 4 weeks.

To test whether SP7 is more actively expressed in hyphae that are closely associated to root which we refer to as "rhizosphere ERM" compared to ERM that is further away from the root ("soil ERM"), Medicago roots were carefully harvested from pots 6 wpi. Roots and soil were rinsed by demi water separately to collect ERM. Water containing ERM was then subsequently passed through 3 layers of mesh, $500 \mu \mathrm{M}, 120 \mu \mathrm{M}$ and $45 \mu \mathrm{M}$. ERM were collected from the $120 \mu \mathrm{M}$ and $45 \mu \mathrm{M}$ mesh using forceps under a stereomicroscope. Four Medicago plants from 2 pots were used as 1 replicate. 3 biological replicates were used for RNA extraction and qPCR analysis.

\section{Laser Microdissection}

7 week-old Medicago roots infected by $R$. irregularis were cleaned and fixed by Farmer's fixative ( $75 \%$ ethanol and $25 \%$ acetic acid) for 16 hours at $4{ }^{\circ} \mathrm{C}$. Fixed roots were dehydrated in an ethanol series $75 \%(30 \mathrm{~min})$, $85 \%$ (30min) and $100 \%(3 \times 30 \mathrm{~min})$. Steedman wax was made by mixing $90 \%$ polyethylene glucol 400 distearate and $10 \%$ 1-hexadecanol at $65^{\circ} \mathrm{C}$ for 2 hours. Dehydrated roots were first infiltrated by ethanol/steedman wax (1:1) mixture overnight, followed by 3 times incubation in $100 \%$ steedman wax for 2 hours at $40^{\circ} \mathrm{C}$. Finally the roots were transferred to an aluminium plate to solidify. $14-\mu \mathrm{m}$-thick sections were cut by a microtome (Leica RJ2035/RM2255) and stretched on sterile water containing 40mM DTT on a UV-treated $4 \mu \mathrm{m}$ PEN-membrane slide (Leica). Steedman wax was removed by 3 times washing in $100 \%$ ethanol for $3 \mathrm{~min}$. The slides were air dried just before laser microdissection. Arbuscule cells and intraradical mycelium cells were collected using a Leica LMD7000 laser microdissection microscope. All microdissected cells were collected into the cap of a $0.5 \mathrm{ml}$ eppendorf tube containing $70 \mu \mathrm{L}$ RLT buffer (Qiagen RNeasy Micro kit) containing 40mM DTT. In total $\sim 2000$ cells were collected for each arbuscule replicate and $\sim 10000$ cells were collected for each intraradical mycelium replicate. 


\section{RNA isolation}

For all plant species colonized by mycorrhiza, whole root RNA was isolated using the Qiagen plant RNA mini kit according to manufacturer's protocol. Isolated RNA samples were quality checked using an Agilent 2100 Bioanalyzer at BGI (Hongkong).

For samples collected by laser microdissection, the quality of the RNA was first checked for cells leftover on each slide after microdissection. Therefore RNA was isolated and analysed using the Agilent RNA 6000 Pico kit on an Agilent 2100 Bioanalyzer. Next microdissected samples from slides that showed RIN $>4$ values with discernible $28 \mathrm{~S} / 18 \mathrm{~S}$ peaks in the leftover samples were selected and used for RNA isolation using the Qiagen RNeasy Microkit according to manufacturer's protocol. After RNA isolation, $2 \mu \mathrm{L}$ of each RNA sample was reverse transcribed using iScript cDNA Synthesis kit (Bio-Rad) and pre-amplified using Bio-Rad SsoAdvanced PreAmp Supermix. The performance of the amplified cDNA samples was checked by qPCR using mycorrhiza-induced marker genes, as described below. RNA samples from the best 3 replicates of each cell type were amplified using the SMART-Seq v4 Ultra Low Input RNA Kit (Clontech) according to manufacturer's instructions. The number of amplification cycles (11-15 cycles) for each sample was adjusted based on the amount of RNA determined by bioanalyzer and qPCR analyses.

\section{Library prep and sequencing}

For the plant species experiment, sequencing libraries were made using TruSeq RNA Sample Prep Kit v2 (Illumina) and sequenced on a Illumina HiSeq2000 platform (paired-end, 90bp $\times 2$ ) at BGI. For laser microdissected samples, sequencing libraries were made using ThruPLEX DNA-seq Kit (Rubicon genomics) and sequenced on a Illumina HiSeq 2500 platform (paired-end, $125 \mathrm{bp} \times 2$ ) at the NGS facility of Bioscience, Wageningen University \& Research.

\section{RNAseq analyses}

Sequence reads were mapped to the $R$. irregularis genome (Lin et al., 2014a) with corrections for effector gene models (Table S1) using CLC genomics workbench 10.0.1 (Qiagen). Length fraction and similarity fraction was set to 0.9 during mapping and only unique mapped reads 


\section{CHAPTER 2}

were considered in the analysis. All other parameters were set as default. TPM (transcripts per million, Wagner, Kin, and Lynch 2012) and differential expression analyses were generated by CLC genomic workbench 10.0.1. To filter the data, only genes with more than 100 total mapped reads in the combined whole root samples or or developmental stages were considered. RNASeq data of all samples were tested for saturation using NOISeq package in R 3.4.2 (Tarazona et al., 2015). PCA analysis was done using the DESeq2 package in R 3.4.2 (Love et al., 2014). To calculate effectors enriched in each plant species and fungal developmental stages, a cut-off of fold change $>4$ with an FDR $p<0.05$ was used.

\section{Annotation of secreted proteins}

Signal peptides were predicted using SignalP 2.0/4.1 (Petersen et al., 2011; Nielsen and Krogh, 1998) with default settings, and SPs were called according to the criteria in Lin et al. (2014). Blast2Go 4.0.7 was used to generate functional annotation of secreted proteins. In detail, blast searches were performed using the NCBI database. InterPro Scan function in Blast2GO was used to generate domain information. Mapping and annotation were all performed with default parameters in Blast2GO (Conesa et al., 2005).

\section{Mycorrhizal quantification}

Trypan blue staining was used to visualize mycorrhization in all samples (Chabaud et al., 2006). Mycorrhized roots were first cleared by potassium hydroxide $(\mathrm{KOH})$ for $20 \mathrm{~min}$ at $90{ }^{\circ} \mathrm{C}$ followed by 2 washes with demineralized water. Trypan blue staining solution was prepared by combining $25 \mathrm{ml} 2 \%(\mathrm{w} / \mathrm{v})$ trypan blue stock solution and 1 l Lactoglycerol solution $(300 \mathrm{ml}$ Lactic acid, $300 \mathrm{ml}$ Glycerol and $400 \mathrm{ml}$ double-distilled water). Cleared roots were submerged in trypan blue staining solution and stained at $90^{\circ} \mathrm{C}$ for $4 \mathrm{~min}$. After staining the roots were kept in glycerol until observation. $\sim 50 \mathrm{~cm}$ roots of each sample were mounted on slides and mycorrhization was quantified according to Trouvelot et al. (1986).

\section{qPCR}

iQ SYBR Green Supermix (Bio-Rad) and Bio-Rad CFX Real-Time System were used in all qPCRs. For samples from different plant species, $1 \mu \mathrm{g}$ total RNA of each sample was used for cDNA synthesis using iScript cDNA 
Synthesis kit (Bio-Rad). For samples collected by laser microdissection, pre-amplified cDNAs were used as template for qPCR. Medicago genes or fungal genes were normalized using Medicago Elongation factor 1 alpha or $R$. irregularis Elongation factor 1 alpha as reference gene, respectively. Primer sequences are summarized in Table S8. Expression values were calculated as $2^{\wedge}-\triangle$ Ct. 3 biological replicates with 3 technical replicates each were used.

\section{Subcellular localization}

LOCALIZER 1.0 (http://localizer.csiro.au/; Sperschneider et al., 2017) was used to predict nuclear localization of SPs. To experimentally verify LOCALIZER predictions, the coding sequences of SPS- without signal peptide - were cloned into an entry vector pENTR/D-TOPO or pDONR221. Primers used are summarized in Table S8. Final recombinant binary vectors were made by single-site Gateway LR reaction (Invitrogen) with GFPcontaining destination vectors pK7FWG2(N-GFP) or pK7WGF2(C-GFP) or multisite Gateway using pENTR41-35Sp::mCherry into a pKGW-RR-MGW destination vector. The fluorescently-tagged constructs were transformed into Agrobacterium tumefaciens C58. Transient agrobacterium-mediated transformation of 5-week old Nicotiana benthamiana leaves was performed by co-infiltration of GFP/mCherry-tagged constructs and the silencing suppressor $\mathrm{p} 19$. Briefly, $A$. tumefaciens strains were grown in liquid LB with appropriate antibiotics for 2 days at $28^{\circ} \mathrm{C}$. The bacteria were collected by centrifugation, resuspended in MMi medium ( $10 \mathrm{~g} / \mathrm{l}$ sucrose, $5 \mathrm{~g} / \mathrm{l}$ MS basal salts (Duchefa), $2 \mathrm{~g} / \mathrm{l}$ MES, $200 \mu \mathrm{M}$ acetosyringone, $\mathrm{pH}$ $5.6)$ to an OD600 of 0.1 and incubated for 1 hour at room temperature. Different GFP constructs together with p19 were made by mixing the appropriate bacterial suspensions in a 1:1 ratio. The suspensions were then injected into the leaves of Nicotiana benthamiana plants which were then grown a greenhouse at $21^{\circ} \mathrm{C}$. Three days post infiltration, the infiltrated parts were analysed by confocal microscopy using a Leica SP8 confocal microscope. 


\section{Results}

\section{Mycorrhization of three host plant species}

To study whether AM fungi adjust their secretome depending on the plant host species that they colonize, we analysed the fungal transcriptome in mycorrhizal roots of three evolutionary distantly related plant species. Therefore, we chose the model legume Medicago truncatula (Medicago), Nicotiana benthamiana (Nicotiana) and the monocot crop Allium schoenoprasum (Chives, which is closely related to important crops in the Alliaceae family). The three plant species were inoculated with $R$. irregularis DAOM197198 and grown under standardized low phosphate conditions $(20 \mu \mathrm{M} \mathrm{Pi}) .7$ weeks after inoculation plants were harvested

(a)
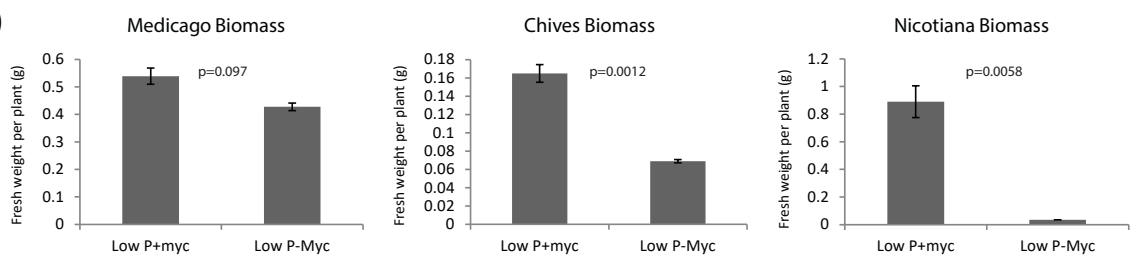

(b)

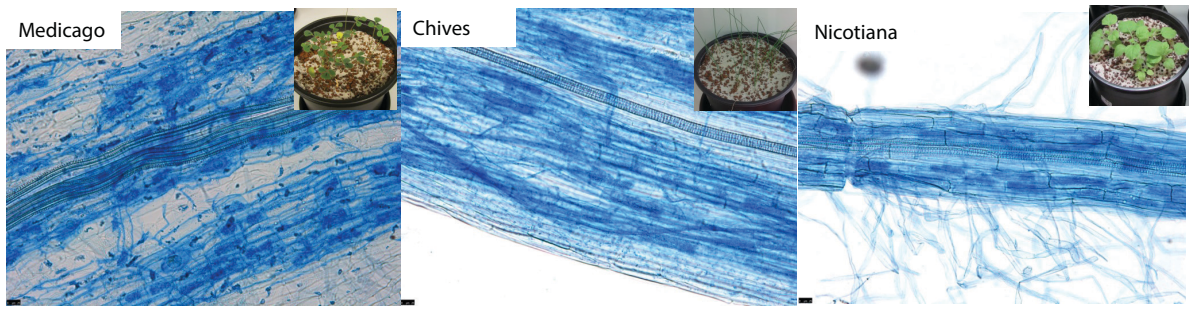

(c)

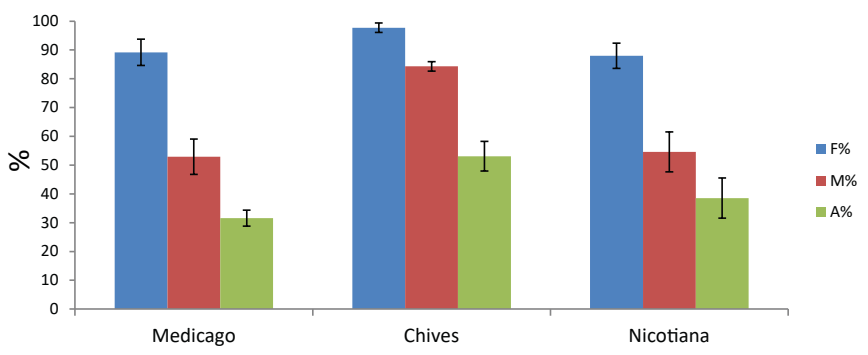

Figure 1. Mycorrhization of three host plant species Medicago truncatula (Medicago), Allium schoenoprasum (Chives) and Nicotiana benthamiana (Nicotiana). (a) Biomass of three hosts grown for 7 weeks under low $(20 \mu \mathrm{M})$ phosphate condition (-P) with (+myc) or without AM fungi (-myc). (b) Trypan blue staining of three hosts 7 weeks post inoculation. Scales $25 \mu \mathrm{m}$. (c) Mycorrhization levels are represented as frequency of mycorrhiza formation $(F \%)$, the intensity of the mycorrhizal colonization $(\mathrm{M} \%)$, and the arbuscule abundance $(\mathrm{A})$ in the root system (Trouvelot et al., 1986). 
and the mycorrhizal colonization level was determined. All three plants species were equally well mycorrhized and showed improved growth when compared to the non-mycorrhized control (Fig. 1a-c). Highest mycorrhizal growth stimulation was observed for Nicotiana. No differences in colonization strategy were observed, with all three plant species showing an Arum-type infection, forming arbuscules in the cortical cells.

\section{Defining the DAOM197198 secretome}

Several studies have predicted secreted proteins (SPs) based on the DAOM197198w genome assembly (Lin et al., 2014a), the Tisserant DAOM197198 (Gloin1) genome assembly or a combination of both (Tisserant et al., 2013; Sędzielewska Toro and Brachmann, 2016; Kim et al., 2016; Kamel et al., 2017b). As the assemblies (gene models) and prediction pipelines vary between these studies we first used our transcriptome data to verify the underlying gene models. Therefore the resulting paired-end reads were mapped to both the DAOM197198w (Lin et al., 2014a) and to the Tisserant (Gloin1) assembly (Tisserant et al., 2013). Expressed SPs were selected based on the following criteria: the presence of a signal peptide predicted by SignalP v2.0 and/or v4.1 (Nielsen and Krogh, 1998; Petersen et al., 2011), excluding SPs containing transmembrane domains (not overlapping with the signal peptide) and mitochondrial signals (according to Lin et al., 2014a). Furthermore, only putative SPs with a minimal read count of 100 total in the combined whole root samples or developmental stages were considered. This somewhat conservative cut-off allowed us to confirm the underlying gene-model and our annotation as putative SP. We noted that the transcriptome was not fully saturated based on NOIseq analyses (data not shown), which suggests that very lowly expressed genes could have been missed. In cases where the gene model in the DAOM197198w genome assembly (Lin et al., 2014a) was not supported or when a gene encoding a potential effector was missing in the DAOM197198w assembly, we adjusted the gene model or added the corresponding sequence to our genome assembly, resulting in an updated genome that was used for all subsequent RNAseq analyses (changes are summarized in Table S1; corresponding identifiers for all expressed SPs are listed in Table S2). This strategy resulted in a list of 338 genes that we consider as expressed (under our tested conditions) putative SP encoding genes (Table S2). 


\section{CHAPTER 2}

\section{Host-dependent secretome}

Next, we used the updated assembly as reference genome to perform RNAseq analyses on the three different plant species samples (Fig. S1a). Details on the mapping are summarized in Table S3a. CLC genomics workbench 10.0.1 was used to identify SPs that show significantly differential expression ( $>4$ fold-change, FDR $p<0.05$ ) between the different plant species (Fig. 2, Table S4). The vast majority (254 genes) of the SPs was more or less equally expressed in all three plant species (Fig. 2). In addition to these, 42 genes showed significant differential expression among all three host plant species (Table 1). Medicago showed the highest number of dependently induced candidate effector genes (15) compared to Chives (5) and Nicotiana (7).

1-on-1 comparison of differentially expressed genes (fold change $>4$; FDR $\mathrm{p}<0.05$ ) between two plant species, resulted in slightly higher numbers of host-dependent SP expression (Table S4). For example; 27 and 26 SPs are significantly higher expressed in Medicago compared to Chives and Nicotiana, respectively; 16 and 10 SPs are significantly enriched in Chives compared to Medicago and Nicotiana; and respectively 22 and 22

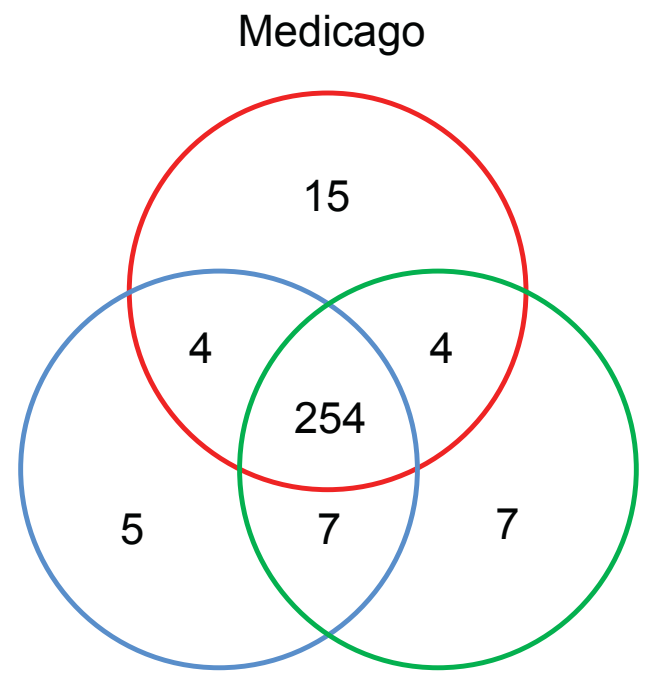

Chives

296 Nicotiana

Figure 2. Host-dependent secretome of Rhizophagus irregularis. 296 expressed $R$. irregularis SPs are grouped to their respective host plants when showing $>4$-fold higher expression levels (FDR p value $<0.05$ ) in relation to the other hosts. 254 SPs show equal ( $<4$-fold difference) expression in the three host plants. 
SPs show enriched expression in Nicotiana compared to Medicago and Chives. To validate the host-dependent expression qPCR analysis were performed on independently harvested biological samples (Fig. 3). These analyses confirmed the host-dependent expression pattern. Therefore we conclude that a subset of potential effector genes is induced in response to host-dependent cues.

Around $60 \%$ of the SPs that are expressed in a host-dependent manner (Table S2) do not have a predicted function, nor show homology to known protein motifs/domains. Three host-dependent SPs (RirG109060, RirG216030, RirG176700) contain a MD-2-related lipid-recognition (ML) domain lipid binding domain. The $M L$ domain is thought to mediate interaction with specific lipids and $M L$ domain containing proteins have been linked with regulation of lipid metabolism or innate immunity especially in animals (Inohara and Nuñez, 2002). This suggests that $R$. irregularis ML-domain effectors could play a role in lipid-related signaling between fungus and plant during distinct stages of the interaction. Additionally, the Medicago and Chives enriched SP RirG228770 shows homology to a lipase (class 3), involved in lipid metabolism/degradation. It has recently been shown that AM fungi are fatty acid auxotrophs that obtain lipids from their plant hosts (Jiang et al., 2017; Keymer et al., 2017; Bravo et al., 2017; Luginbuehl et al., 2017). Therefore it is
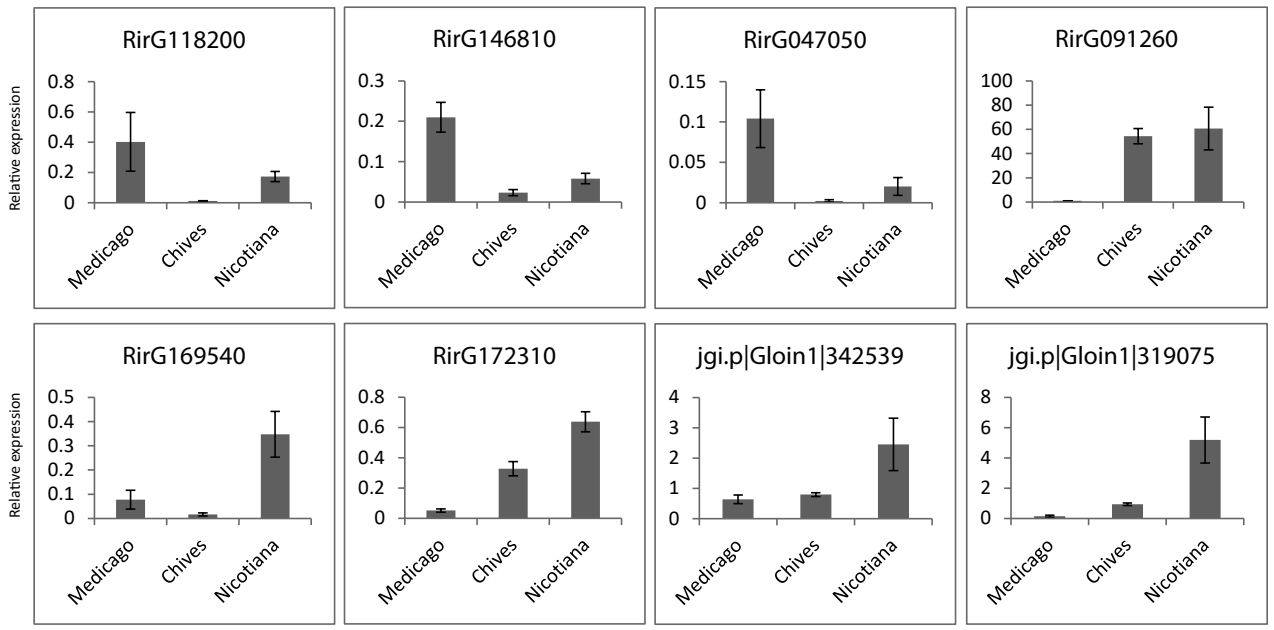

Figure 3. qPCR verification of host-dependent SPs in independent biological samples. All genes were normalized using Rhizophagus irregularis elongation factor 1 alpha (RiEF) as reference gene. Expression levels were represented as $2^{\wedge}(-\triangle \mathrm{Ct})$. Error bars indicate standard error in 3 biological replicates. 


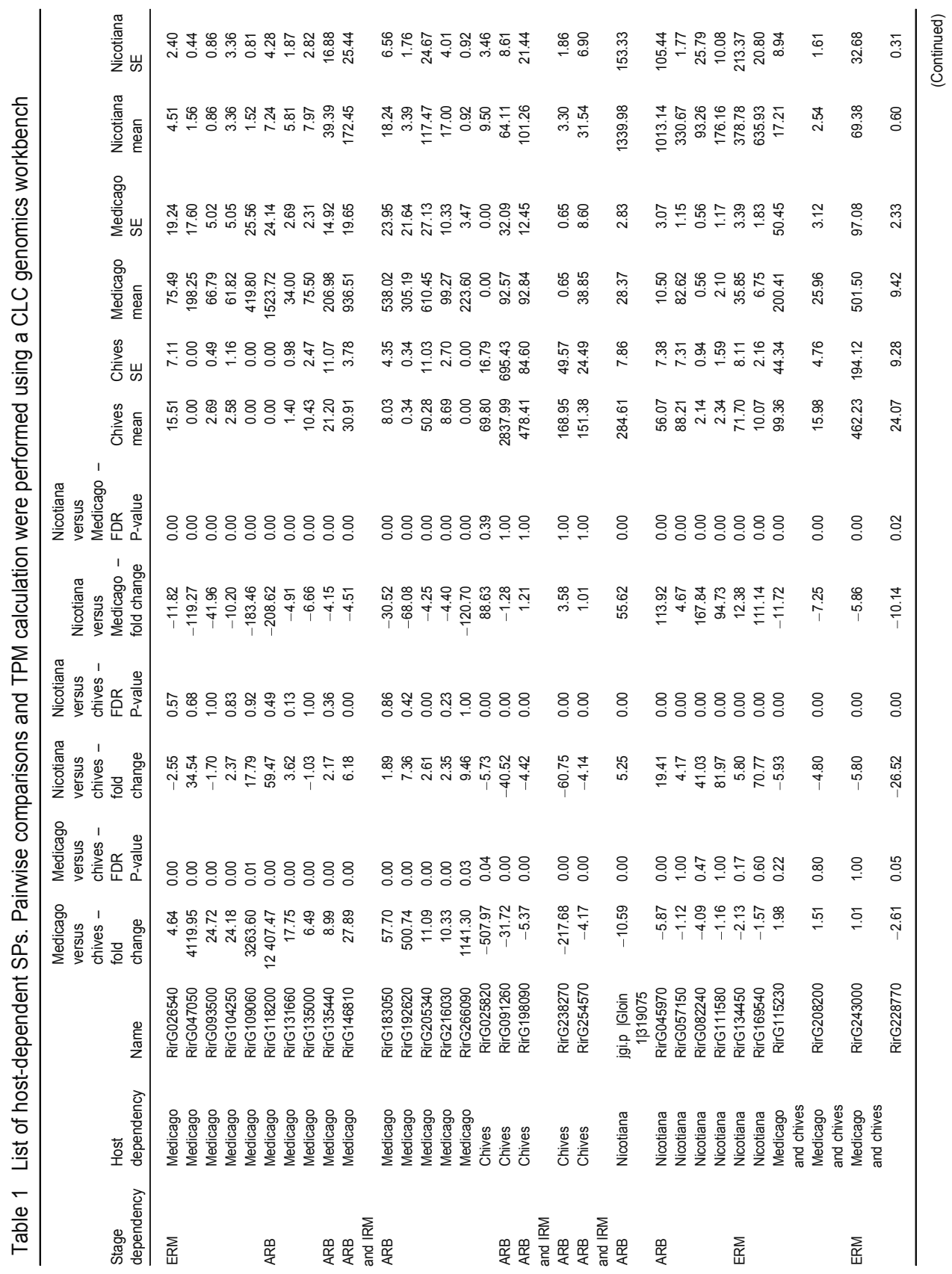




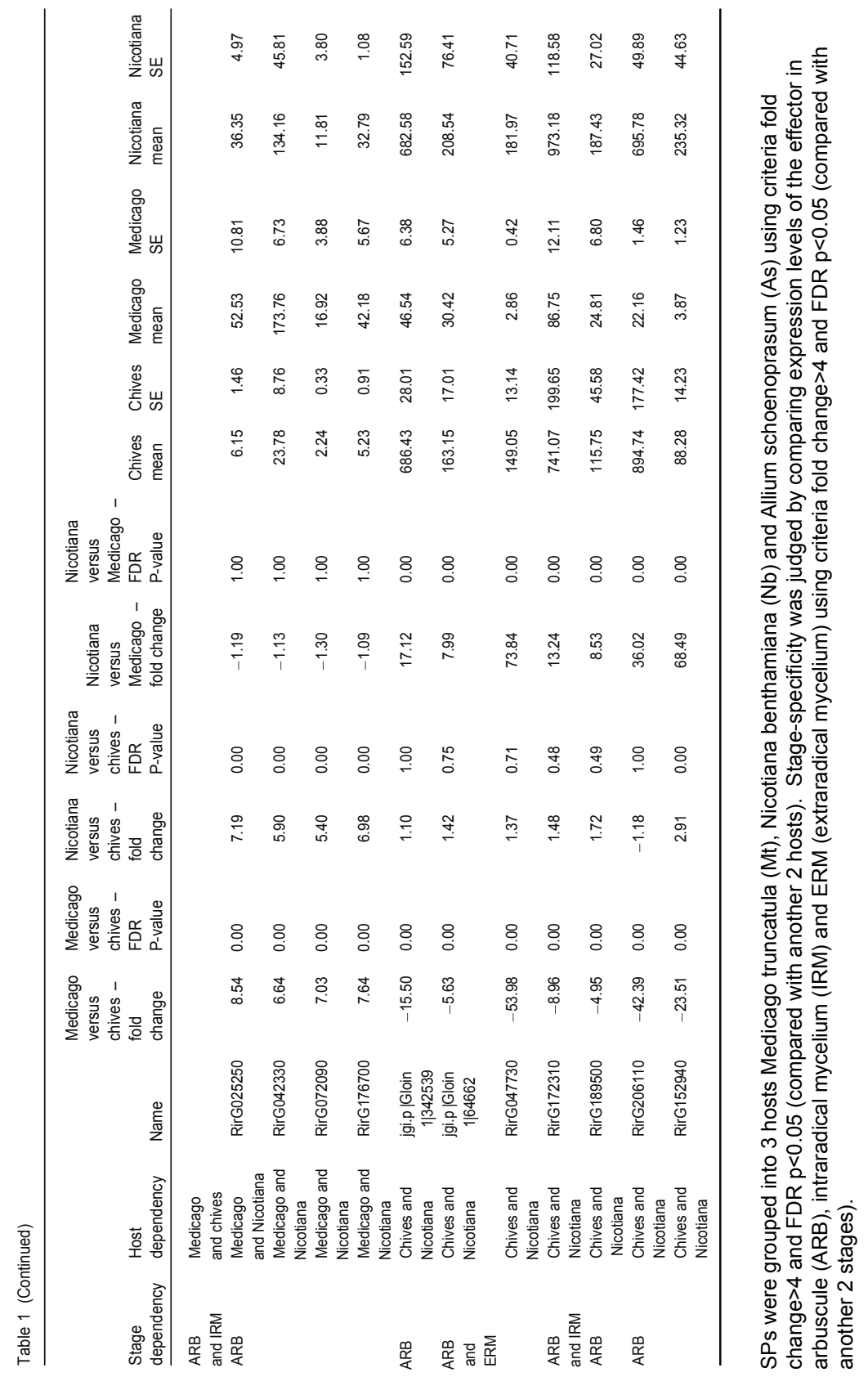




\section{CHAPTER 2}

tempting to speculate that lipid binding effectors could also play a role in lipid uptake by AM fungi, in analogy to secreted elicitin effectors from the oomycete Phytophthora that facilitate sterol lipid uptake from the plant (Mikes et al., 1998; Vauthrin et al., 1999). Six host-dependent SPs (RirG093500, RirG135000, RirG169540, RirG057150, RirG082240 and RirG134450) show homology to serine-type endopeptidases, suggesting that processing of proteins plays an important role in hostdependent interactions of AM fungi. In the interaction with Chives the SP RirG091260 is highly induced and shows homology to a mold-specific M46 protein from yeast, which has been suggested to function in drug resistance and iron and sugar transport although functional (genetic) validation is lacking (Davida Crossley dissertation, 2013; Crossley et al., 2016). Intriguingly, RirG091260 also shows homology to FGB1 from the root endophyte Piriformospora indica. FGB1 was shown to bind fungal $\beta$-glucan to suppress glucan-triggered-immunity (Wawra et al., 2016). Therefore, it will be interesting to test whether RirG091260 has a similar glucan binding activity. RirG208200 is a homolog of SP7 which was reported to modulate plant immune responses (Kloppholz et al., 2011). In contrast to SP7, which appears to be more or less equally expressed in all three host plants, RirG208200 shows significantly lower expression in the interaction with Nicotiana compared to Medicago and Chives.

Effector expression is controlled locally in response to host-dependent cues

$R$. irregularis has coenocytic hyphae were millions of nuclei migrate in a common cytoplasm (Jany and Pawlowska, 2010; Marleau et al., 2011). The hyphal network of an individual AM fungus can be in contact with multiple plant species at the same time and extend over vast area (Smith and Read, 2008). This raises the question whether AM fungi that form a connecting network between two plant species can adjust their transcriptome locally to the plant species that is colonized. To study this in relation to the host-dependent secretome, we grew Medicago and Chives in a dual compartment set-up where either plant species was grown as nurse plant inoculated by $R$. irregularis surrounded by a $45 \mu \mathrm{m}$ mesh which allows fungal hyphae to pass, but prevents plant roots from growing into the second (hyphal) compartment (Fig. 4a). This set-up allows the creation of a hyphal network that connects both plant species. After 6 weeks, Medicago seedlings were planted in the (root-free) hyphal 
compartment and grown for a further 4 weeks. Mycorrhized roots (Fig. 4b) were harvested from the inner (nurse) compartment, containing either Medicago or Chives, and from the corresponding hyphal compartment. We noted that the amount of intraradical mycelium (M\%) was reduced in Medicago roots grown in the outer compartment, although arbuscule abundance $(\mathrm{A} \%)$ did not significantly differ from the mycorrhized Medicago plants grown in the inner compartment (Fig. 4b). This suggest that Medicago was somewhat less efficient as nurse plant compared to Chives at the sampled time point. Next we analysed the expression of four selected Medicago- or Chive-dependent candidate effectors by qRTPCR (Fig. 4c).

The SPs RirG091260, RirG172310 and RirG118200 show enriched expression in either Medicago or Chives; RirG091260 is Chives-dependent and shows high expression in the Chives compartment, but not in the connected Medicago plants (Fig. 4c). RirG118200 and RirG172310 only show high expression in Medicago in line with the RNAseq data, when connected to Chives nurse plants. Therefore we conclude that an AM fungal network connected to two host plant species is able to locally respond to conditions provided by a particular host, resulting in a differential expression of SPs in different parts of the network. In addition we noticed that, in line with the RNAseq data, RirG198090 is Chives-dependent in the two compartment set-up with Chives as nurse plant, showing very little expression in Medicago (Fig. 4c). However, when Medicago was used as nurse plant, we also detected considerable expression in the older (10 week-old) Medicago nurse plants, while the younger (4 week old) Medicago plants in the second compartment hardly showed expression (Fig. 4c). Staining for mycorrhization of plants from both compartments indicated that colonization in both compartments was comparable (Fig. $4 \mathrm{~b}$ ). This suggests that the age of the plant and/or interaction creates a different local environment, which is perceived by the fungus resulting in a change in secretome.

\section{Stage-specific secretome}

To further investigate whether SPs are expressed at specific stages during the interaction with the plant, we analysed their expression in extraradical mycelium (ERM), intraradical hyphae growing in the intercellular spaces (IRM) and arbuscules (ARB) formed in Medicago root cortex cells. Laser 
(a)

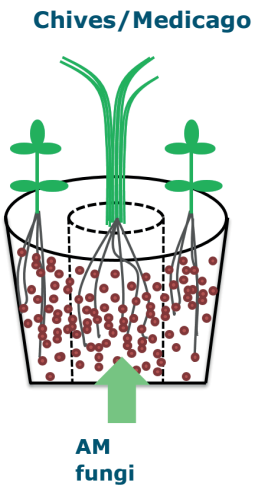

Medicago/Medicago

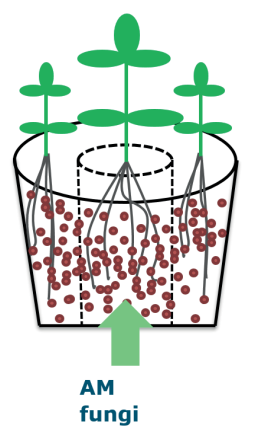

(b)
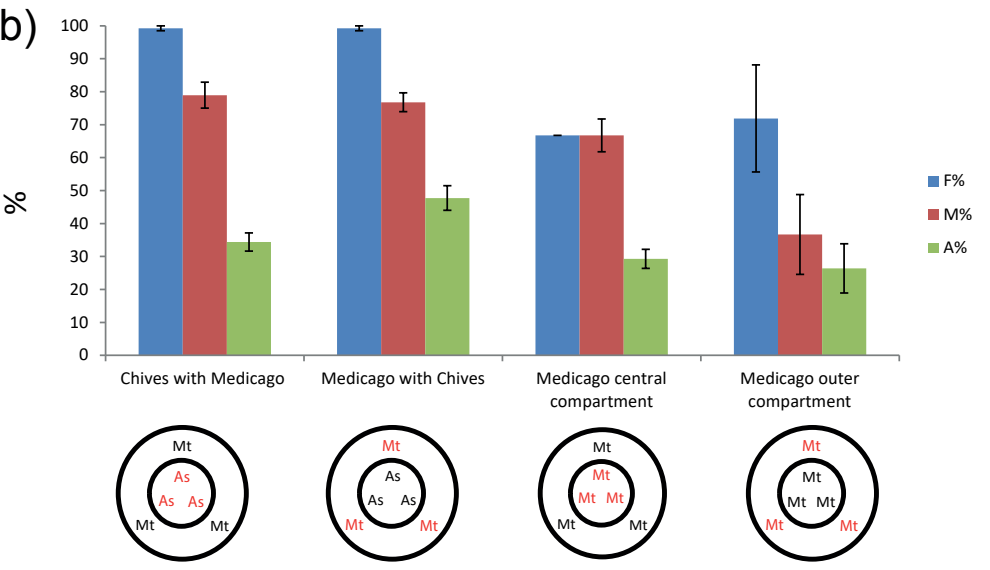

(c)

RirG1 18200

RirG172310
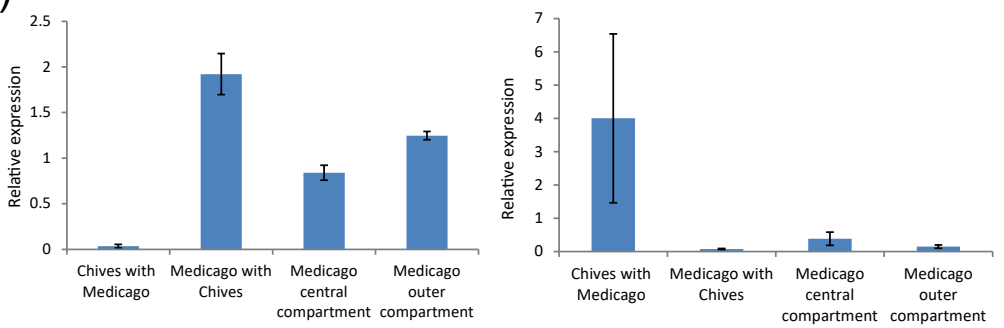

RirG091260

RirG198090
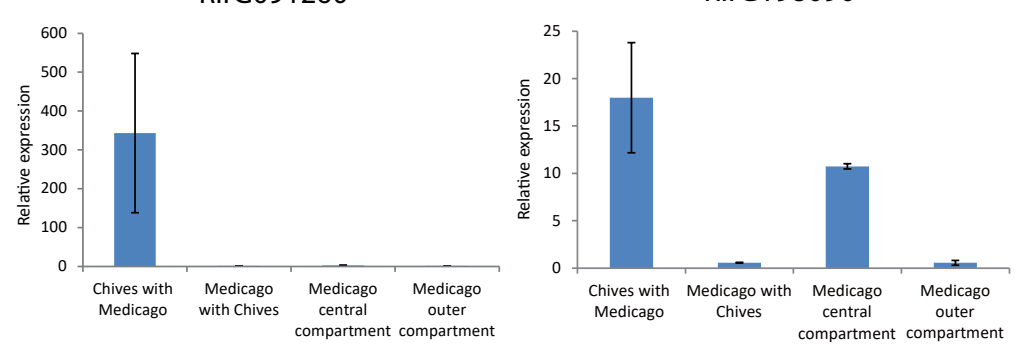
microdissection (LMD) was used to separately harvest arbuscules and cortex layers containing intercellular hyphae (Fig. 5a). Subsequently, RNA was amplified and used for library preparation and RNAseq (Table $\mathrm{S} 3 \mathrm{~b}$ and S5; Fig. S1b). In addition, we made use of the RNAseq data generated by Tsuzuki et al. (2016) representing ERM collected from axenic Medicago root cultures and germinating $R$. irregularis spores with or without treatment with the synthetic strigolactone analog GR24. Reads were mapped against the updated $R$. irregularis assembly and differentially expressed candidate effector genes were identified (Fig. $5 b, c$; Table S6a,b). The LMD-RNAseq data nicely reflect the expression pattern of studied plant marker genes known from literature (Table S7) and was verified by qPCR analysis for selected genes (Fig. S2).

119 SPs are preferentially expressed in planta compared to ERM (>4-fold enriched, FDR $p<0.05$ ). Of these, 52 SPs show specifically enriched expression in arbuscules. Only $1 \mathrm{SP}$ was specifically enriched in the intercellular hyphae (IRM), while 4 SPs show a markedly reduced expression specifically in the IRM. 66 SPs show specific/enriched expression in the extraradical mycelium (ERM) compared to ARB and IRM (Fig. 5b). 12 of the ERM-specific/enriched SPs were induced when compared to germinating spores ( $>4$ fold-change, FDR $p<0.05$; Fig. $5 c$ ). These genes are possibly induced by exudates released by plant roots. None of these genes showed induction by the strigolactone analog GR24, suggesting that other plant metabolites may be perceived by the fungus to influence their expression. All of the 5 GR24-induced SPs (RirG259300, RirG172350, jgi.p|Gloin1|348911, RirG196860 and RirG122040, Table S6) also show induced expression in ARB and IRM when compared to

continued

Figure 4. AM fungal secretome is locally regulated in response to hostdependent cues. (a) Schematic representation of the growth system. Pots were separated into a central and an outer compartment by a $45 \mu \mathrm{m}$ mesh. Only in the central compartment AM inoculum was applied. Chives or Medicago were planted into the central compartment. After 6 weeks, new Medicago seedlings were planted into the outer compartment. All plants were harvested after another 4 weeks. (b) Mycorrhization levels are represented as frequency of mycorrhiza formation $(F \%)$, the intensity of the mycorrhizal colonization (M\%), and the arbuscule abundance (A) in the root system (Trouvelot et al., 1986). (c) qPCR analysis of 4 host-dependent SPs. All genes were normalized using Rhizophagus irregularis elongation factor 1 alpha $(R i E F)$ as reference gene. Expression levels were represented as $2^{\wedge}(-\triangle \mathrm{Ct})$. Error bars indicate standard error in 3 biological replicates. 


\section{CHAPTER 2}

(a)

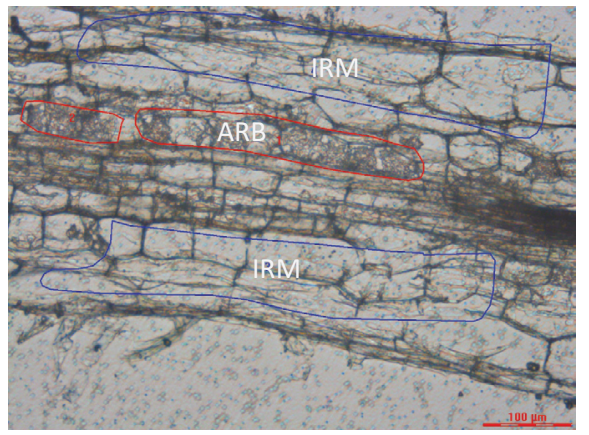

ARB

(b)

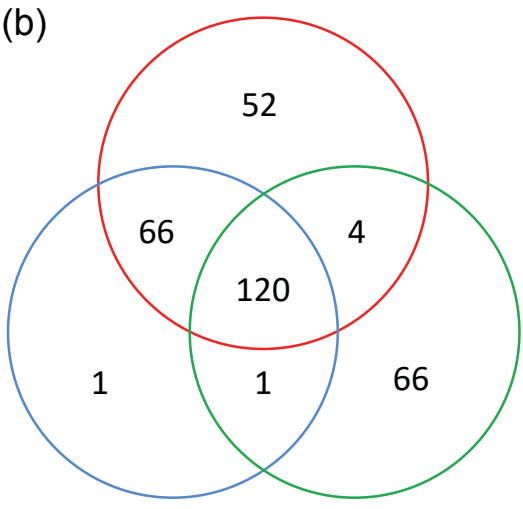

IRM

310

ERM

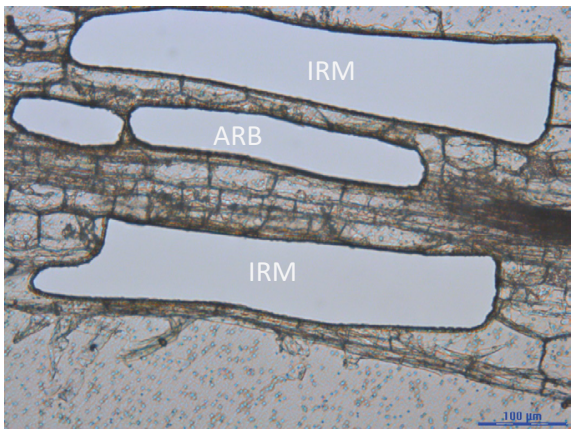

(c)

66 ERM-dependent SPs

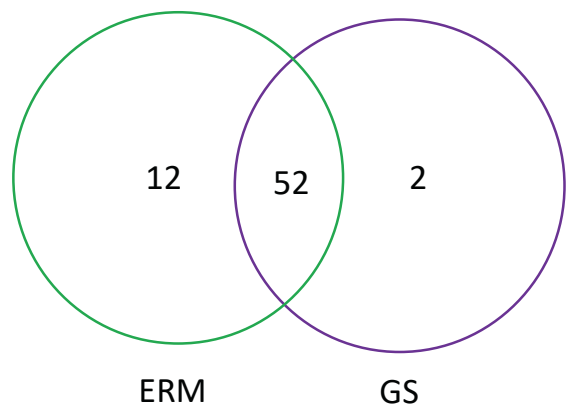

Figure 5. Laser microdissection combined with RNAseq to study stagedependent expression. (a) Arbuscules (ARB) and intraradical mycelium (IRM) were collected by laser microdissection. Scales $100 \mu \mathrm{m}$. $14 \mu \mathrm{M}$-thick section of a mycorrhized Medicago root before and after laser dissection are shown. Regions selected for microdissection are indicated. (b) Stage-dependent secretome of Rhizophagus irregularis. 310 expressed SPs were grouped into arbuscule (ARB), intraradical mycelium (IRM) or extraradical mycelium (ERM) using the following criteria: FDR $p<0.05$ and fold change $>4$. (c) 12 out of 66 SPs showing ERM-enriched expression shown in (a) are significantly higher expressed (FDR $p$ value $<0.05$, fold change $>4$ ) in ERM compared to germinating spores (GS); 2 ERM-enriched SPs show higher expression in germinating spores.

germinating spores suggesting that AM fungi perceive strigolactones also within the root tissue.

We hypothesize that several SPs that are induced in planta (ARB and IRM) are translocated to exert their function inside the host cells. Prime candidates to be translocated to host cells, are SPs that contain potential nuclear localization signals, as reported for SP7 (Kloppholz et al., 2011). 
Therefore, we screened for potential nuclear localization using the Localizer 1.0 software (Sperschneider et al., 2017) which is trained to predict the subcellular localization of effector proteins. This resulted in 86 expressed SPs with a predicted nuclear localization (Table S2). Of these, 10 are specifically expressed in arbuscules and 16 are enriched in both arbuscules and intraradical mycelium. We selected several of the predicted nuclear-targeted SPs that are highly expressed in planta, and transiently expressed fluorescently-tagged versions (either mCherry or GFP) in Nicotiana leaves. Confocal imaging of the transiently expressed tagged SPs confirms the nuclear localization of RirG128600, RirG238820, RirG245330, RirG167520. Of these RirG128600, RirG238220 and RirG167520 are targeted to the nucleolus as well as subnuclear bodies (Fig. S3). Although not confirmed by western blot analyses this is a first indication that the selected SPs may indeed be targeted to the nucleus. Four effectors that were not predicted to be nuclear, showed mostly cytoplasmic labelling (Fig. S3). However, it should be noted that translocation of nuclear targeted SPs from the fungus to the host cell remains to be demonstrated."

Of the 42 SPs that are expressed in a host-dependent manner 29 genes showed expression in the LMD data (Table 1). The majority of these (16 SPs, 55\%) show specific/enriched expression inside the root, with most (11 SPs) showing specific expression in arbuscules. 3 genes showed to be more strongly expressed in extraradical mycelium, suggesting that they likely act at the epidermis (at least in Medicago) or control fungal development outside of the root. 13 of the host-dependent SPs did not show expression in the three examined developmental stages from Medicago roots. Six of these were shown to be more prominent in Medicago compared with the other two host plants. It might be that these SPs are expressed very transiently, for example only at the onset of arbuscule formation, or at a specific developmental stage that was not included in our stage-specific analyses (such as hyphopodium formation).

Mapping of the RNAseq reads to the reference genome allowed us to rank the expression level of different SPs based on normalized read counts (TPM). Among some of the highest expressed SPs inside all three host plants, are RirG245330 and RirG172350 (ARB and IRM enriched; nuclear localization), jgi.p|Gloin1|348911 (LysM effector; ARB and IRM), RirG051530 (ARB enriched); RirG238820 (ARB enriched; nucle(ol)ar 


\section{CHAPTER 2}

localization); RirG182400 (ARB enriched; predicted nuclear), RirG167760 (ARB), RirG245610 (ARB), jgi.p|Gloin1|21945 (ARB), RirG234010 (ARB and IRM) and RirG196860 (ARB and IRM) (Table S4 and S6). Most of these SPs are specifically expressed in arbuscules, or in both arbuscules and IRM, and do not have any predicted function.

\section{Discussion}

Here we report a comprehensive and detailed analysis of the expressed SP repertoire of $R$. irregularis during the interaction with three distantly related host species and during different stages of the interaction. The vast majority ( $\sim 86 \%$ ) of the expressed SPs show equal expression levels in all three host plants, suggesting that they have conserved roles. In addition, a subset $(\sim 14 \%)$ of the SPs show differential expression depending on the host plant. This host-dependent expression is controlled locally in the hyphal network in response to host dependent cues. Such host dependent cues may be specific signals or metabolites of the plant, constitute physical properties of the root system or reflect differences in nutrient conditions that affect fungal development. These results support our hypothesis that AM SPs may act as effectors to control symbiotic efficiency in a host-dependent manner and contribute to the host preferences observed in nature.

The availability of AM transcriptome and genomic sequences has greatly facilitated the prediction of putative effectors using bioinformatics pipelines. However, different genome as well as de novo transcriptome assemblies have led to different gene models and predictions (Tisserant et al., 2013; Lin et al., 2014a). We identified a set of 338 expressed SPs that are well supported by the available RNAseq data. This set of SPs varies somewhat from the SPs/effector repertoires predicted in recent works by Sędzielewska Toro and Brachmann (2016) and Kamel et al. (2017b). Most striking was the large difference in number of predicted SPs based on the DAOM197198w assembly (Lin et al., 2014a; 550 SPs) and that of the recent paper by Kamel et al. (2017b; 872 SPs). Upon closer examination, both data sets predict only 180 SPs in common. 338 SPs predicted by Kamel (2017) and present within the DAOM197198w genome assembly were not considered as SPs in our analyses. Furthermore, 354 predicted 
SPs were not found in the DAOM197198w assembly, the majority of which were not classified as expressed SPs based on our criteria. On the other hand we predict 370 SPs that are not considered by Kamel et al. (2017b). These discrepancies are mainly caused by differences in the predicted gene models from both genome assemblies. Therefore, we only considered SPs reliable if the underlying gene models were supported by sufficient RNAseq reads. We only included expressed SPs with a minimal read count of 100 for the combined replicates. Although this may be somewhat on the conservative site, it does allow a reliable verification of the underlying gene models. For the gene models that are not covered by sufficient reads, the underlying gene model could not be convincingly verified. Such predicted SPs might be only expressed under specific conditions or at very low levels, represent pseudogenes or could be wrongfully annotated due to imperfect genome assemblies. Based on these criteria, only 10 genes predicted by Kamel et al. (2017b), lacking in the DAOM197198w assembly (Lin et al., 2014a) were considered to encode expressed SPs (Table S1). Similarly, from the 220 putative effectors predicted by Sędzielewska Toro and Brachmann (2016), we could only confirm 43 SPs supported by our RNAseq analyses. For the 78 host-induced SPs identified by Tisserant et al. (2013) we could confirm most (62) in our RNA-seq analysis. The larger overlap between our expressed SP set and Tisserant et al. (2013) is likely due to fact that both studies focus on expressed SPs in the interaction with Medicago roots. Overall, we consider our expressed SP list (Table S2) as a reliable and comprehensive set of potential effector encoding genes.

Not all secreted proteins are necessarily effector proteins. AM fungi are known for their strong ability to take up inorganic phosphate $(\mathrm{Pi})$ in $\mathrm{Pi}$ depleted soil. One important mechanism contributing to this ability is the secretion of acid phosphatases by ERM which can free phosphoryl group from organic phosphate that is otherwise not readily available to most plants (Sato et al., 2015). In line with Sato et al (2015), we found 2 SPs encoding for acid phosphatases (RirG239030 and RirG190440) that are expressed especially in the ERM. Therefore, these SPs might be of key importance for phosphate utilization in natural soils, which can consist for up to $80 \%$ of organic phosphate (Dalal, 1977).

In general fungal effectors do not share significant sequence homology to predict their function (Selin et al., 2016). Therefore, additional criteria 


\section{CHAPTER 2}

are often used to predict effectors, such as genes that are induced in planta. We identified 109 SPs that show specific induction inside the host plant root, of which 52 appear to be most specifically expressed at the arbuscule stage. These can be considered as key candidates to act as AM effectors inside the root. In support of this, most of the hostdependent SPs also appear to be specifically expressed at the arbuscule stage or inside the plant root. Although most SPs do not show significant homology to known proteins/domains, several of the host-dependent SPs suggest potential roles in lipid-related signaling or show homology to endopeptidases.

Surprisingly, we did not detect strong expression of SP7, the first characterized AM effector, in any of our tested stages compared with whole root samples. SP7 was shown to bind the pathogenesis-related transcription factor ERF19 to suppress plant immune response and to facilitate root colonization (Kloppholz et al., 2011). Previous research indicated that SP7 was prominently expressed in ERM and induced by root exudates (Kamel et al., 2017b) and increased in expression as colonization of the root increased (Kloppholz et al., 2011). Therefore, we hypothesize that $S P 7$ may only be highly expressed in hyphae that are close to the root or at the hyphopodium stage. To test this hypothesis, we collected ERM from pots containing mycorrhizaed Medicago plants, which we carefully separated into "rhizosphere" ERM close to the root and "soil" ERM relative far away from the root. QPCR analysis showed $\sim 8$ fold higher $S P 7$ expression in rhizosphere ERM compared with soil ERM (Fig. S4). This suggests that SP7 likely acts at the root epidermis. However, once the fungus has entered the plant SP7 expression is nearly switched off.

Since AM fungi have chitin and $\beta$-glucans in their cell wall, which are general elicitors of immune responses in plants, there must also be efficient mechanisms to prevent the induction of host defence when the fungus grows inter- or intracellularly inside the root (García-Garrido and Ocampo, 2002). Therefore, mechanisms different from those in the epidermis must be active to evade the immune program when the fungus is inside the root. This likely involves different effector proteins. One of such effectors may be encoded by RirG245330, which shows a domain structure (nuclear localization and repeat domains) analogous to SP7 (Lin et al., 2014a) and which is among the most highly in planta expressed SPs. Also the homology of RirG091260 to the $\beta$-glucan binding 
effector from $P$. indica (Wawra et al., 2016) may suggest a role in (hostdependent) modulation of immune responses.

We noticed that, all 5 SPs encoding chitin deacetylases (RirG030430, RirG088250, RirG159740, RirG174960, RirG242520) that are expressed in the ERM are down-regulated/switched off upon root colonization. Deacetylation of chitin, turning chitin into chitosan, has been linked to the deactivation of fungal chitin oligomers to prevent detection by the plant immune system (El Gueddari et al., 2002; Cord-Landwehr et al., 2016). Therefore, it seems counter-intuitive that $R$. irregularis down-regulates these enzymes upon colonization of the plant root. It may be related to the fact that AM fungi produce short chain chitooligosaccharides (COs) and lipochito-oligosaccharides (myc-LCOs) to activate a symbiotic signaling pathway essential for root colonization (Maillet et al., 2011; Genre et al., 2013; Gough and Cullimore, 2011). Downregulation of the deacetylases may be required to prevent the inactivation of the biological activity of symbiotic CO/LCOs (Staehelin et al., 2000). A second mechanism that pathogenic fungi use to prevent chitin from being recognized by the plant immune system is the use of LysM effectors, such as ECP6 from Cladosporium fulvum, that sequester chitin oligosaccharides released from the cell walls of invading hyphae (de Jonge et al., 2010). Interestingly, the most highly expressed SP inside the root contains a single LysM domain (jgi.p|Gloin1|348911) and is induced by the strigolactone analog GR24 (Table S6) (Tsuzuki et al., 2016). We are currently studying whether this LysM effector binds chitin and whether it can compete with immune receptors to prevent activation of an immune response or protect hyphae from plant chitinases. Since COs and LCOs also function as symbiotic signals it may even be that the LysM effector dampen or fine-tune symbiotic signaling, depending on its affinity for different chitin-like molecules.

An additional, often-used criterium to predict effector function is the presence of a nuclear localization signal, which indicates the possible translocation of effectors into the host plant cells (Sperschneider et al., 2017). Therefore, the 26 effectors that are specifically induced in planta and which we predicted to be targeted to the plant nucleus may be key candidate effectors that exert their function inside plant cells.

The next challenge will be to functionally study the role of such candidate 


\section{CHAPTER 2}

effector proteins in symbiosis. Currently there are no suitable protocols available to stably transform AM fungi, however, several studies report the use of host-induced gene silencing (HIGS) or virus-induced gene silencing (VIGS) as approaches to knock-down gene expression in the fungus (Kikuchi et al., 2016). HIGS has for example been used to silence the putative effector RirG110290, called SIS1, which we show to be most strongly expressed in the IRM (as well as arbuscules) in line with its predicted role in efficient intraradical colonization (Tsuzuki et al., 2016).

\section{Acknowledgements}

T.B. and E.L. were supported by the European Research Council (ERC2011-AdG294790).

Supplementary files listed below can be downloaded from the Plant Journal website: https://doi.org/10.1111/tpj.13908

Table S1. Updated SP-encoding gene models.

Table S2. Expressed SPs with GO annotation and LOCALIZER prediction.

Table S3. RNA-Sseq mapping details.

Table S4. Secretome of $R$. irregularis in different hosts.

Table S5. Details of LMD amplification.

Table S6. Secretome of $R$. irregularis in different stages.

Table S7. Stage-dependent markers for validation.

Table S8. Primers used in this research.

\section{Accession numbers}

The RNAseq data used in this study are submitted to the NCBI Gene Expression Omnibus, under the accession number GSE99655. 


\section{Supplementary figures}

(a)

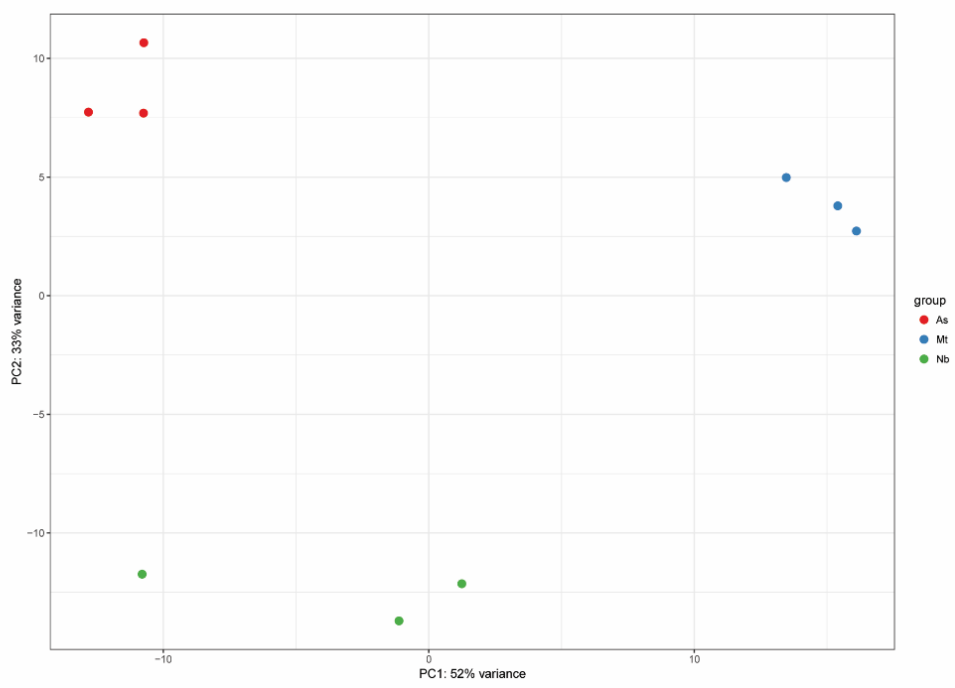

(b)

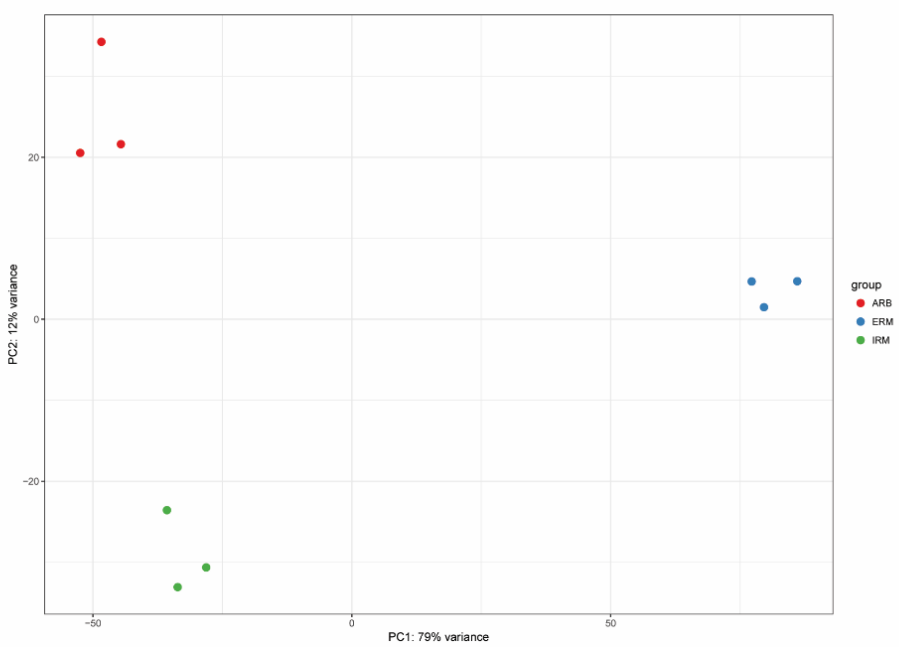

Figure S1. PCA analyses of RNASeq data. (a) PCA analysis on samples collected from different hosts Medicago truncatula (Mt), Nicotiana benthamiana ( $\mathrm{Nb}$ ) and Allium schoenoprasum (As). (b) PCA analysis on samples collected from different developmental stages of Rhizophagus irregularis. Arbuscule (ARB), intraradical mycelium (IRM) and extraradical mycelium (ERM). 
(a)

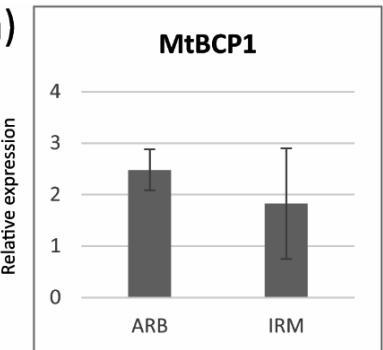

(b)
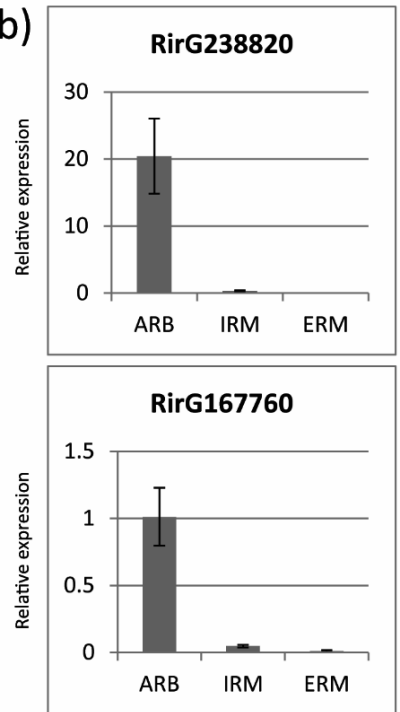
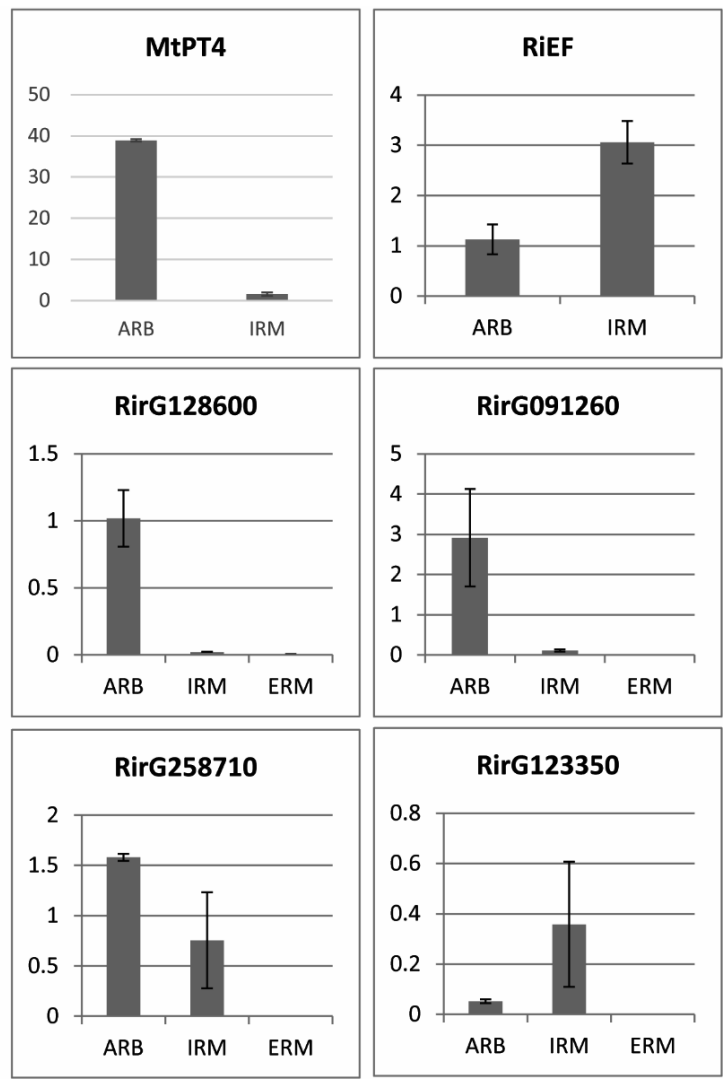

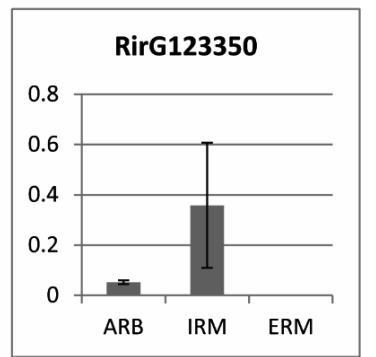

Figure S2. qPCR verification of stage-dependent expression. (a) Expression of Medicago truncatula blue copper protein 1(MtBCP1), phosphate transporter 4 (MtPT4) and Rhizophagus irregularis elongation factor 1 alpha (RiEF) normalized using Medicago truncatula elongation factor 1 alpha (MtEF1a) as reference gene. (b) Expression of selected SPs in three developmental stages. All genes were normalized using Rhizophagus irregularis elongation factor 1 alpha (RiEF). Expression values were calculated as $2^{\wedge}(-\triangle \mathrm{Ct})$. Error bars represent standard error in three biological replicates. 
(a)
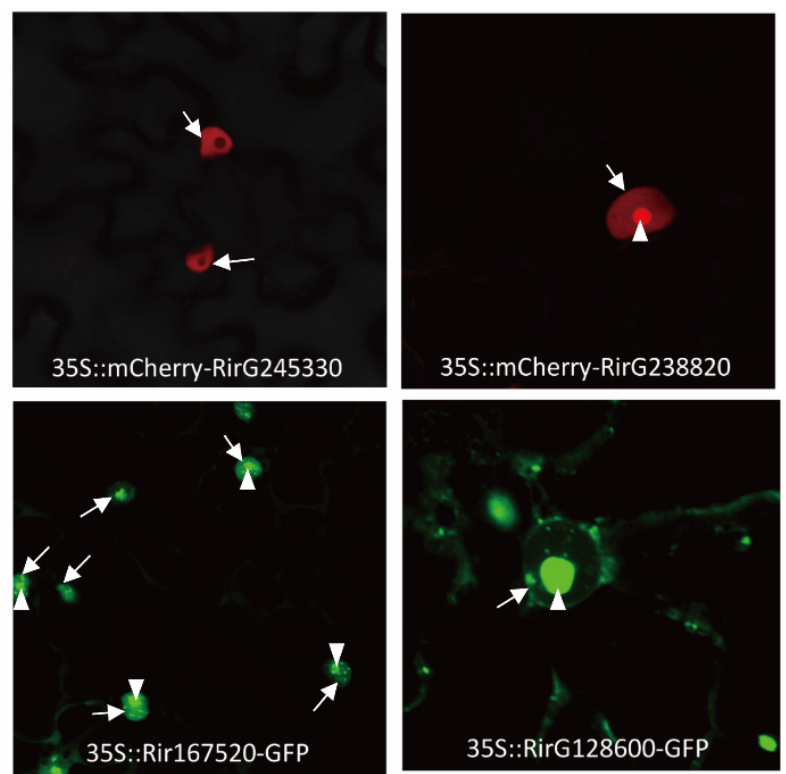

(b)
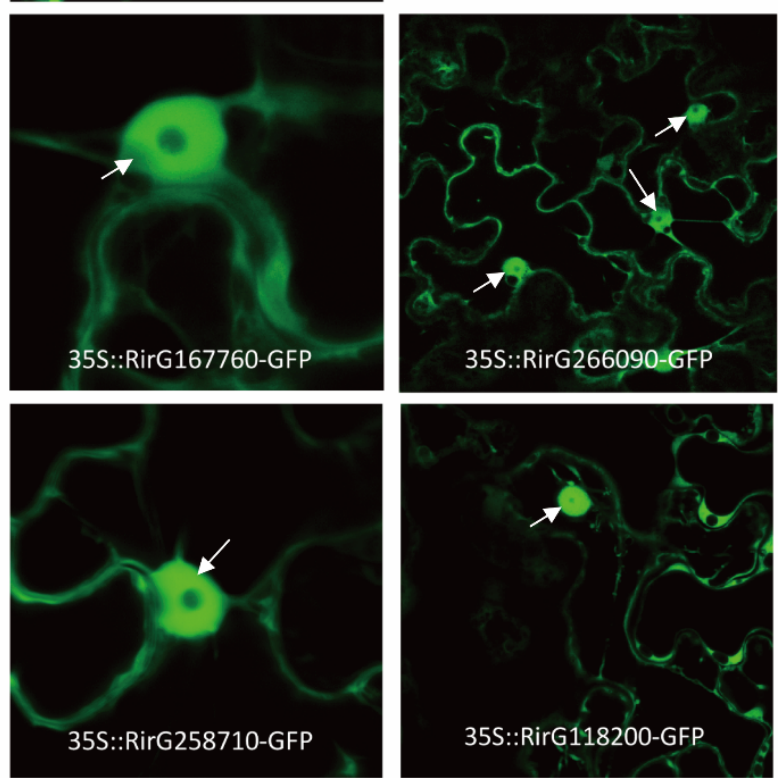

Figure S3. Validation of nuclear targeting of SP predicted by LOCALIZER software (Sperschneider et al., 2017). (a) 4 selected SPs predicted to localize to the nucleus indeed showed specific signal in the nucleus/nucleolus of Nicotiana benthamiana leaves after transient expression of fluorescently tagged fusionproteins. (b) 4 selected SPs predicted predicted to be cytosolic indeed localized to the cytoplasm. Due to the relatively small size of the SPs the fluorescent fusionproteins can also diffuse into the nucleus. Arrows indicate nuclei. Arrow heads indicate nucleolus. 


\section{CHAPTER 2}

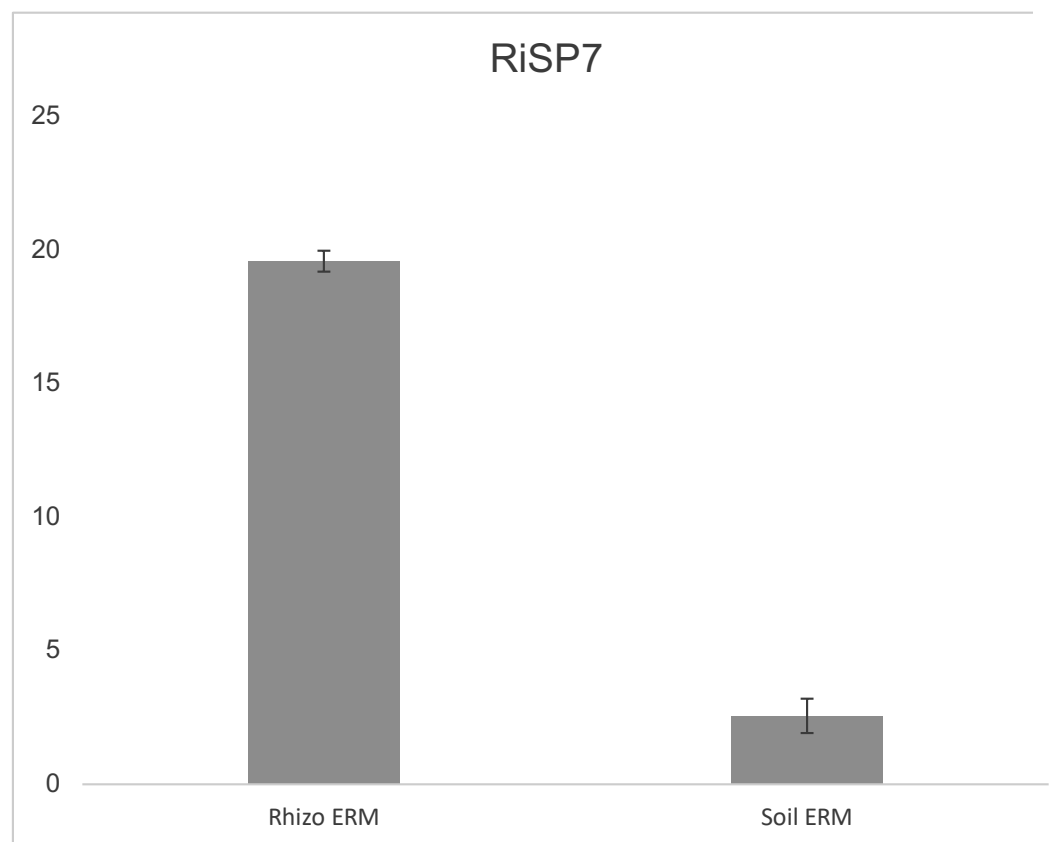

Figure S4. SP7 expression is higher in rhizosphere extraradical mycelium (Rhizo ERM) than soil extraradical mycelium (Soil ERM). SP7 expression were normalized using Rhizophagus irregularis elongation factor 1 alpha (RiEF). Expression values were calculated as $2^{\wedge}(-\triangle \mathrm{Ct})$. Four plants from 2 pots were used as 1 biological replicate. Error bars represent standard error from 3 biological replicates. 


\section{CHAPTER 3}

\section{Host- and stage-dependent insights into the transcriptome of Rhizophagus irregularis DAOM197198}

Tian Zeng, Ton Bisseling, Erik Limpens

Laboratory of Molecular Biology, Department of Plant Sciences, Wageningen University \& Research, Droevendaalsesteeg 1, Wageningen, 6708 PB, the Netherlands

* Corresponding author: Erik Limpens, erik.limpens@wur.nl

Manuscript in preparation 


\section{CHAPTER 3}

\section{Abstract}

Arbuscular mycorrhizal (AM) fungi can establish a symbiosis with the vast majority of land plants. So far, molecular research into this interaction has mostly focused on the identification of (conserved) plant components required to establish a successful symbiosis. However, how AM fungi respond and adapt to different hosts is still largely unknown. The recent availability of a fungal genome sequence together with high genomewide transcriptome analyses has started to provide first molecular insight into the fungal aspects of this key symbiosis. In Chapter 2 we focused on the secretome of the AM fungus Rhizophagus irregularis in three evolutionary distantly-related host plants and at different stages of the interaction. This showed that the fungus can adjust its secretome in a host- and stage-dependent manner. To get more genome-wide insight into fungal gene regulation, we here re-analyzed the expression profiles and performed Gene Ontology enrichment analyses to identify biological processes or functions related to host identity and stage. This identified a core set of 846 fungal genes, commonly induced ( $>2$ fold) by three different hosts compared to germinating spores, showing an enrichment in genes encoding proteins involved in transmembrane transport, oxidationreduction and tetrapyrrole binding and transferase activities. We discuss the role of several of these proteins and especially highlight the role of predicted transporters at different stages of the interaction. In addition to the commonly expressed genes, 412 genes were expressed in a hostdependent manner. The potential roles of such genes are discussed based on the GO-enrichment analyses. Overall, this work highlights the hostand stage-dependent regulation of the fungal transcriptome and offers a valuable dataset for further research into the fungal aspects of this key symbiosis.

\section{Introduction}

Arbuscular mycorrhizal fungi are obligate biotrophic fungi that form a symbiotic interaction with the vast majority of all land plants (Smith and Read, 2008). The ability of AM fungi to colonize such a wide range of hosts suggests that they must have evolved efficient mechanisms to adapt to 
the local environment of different hosts. They must be able to deal with various host immune systems and adjust their metabolism in such a way that a mutual beneficial symbiosis is maintained. Although on average AM fungi have positive effects on the performance of their host plants, numerous studies have reported that the beneficial (growth) effect on the plant (referred to as symbiotic efficiency) can vary from mutualistic to parasitic depending on both plant and fungal genotypes (Smith and Read, 2008; Sanders and Croll, 2010; Koch et al., 2017; Watts-Williams et al., 2019; Mateus et al., 2019). The molecular bases of such differences are still completely unknown. In addition to insight into the plant response to various AM fungi, it also requires a better understanding of the different responses of a single AM fungus to various host plants.

In Chapter 2 we showed that AM fungi respond to different hosts by adjusting their secretome. The transcriptome of Rhizophagus irregularis DAOM197198 was studied in symbiosis with three evolutionary distantly related host species. Furthermore, we showed that they also adjust their secretome in a stage-dependent manner. Therefore, laser microdissection was used to distinguish several stages of fungal development, including arbuscule-containing cells, cortical cells associated with intraradical mycelium (growing between cells), extraradical mycelium and germinating spores.

To get a better understanding of the transcriptional response of $R$. irregularis to the different hosts and gene regulation at the different stages, we performed a broader analysis of the acquired transcriptome data described in Chapter 2 using Gene Ontology (GO) enrichment analyses. This allowed us to identify potential molecular pathways or processes related to host identity and/or stage.

We especially address the following questions:

1) What is the fungal "core transcriptome" induced by all three different host plants?

2) Which fungal genes are expressed at different stages of the symbiotic interaction in Medicago? 


\section{CHAPTER 3}

3) Which fungal transcriptome changes are host-dependent?

This analysis identified 846 genes that are commonly induced in all three hosts compared to germinating spores. We hypothesize that these genes may reflect a "core" symbiotic transcriptome response. In addition, 412 fungal genes showed a host-dependent expression pattern. The potential role of several of these genes, based on the GO-term analyses, is discussed. Our analyses highlight the regulation of specific transporters as well as various genes involved in secondary metabolism during the symbiosis.

\section{Materials and Methods}

\section{RNASeq analyses}

Sequence reads were mapped to the $R$. irregularis genome with corrections for effector gene models (Lin et al., 2014a; Zeng et al., 2018) using CLC genomics workbench 10.0.1 (Qiagen). Sequences (RirG_identifiers) are available at http://cmb.bnu.edu.cn/Rhizophagus irregularis v10/. Original sequencing data from Zeng et al., 2018 are available from NCBI Gene Expression Omnibus GSE99655, including RNASeq data from three hosts and two stages (ARB and IRM). RNASeq data from ERM and germinating spores were downloaded from DDBJ database DRA002591 (Tsuzuki et al., 2016). Length fraction and similarity fraction was set to 0.9 during mapping and only unique mapped reads were considered in the analysis. All other parameters were set as default. TPM (transcripts per million, Wagner, Kin, and Lynch 2012) and differential expression analyses were generated by CLC genomics workbench 10.0.1. To filter the data, only genes with average TPM $>10$ across all samples were considered. To calculate genes enriched in each plant species and fungal developmental stages, a cut-off of fold change $>2$ with an FDR $p<0.05$ was used. Same parameters were used for mapping to Medicago truncatula genome V4 (Tang et al., 2014).

\section{Gene ontology analyses}


Blast2Go 4.0.7 was used to generate functional annotation of all proteins. In detail, blast searches were performed using the NCBI database. InterPro Scan function in Blast2GO was used to generate domain information. Mapping and annotation were all performed with default parameters in Blast2GO (Conesa et al., 2005).

\section{GO enrichment analyses}

GO enrichment analyses were performed using BINGO plugin (Maere et al., 2005) in Cytoscape 3.7.1 with default parameters. Only expressed genes are used as reference in GO enrichment analyses. Bingo results were imported into Enrichment Map (Merico et al., 2010) for visualization. Gene functional clusters were grouped using AutoAnnotate 1.2 (Kucera et al., 2016) in Cytoscape.

\section{Results and discussion}

\section{"Core" symbiotic transcriptome}

To obtain more molecular insight into the fungal aspects of AM symbiosis in different hosts, we first searched for fungal genes that are commonly induced in the symbiosis of R. irregularis DAOM197198 with three evolutionary distantly related host plants, Medicago truncatula (Medicago), Nicotiana benthamiana (Nicotiana) and Allium schoenoprasum (Chives). We hypothesized that such genes may reflect "core genes" that are important for the symbiosis in a wide range of hosts. Therefore, we (re-)analyzed the RNA-seq data, generated in Chapter 2 (Zeng et al., 2018), containing RNA-seq data from R. irregularis DAOM197198 in symbiosis with Medicago truncatula, Nicotiana benthamiana and Allium schoenoprasum, by setting a cut-off average TPM $>10$ across all RNA-seq samples. A list of the different RNA-seq datasets used is provided in Table S1. This identified a total of 8193 fungal genes out of 27311 annotated genes that are considered as being reliably expressed.

To identify genes that are commonly induced by all three hosts we selected genes that showed more than 2-fold higher expression (FDR 


\section{CHAPTER 3}

$\mathrm{p}<0.05$ ) in symbiotic roots compared to germinating spores (GS). This identified 1887 genes that were upregulated in at least one host (Fig. 1a). Of these, 846 genes (44.8\%) were commonly induced in symbiosis with all three hosts (Table S2). Similar numbers were observed to be down-regulated in symbiotic roots compared to germinating spores (Fig. 1b). It should be noted that this comparison does not necessarily reflect significant differences between host plants, as expression level differences between different hosts are not taken into account. A better comparison of host-dependent gene expression is described later in the section "host-dependent gene expression".

To determine at which stage of the interaction the "core" fungal symbiotic genes are expressed, we next compared the $R$. irregularis transcriptome from four different stages: arbuscules (ARB), intraradical mycelium (IRM) and extraradical mycelium (ERM), in the symbiosis with Medicago. We first selected fungal genes that were at least two fold induced in either of the three different stages in Medicago compared to germinating spores. 2883 genes were upregulated $>2$ fold (FDR $p<0.05$ ) in at least one stage compared to GS (Fig. S1). ARB and IRM shared the most upregulated genes compared to GS (1532 genes, Fig. S1). This may in part be due to contamination of ARB samples with RNA from intercellular hyphae adjacent to arbuscule cells during the laser microdissection. Next,

(a)

Upregulatedvs GS

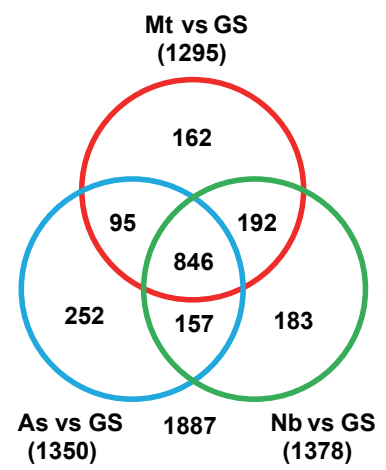

(b)

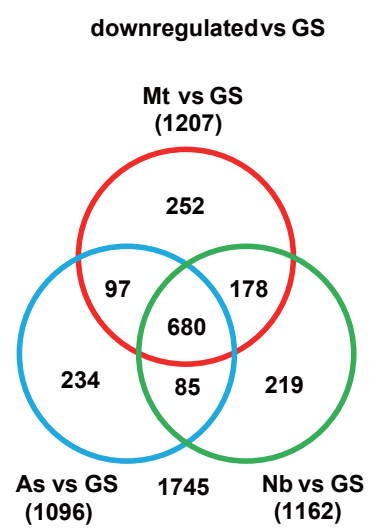

Figure 1. Genes upregulated (a) or downregulated (b) in three hosts Medicago truncatula (Mt), Nicotiana benthamiana (Nb) and Allium schoenoprasum (As) compared to germinating spores (GS). A cut-off FDR $p<0.05$ and fold-change $>2$ was used. 
we identified symbiotic stage-dependent gene expression by comparing ARB, IRM or ERM in a 1-on-1 manner with a fold-change $>2$ FDR $p<0.05$ ). 294 genes, 142 genes and 460 genes were considered as ARB, IRM or ERM-dependent, respectively (Fig. 2a). 1487 genes were classified as ARB- and IRM-dependent, whilst only 5 and 7 genes were considered as IRM- and ERM-dependent or ARB- and ERM-dependent, respectively (Fig. 2a). The relative high number of ERM-dependent genes suggests an important role for plant exudates that may affect fungal gene expression.

Among the 846 genes commonly upregulated in the symbiosis with all three hosts, 654 genes were upregulated (fold-change $>2$ FDR $p<0.05$ ) in at least one stage compared to GS. Most of those 654 genes were predominantly expressed during intraradical colonization, including 221 ARB-enriched genes, 35 IRM-enriched genes and 223 ARB- and IRMenriched genes (Fig. 2b)

\section{Gene ontology enrichment analysis of "core" symbiotic genes}

We hypothesized that genes commonly induced in all three evolutionary divergent hosts reflect "core genes" that are important for the symbiosis. To investigate the potential biological role of these protein-coding genes, we performed GO-term enrichment analyses on the 846 "core" genes (Table S2) using the BINGO plugin (Maere et al., 2005) in Cytoscape

2883 Genes upregulated

(a) in at least one symbiotic stages compared to GS

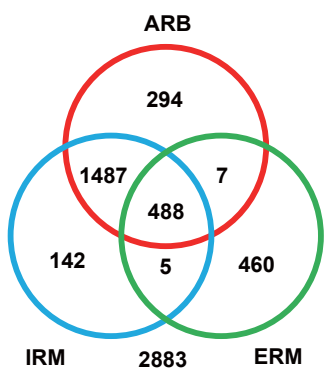

(b)

$$
\begin{aligned}
& 654 \text { Genes upregulated } \\
& \text { in at least one hosts compared to GS }
\end{aligned}
$$

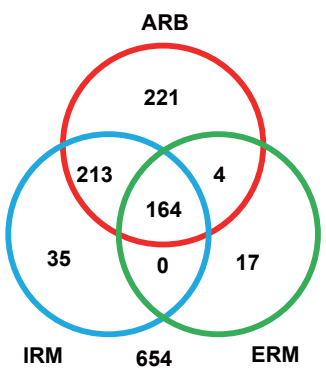

Figure 2. Stage-dependent gene expression of Rhizophagus irregularis. (a) 2883 genes upregulated in at least one biotrophic stages including arbuscule (ARB), intraradical mycelium (IRM) or extraradical mycelium (ERM) compared to germinating spores (GS) were grouped according to a cut-off FDR $p<0.05$ and fold-change $>2$. (b) Stage-dependent expression of 654 genes commonly induced in three hosts. FDR $p<0.05$ and fold-change $>2$. 


\section{CHAPTER 3}

(Shannon et al., 2003), considering GO-terms related to biological processes, molecular function or cellular localization. This revealed an enrichment in genes encoding proteins involved in transmembrane transport, oxidation reduction and tetrapyrrole binding and transferase activities (Fig. 3).

\section{Transmembrane transport}

The enrichment of genes related to transmembrane transport fits with the well-known role for AM fungi to transport mineral nutrients such as phosphate, nitrogen and other micro-nutrients to their host. In line with this, 53 core genes encoding proteins are involved in transmembrane transport (Table S2). It has been proposed that AM fungi utilize proton $(\mathrm{H}+/ \mathrm{Pi})$ - and sodium $(\mathrm{Na}+/ \mathrm{Pi})$-coupled inorganic phosphate transporters to take up Pi from the environment (Harrison and Buuren, 1995; Maldonado-Mendoza et al., 2001; Xie et al., 2016). Three of these transporters are annotated as phosphate transporters genes including two phosphate:H+ symporters (RirG093950, RirG187650) and a Phosphate/ sulfate permease (RirG046040) (Table S2). RirG187650 and RirG046040 showed ERM-enriched expression, indicating they may be involved in phosphate uptake from the environment. RirG093950 is the highest expressed phosphate transporter gene across all stages. It represents the ortholog of GigmPT, which functions as a phosphate transceptor in Gigaspora margarita (Xie et al., 2016). Consistent with previous results from Xie et al., 2016, we observed that RirG093950 is highly expressed in arbuscules as well as extraradial mycelium. Currently, it is not known how phosphate is transported from the fungus to the plant, therefore, it remains a possibility that GigmPT may function as phosphate exporter in arbuscules. Alternative candidates for phosphate export from arbuscules are SYG family (Suppressor of Yeast Gpa1) transporters (Ezawa and Saito, 2018). Interestingly, we found one yeast SYG1 ortholog RirG113890 that showed predominant expression in arbuscules. It would be intriguing to investigate whether these phosphate transporters indeed transport phosphate to the host.

In addition to phosphate, AM fungi provide nitrogen to their hosts. AM fungi can take up inorganic (ammonium or nitrate) or organic nitrogen (amino acids or peptides). The nitrogen is transformed into arginine in 
Host- and stage-dependent transcriptome

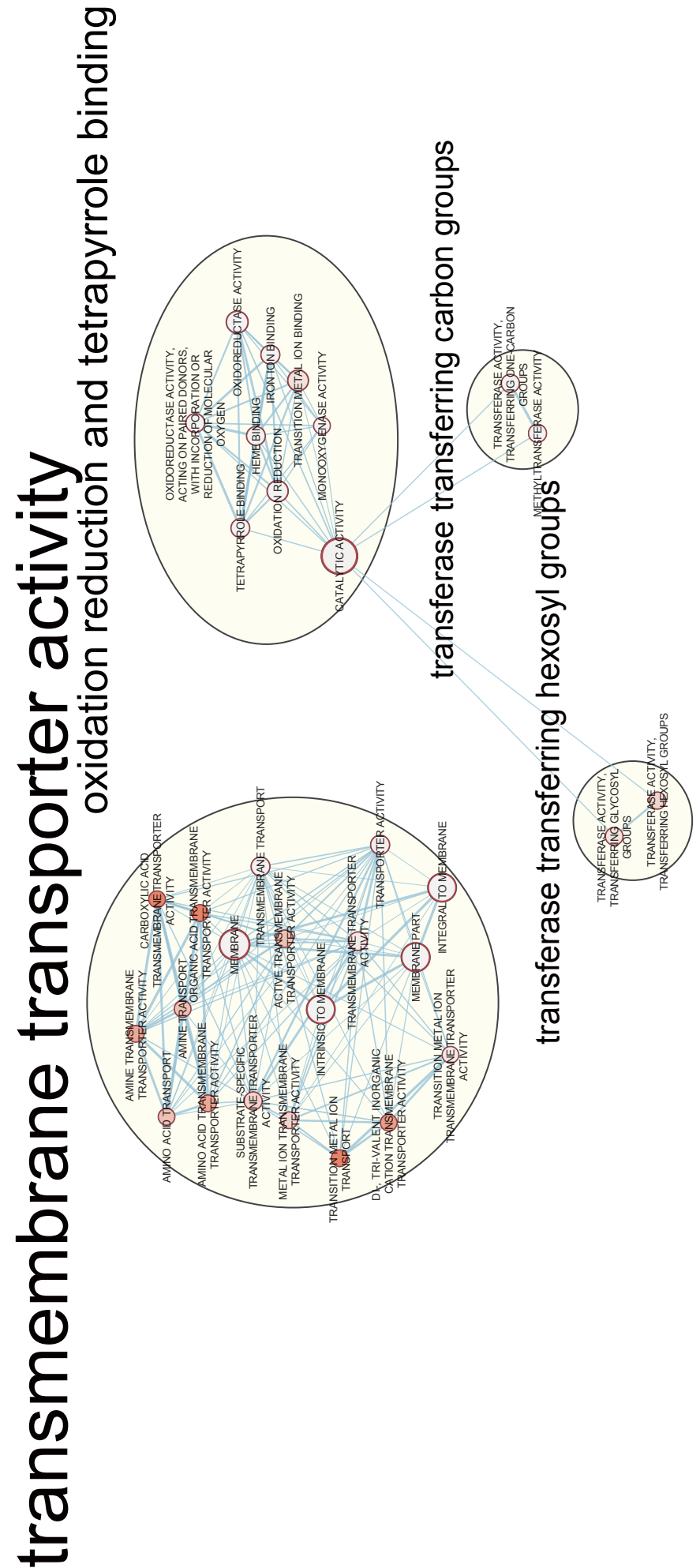

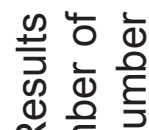

ณ 를

मे

오롣

め正

동 ญ

든

엉 인

U

흐 ब

총 움

둥

ह $\frac{\text { गे }}{\frac{\Phi}{D}}$

ह

क $\frac{1}{10}$

ฮ ฮั ฮั

ปั 등

षे

๑ ఖ

4 으 잉

은

"

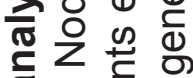

는

들 ฮั

틍 응 응

U.

든 든 당

더는 잉

친 곯

을 Ð

这

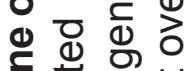

ฮั

(1) 兄

ก $\frac{1}{\Phi} \cong$

() व

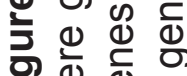

桴 \㐫 


\section{CHAPTER 3}

the ERM to be transported to the IRM via vacuoles, where it is converted back to ammonium/ammonia to be transferred to the host (Tian et al., 2010). We detected two ammonium transporters (RirG219850, RirG189010) and one nitrate transporter (RirG036130) that were induced in the symbiosis with all three hosts. The two ammonium transporters are mainly induced in ERM, suggesting that they may function to absorb ammonium from the environment. The nitrate transporter showed highest expression in arbuscules compared to any other stage, suggesting a role in providing nitrogen to the hosts, or a competition for nitrate uptake at the symbiotic interface. We also detected an enrichment of genes related to amino acid transport in all hosts. Molecular functions such as amino acid transmembrane transporter activity, organic acid transmembrane transporter activity and biological processes such as carboxylic acid transmembrane transporter activity, amino acid transport and amine transport are enriched in symbiosis with all hosts (Fig. 3). Among 5 genes related to amino acid transport, 3 genes (RirG135940, RirG097380 and RirG021210) are annotated as plasma membrane amino acid permeases, while the other two genes (RirG222330, RirG059840) are annotated as either lysosome or vacuolar membrane located amino acid transporters, respectively. It was reported that mycorrhization can induce the expression of host amino acid transporters (Guether et al., 2009a) and can affect amino acid composition of the hosts (Whiteside et al., 2012), indicating that amino acids are actively transported between both partners. The fungal dipeptide transporter RiPTR2 (RirG248040) was previously proposed to take up dipeptides in planta during the symbiosis (Belmondo et al., 2014). Consistently, we found that RiPTR2 was induced in all hosts (Table S2). However, we did not detect RiPTR2 to be induced in the intraradical stages, although it was proposed that RIPTR2 is induced in intraradical mycelium and arbuscules compared to ERM. This discrepancy might be due to different experimental conditions, or RiPTR2 may be mainly expressed in stages that were not covered in our laser microdissection, such as hyphopodia or vesicles. In contrast, we observed that most of the amino acid transporter genes (except for RirG021210) are most prominently expressed in arbuscules. Furthermore, it was proposed that AM fungi are able to take up and utilize amino acids from the environment as a source of carbon and nitrogen (Hawkins et al., 2000). Especially, peptide- and protein-bound amino acids are a source of organic nitrogen in the soil. We noticed that three putative amino acid 
transporters (RirG021210, RirG059840 and RirG232070) were expressed throughout all developmental stages. These amino acid transporters may collectively function in amino acid uptake from the environment and transfer to the hosts. On the other hand it is possible that AM fungi may acquire amino acids from their hosts as nutrients, as was proposed for biotrophic pathogenic fungi (Duplessis et al., 2011).

Since AM fungi are obligate biotrophs they fully rely on the host plant to complete their life cycle. Originally, sugars (especially hexoses) were considered to be a major carbon source from the plant to feed the fungus (Solaiman and Saito, 1997; Bago et al., 2000; Bago et al., 2003). It was shown that two Medicago sucrose synthases are upregulated during AM symbiosis, one of which (MtSUS1) was specifically upregulated in arbuscule-containing cells and required for a successful symbiosis (Baier et al., 2010). In line with a role for hexoses, a high affinity monosaccharide transporter, called RiMST2 (RirG108040), was identified in $R$. irregularis and shown to be important for proper arbuscule development (Helber et al., 2011). We identified two monosaccharide transporters (RiMST2/RirG108040 and RirG088730) that were highly induced in the symbiosis with all hosts. Both MSTs are expressed in an ARB-enriched manner, suggesting that arbuscules are a major site for monosaccharide uptake. It is currently unknown how sugars are exported from the plant cell to peri-arbuscular space. Recently, SUGARS-WILL-EVENTUALLY-BEEXPORTED-TRANSPORTER (SWEET) family members were identified as sugar efflux transporters in plants (Chen et al., 2010). Interestingly, we found a single Medicago SWEET transporter (MtSWEET1b) that was predominantly expressed in arbuscule-containing cells suggesting a function in sugar supply from the host to AM fungi in arbuscule-containing cells. In Chapter 5, we performed a detailed analyses on this SWEET transporter.

Recently, it was reported that AM fungi are fatty acid auxotrophs as they lack a key gene to synthesize fatty acids. Therefore they rely on the host plant to provide lipid precursors, such as (C16:0) $\beta$-monoacylglycerols (Luginbuehl et al., 2017; Keymer et al., 2017; Bravo et al., 2017; Jiang et al., 2017). It was proposed that such lipid precursors are transported by plant ATP-binding cassette (half ABCG) transporters (MTSTR and MtSTR2) to the fungal side (Zhang et al., 2010; Luginbuehl et al., 2017; 


\section{CHAPTER 3}

Keymer et al., 2017; Bravo et al., 2017; Jiang et al., 2017). MtSTR and MtSTR2 are specifically expressed in arbuscule-containing cells where they localize to the peri-arbuscular membrane. Knock-out mutations in these genes caused the development of stunted arbuscules (Zhang et al., 2010). However, it remains elusive how AM fungi take up lipid precursors from the host at the symbiotic interface. Seven fungal $A B C$ multidrug transporters (RirG246880， RirG181250， RirG150000， RirG134600， RirG049700, RirG010050, RirG187430) were found to be induced by all host plants (Table S2). Four of them were induced in planta, including RirG010050, RirG181250, RirG246880, RirG187430 (Table S2). Two of them (RirG181250, RirG246880) were specifically induced in arbuscules (Table S2). It is tempting to speculate that these $A B C$ transporters may mediate lipid uptake from the hosts. Alternatively, lipids can be transported by other transporter families. For instance, major facilitator family (MFS) transporters were proposed to facilitate transport of a wide range of small molecules such as oligosaccharides, amino acids as well as lipids (Madej, 2014). We found many MFS transporters (RirG261280, RirG014340, RirG019750，RirG203940，RirG204650， RirG040260， RirG088740， RirG248040，RirG181270，RirG026160，RirG234570，RirG178390， RirG129060) that are induced in the symbiosis with all three hosts. Some of them (RirG019750, RirG204650, RirG181270, RirG178390) were expressed in an ARB-enriched manner (Table S2). Recent research using electron microscopy and tomography revealed extensive extracellular vesicular structure at the plant-fungal interface (Ivanov et al., 2019; Roth et al., 2019). This raises the idea that fatty acids/lipid precursors may be transported via extracellular vesicles (Ivanov et al., 2019; Roth et al., 2019). Another intriguing possibility is the involvement of fungal effector proteins to facilitate the uptake of fatty acids. For example, elicitins, effectors secreted by Phytophthora and Pythium species, act as shuttles to take up sterols (and other lipids) from the host plants (Blein et al., 2002; Derevnina et al., 2016). Although, homologs of elicitins were not identified in $R$. irregularis, several secreted effector-like proteins contain lipid binding ML2 domains (Inohara and Nuñez, 2002). It is tempting to speculate that some of these effectors may play a similar role as elicitins to take up fatty acids from plants (Chapter 2 ).

Several transport-related "core" symbiotic genes encode for proteins that are predicted to play a role in metal ion transmembrane transport. 
For example, metal ion transporters such as iron (jgi_p_Gloin1_347887/ RiFTR1 and RirG259560/ RiFTR2, Tamayo et al., 2018), copper (RirG113850/RiCTR2, Tamayo et al., 2014), magnesium (RirG095430), nickel (RirG219210), zinc (RirG090540), calcium (RirG195010) and potassium (RirG018800, RirG095440) transporters are induced by all three hosts (Table S2). A cora-domain containing ion transporter gene (RirG022670) that may mediate Mg2+ or Co2+ transport, and a Co/Zn/ Cd efflux protein-coding gene (RirG194860) were also induced by all three host plants (Table S2). It will be interesting to investigate whether these transporters mediate metal ion uptake from or transport to the hosts. The majority of these metal transporters were most highly expressed in arbuscules compared to any of the other stages. One of the highest expressed metal transporter genes encodes a previously reported plasma membrane iron permease, called RiFTR1 (Tamayo et al., 2018). This gene (jgi_p_Gloin1_347887) was highest induced in arbuscules (TPM $594 \pm$ SE 67) compared to IRM (TPM $21 \pm$ SE 6), ERM (15 \pm SE 2) or GS (71 \pm SE 12). Another tonoplast-located iron transporter RiFTR2 (RirG259560, Tamayo et al., 2018) was also expressed predominantly in arbuscules. These results suggest that AM fungi take up iron at the interface. Even higher gene expression was observed for the copper transporter RiCTR2 (RirG113850). RiCTR2 was proposed to be a tonoplast copper transporter which pumps copper into cytosol (Tamayo et al., 2014). In contrast to the arbuscule-enriched expression pattern of the iron transporters, RirG113850 was highly induced in both ARB (TPM $1807 \pm$ SE 158) and IRM (1087 \pm SE 72) compared to ERM (14 \pm SE 5) and GS (TPM $54 \pm$ SE 4), suggesting that copper is mobilized from vacuoles to the cytosol in arbuscules and intraradical mycelium. A zinc transporter gene (RirG090540) was expressed in a similar pattern as RiCTR2, although at lower levels (Table S2). In addition, a potassium transporter gene (RirG018800) and a magnesium transporter gene (RirG095430) also showed ARB-enriched expression compared to other stages (Table S2). Taken together, these results indicate that regulation of metal homeostasis in the fungus plays an important role during the intraradical stages of the interaction. This may also be related to the ability of AM fungi to alleviate (heavy) metal toxicity in their hosts (Tamayo et al., 2014).

Oxidation reduction and tetrapyrrole binding 


\section{CHAPTER 3}

In addition to the transmembrane transport related genes, GO-term enrichment analysis identified genes encoding proteins related to oxidation and reduction, tetrapyrrole binding and transferase activities to be enriched in the "core" symbiotic fungal transcriptome (Fig. 3).

Genes related to the GO-terms oxidation and reduction that are enriched are involved in secondary metabolism; examples are cytochrome P450s (38 out of 99 genes, Table S2). Fungal secondary metabolites may function as signal molecules, virulence factors or cellular detoxifying reagents (Macheleidt et al., 2016). It was reported that some secondary metabolic pathways can be induced by local environmental factors such as low $\mathrm{pH}$ or reactive oxygen species (ROS) (Macheleidt et al., 2016). Therefore, we speculate that core genes related to secondary metabolism might be essential to detoxify ROS, to adapt to the local acidic environment surrounding arbuscules, or as virulence factors/signal molecules to colonize the hosts. In addition to the cytochrome P450s, we noticed that GO-terms related to iron binding, heme binding or transition metal ion binding were enriched in oxidation and reduction related genes. Many of these genes showed enriched expression especially in the arbuscules. Among the 100 core genes related to oxidation and reduction we observed 40 genes encoding proteins with presumed iron binding activity and 8 genes with copper binding activity. In line with the upregulation of metal transporters described above, this indicates an important role for metal ions, especially copper and iron, during the symbiosis (Tamayo et al., 2014). Among the genes encoding for copper-binding proteins, we observed six multicopper oxidases (RirG173440, RirG133820 and four Fet5p: RirG134990, RirG228480, RirG065700, RirG147730), a copper zinc superoxide dismutase (Cu/Zn SOD, RirG253800) and one copper-transporting ATPase (RirG225360). The $\mathrm{Cu} / \mathrm{Zn}$ SOD gene is highly expressed in ARB and IRM, suggesting that metals such as copper and zinc may required to detoxify ROS in ARB and IRM. Multicopper oxidases such as Fet $3 p / F e t 5 p$ in yeast are required to couple with iron transporters to facilitate iron transport (Tamayo et al., 2014). We noticed that almost all multicopper oxidases, except for RirG147730, were induced in ARB and/or IRM (Table S2). Therefore, it is reasonable to speculate that some of them may function in iron transport by coupling with RiFTR1 or RiFTR2. Iron is a metal ion has two oxidative forms, $\mathrm{Fe}^{3+}$ (ferric) or $\mathrm{Fe}^{2+}$ (ferrous), which are widely used as electron donor/acceptor in biochemical 
reactions such as lipid metabolism, TCA cycles, glycolysis and secondary metabolism. Interestingly, perturbation of iron homeostasis plays also a key role during host-pathogen interactions (Expert et al., 1996; Verbon et al., 2017). For example, it has been reported that a high affinity iron permease expressed in intraradical hyphae of the Ustilago maydis is essential for pathogenicity of this biotrophic smut fungus (Eichhorn et al., 2006). Further, the plant immune system was shown to target the iron acquisition system in Pseudomonas syringae to inhibit bacterial growth (Nobori et al., 2018). Interestingly, we noticed that all expressed Medicago IRT genes, which encode for iron transporters (Vert et al., 2002), were specifically downregulated in arbuscule-containing cells (Fig. 4a) These data suggest that AM fungi may take up iron from the peri-arbuscular space. It is reported that AM fungi can also facilitate iron uptake by their hosts. However, this may be host and/or condition dependent as it was shown that AM symbiosis enhanced iron status in sorgum but not in peanut (Caris et al., 1998). Whether AM fungi provide iron to or take up iron from the host is probably due to the local iron status of AM fungi. Iron is considered to be a crucial mineral nutrient for fungal growth, while at the same time playing an essential role in plant immunity because of its indispensable role to generate ROS. Therefore, iron is considered to be tightly co-regulated during plant immune responses, either to withhold iron from the microbes or to generate extracellular ROS (Verbon et al., 2017). This raises the question whether uptake of iron from the peri-arbuscular space is also essential to subvert host immunity. In the model plant Arabidopsis thaliana, a $\beta$-Glucosidase gene BGLU42

(a)

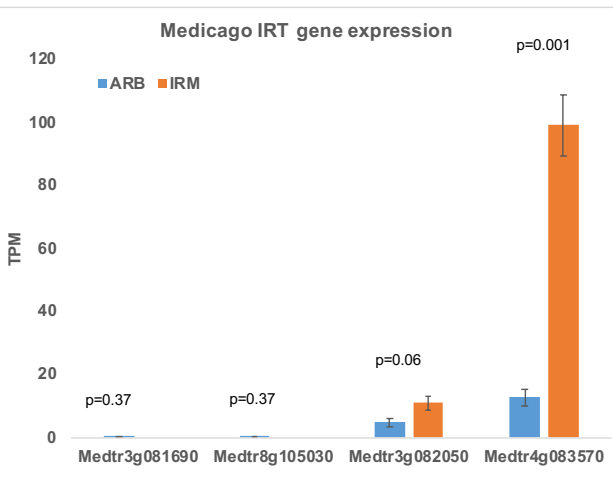

(b)

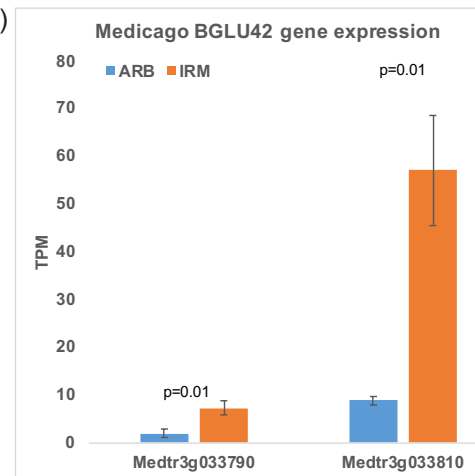

Figure 4. Iron-related genes expression (TPM) in Medicago in arbuscules (ARB) or intraradical mycelium (IRM). Error bars represent standard error of three biological replicates. Student t-test $p$-values were marked. 


\section{CHAPTER 3}

controls the secretion of the iron-chelating phenolic compound coumarin under iron deficiency (Zamioudis et al., 2014). BGLU42 converts the coumarin scopolin to scopoletin, which can be secreted to the apoplast or rhizosphere (Stringlis et al., 2018). Secreted scopoletin on one hand can chelate $\mathrm{Fe}^{3+}$ to bring it to the membrane-bound ferric reductase to be reduced for uptake (Stringlis et al., 2018). Additionally, it can directly inhibit the activity of fungal pathogens such as Fusarium oxysporum and Verticillium dahliae (Stringlis et al., 2018). Interestingly, we noticed that the Medicago orthologs (Medtr3g033790, Medtr3g033810) of AtBGLU42 are downregulated in arbuscule-containing cells compared to cells surrounding intercellular hyphae (Fig. 4b). Therefore, downregulation of host BGLU42 in arbuscule-containing cells might be crucial for the fungus to absorb iron from the peri-arbuscular space while avoiding the toxification of the fungus. Taken together, these results suggest that plants and AM fungi may have evolved a cooperative mechanism for iron transport to feed the fungi, while avoiding the induction of iron-dependent plant immune responses.

\section{Transferase activity}

We also observed enriched molecular functions related to transferase activities, such as methyltransferase activity and glycosyltransferase activity (Fig. 3). Among the 29 genes predicted to convey methyltransferase activity, 3 were annotated as DNA methyltransferases (RirG033390, RirG058940, RirG269690). Two of them (RirG058940 and RirG269690) were induced in ARB and/or IRM (Table S2), suggesting that epigenetic changes, such as DNA methylation, are triggered during the colonization of plants. It has been reported that $A M$ inoculation enhances host DNA methylation (Varga and Soulsbury, 2018). However due to the limitation of the method used, this study was unable to distinguish between DNA methylation in the plant or fungus. Therefore, part of the enhanced DNA methylation might have occurred in the fungus. In addition, two methyltransferases (RirG228170 and RirG172140, Table S2) involved in fatty acid metabolism, which are annotated as sterol 24-C methyltransferase and cyclopropane-fatty-acyl-phospholipid synthase respectively, were induced in all hosts, supporting an active lipid metabolism during upon symbiosis. Among them, the cyclopropanefatty-acyl-phospholipid synthase (RirG172140) is expressed especially in 
the arbuscules. Among the induced genes encoding glycosyltransferases, we detected several chitin synthase genes (RirG064340, RirG085340, RirG173030, RirG266310, Table S2). All chitin synthase genes showed highest expression in arbuscules compared to the other stages, suggesting that cell wall remodeling is most active in arbuscules. In addition, adenine-(RirG231270), uracil-(RirG128640) and amido(RirG034140) phosphoribosyltransferases were found to be induced by all three hosts (Table S2). Except for the amido-phosphoribosyltransferases (RirG034140), both adenine-(RirG231270) and uracil-( RirG128640) phosphoribosyltransferases were strongly active in ARB and/or IRM (Table S2), pointing to increased nucleoside metabolism in the intraradical stages. This could be related to a higher energy demand and active fungal growth at these stages.

\section{Host-dependent transcriptome}

In addition to the induced core genes shared by the different hosts, we next examined host-dependent gene expression profiles. Therefore, we performed 1-on-1 comparisons on the transcriptome data from the three hosts to look for genes that are significantly enriched (Fold-change $>2$; FDR $p<0.05)$ in each host. This revealed 224 host-dependent genes (including 7 putative effectors) that show enriched expression in Chives, 104 genes (including 22 putative effectors) enriched in Medicago and 84 (including 25 putative effectors) in Nicotiana (Fig. 5a). The two dicot hosts showed more overlap (172 genes, including 23 putative effectors) compared to Nicotiana/Chives (157, including 15 putative effectors) and Medicago/Chives (116, including 4 putative effectors) (Fig. 5a). 90\% of expressed genes are commonly expressed in all host (7336/8193). In total, 857 out of 8193 expressed genes showed host-dependent expression. Genes encoding for putative effectors showed relatively more host-dependent regulation compared to non-secreted proteins (96 out of 292), suggesting that effectors may play an important role in hostdependent colonization. However, this does not seem to be the rule for all hosts, as only a very low fraction of effectors were enriched in chives compared to the other two hosts.

To get a clue on the molecular role of the host-dependent genes we again used the Bingo plugin in Cytoscape for a GO-enrichment analysis. 
(a)

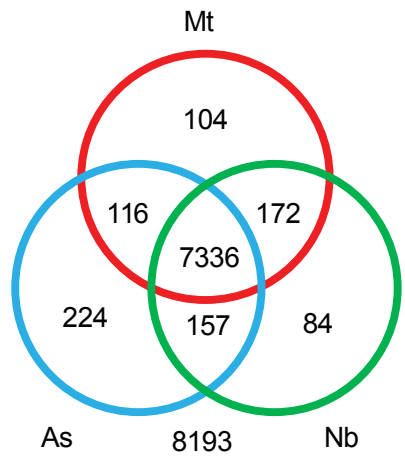

(b)

\section{oxidoreductase activity}

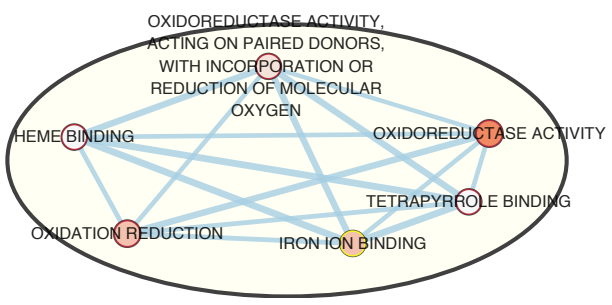

(c)

\section{serine hydrolase activity}

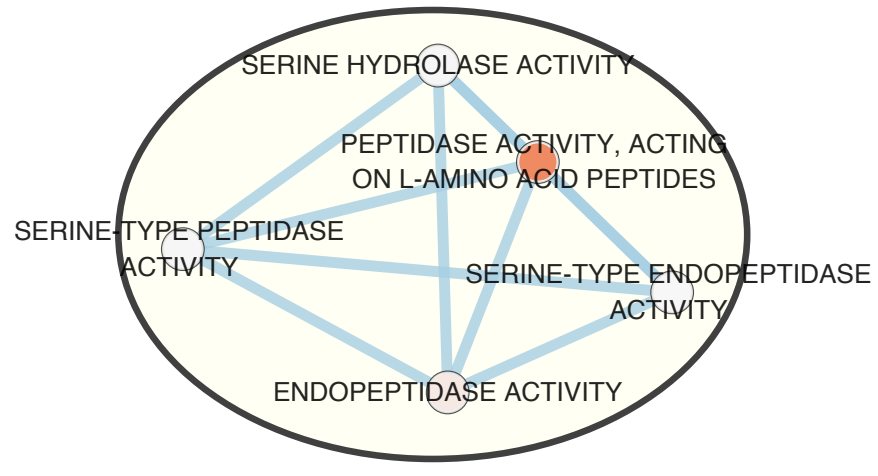

(d)

\section{amino acid metabolic processes}

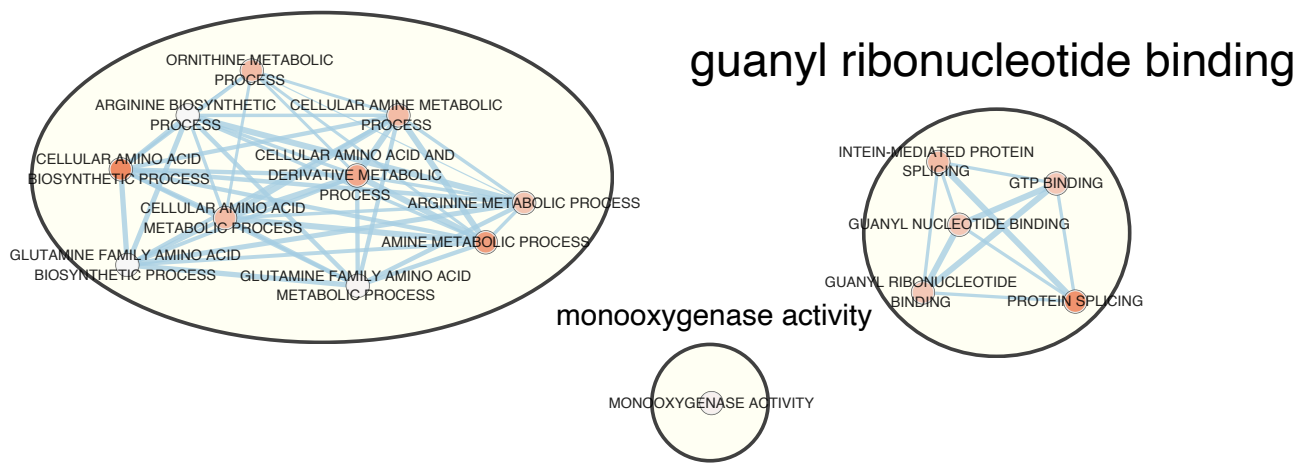


(e)

(f)

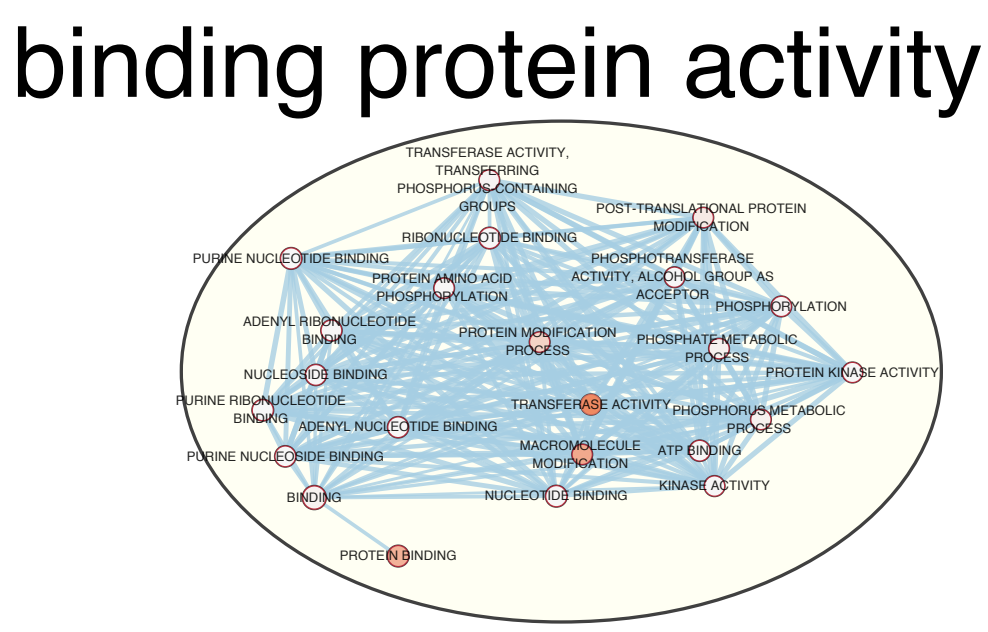

(g)

\section{binding protein activity}

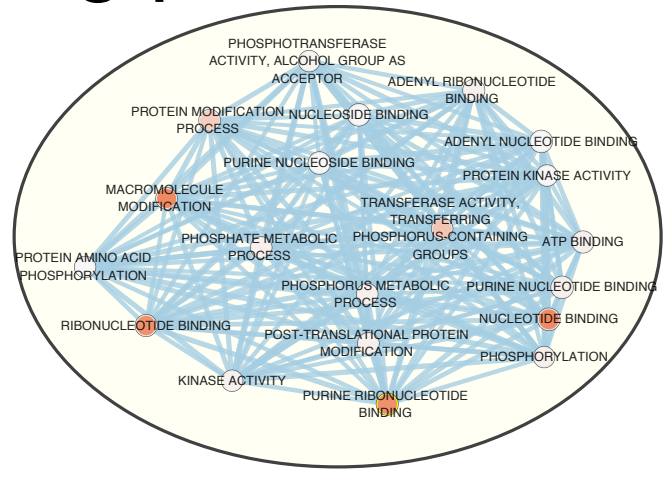

glutathione biosynthetic process

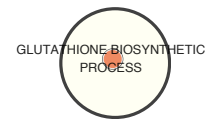

Figure 5. Host-dependent gene expression of Rhizophagus irregularis. (a) 8193 expressed genes were grouped according to FDR-p $<0.05$ and fold-change $>2$. Host-dependent genes were then used for gene ontology (GO) enrichment analyses. GO enrichment results are shown in b (Chives), c (Nicotiana), d (Medicago), e (Medicago and Nicotiana), $\mathrm{f}$ (Medicago and Chives) and g (Nicotiana and Chives). Results were generated using Enrichmentmap. Nodes represent gene sets. Node size represents the number of genes in the gene set. Node fill represents enrichment score (q-value). Edge width represents the number of genes that overlap between a pair of gene sets. 


\section{CHAPTER 3 \\ Chives-enriched genes}

Among the genes showing higher expression in Chives compared to the other two hosts, genes associated with the GO-term oxidoreductase activity were enriched. We especially observed genes enriched related to heme binding, tetrapyrrole binding, iron binding and oxidoreductase activity (Fig. 5b). Of the 26 genes showing oxidoreductase activity, 11 (RirG262020，RirG134710， RirG037340， RirG180500， RirG075590， RirG191460，RirG133840，RirG208770， RirG259940， RirG134680， RirG134670) encode cytochrome P450 (Table S3). Most of those genes were expressed highest in ERM or in germinating spores. However, two genes (RirG262020, RirG134680) were expressed in an ARBdependent manner, suggesting that they may be involved in arbusculedependent secondary metabolism. Among the genes with annotation, a gene annotated as beta-lg-H3 fasciclin (RirG182600) was found (Table S3). Fasciclins are membrane bound glycoproteins that are present in all kingdoms of living organisms (Seifert, 2018) and are considered to function in cell adhesion (Kim et al., 2000). A fungal fasciclin-like protein has been reported to be involved in pathogenicity of the biotrophic rice blast fungus Magnaporthe oryzae (Liu et al., 2009). A recent proteome research also detected two fungal fasciclin-domain containing secreted proteins in bean leaves infected by the biotrophic rust fungus Uromyces appendiculatus (Cooper et al., 2016). Fasciclin-domain containing proteins are aslo very prevalent in symbiotic intractions, as they have been discovered in a wide range of symbioses, including Nostoclichen, rhizobium-legume, and alga-cnidarian symbioses (Paulsrud and Lindblad, 2002). Considering the general role of the fasciclin domain in cell adhesion, we speculate that the AM fasciclin plays a similar role in the contact with plant cells. Additionally, we found a fatty acid desaturase (RirG048640), a fatty acid ligase (RirG198740) and a fatty acid oxygenase (RirG030490) that were higher induced in Chives compared to Nicotiana and Medicago (Table S3), indicating that AM fungi may have hostdependent lipid metabolic processes. Whether the higher expression of these genes in Chives compared to Medicago and Nicotiana means that AM fungi receive different fatty acids from different hosts remains to be studied. Intriguingly, a recent study by Kameoka et al. (2019) suggested that different AM fungi respond differently to fatty acids in relation to sporulation activity. It would be highly interesting to test whether this may be linked to host preferences observed in nature for distinct AM fungal 
interactions. Two zinc transporter genes (RirG090540 and RirG021750) were expressed in a Chives-dependent manner (Table S3). This may indicate that AM fungi consume or mobilize more zinc when they colonize Chives. Interestingly, two enzymes involved in tRNA modification, tRNA (cytidine(32) guanosine(34)-2 -0)-methyltransferase, and tRNA uridine 5-carboxymethylaminomethyl modification enzyme were also enriched specifically in Chives (Table S3). Host-dependent expression of the tRNA modification enzymes suggests that AM fungi may regulate translation or ribosome structure in a host-dependent manner. In a recent study, a heterogeneous non-tandem rDNA structure has been observed for AM fungi (Maeda et al., 2018). A similar heterogenous rDNA structure was observed in the malaria parasite Plasmodium and this was hypothesized to alter ribosome properties, affecting translation, to facilitate host adaptation to different hosts (Maeda et al., 2018).

Nicotiana-enriched genes

GO-term analyses revealed an enrichment of Nicotiana-dependent genes related to endopeptidases activity (Fig. 5c/Table S3). Among 84 genes, there were 6 serine endopeptidase encoding genes (RirG017560, RirG169540, RirG134450, RirG082240, RirG226960, RirG057150, Table S3). These may play a role in the processing of (pre-)proteins that may be essential to colonize Nicotiana. Recent genomic and transcriptomic analyses indicate that several putative AM fungal effectors including the previously identified SP7 might be processed by a subtilisin-type serine endopeptidase KEX2 before secretion (Kamel, Tang, et al., 2017). Therefore, it will be interesting to investigate whether host-dependent proteases regulate the modification of fungal effectors. Another possibility is that the secreted peptidases target plant proteins to suppress defense responses.

Medicago-enriched genes

The 104 Medicago-dependent genes (Table S3) were enriched in genes related to amino acid metabolic processes, guanyl ribonucleotide binding activity and monoxygenase acitivity (Fig. 5 d).

Genes associated with guanyl ribonucleotide binding activity encode 


\section{CHAPTER 3}

proteins with GTP binding or GTPase activity (RirG103750, RirG112970, RirG192840, RirG192850, RirG234000, RirG251710, RirG093940). Two of these genes (RirG112970, RirG234000) are highly induced in ARB and IRM (Table S3), indicating that they might be important for intraradical colonization. Among the genes related to amino acid metabolic processes, we mainly observed genes related to ornithine, arginine or glutamine metabolism. A glutamine synthetase (RirG081420) and a gene encoding a carbamoyl-phosphate synthase small chain (RirG211460), which catalyzes the transition from glutamine to ammonia, were highly expressed in Medicago (Table S3). Generated ammonia can be used by the carbamoyl-phosphate large chain to synthesize carbamoyl phosphate, a precursor of arginine biosynthesis. Consistently, we also noticed that carbamoyl-phosphate large chain (RirG132190) and ornithine carbamoyltransferase (RirG132210) are also higher expressed in Medicago compared to the other two hosts (Table S3). Except for RirG132210, which was mainly expressed in GS, all the other genes were most highly expressed in both ERM and GS (Table S3). All three enzymes are operating in pyrimidine biosynthesis, arginine biosynthesis or the urea cycle. In addition, an argininosuccinate lyase (RirG187750) and an argininosuccinate synthetase (RirG111970) which function in arginine biosynthesis, were found among the Medicago-dependent genes (Table S3). Both of them showed strong expression in ERM (Table S3). These results suggest that nitrogen metabolism in the fungus is more active when colonizing Medicago. As a legume, Medicago has a high demand for nitrogen to sustain its high protein levels. Since arginine is thought to be the main nitrogen-storage form that is transported from the ERM to the IRM, it may be that the higher $\mathrm{N}$-demand of Medicago triggers a higher $\mathrm{N}$-supply by the fungus.

Genes with monoxygenase activity include five cytochrome P450 genes (RirG266190，RirG262310，RirG066260，RirG091350，RirG091380) that were higher expressed in Medicago compared to Chives and Nicotiana (Table S3). Four of them (RirG266190, RirG091380, RirG066260, RirG091350) showed the highest expression in arbuscules (Table S3), suggesting an important function in arbuscule development or maintenance through the formation of secondary metabolites or detoxification of host-specific metabolites. As potential candidate transcription factors that may control some of the host-dependent genes we found two AP-1 type transcription 
factors (RirG251910, RirG150060) to be expressed in a Medicagodependent manner (Table S3). Fungi, especially fungal pathogens, have evolved efficient mechanisms to detoxify ROS, for example by producing enzymes such as superoxide dismutase, catalases, peroxidases or redoxins. It was reported that an AP1-like bZIP transcription factor confers oxidative stress resistance in Sacchromyces cerevisiae by regulating thioredoxin expression (Toone et al., 2001; Kuge and Jones, 1994). Its ortholog in the rice blast fungus Magnaporthe oryzae, called MOAP1, mediates the oxidative stress resistance and is essential for pathogenicity (Guo et al., 2011). AP1-like transcription factors have also been implicated in symbiotic interactions, as an AP1 transcription factor was shown to regulate redox status in the symbiotic fungus Epichloe festucae (Cartwright and Scott, 2013). We found that the two $R$. irregularis AP1-like transcription factors (RirG251910 and RirG150060) were predominantly expressed in arbuscules (Table S3). Therefore, it is interesting to investigate whether these AP1-like transcription factors control redox-related or ROS detoxification processes in arbuscule cells in a host-dependent manner.

Medicago- and Nicotiana-enriched genes

Among the 172 genes enriched in the two dicot plants (Table S3), we observed an GO-enrichment of genes related to the cytoskeleton (Fig. $5 e$, Table S3). These include for example an alpha tubulin (RirG146020) and a beta tubulin (RirG118260). In addition, many sugar metabolic genes were found to be expressed in a dicot dependent manner (Table S3). These include a glucooligosaccharide oxidase (RirG137100), a glycogen synthase (RirG001390), a glycosyltransferase (RirG192350), an UDP-glucose-6-dehydrogenase (RirG081670) and an alpha-amylase (RirG109680). Moreover, two genes involved in chitin metabolism and biosynthesis, a glucosamine-6-phosphate isomerase 1 (RirG009480) and a chitin synthase export chaperone (RirG071350) are more expressed in the 2 dicots compared to Chives (Table S3), suggesting that AM fungi may undergo specific fungal cell wall remodelling in different hosts. Additionally, we found genes related to iron transport, including the previously mentioned iron transporter RiFTR1 (Tamayo et al., 2018), that are much higher induced in the symbiosis with the two dicot hosts rather than the monocot Chives (Table S3). Interestingly, we found one 


\section{CHAPTER 3}

IucA / IucC gene (RirG008590; RiSID1, Tamayo et al., 2014) which may regulate siderophore biosynthesis that is expressed in a dicot-dependent and ARB-dependent manner (Table S3). It was shown that AM fungi can produce carboxylate siderophores, such as glomuferrin (Winkelmann, 2017). Siderophores are iron-chelating secondary metabolites that are widely used by microbes and plants for iron uptake or maintenance of iron homeostasis. Pathogenic fungi mutated for genes involved in siderophore biosynthetic genes are typically severely impaired in pathogenesis (Verbon et al., 2017; Oide et al., 2006). Moreover, the beneficial effect provided by the mutualistic endophyte Epichloe festucae was impaired after mutation of extracellular siderophore biosynthesis (Johnson et al., 2013). It remains a question whether in $R$. irregularis siderophores are secreted to the apoplast to facilitate ferric acquisition or are used as chelator inside the cell to avoid oxidative stress. In addition, several MFS transporters, including RirG014340, RirG093170, RirG178390 (Table S3) showed higher expression in the symbiosis with Medicago and Nicotiana compared to Chives. All showed enriched expression in ARB and/or IRM compared to ERM or germinating spores. Among all transporter genes, the highest expressed gene is the aquaporin encoding RirG026160, also known as GintAQP1 (Aroca et al., 2009; Table S3). In Rhizophagus clarus an aquaporin, called RCAQP3, was reported to be highly expressed in intraradical mycelium and found to be important for water transport to the host, which also facilitated the long-distance transport of poly-P from the ERM to IRM (Kikuchi et al., 2016). Whether RirG026160 plays a similar role remains to be determined.

\section{Medicago- and Chives-enriched genes}

Among the 116 genes induced by both Medicago and Chives, we observed an enrichment of genes encoding proteins with binding activity (Fig. 5f), notably related to purine nucleotide binding, especially ATP binding. This is likely due to active protein phosphorylation, because the GO-terms kinase activity, phosphorus metabolic process and phosphorylation were enriched (Fig. 5f). 21 out of the 116 enriched genes are annotated as kinases (Table S3).

Nicotiana- and Chives-enriched genes 
Similarly, genes encoding (ATP) binding activity were enriched among the 157 genes with higher expression in both Chives and Nicotiana compared to Medicago (Fig. 5g). Among these we detected 20 kinases (Table S3). Many of them (10 out of 20 ) encode kinases related to the cell cycle, such as cyclin dependent kinases. In addition, we found genes related to glutathione biosynthesis (RirG022970, RirG197620, RirG145750, Table S3) to be enriched in both Chives and Nicotiana. Glutathione is essential to protect cellular components against reactive oxygen species and therefore widely used as antioxidant. Our results suggest that AM fungi may use different ROS detoxifying mechanisms in different hosts. Furthermore, several genes involved in redox balance, particularly several cytochrome P450 genes (RirG088670, RirG072740, RirG026210, RirG087520) were found among the Chives and Nicotiana-enriched genes. Distinct cytochrome P450 genes found in different hosts suggesting that AM fungi adapt to their host environment by rewiring secondary metabolism. In addition, we found several transporters (Table S3), including an amino acid transporter (RirG021210), an arsenite transporter (RirG249510), a nitrate transporter (RirG057900) and a magnesium translocating ATPase (RirG201160) expressed higher in Chives and Nicotiana compared to Medicago, although most of these were weakly expressed.

\section{Stage-dependent transcriptome}

\section{Arbuscules}

Arbuscules are considered to be the main site for nutrient exchange between both partners. In line with this, we observed an enrichment of genes related to the GO-term "intrinsic membrane" (Fig. 6a) and many arbuscule-specific nutrient transporters were revealed (Table S4). This supports the notion that the arbuscule forms a specialized interface to facilitate nutrient exchange. Furthermore, the expression of several genes related to oxidoreductase activity was enriched in arbuscules (Fig. 6a; Table S4). As discussed in the description of the "core symbiotic transcriptome" this may point to specific metabolic processes occurring at this stage of fungal development. Although we tried to isolate mostly arbuscules that filled Medicago cortex cells it is likely that multiple stages of arbuscule development, including the collapse of arbuscule branches, will be represented in the data. Therefore, we cannot distinguish these 
(a) oxidation and reduction
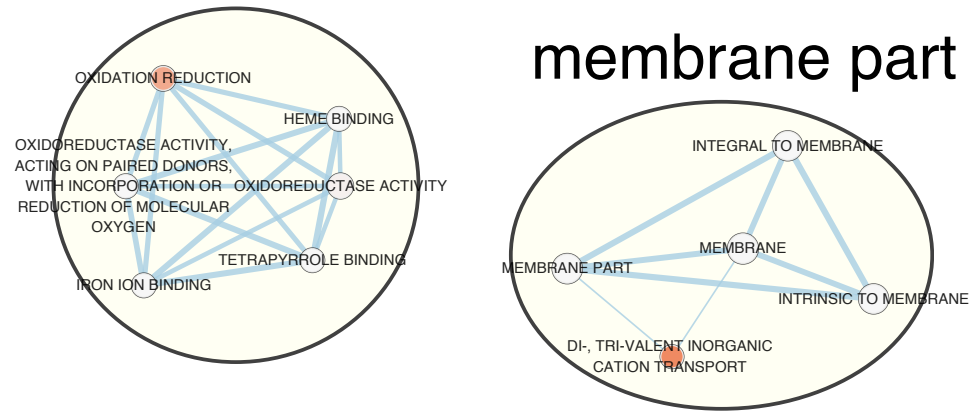

Fig 6. Gene ontology enrichment analysis of Rhizophagus irregularis gene expression in different developmental stages, including arbuscule (a), intraradical mycelium (b), arbuscule and intraradical mycelium (c) and extraradical mycelium (d). Results were generated using Enrichmentmap. Nodes represent gene sets. Node size represents the number of genes in the gene set. Node fill represents enrichment score (q-value). Edge width represents the number of genes that overlap between a pair of gene sets.

continued on next page

different stages of arbuscule development based on our RNA-seq data.

In the top 10 most highly expressed ARB-enriched genes, 9 were predicted to encode for putative effector proteins (Table S4), suggesting a key role for these effectors in arbuscule development and/or function.

\section{Intraradical mycelium}

Among the genes showing enriched expression in IRM, we observed a substantial number of genes involved in cell cycle and pyrimidine metabolic processes (Fig.6b; Table S4). Successful symbiosis requires constitutive nuclear divisions to allow hyphal growth and spore formation both inside and outside of the root. Our results suggest that most nuclear divisions occurred in the IRM, and less in arbuscules. Consistent with this prediction, it was reported that DNA replication mainly occurs in intraradical mycelium and in the thick trunks of arbuscule but not in the arbuscule fine branches (Bianciotto et al., 1995). Nuclei that occupy the arbuscule fine branches were reported to show signs of pyknosis, suggesting that they eventually represent a dead end for the fungus. Surprisingly, we also noticed that GO terms related to cellular response to stress, most notably DNA damage, are also enriched in IRM. Several genes related 
Host- and stage-dependent transcriptome

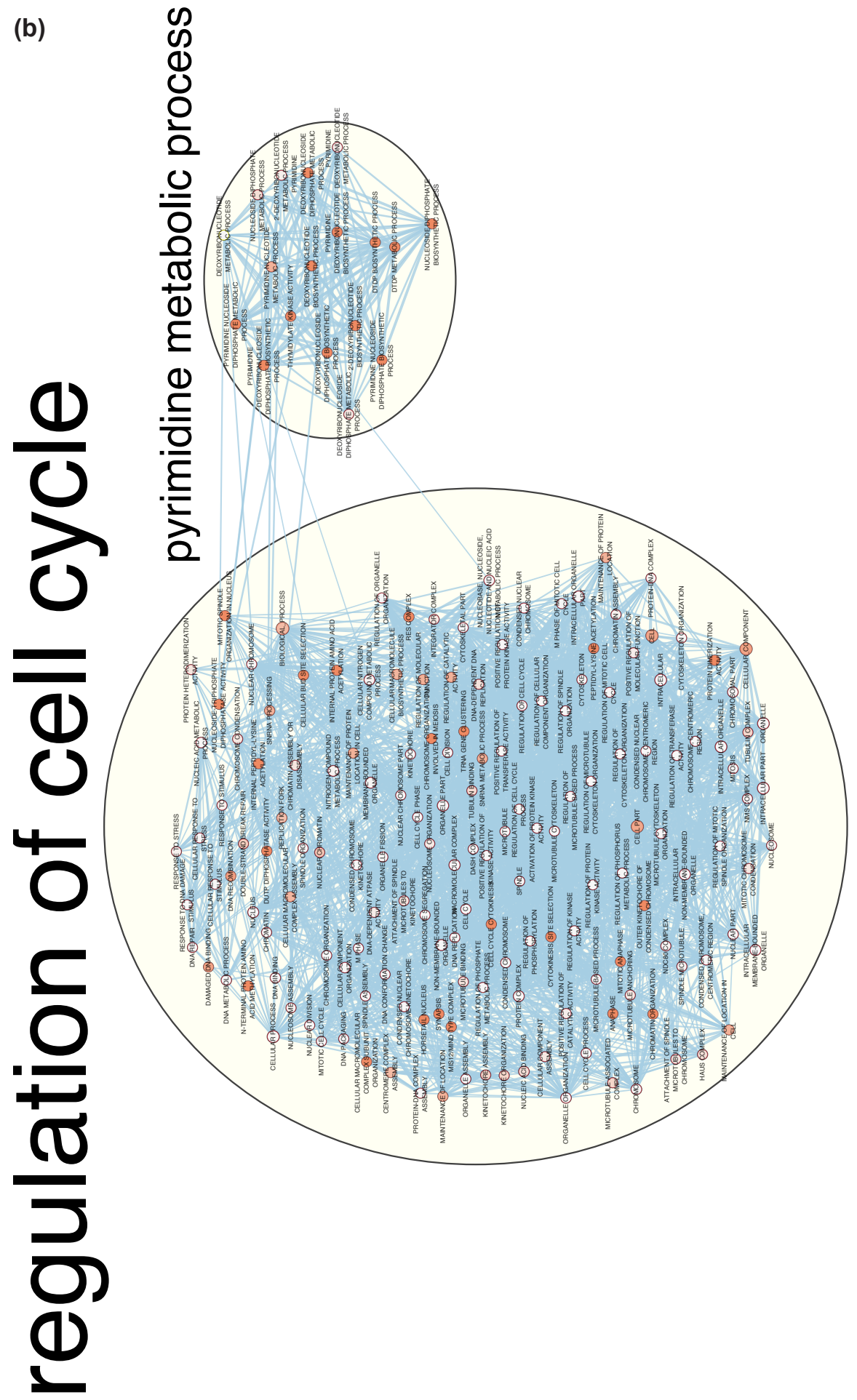


CHAPTER 3

(c)

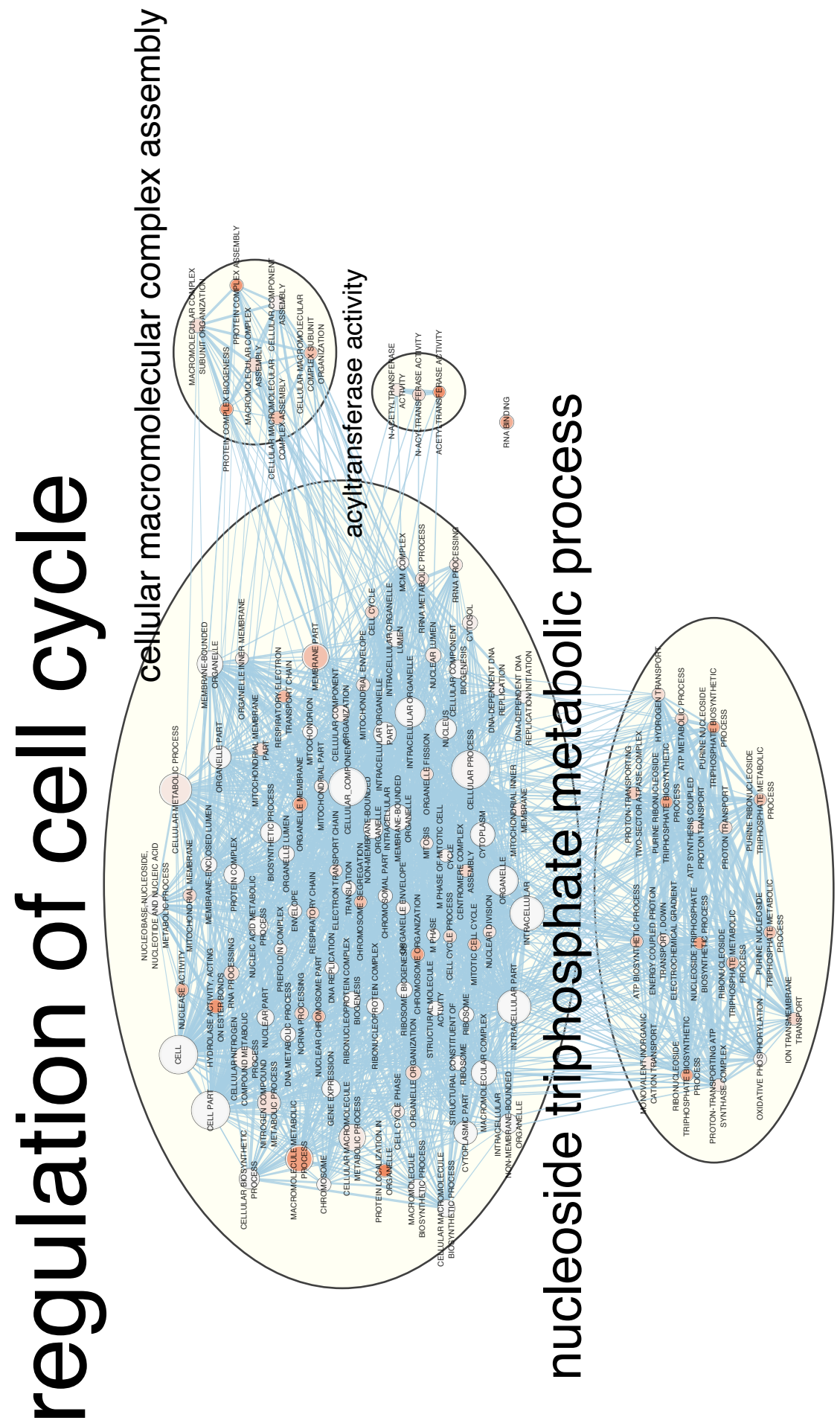




\section{(d) regulation of transcription}

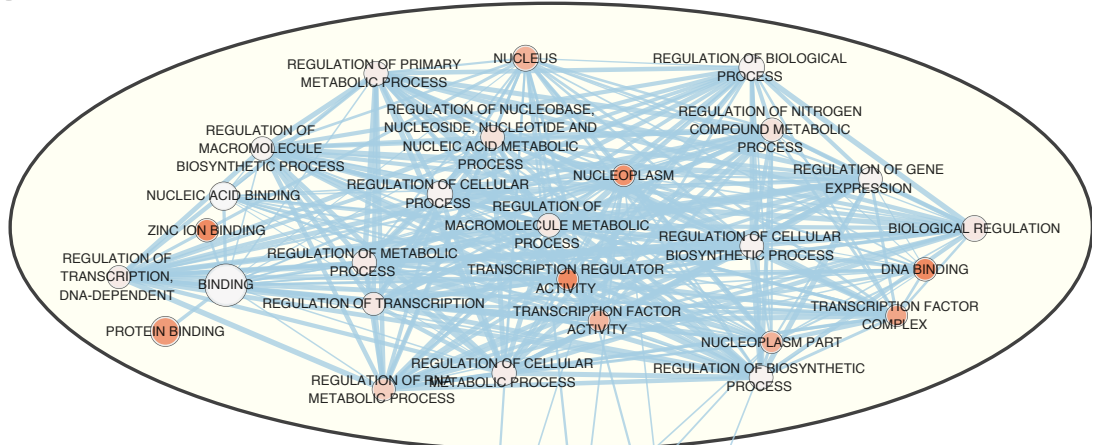

rna polymerase ii dependent transcription

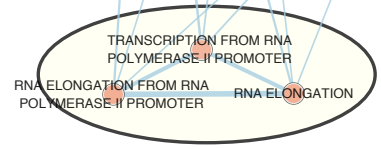

to DNA repair (Fig. 6b) are more prominent in IRM than in the other stages. Whether this is related to a higher nuclear division rate, or active transpon acitivity in IRM or due to a higher exposure to a DNA damaging environment remains to be determined. Interestingly, a recent genome study of Diversispora epigaea (previously Glomus versiforme) revealed that gene families involved in DNA replication and repair are much larger in Glomeromycotina compared to the closely-related Mortierellomycotina and Mucoromycotina as well as several pathogenic fungi (Sun et al., 2018). Therefore, expansion of the number and intensive expression of those genes may be essential for intraradical infection.

In addition to cell cycle related genes, we noticed that genes enriched in both ARB and IRM are often associated with purine biosynthesis activity, possibly related to a higher (ATP) energy demand in those cell types (Fig. 6c). We also observed enriched acetyltransferase activity. 7 out of 10 acetyltransferases are annotated as components the in acyl-carrierprotein (ACP) biosynthetic process, implying an active lipid metabolism in both ARB and IRM. 


\section{CHAPTER 3}

\section{Extraradical mycelium}

Genes with higher expression in ERM are most prominently related to transcriptional regulation (Fig. 6d; Table S4). Many of these genes are associated with molecular functions such as zinc ion binding (Fig.6c). Of the 103 transcriptional regulators enriched in ERM, 26 of them have zinc binding activity (Table S4). This points to an important role for zinc binding transcription factors/regulators to regulate transcription in the ERM.

\section{Concluding remarks}

The transcriptome analyses presented in this chapter provide valuable molecular insight into the host- and stage-dependent regulation of gene expression during the symbiosis. It allowed the identification of numerous transporters expressed at different stages, and identified candidate proteins involved in different (metabolic) process at different stages of the interaction. In the future, these analyses can be extended to include additional stages of AMF development, such as hyphopodia, or various stages of arbuscule development (Kameoka et al., 2018). A very recent study by Kameoka and colleagues performed an RNAseq analysis on different extraradical stages such as branched adsorbing structures and different stages of spore formation (Kameoka, Maeda, et al., 2019). It has become clear that the fungus adjusts its transcriptome depending on the host environment. These adjustments may be related to different immune responses in the various hosts or different metabolic requirements. A challenging next step will be to functionally address the many hypotheses that can be generated based on these data. 


\section{Supplementary data}

A digital version of main figures and supplementary tables listed below can be downloaded from https://1drv.ms/u/s!Ap6ZxoG9Boind8wqDcom WYSHOak?e=pevlep.

Table S1. AM Gene expression data used in this chapter.

Table S2. AM Genes commonly induced in three hosts compared to germinating spores.

Table S3. Host-dependent genes.

Table S4. Stage-dependent genes.

\section{Supplementary Figure}

Upregulated vs GS

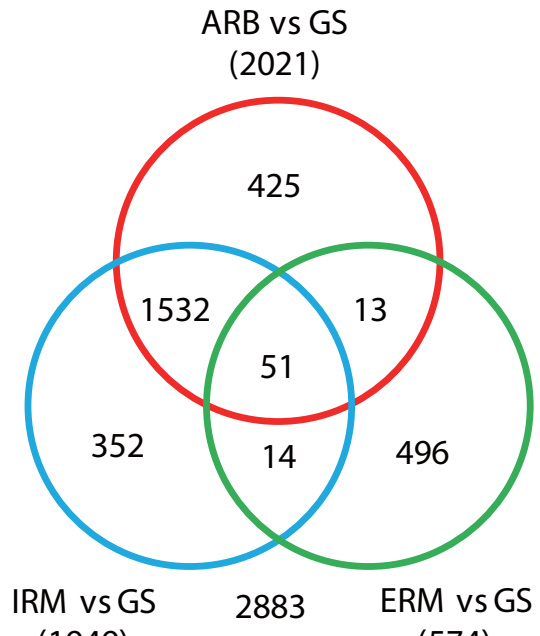

(1949)

(574)

Figure S1. Rhizophagus irregularis genes upregulated in three biotrophic stages arbuscule (ARB), intraradical mycelium (IRM) and extraradical mycelium (ERM) compared to germinating spores (GS). FDR $p<0.05$ and foldchange $>2$. 


\section{CHAPTER 4}

\section{A LysM effector subverts chitin- triggered immunity to facilitate arbuscular mycorrhizal symbiosis}

Tian Zeng ${ }^{1}$, Luis Rodriguez-Moreno ${ }^{2 *}$, Artem Mansurkhodzaev ${ }^{1 * \#}$, Peng Wang ${ }^{1}$, Willy van den Berg ${ }^{3}$, Virginie Gasciolli ${ }^{4}$, Sylvain Cottaz ${ }^{5}$, Sébastien Fort $^{5}$, Bart P.H.J. Thomma ${ }^{2}$, Jean-Jacques Bono ${ }^{4}$, Ton Bisseling ${ }^{1}$, Erik Limpens $^{1}$

1. Laboratory of Molecular Biology, Wageningen University \& Research, 6708 PB Wageningen, the Netherlands

2. Department of Plant Sciences, Laboratory of Phytopathology, Wageningen University \& Research, 6708 PB Wageningen, the Netherlands

3. Laboratory of Biochemistry, Wageningen University \& Research, 6708 WE Wageningen, The Netherlands.

4. LIPM, Université de Toulouse, INRA, CNRS, Castanet-Tolosan, France

5. University Grenoble Alpes, CNRS, CERMAV, UPR 5301, 38041 Grenoble, France

* These authors contributed equally

Corresponding author: erik.limpens@wur.nl

This chapter has been published in New Phytologist.

https://doi.org/10.1111/nph.16245 


\section{CHAPTER 4}

\section{Abstract}

Arbuscular mycorrhizal (AM) fungi greatly improve mineral uptake by host plants in nutrient-depleted soil and can intracellularly colonize root cortex cells in the vast majority of higher plants. However, AM fungi possess common fungal cell wall components such as chitin which can be recognized by plant chitin receptors to trigger immune responses, raising the question how AM fungi effectively evade chitin-triggered immune responses during symbiosis. In this study, we characterize a secreted LysM (Lysin motif) effector identified from the model AM fungal species Rhizophagus irregularis, called RiSLM. RiSLM is one of the highest expressed effector proteins in intraradical mycelium during the symbiosis. In vitro binding assays show that RiSLM binds chitin-oligosaccharides and can protect fungal cell walls from chitinases. Moreover, RiSLM efficiently interferes with chitin-triggered immune responses, such as defence gene induction and reactive oxygen species production in Medicago truncatula. Although RiSLM also binds to symbiotic (lipo)chito-oligosaccharides it does not interfere significantly with symbiotic signalling in Medicago. Host-induced gene silencing of RiSLM greatly reduces fungal colonization levels. Taken together, our results reveal a key role for AM fungal LysM effectors to subvert chitin-triggered immunity in symbiosis, pointing to a common role for LysM effectors in both symbiotic and pathogenic fungi.

\section{Introduction}

Plants interact with microbes that can range from pathogenic to mutualistic. Typically, plants sense the presence of such microbes by the perception of microbe-associated molecular patterns (MAMPs) that are recognized by plant pattern recognition receptors. A major MAMP is chitin, a homopolymer of unbranched $\beta$-1,4-linked $N$-acetylglucosamine which is a key component of fungal cell walls (Valet et al., 2014). Especially its breakdown products chitooligosaccharides (COs) are perceived by plant Lysin motif (LySM) plasma membrane receptors that trigger immune responses such as defence-gene induction, secretion of chitinases and generation of reactive oxygen species (Bozsoki et al., 2017; Miya et al., 2007; Shimizu et al., 2010). Nevertheless biotrophic pathogenic fungi that colonize plants have evolved strategies to evade or down-regulate 
chitin triggered immune responses, especially by secreting specific effectors (Rovenich et al., 2016).

One strategy relies on the secretion of effector proteins that interfere with chitin-triggered immunity (Sánchez-Vallet et al., 2015; Rovenich et al., 2016). These include secreted proteins that consist of 1-3 LysM domains (Kombrink and Thomma, 2013). A key example is the LysM effector Ecp6 from Cladosporium fulvum, which is able to sequester fungal cell wall-derived chitooligosaccharides (COs) (de Jonge et al., 2010). LysM effectors have since been identified in several foliar pathogenic fungi including the wheat pathogen Zymoseptoria tritici (Mycosphaerella graminicola; Mg1LysM and Mg3LysM), the rice blast fungus Magnaporthe oryzae (SIp1), the Brassicaceae anthracnose fungus Colletotrichum higginsianum (ChELP1 and -2) and in the generalist vascular wilt fungal pathogen Verticillium dahliae (Marshall et al., 2011; Mentlak et al., 2012; Lee et al., 2014; Takahara et al., 2016; Kombrink et al., 2017). Such LysM effectors can protect fungal hyphae against the activity of plant chitinases (Mg1LysM and Mg3LysM) or suppresses chitin-triggered defense responses by sequestering chitin fragments (Elp1, Ecp6) or have both activities (Mg3LysM) (Sánchez-Vallet et al., 2015; Rovenich et al., 2016). The occurrence of LysM effectors in such a variety of pathogenic fungi suggests they may be a widespread strategy for fungi to subvert chitin-triggered immunity in a broad range of hosts.

Mutualistic arbuscular mycorrhizal (AM fungi) establish an intimate interaction with the vast majority ( $>80 \%$ ) of all higher plants (Smith and Read, 2008). During this symbiosis, the fungi are intracellularly hosted in the inner root cortical cells forming highly branched hyphal structures, called arbuscules, where nutrients are exchanged in a cooperative manner (Gutjahr and Parniske, 2013). Chitin is also a key component of the cell wall of AM fungi and consistently, it has been reported that plant defence responses are induced in the early stage of AM symbiosis but these are quickly suppressed as the interaction progresses (GianninaziPearson et al., 1996; García-Garrido and Ocampo, 2002; Zamioudis and Pieterse, 2012). How AM fungi suppress chitin induced immune responses is not known. Here we study whether AM fungi use similar effectors as pathogenic fungi to suppress this response.

Genomic analyses have identified a LysM domain containing effector 


\section{CHAPTER 4}

(RiSLM; SECRETED LYSM protein) in the genome of the AM fungus Rhizophagus irregularis DAOM197198 (Tisserant et al., 2013; Sędzielewska Toro and Brachmann, 2016; Zeng et al., 2018; Schmitz et al., 2018). A homolog of this LysM effector, consisting of a single LysM domain, is conserved in a wide-range of $A M$ fungal species and it was recently shown to be able to bind both chitin and chitosan (Schmitz et al., 2019). However, its role in the symbiosis was not investigated. Here, we show that RiSLM one of the most highly expressed effectors in the interaction with an evolutionary diverse range of AM host plants. RiSLM is induced by strigolactones/root exudates and most highly expressed in the intraradical mycelium in Medicago. Our binding studies show that RiSLM can bind chitooligosaccharides in the low micromolar range, as well as lipochitooligosaccharides in the nanomolar range. We show that RiSLM can protect fungal hyphae from plant chitinases, and that it efficiently interferes with chitin-triggered immune responses in the plant. Host-induced gene silencing of RiSLM greatly reduces fungal infection and arbuscule abundance in the host. Taken together, our results indicate that, in analogy to pathogenic fungi, AM fungi use LysM effectors to suppress chitin-triggered immunity to mediate a successful symbiosis.

\section{Materials and methods}

\section{Protein purification}

The coding sequence of RiSLM without signal peptide was amplified using Phusion DNA polymerase (Thermo Fisher Scientific). EcoRI and HindIII restriction sites were added to the primers (Table S1) to facilitate cloning into the $\mathrm{pET}$-SUMO vector (Champion ${ }^{\mathrm{TM}}$ PET SUMO Expression System). The amplified RiSLM CDS was first cloned into the CloneJET vector (Thermo Fisher Scientific) and subsequently cloned EcoRIHindIII into the PET-SUMO vector. All constructs were verified by sanger sequencing. The PET-SUMO vector containing RiSLM was transformed into $E$. coli electrocompetent ORIGAMI cells (genetically modified to allow disulfide bond formation). The bacteria were incubated in LB medium supplemented with $50 \mu \mathrm{g} / \mathrm{mL}$ kanamycin, $10 \mathrm{mM} \mathrm{KH2PO} 4,50 \mathrm{mM} \mathrm{MgSO} 4$ and $1 \%$ glycerol until an OD600 of 0.8 at $28^{\circ} \mathrm{C}$. After that IPTG was added to a concentration of $0.05 \mathrm{mM}$ into the culture and incubated at $28^{\circ} \mathrm{C}$ overnight. Bacterial cultures were then pelleted and stored at $-80^{\circ} \mathrm{C}$. 
For protein purification, frozen bacteria were resuspended in $20 \mathrm{ml}$ lysis buffer (50 mM Tris- $\mathrm{HCl}, 150 \mathrm{mM} \mathrm{NaCl}, 10 \%$ glycerol, $120 \mathrm{mg}$ lysozyme, $40 \mathrm{mg}$ DOC, $1.25 \mathrm{mg}$ DNAse and 1 protease cocktail) per liter culture followed by incubation on ice for 2 hours. The lysate was transferred into $50 \mathrm{~mL}$ tubes and centrifuged for 1 hour at $22000 \mathrm{~g}$ at $4^{\circ} \mathrm{C}$. The supernatant was collected and the SUMO-tagged LysM protein purified by passing through a LP biological system NTA superflow metal affinity chromatography column (Qiagen) according to manufacturer's instructions. Eluted protein was incubated in $200 \mathrm{mM} \mathrm{NaCl}$ in a dialysis membrane with ULP-1 protease to remove the sumo tag overnight at $4^{\circ} \mathrm{C}$. After dialysis the sample was passed again through the NTA column and further purified by size exclusion chromatography using a superdex 75 on a ÄKTA fast-performance liquid chromatographer (GE healthcare, Eindhoven, The Netherlands) using $50 \mathrm{mM}$ Tris- $\mathrm{HCl}, 150 \mathrm{mM} \mathrm{NaCl}(\mathrm{pH}$ 7.4) as elution buffer. Final purified protein was concentrated using an Amicon Ultra-15 centrifugal device (EMD Millipore, Amsterdam, The Netherlands) and the concentration was determined by Qubit (Thermo Fisher Scientific).

\section{Chitin binding affinity precipitation assays}

Purified protein was incubated with $5 \mathrm{mg}$ of insoluble chitin, chitosan, cellulose or xylan (Sigma-Aldrich, Houten, The Netherlands) in $800 \mu \mathrm{l}(1$ $\mu \mathrm{M}$ final concentration of RiSLM) of chitin binding buffer $(50 \mathrm{mM}$ Tris- $\mathrm{HCl}$ $\mathrm{pH} 8.0,150 \mathrm{mM} \mathrm{NaCl}$ ) at room temperature for 3 hours. The tubes were then spun down for $5 \mathrm{~min}$ at $1800 \mathrm{~g}$ and the supernatant was removed. The resulting pellet was washed 3 times with $500 \mu \mathrm{l}$ of chitin binding buffer and resuspended in $200 \mu$ l of SDS loading buffer. The supernatants were concentrated to $250 \mu \mathrm{l}$. $18 \mu \mathrm{l}$ of supernatant was mixed with $6 \mu \mathrm{l}$ of 4 XSDS sample buffer. The pellets and supernatant in SDS were boiled at $95^{\circ} \mathrm{C}$ for $5 \mathrm{~min}$ before loading in a polyacrylamide gelelectrophoresis. Proteins gels were stained by standard Coomassie Brilliant Blue R-250 staining.

\section{Microscale thermophoresis}

Binding experiments were performed by microscale thermophoresis with the Monolith NT.115 (Nano Temper Technologies, Munich, Germany). RiSLM was labelled with the Monolith $\mathrm{NT}^{\mathrm{TM}}$ Protein Labeling Kit RED 


\section{CHAPTER 4}

according to the instructions provided by the manufacturer, using a 1:3 protein:dye molar ratio. For chitooligosaccharide (CO) binding experiments, the labeled protein $(20 \mathrm{nM})$ was incubated with a range of titrant concentrations made by serial dilutions (1:1), in $50 \mathrm{mM}$ Tris buffer $\mathrm{pH} 7.4,10 \mathrm{mM} \mathrm{MgCl} 2,150 \mathrm{mM} \mathrm{NaCl}, 0.05 \%$ Tween 20, in PCR tubes, at room temperature for $10 \mathrm{~min}$. Standard treated capillaries (NanoTemper Technologies, Germany) were loaded and the measurements were performed at $22^{\circ} \mathrm{C}, 20 \%$ LED and $40 \%$ MST power, 20 s laser-on time, $1 \mathrm{~s}$ laser-off time. Due to the physico-chemical properties of the lipochitooligosaccharide (LCO), the above procedure was modified to avoid sticking problems and insertion into micelles. Therefore, a range of LCOs concentrations was prepared by a $1: 1$ serial dilution in $50 \%$ ethanol. The incubation with the labeled protein ( $20 \mathrm{nM})$ was performed in 25 $\mathrm{mM} \mathrm{Na-Cacodylate} \mathrm{buffer} \mathrm{pH} 6.0,1 \mathrm{mM} \mathrm{MgCl} 2,1 \mathrm{mM} \mathrm{CaCl} 2,250 \mathrm{mM}$ sucrose, $0,175 \%$ Fos-Choline-10, $0.5 \%$ ethanol (final concentration), in low binding microtiter plate, at room temperature for $10 \mathrm{~min}$. Premium treated capillaries (NanoTemper Technologies, Munich, Germany) were loaded and the measurements were performed at $22^{\circ} \mathrm{C}$, with the settings used for COs excepted a $40 \%$ LED power. All the experiments were repeated at least twice with two independent protein preparations obtained from two bacterial cultures. Binding data were analyzed using the MO. Affinity Analysis Software (Nano Temper Technologies, Munich, Germany).

\section{Chitinase protection assay}

Either Fusarium oxysporum f.sp lycopersici or Trichoderma viride were used in this assay. In detail, fungal spores were recovered from PDA plates using $2 \mathrm{ml}$ of sterile distilled water. Spore suspension was adjusted to $10^{5}$ spores per $\mathrm{ml}$ and volumes of $40 \mu \mathrm{l}$ were used to fill in a 96 well plate, which was incubated overnight at room temperature. After $\mathrm{o} / \mathrm{n}$ incubation, spore germination was checked by microscopy and treated with $10 \mu \mathrm{L}$ water, SEC buffer, $50 \mu \mathrm{M}$ AVR4, $50 \mu \mathrm{M}$ RiSLM or $100 \mu \mathrm{M}$ RiSLM and incubated at room temperature for 2 hours. After incubation, $10 \mu \mathrm{L}$ of tomato apoplastic fluid preparation were applied into each well and incubated for another 4 hours. Integrity of fungal hyphae were observed using a Leica microscope. Two replicates were used for each treatment.

\section{RNA isolation and qPCR}


For all experiments total RNAs were isolated using a Qiagen plant RNA mini kit or Omega E.Z.N.A Plant RNA kit following the manufacturer's instructions, including on column DNAse treatment. Either $1 \mu \mathrm{g}$ or 500 ng total RNA were used for reverse transcription using iScript cDNA synthesis kit (Bio-Rad). qPCR was done using iQ SYBR Green Supermix (Bio-Rad) on a Bio-Rad CFX realtime platform. All plant and fungal genes were normalized using Medicago truncatula or Rhizophagus irregularis elongation factor 1 a. MQ water was used as negative control in all qPCR analyses. Primers used are shown in Suppl. Table S1.

\section{Defence and symbiotic gene expression analyses}

Medicago truncatula genotype R108 was used. Seeds were surface sterilized and vernalized at $4^{\circ} \mathrm{C}$ for 2 days. 1 -day-germinated seedlings were grown in liquid M medium as described by Nars et al. (2013) for 5 days. M medium was replaced by $M Q$ water before all treatments. In order to study whether LysM effector interferes with CO signalling, 100nM Chitotetraose (CO4, Megazyme), or Chitooctaose (CO8, IsoSep) in combination with either $100 \mathrm{nM}$ or $500 \mathrm{nM}$ LysM effector were used. In order to study whether LysM effector interferes with lipo-chitooligosaccharide (LCO) signalling, 10nM sulfated LCOs (sLCO) or non-sulfated LCOs (nsLCO), in both cases $\mathrm{C} 16: \mathrm{C} 18=1: 1$, in combination with either $10 \mathrm{nM}$ or 50nM RiSLM were used. All samples were harvested at 1 hour post treatment. Three defence marker genes, MtEPI (NAD-dependent epimerase/dehydratase), MtPAL (phenylalanine ammonia lyase) and MtTHA (thaumatin) and three symbiotic marker genes MtPUB1, MTTUBB1 and MtVAPYRIN were used to determine defence or symbiotic responses respectively. For all qPCRs iQ SYBR Green Supermix (Bio-Rad) and the Bio-Rad CFX Real-Time System were used.

\section{Reactive oxygen species (ROS) assay}

Medicago truncatula genotype $\mathrm{A} 17$ was used. Sterilized seeds were grown on Faharaeus medium for 5 days. Roots were cut into $\sim 0.5 \mathrm{~mm}$ pieces and incubated overnight in $150 \mu \mathrm{L} \mathrm{MQ}$ water in a black 96-well polystyrene plate before treatment. Five roots pieces were used in each biological replicate. To ROS, MQ water was replaced by $100 \mu \mathrm{L} M Q$ water containing $0.5 \mathrm{mM} \mathrm{L}-012$ and $10 \mu \mathrm{g} / \mathrm{mL}$ horse radish peroxidase with $1 \mu \mathrm{M}$ CO8. To study the role of RiSLM on ROS generation, equal molar $(1 \mu \mathrm{M})$ 


\section{CHAPTER 4}

RiSLM protein was used. Chemiluminescence was measured using a micro-plate luminometer (CLARIOSTAR, USA) for half an hour. At least 6 biological replicates were used each time and this experiment was repeated independently for 3 times with similar results. Similarly, $100 \mathrm{nM}$ flg22 (Genscript) was applied with or without 400 nM RiSLM.

\section{Host-induced gene silencing}

To generate the silencing construct, $187 \mathrm{bp}$ mRNA sequence of RiSLM was amplified and cloned into the pDONR221 entry vector. Because the CaMV 355 promoter was previously reported to be less active in arbuscule cells, we first replaced the 35 S promoter from the pK7GWIWG2 (II) RR vector (Limpens et al., 2004) by the AtEF1a promoter (Auriac and Timmers, 2007). Next, the silencing fragment of RiSLM was cloned into the modified pK7GWIWG2 (II)-AtEF1a RR vector using LR clonase II (Invitrogen) to get the final silencing construct. Medicago A17 was used for hairy root transformation and the empty vector was used as control. Hairy root transformations were done according to (Limpens et al., 2004). After transformation, composite plants were individually transplanted into SC10 RayLeach cone-tainers (Stuewe and Sons, Canada) with premixed sand:clay (1:2 V/V) mixture. 400 spores of Rhizophagus irregularis DAOM197198 (Agronutrion, France) were used as inoculum for each cone and placed directly below the seedlings upon planting. Plants were watered using half Hoagland medium with full nitrogen as used for our previous research, and grown at in a plant growth chamber at $21{ }^{\circ} \mathrm{C}(16 \mathrm{~h}$ light, 8h dark) (Zeng et al., 2018). After 4 weeks, transgenic roots were harvested based on the DsRed marker expression. Transgenic roots were chopped in to $\sim 2 \mathrm{~cm}$ fragments, mixed thoroughly and separated into 2 parts which were respectively used for microscopy and RNA isolation.

\section{RiSLM overexpression}

For the overexpression of RiSLM, a construct was made using Golden Gate cloning (Engler et al., 2014). Lotus ubiquitin 1 promotor, MtBCP1 signal peptide, RiSLM CDS without SP, and 35S terminator level 0 modules were combined into the level1 position $\mathrm{F} 1$ acceptor vector $\mathrm{pICH} 47732$. A position two AtUB10::DsRed gene was then assembled with the above LjUB1::BCPsp::RiSLM gene into the level2 backbone pAGM4723. The level2 construct was introduced into $A$. rhizogenes MSU440 and then 
transformed into Medicargo plants by hairy root transformation. A Dummy level1 vector (pICH54011) combined with AtUB10::DsRed into pAGM4723 was used as empty vector (EV) control. Plants were harvested after 3 weeks inoculation with $400 R$. irregularis spores as described above.

\section{WGA staining and microscopy}

Mycorrhized roots were first cleared in $10 \%$ potassium hydroxide at $90^{\circ} \mathrm{C}$ for 20 minutes. Then roots were washed twice with PBS buffer (150 $\mathrm{mM} \mathrm{NaCl}, 10 \mathrm{mM} \mathrm{Na} \mathrm{HPO}_{4}$ and $1.8 \mathrm{mM} \mathrm{KH}_{2} \mathrm{PO}_{4}, \mathrm{pH}$ 7.4) followed by incubation in $0.2 \mu \mathrm{g} / \mathrm{mL}$ WGA-Alexafluor 488 in PBS at $4^{\circ} \mathrm{C}$ for overnight. Microscopy was done using a Leica AU5500B microscope. Mycorrhization was quantified according to previously described (Trouvelot et al., 1986).

\section{Phylogeny}

For phylogenetic analyses, amino acid sequences of LysM effectors or LysM domains were aligned using MAFFT plugin in Geneious 8.1.9 (https://www.geneious.com). Phylogenetic tree was generated using tree builder in Geneious 8.1.9 (https://www.geneious.com). For all analyses neighbour-joining tree was used.

\section{Yeast signal sequence trap}

Full length RiSLM was cloned EcoRI-NotI into the pYST-02 vector (Kloppholz et al., 2011). S. cerevisiae strain Y02321 (Mat a; his $3 \Delta 1$; leu2 $\Delta 0$; met15 $\Delta 0$; ura3 $\Delta 0$; YIL162w::kanMX4) (Euroscarf, Germany) was transformed using the standard lithium acetate/single-stranded carrier DNA/PEG method and selected on SD/-Leu plates. Subsequently, transformants were plated on sucrose selection medium $(6.7 \mathrm{~g} / \mathrm{L}$ yeast nitrogen base without amino acids, $1.85 \mathrm{~g} / \mathrm{L}$ Drop-out mix minus leucine, $2 \%$ sucrose, $0.025 \%$ glucose, $2 \%$ Agar) and incubated at $30^{\circ} \mathrm{C}$ for 3 days.

\section{RNAseq analyses}

RNAseq data was collected from the following databases: NCBI gene expression omnibus GSE99655 for data from Medicago, Chives and Nicotiana benthamiana as well as laser microdissected arbuscules (ARB) and IRM; ERM and germinating spores from DDBJ database DRA002591; 


\section{CHAPTER 4}

Lotus japonicus and tomato from DDBJ database DRA005187. RNAseq data was analysed as described by Zeng et al., 2018. Quantification was done using default setting in CLC genomics workbench 10.0.1.

\section{Results}

\section{A Rhizophagus irregularis LysM effector highly expressed in intraradical mycelium}

Fungal pathogens use LysM effectors to counteract chitin-based immune responses to colonize plants (Sánchez-Vallet et al., 2015). By analysing the predicted secretome of the model AM fungus $R$. irregularis DAOM197198 a small secreted effector, consisting only of a single LysM domain, was identified and named RiSLM (Schmitz et al., 2019; Tisserant et al., 2013; Sędzielewska Toro and Brachmann, 2016; Zeng et al., 2018). Our transcriptome analysis showed that this effector is one of the most highly expressed fungal genes in the symbiosis with at least three evolutionarily distantly related plant species, Medicago, Nicotiana benthamiana and Allium schoenoprasum (Zeng et al., 2018). We additionally mined publically available transcriptome data of $R$. irregularis and found that RISLM is also highly expressed in the symbiosis with tomato and Lotus japonicus (Sugimura and Saito, 2017) (Fig. 1a). The high expression level of RiSLM in such a wide range of host species suggests that RiSLM plays a fundamental role during the symbiosis. A time course experiment in Medicago revealed that RiSLM expression correlates with fungal colonization and abundance, as reflected by the expression of $R$. irregularis Elongation Factor $1 a(R i E F)$ and the arbuscule-specific phosphate transporter (MtPT4, Javot et al., 2007) (Fig. 1b). Stagespecific transcriptome analyses in Medicago further indicated that RiSLM is the highest expressed effector gene in intraradical mycelium (Fig. 1c). Expression of RISLM was also induced in germinating spores upon treatment with the strigolactone analog GR24 and rice root exudates (Tsuzuki et al., 2016; Nadal et al., 2017), suggesting that RiSLM may play a role already upon fungal contact at the epidermis. To verify that RiSLM can be secreted by the fungus, we used a yeast signal sequence trap system, in analogy to Kloppholz et al. (2011). Expressing the full length RiSLM protein including its endogenous signal peptide sequence fused to an invertase allowed the mutant yeast strain Y02321, which cannot secrete invertase by itself, to efficiently grow on sucrose containing 


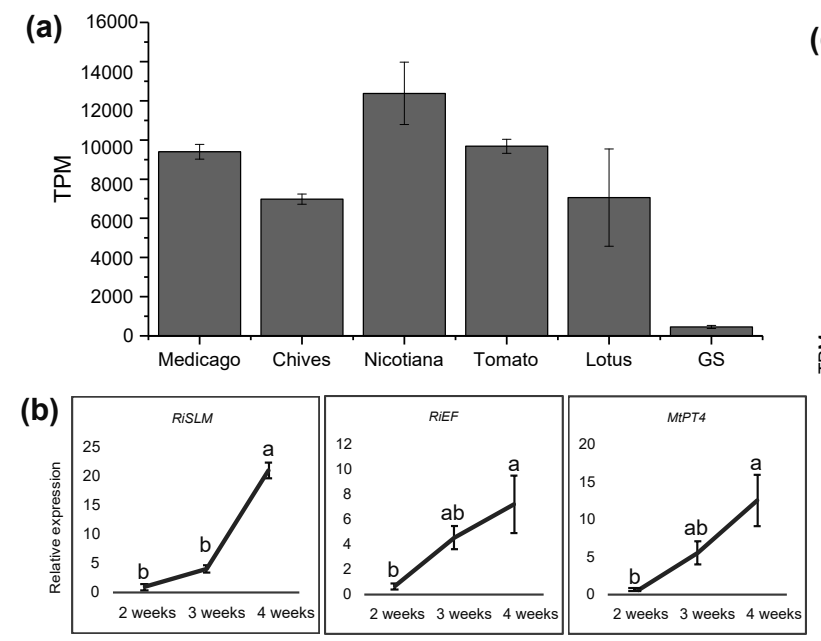

(c)

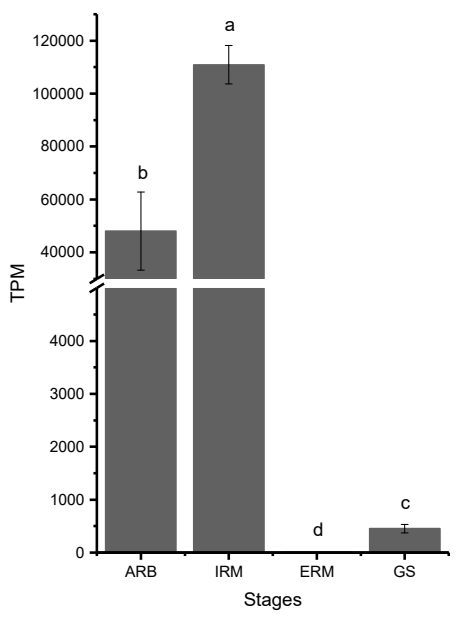

Figure 1. RiSLM is highly induced in intraradical mycelium in a wide range of host plants. (a) RiSLM is highly induced in five hosts Medicago truncatula (Medicago), Allium schoenoprasum (Chives), Nicotiana benthamiana (Nicotiana), Solanum lycopersicum (Tomato) and Lotus japonicus (Lotus) compared to germinating spores (GS). TPM (transcripts per million) was used to represent expression levels. Error bars represent standard error from either 3 replicates (for Medicago, Chives and Nicotiana) or 2 replicates (for Lotus and Tomato), collected from independent RNAseq experiments. (b) qPCR analysis, showing that RiSLM (relative to RiEF) induced during AM colonization and arbuscule development as represented by relative expression of $R$. irregularis Elongation factor (RiEF) and the arbuscule-specific phosphate transporter (MtPT4) normalized by Medicago Elongation factor (MtEF). Error bar represents standard error from three biological replicates. (c) RiSLM expression based on RNAseq analyses of laser microdissected arbuscules (ARB) and intraradical mycelium (IRM) compared to extraradical mycelium (ERM) and germinating spores (GS). Error bars represents standard errors from three biological replicates. For all figures, different characters indicate significant differences (LSD $p<0.05)$

medium (Fig. S1). This indicates that RiSLM is indeed a secreted protein.

\section{LysM effector genes are a conserved feature in AM fungi}

To determine whether the presence of a LysM effector gene is a conserved feature of AM fungi, we searched for RiSLM homologs in the publically available genome or transcriptome sequences of 5 additional $R$. irregularis isolates (Ropars et al., 2016; Chen et al., 2018) and of the different AM species Rhizophagus clarus (Sędzielewska Toro and Brachmann, 2016), Gigaspora margarita and Gigaspora rosea (Salvioli et al., 2016; Tang et al., 2016). In all AM fungal genomes/transcriptomes we could detect 


\section{CHAPTER 4}

the presence of LysM effectors, containing a signal peptide followed by a single LysM domain (Table S2). Most AM fungal species/isolates harbour only one LysM effector. However, we detected four LysM effector-coding genes in the $R$. irregularis A4 genome (Fig. 2). We noticed that there is significant variation at the RiSLM amino-acid level in the different $R$. irregularis isolates, in agreement with the analyses by Schmitz et al. (2018). Strikingly, even higher divergence is observed among RiSLM protein sequences of different $R$. irregularis isolates than between the LysM effector from $R$. irregularis and G. rosea (Fig. 2), which leads us to speculate that there may be significant levels of selection or adaptation to different plant hosts, for example to avoid subsequent effectortriggered immune responses. Nevertheless, these analyses indicate that the presence of a LysM effector gene is a conserved feature in AM fungi.

Comparison of AM fungal LysM domains with pathogenic fungal LysM domains showed that most AM LysM effectors contain two conserved cysteine residues in the LysM domain (Fig. S2), in contrast to only one or no cysteines in the LysM domains of pathogenic LysM effectors. These cysteine residues may be crucial to form disulfide bridges to stabilize the protein or to form protein complexes. In addition, significant enrichment of positively charged lysine and arginine residues was observed in most AM LysM domains (Fig. S2), suggesting that ionic interactions may be important for proper function of AM LysM effectors. Alternatively, positive charged residues in the LysM domain may be required to recruit the effectors to the negatively charged host membrane to interact with potential host receptors.
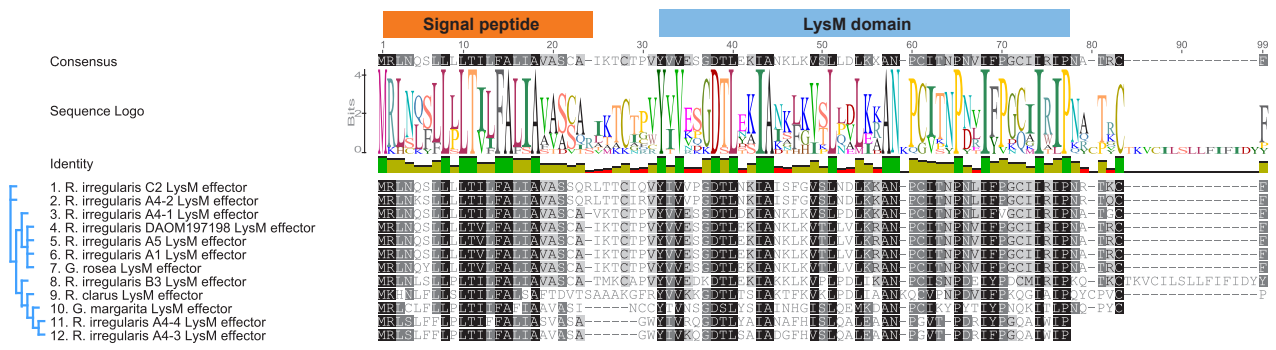

Figure 2. LysM effectors are a conserved feature of AM fungi. LysM effectors from different AM fungal species/isolates were aligned using MAFFT and grouped using Geneious tree builder (https://www.geneious.com). Signal peptide and LysM domain are marked. 


\section{RiSLM binds chitin and chitooligosaccharides}

To investigate the function of RiSLM we produced and purified the RiSLM protein, using the Champion pET-SUMO expression system, from Escherichia coli ORIGAMI cells. After affinity purification and size-exclusion chromatography we noticed two faint additional bands (of $\sim 14 \mathrm{kDa}$ and $\sim 21 \mathrm{kDa}$ ) next to the expected $\sim 7 \mathrm{kDa}$ RiSLM band on a coomassie blue stained protein gel (Fig. S3a). These bands may reflect oligomers of RiSLM, as was previously observed for the SIp1 LysM effector from $M$. oryzae and Vd2LysM from V. dahliae (Mentlak et al., 2012; Kombrink et al., 2017). We first tested the ability of the purified RiSLM protein to bind chitin using affinity precipitation assays with the insoluble carbohydrates chitin, chitosan, xylan and cellulose. This showed that the majority of the RiSLM protein was precipitated with insoluble chitin, but not (or hardly) with cellulose or xylan (Fig. S3b). Incubation with insoluble chitosan (deacetylated chitin) also resulted in precipitation of RiSLM although not as strong as with chitin. This indicates that RiSLM has the highest affinity for acetylated chitin residues. To better study the affinity of RiSLM for chitooligosaccharides (COs) of different lengths we performed microscale thermophoresis experiments. This revealed a binding affinity $\left(K_{d}\right)$ of 8.8 $\pm 1.2 \mu \mathrm{M}$ for $\mathrm{CO} 7$, and slightly lower for CO4 $(37.1 \pm 1.4 \mu \mathrm{M})$ and $\mathrm{CO} 5$ $(59.4 \pm 2.1 \mu \mathrm{M})$ (Fig. 3). No binding was observed for their deacetylated

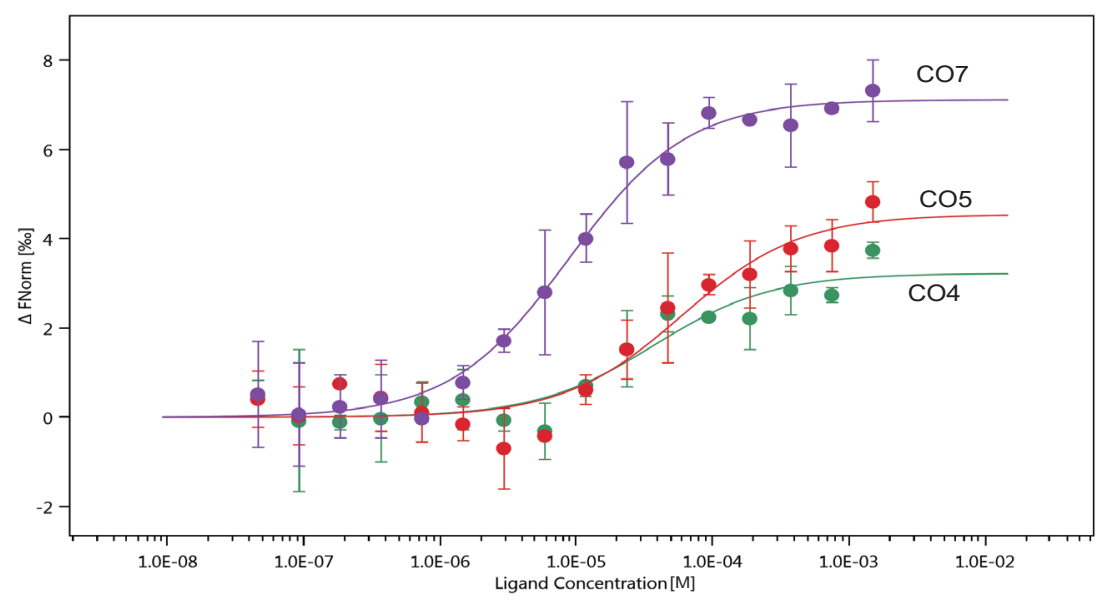

Figure 3. RiSLM binds chitin in the micromolar range. $\mathrm{CO} 4, \mathrm{CO} 5$ and $\mathrm{CO} 7$ were used to test binding affinity between RiSLM and chitin-oligosaccharides using microscale thermophoresis. This revealed affinities of RiSLM for CO4, CO5 and $\mathrm{CO} 7$, respectively. $X$ axis indicates ligand concentration. $Y$ axis indicates the change in normalized fluorescence ( $\Delta$ Fnorm in $[\%]$ ]) after binding with different ligands. 


\section{CHAPTER 4}

counterpart (Fig. S4). These analyses show that RiSLM can bind COs of various lengths, of which especially longer chain $\mathrm{CO}$ such as $\mathrm{CO} 7$ or $\mathrm{CO} 8$ are known to be potent elicitors of chitin triggered immunity in plants.

\section{RiSLM can protect fungal hyphae against degradation by plant chitinases}

Fungal LysM effectors from pathogens have been found to either protect fungal cell walls from apoplastic chitinases or to suppress chitin-triggered immunity, with some LysM effectors possessing both activities to support fungal infection (de Jonge et al., 2010; Marshall et al., 2011; Mentlak et al., 2012; Lee et al., 2014; Kombrink et al., 2017). To test whether RiSLM has the ability to protect against plant chitinases, we tested the effect of RiSLM on hyphal integrity of Trichoderma viride and Fusarium oxysporum f. sp. lycopersici upon treatment with tomato chitinases. Germinated $T$. viride and $F$. oxysporum f. sp. lycopersici spores were incubated for 4 hours with a chitinase extract from tomato leaves in presence or absence of 50 or $100 \mu \mathrm{M}$ RiSLM. As a positive control we used the chitin-binding AVR4 effector from Cladosporium fulvum, which has been shown to protect fungal hyphae in a similar assay (van den Burg et al., 2006). As can be seen from Fig. 4 and Fig. S5, fungal hyphae were still intact after treatment with the chitinase extract in the presence of both CfAVR4 and RiSLM, while control treatments resulted in their degradation, showing that RiSLM can protect fungal hyphae against the hydrolytic activity of plant chitinases.

\section{RiSLM suppresses chitin-triggered immune responses}

Next we tested whether RiSLM can also suppress chitin triggered immune responses in the plant. As a first assay we studied the ability of RiSLM to suppress CO8-induced defence gene induction in Medicago roots. In this assay (Nars et al., 2013), freshly germinated Medicago seedlings were incubated in liquid $M$ medium for 5 days at $21^{\circ} \mathrm{C}$ after which the plants were treated with $100 \mathrm{nM}$ CO8 in the presence or absence of either equimolar ( $100 \mathrm{nM})$ or $5 \times$ excess $(500 \mathrm{nM})$ of RiSLM protein. Roots were harvested 1 hour after treatment and the expression of three defence marker genes, MtEPI (NAD-dependent epimerase/dehydratase), MtPAL (phenylalanine ammonia lyase) and MtTHA (thaumatin), was determined by real-time PCR analysis. Upon 1 hour CO8 treatment all marker genes 


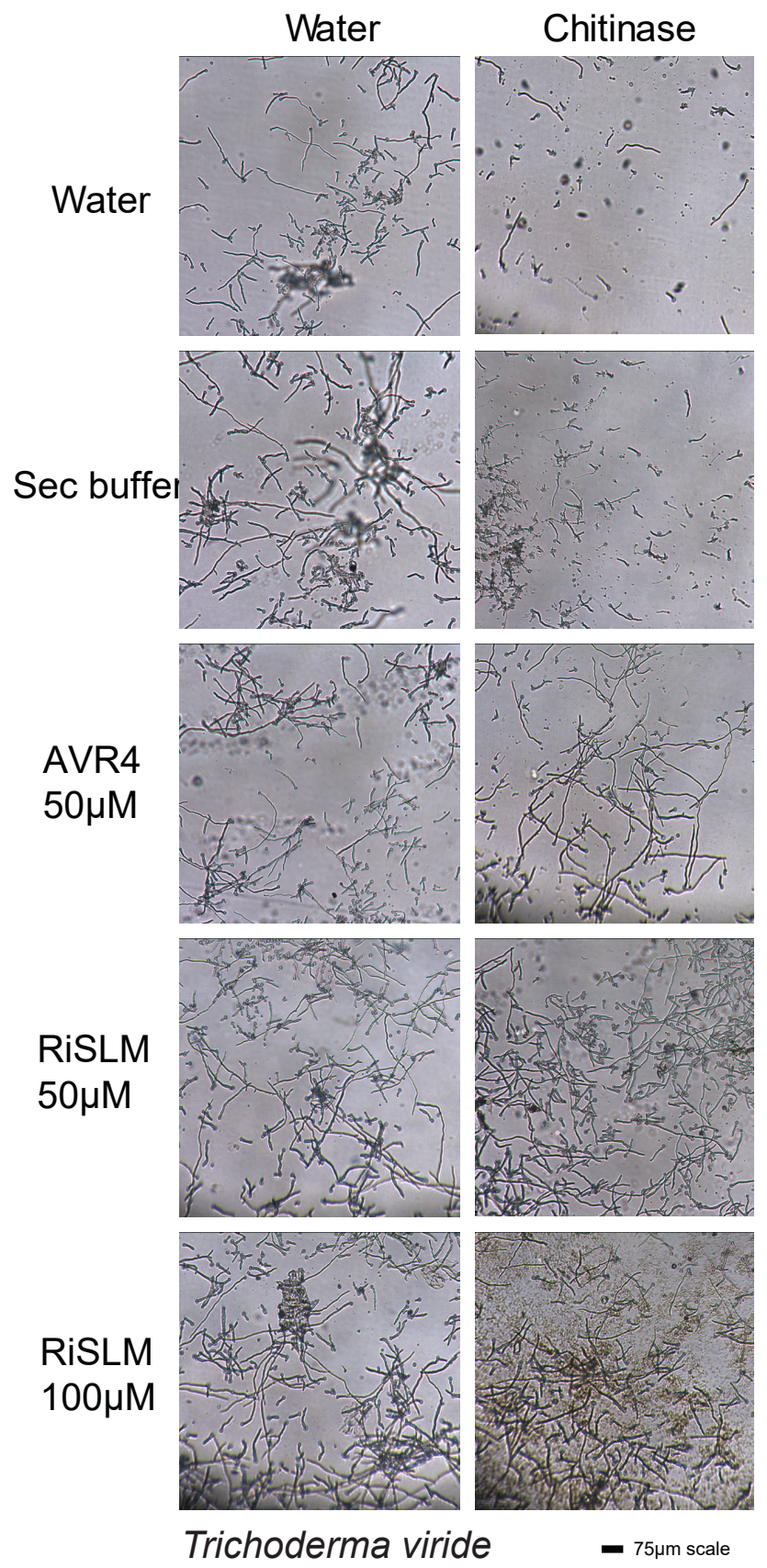

Figure 4. RiSLM protects hyphae from the pathogenic fungus Trichoderma viride against plant chitinases. Germinated hyphae are treated with $50 \mu \mathrm{M}$ AVR4, $50 \mu \mathrm{M}$ RiSLM or $100 \mu \mathrm{M}$ RiSLM and incubated at room temperature for 2 hours, and subsequently treated with a tomato chitinase preparation or water for 4 hours. Water or buffer used for size-exclusion chromatography (SEC) were used as negative controls. The Avr4 effector from Cladosporium fulvum was used as positive control. 


\section{CHAPTER 4}

were markedly induced. This induction was suppressed by $100 \mathrm{nM}$ RiSLM, with $500 \mathrm{nM}$ RiSLM showing an even stronger inhibitory effect, indicating that RiSLM suppresses CO8-induced defence gene expression in a dosedependent manner (Fig. 5a).

As a second assay we measured the ability of RiSLM to inhibit CO8-induced reactive oxygen species (ROS) production using a chemiluminescence assay. In the absence of RiSLM, $1 \mu \mathrm{M}$ CO 8 induced a clear ROS burst in $0.5-1 \mathrm{~cm}$ Medicago root sections (Fig. 5b). However, the ROS burst was greatly reduced upon co-treatment with equimolar $(1 \mu \mathrm{M})$ amounts of RiSLM, showing that RiSLM is able to suppress chitin-triggered ROS production (Fig. $5 \mathrm{~b}$ ). To rule out that RiSLM may have a general inhibitory effect on PAMP-triggered defence responses, we tested whether RiSLM would inhibit flg22-induced ROS production. No inhibition of a ROS burst induced by $100 \mathrm{nM}$ flg 22 was observed in Medicago roots when 4x excess RiSLM was used (Fig. S6). Taken together, these results indicate that RiSLM can suppress chitin-triggered immune responses.

\section{RiSLM does not significantly block symbiotic signalling}

Besides chitin oligomers that trigger immune responses, AM fungi also produce chitin-derived lipo-chitooligosaccharides (Myc-LCOs) and short chain COs (CO4/5), collectively called Myc factors, that activate the common symbiotic signalling pathway to establish the symbiosis (Maillet et al., 2011; Genre et al., 2013). Our study already indicated that RiSLM can bind $\mathrm{CO} 4$ and $\mathrm{CO} 5$ in the micromolar range (Fig. 3), raising the question whether RiSLM might interfere with the signalling role of Myc factors. To investigate this we first additionally tested the affinity of RiSLM for Myc-LCOs. R. irregularis produces both sulphated and nonsulphated tetrameric or pentameric LCOs with variable length/saturation of the fatty acyl chain (Maillet et al., 2011). Microscale thermophoresis analyses of RiSLM with either sulphated or non-sulphated tetrameric (C18:1) LCOs showed that RiSLM binds these Myc-LCOs. LCO-IV(C18:1, S) binding curve analysis revealed two binding events (inset Fig. 6a). The first one occurring at concentrations ranging from 0.1 to $100 \mathrm{nM}$ while the other one was observed at higher LCO concentrations, with a much larger fluorescent signal amplitude but without reaching saturation. LCO-IV(C18:1) binding to RiSLM resulted in a single binding event exhibiting a feature similar to the first event of $\operatorname{LCO}-\operatorname{IV}(C 18: 1, \mathrm{~S})$ binding. 
(a)

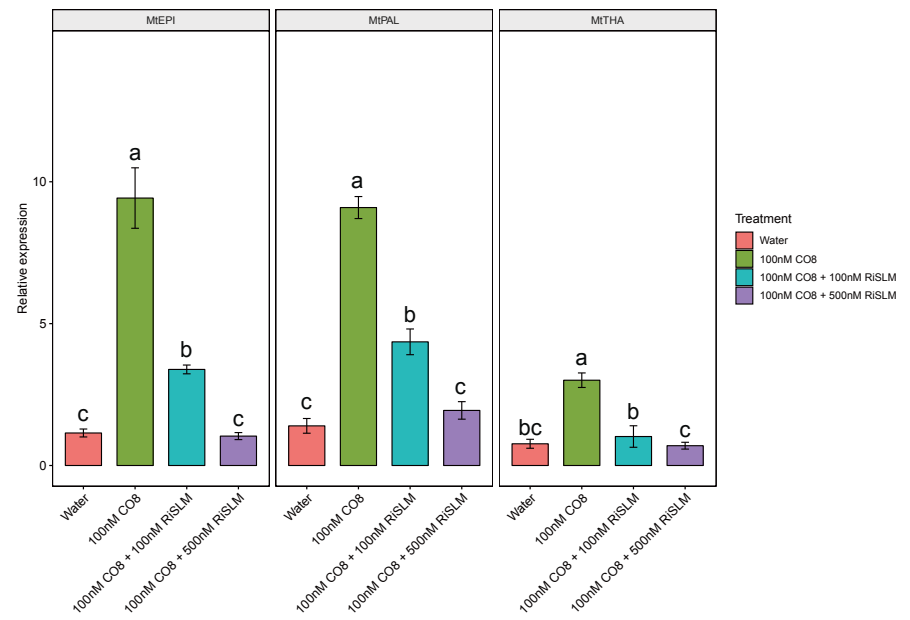

(b) 60000

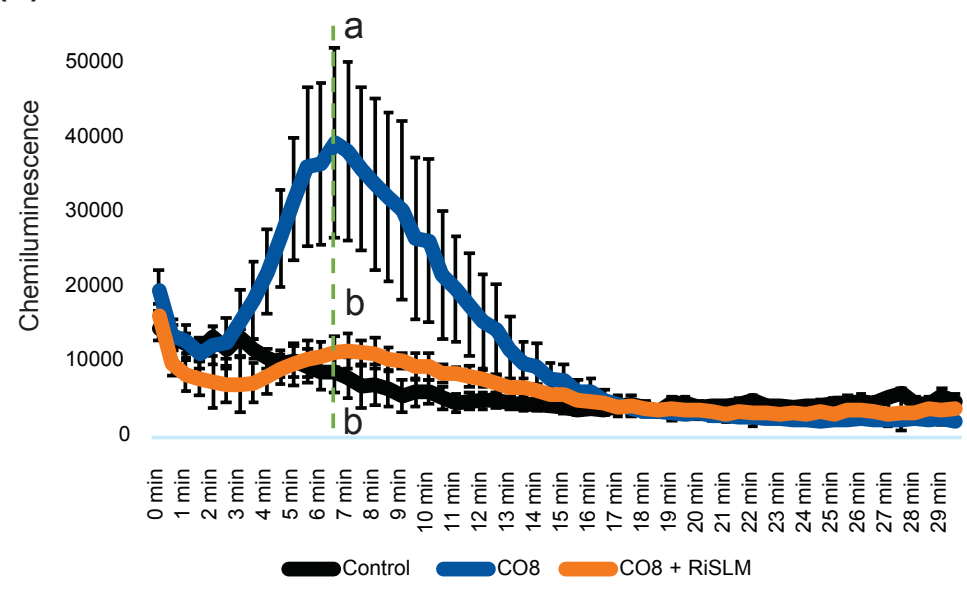

Figure 5. RiSLM suppresses CO8-triggered plant immunity. (a) RiSLM suppresses CO8-triggered defence marker gene induction in Medicago R108 roots treated with $100 \mathrm{nM}$ CO8 in combination with either $100 \mathrm{nM}$ or $500 \mathrm{nM}$ RiSLM for 1 hour. Three defence marker genes EPI, PAL and THA were used to monitor defence responses by qPCR. Error bar represents standard error from three biological replicates. (b) RiSLM suppresses chitin-triggered ROS burst. Medicago truncatula A17 root pieces were treated with $1 \mu \mathrm{M}$ CO8 with or without equal molar amounts of RiSLM . Error bars represent standard error from 6 biological replicates. For both figures, different letters indicate significant difference (LSD $p<0.05)$ between different treatments.

Therefore, by referring to the same binding event, it can be deduced from the plots reported in Fig.6a (main panel) that LCO-IV (C18:1, S) exhibits a higher affinity $\left(K_{\mathrm{d}} \sim 15 \mathrm{nM}\right)$ than LCO-IV(C18:1) $\left(K_{\mathrm{d}} \sim 240 \mathrm{nM}\right)$. The second binding event observed for LCO-IV(C18:1, S) could be due to multimerization, and because it occurs at high concentrations it is 
(a)

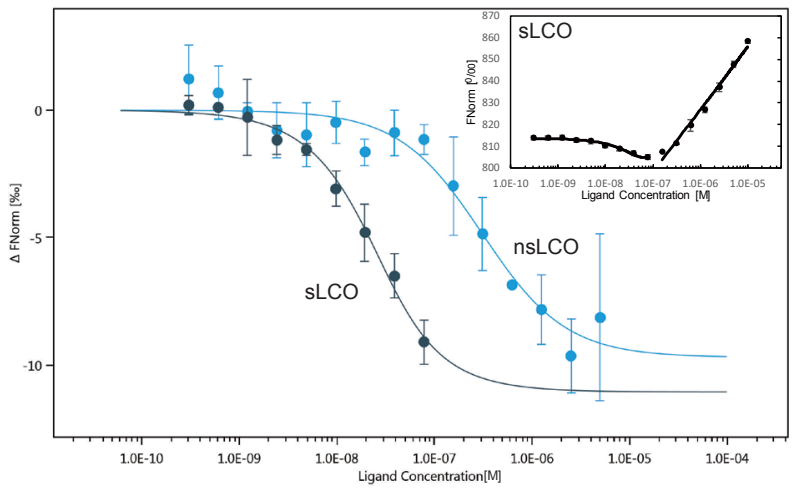

(b)

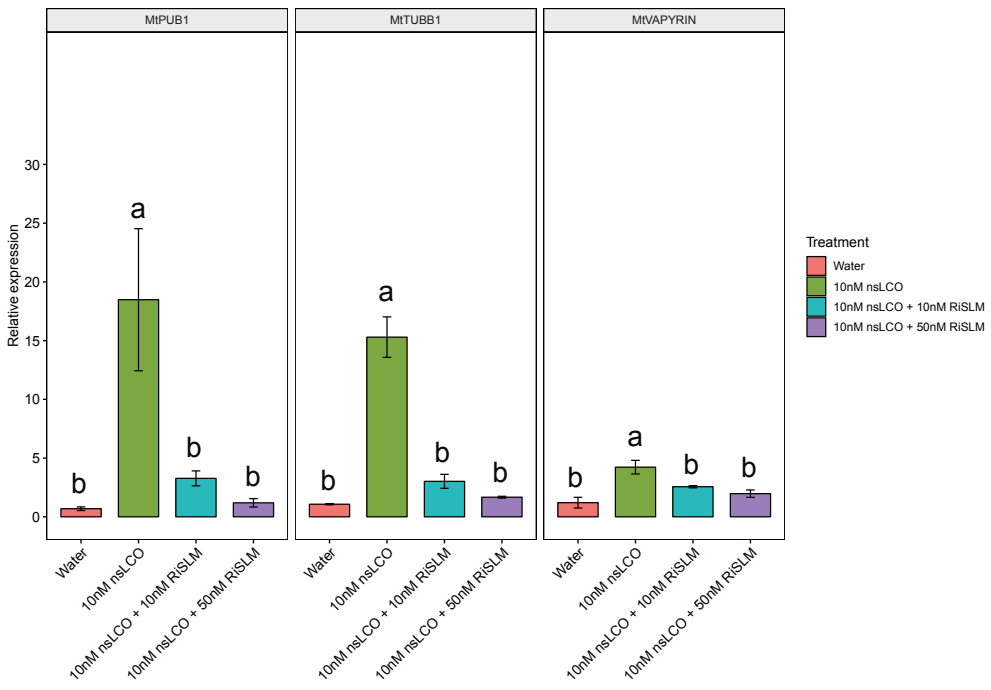

continued on next page

probably not biologically relevant.

Next we tested whether co-application of RiSLM can interfere with symbiotic marker gene induction by the different Myc factors. Therefore, we used the same experimental set-up as before for monitoring the defence gene induction by $\mathrm{CO} 8$ and roots were harvested 1 hour after Myc factor application. For Myc-LCO application, equal molar amounts of C16:0 and C18:1 sulphated LCO were mixed and used as Myc-sLCO (10 nM) preparation and the same was done for $\mathrm{C} 16: 0$ and $\mathrm{C} 18: 1$ nonsulphated LCOs (Myc-nsLCO). For CO4 we used 100 nM, and in all cases RiSLM was applied in equimolar amounts as well as in a $5 \times$ excess. To quantify symbiotic responses, three marker genes (MtPUB1, MtTUBB1 and MtVAPYRIN) were selected that have previously been shown to be 
(c)

(c)

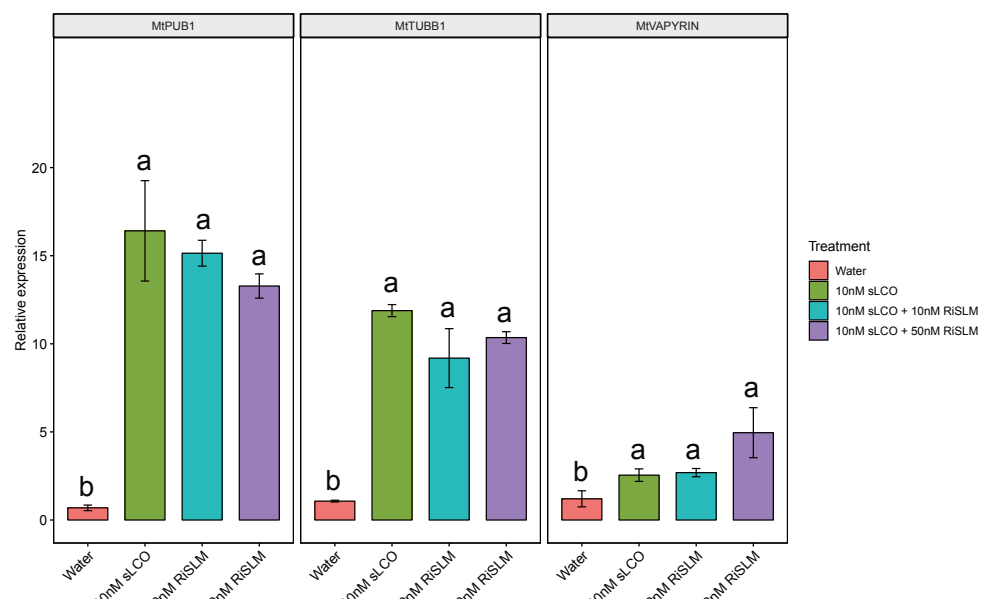

(d)

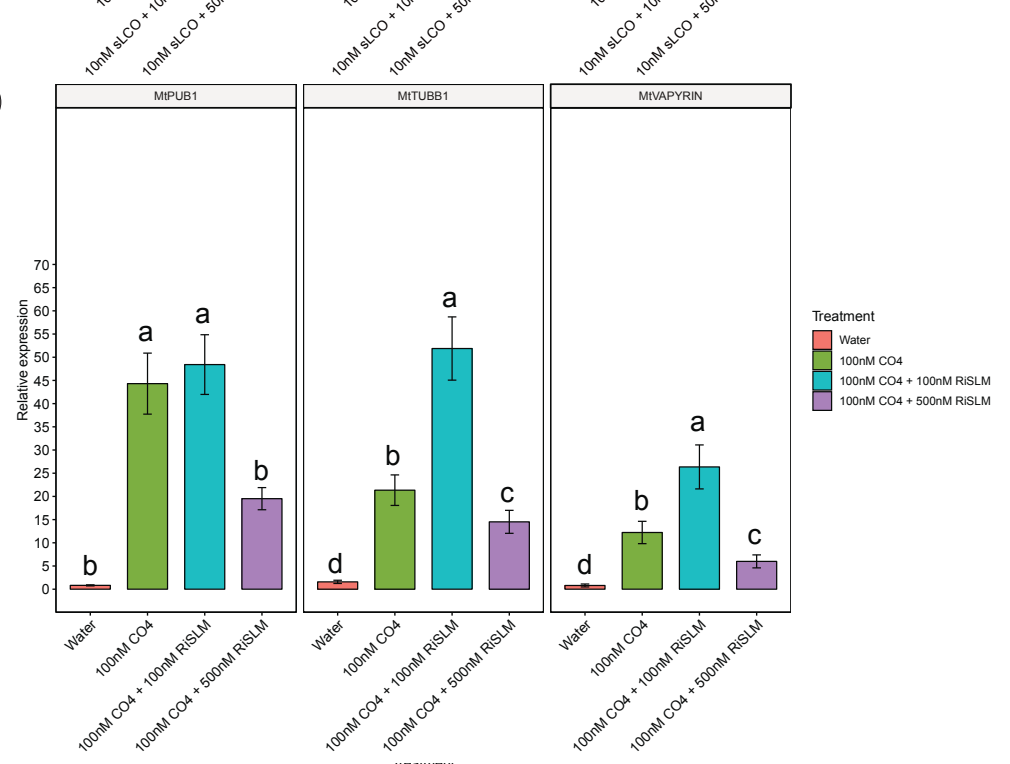

Figure 6. RiSLM does not block symbiotic responses. (a) Binding of RiSLM to LCO-IV(C18:1, S) and LCO-IV(C18:1) (black and blue circles respectively) as determined by microscale thermophoresis and corresponding to the same binding event. Inset shows the two binding events occurring at low $(0.1$ to $100 \mathrm{nM})$ and high concentrations (100 nM to $10 \mu \mathrm{M}$ without reaching saturation) of LCO-IV(C18:1, S) (b) QPCR analysis of Medicago R108 roots treated with $10 \mathrm{nM}$ non-sulphated myc-LCO's (b) or sulphated myc-LCO's (c) in combination with either 10nM or 50nM RiSLM, Three symbiotic marker genes PUB1, TUBB1 or Vapyrin were used to monitor symbiotic responses induced by LCOs, using Medicago EF as reference gene. Error bar represent three biological replicates. (d) qPCR analysis of Medicago R108 roots treated with water $(n=3)$ or $100 \mathrm{nM} \mathrm{CO4}(n=3)$ in combination with either $100 \mathrm{nM}(n=4)$ or $500 \mathrm{nM}$ RiSLM $(n=3)$, showing that RiSLM does not strongly suppress CO4-triggered symbiotic marker gene induction. Different letters indicate significant difference (LSD $p<0.05)$ between different treatments. 


\section{CHAPTER 4}

induced by Myc-LCOs (Camps et al., 2015; Czaja et al., 2012). MtPUB1 encodes a LCO-induced ubiquitin E3 ligase, which interacts with LYK3 and DMI2 receptor kinases to limit rhizobial and AM fungal infection (Vernié et al., 2016). VAPYRIN is thought to be involved in the regulation of exocytosis required for colonization and widely used as a symbiotic marker (Murray et al., 2011; Pumplin et al., 2010). The Myc-LCOs induced MtTUBB1 encodes a putative $\beta$-tubulin (Camps et al., 2015; Czaja et al., 2012). Our results indicated that RiSLM already strongly inhibited nsLCOtriggered symbiotic gene induction when applied at equimolar amounts (Fig. 6b). However, RiSLM did not show any inhibition of sLCO- triggered symbiotic responses, even when applied at 5 times higher levels (Fig. 6c). For $\mathrm{CO} 4$ we observed an $\sim 50 \%$ reduced induction for MtPUB1 and MTVAPYRIN and $\sim 30 \%$ reduced induction for MTTUBB1, when applied in excess (Fig. 6d). However, co-application of RiSLM with equimolar amounts of CO4 strikingly enhanced the expression of MtTUBB1 and MtVAPYRIN by a factor 2.5 .

Taken together, our data indicate that RiSLM can suppress and help evade chitin-triggered immune responses while still allowing sufficient symbiotic signalling to colonize the roots.

\section{RiSLM plays a positive role in root colonization}

To determine whether RiSLM indeed has a positive role during root colonization, we attempted to overexpress RiSLM or to knock-down its expression via host induced gene silencing (HIGS). Although AM fungi currently cannot be stably transformed due to their coenocytic nature, HIGS has previously been successfully used to study the function of several fungal genes (Helber et al., 2011; Kikuchi et al., 2016; Tsuzuki et al., 2016).

To determine whether overexpression of RiSLM could increase colonization, we overexpressed RiSLM in Medicago roots under control of the AtUBIQUITIN3 promoter. We noticed that expression of the full length predicted $R$. irregularis effectors containing their endogenous signal peptides in planta, typically leads to an accumulation in the ER (data not shown). Therefore, to efficiently direct the effector to the apoplast, we fused the mature RiSLM protein to the signal peptide of MtBCP1 (Ivanov and Harrison, 2014). We confirmed the overexpression 
of RiSLM by real-time PCR (Fig. S7b). Three weeks after inoculation no significant differences in either fungal abundance or arbuscule abundance was observed compared to empty vector control roots (Fig. S7a, c). This likely suggests that the endogenous levels of RiSLM expression are sufficient to support successful infection, although we did not verify the overexpression at the protein level.

To trigger HIGS, we introduced a hairpin construct targeting RiSLM driven by the constitutive AtEF1alpha promoter (Auriac and Timmers, 2007) into Medicago roots via Agrobacterium rhizogenes-mediated transformation. An empty vector was used as control. Transgenic roots were selected based on the co-expression of a DSRED1 marker gene. Four weeks after inoculation with $R$. irregularis spores the level of AM colonization was scored (Trouvelot et al., 1986) in transgenic roots stained with WGAAlexa Fluor 488. This showed that roots carrying the silencing construct had significantly lower fungal colonization and arbuscule levels compared with empty vector control roots ( $F i g .7 a, c, d$ ). This correlated with reduced expression of RiEF, a marker for total fungal abundance, and expression of the arbuscule-specific phosphate transporter MtPT4 used as marker for arbuscule abundance (Javot et al., 2007) (Fig. 7b). Consistently, RiSLM expression was also significantly reduced in the HIGS roots compared with empty vector control roots (Fig. 7b). This indicates that RiSLM plays a positive role in AM fungal root colonization.

\section{Discussion}

Here we show that the AM fungus Rhizophagus irregularis secretes the chitin-binding LysM effector RiSLM to facilitate efficient intraradical colonization. A secreted single LysM domain containing effector is present in a wide range of $A M$ species, suggesting that the involvement of a LysM effector is a conserved feature in AM fungi. The variation in protein sequence of these LysM effectors in different isolates, suggests a potential diversifying selection which has also been reported for LysM effectors from pathogenic fungi (Marshall et al., 2011; Schmitz et al., 2019). Purified RiSLM was able to protect fungal hyphae from hydrolysis by plant chitinases and to suppress chitin-triggered immune responses in Medicago roots. A combination of both activities has also been reported for 


\section{CHAPTER 4}

(a)

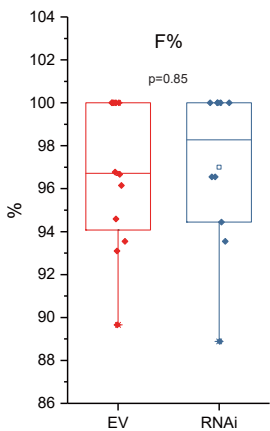

(b)

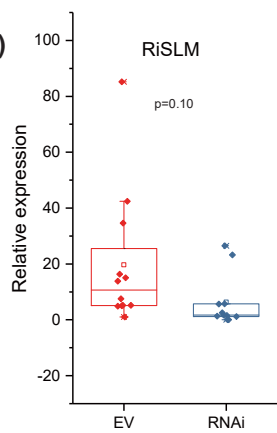

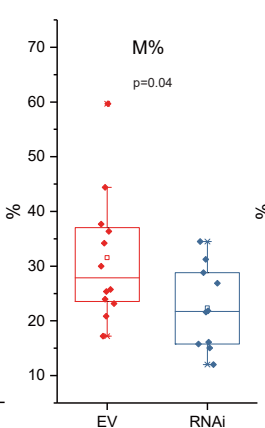
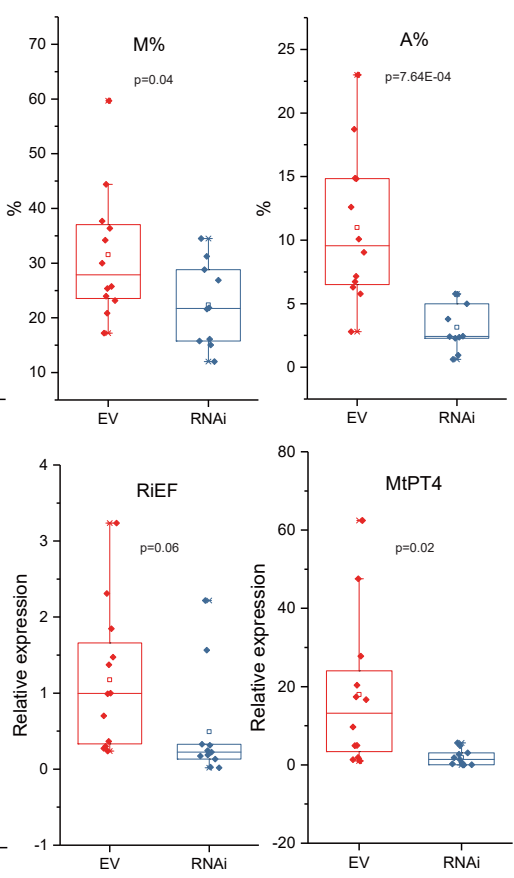

(c)

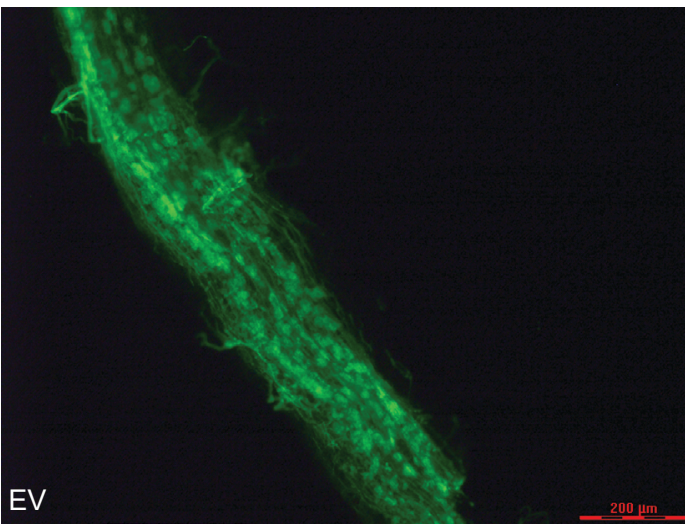

(d)

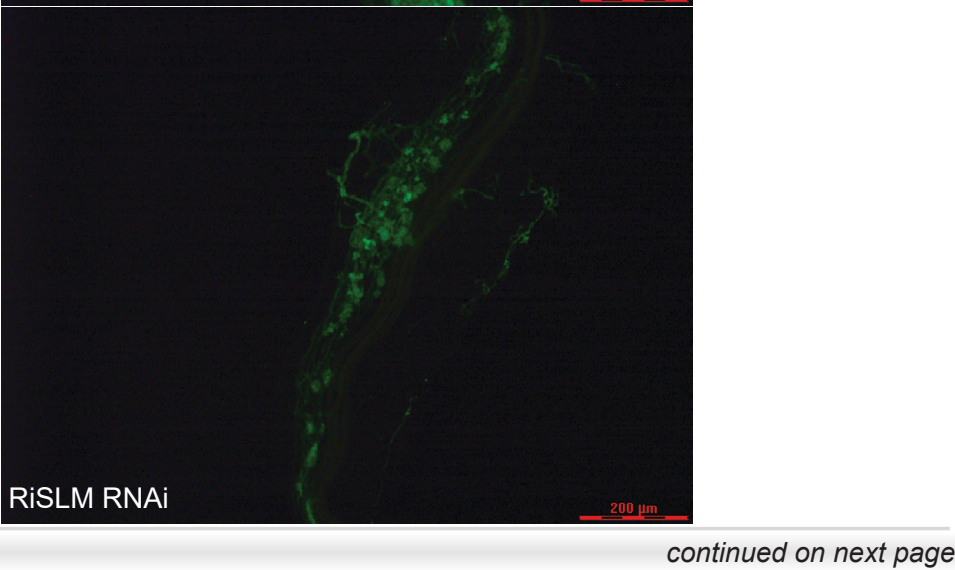


the LysM effectors Mg3LysM from Z $Z$. tritici and Vd2LysM from V. dahliae, which contribute to the virulence of these pathogenic fungi (Marshall et al., 2011; Kombrink et al., 2017). This indicates that LysM effectors are used by both symbiotic and pathogenic fungi to colonize plants (Kombrink and Thomma, 2013).

RiSLM was able to bind both chitin and chitooligosaccharidesas well as to polymeric chitosan, a deacetylated form of chitin, in agreement with Schmitz et al. (2018). Chitin is generally considered to be a more potent trigger of immune responses compared to chitosan in many plants, and several pathogenic fungi deacetylate their chitin as a strategy to evade the immune system (El Gueddari et al., 2002; Rovenich et al., 2016). However chitosan has also been reported to be a strong inducer of immunity in some plants (Sánchez-Vallet et al., 2015). We noticed previously that many chitin deacetylases that are expressed in extraradical mycelium are down-regulated when the fungus grows intraradically in Medicago (Zeng et al., 2018). Therefore, acetylated chitin is likely the most dominant form when $R$. irregularis grows inside the root and where RISLM is most strongly expressed. RiSLM did not bind deactylated $\mathrm{CO} 4$ and $\mathrm{CO} 5$ in microscale thermophoresis, suggesting that RiSLM may only bind to longchain chitosan but not to short-chain deactylated chitooligosaccharides. Several chitinases and chitosanases have been shown to be induced in roots during AM symbiosis (Pozo et al., 1998). Therefore, the protective role of RiSLM to counteract the activity of chitinases may be important to support hyphal growth inside the root. Whether RiSLM forms a protective layer around the fungal cell wall or directly impairs the activity of plant chitinases remains to be demonstrated. This is currently hampered by

continued

Figure 7. Host-induced gene silencing of RiSLM reduces mycorrhization. (a) Frequency $(\mathrm{F} \%)$ remains the same while mycorrhization intensity in the root $(\mathrm{M} \%)$, or arbuscule abundance in the root $(\mathrm{A} \%)$ are reduced in RiSLM silenced roots. For the empty vector control (EV), 12 biological replicates were used. For RiSLM RNAi, 10 biological replicates were used. (b) qPCR analysis of control and RNAi roots showing, RiSLM expression level relative to Rhizophagus EF, RiEF and MtPT4 expression levels relative to Medicago EF (MtEF). Successful silencing of RiSLM (relative to RiEF)reduces RiEF and MtPT4 expression (relative to MtEF), indicating reduced mycorrhization and arbuscule abundance. 12 replicates (individual transgenic roots) were used for EV. 11 replicates were used for RNAi. t-test p-values are indicated for a and b. (c, d) WGA-alexa488 staining of mycorrhization in RiSLM silenced roots (d) or control roots (c). Scale bar $=200 \mu \mathrm{m}$. 


\section{CHAPTER 4}

the fact that biological relevant concentrations of chitinases and their composition in Medicago roots during symbiosis are unknown.

In Medicago chitin-triggered immune responses require perception by the LysM receptor kinases MtLYK9 and MtLYR4, which are thought to form a receptor complex in analogy to the AtCERK1-AtLYK5/4 chitin receptor complex in Arabidopsis (Cao et al., 2014; Bozsoki et al., 2017). The ortholog of MtLYK9 in Lotus japonicus is LjLYS6. Affinity studies showed that the LjLYS6 ectodomain binds CO6-8, with a $K_{\mathrm{d}}$ of $26 \mu \mathrm{M}$ for $\mathrm{CO} 7$ (Bozsoki et al., 2017). This is only slightly lower than the affinity of RiSLM for $\mathrm{CO} 7$, which showed a $K_{\mathrm{d}}$ around $9 \mu \mathrm{M}$. This affinity is significantly lower than the picomolar affinity of the $C$. fulvum LysM effector Ecp6, purified from Pichia pastoris, which contains three LysM domains (de Jonge et al., 2010). Ecp6 was shown to outcompete chitin binding to host receptors in tomato (Sánchez-Vallet et al., 2013). The relatively low affinity of RiSLM for COs raises the question how RiSLM can efficiently interfere with chitintriggered immune responses. Depending on the relative concentration of the effector and receptors it may either sequester COs or, possibly, RiSLM interferes with chitin-induced receptor dimerization/complex formation. However, it should be noted that the in vitro affinity of LysM proteins purified from various heterologous systems can be tricky to compare and may also differ from affinity estimated from biological responses to different ligands. Additional modifications (such as glycosylation) and complex formation with co-receptors may also increase the in planta affinity of the receptors (Chen et al., 2014).

RiSLM was further able to bind a sulphated Myc-LCO as well as CO4, however induction of symbiotic gene expression was not strongly inhibited by the co-application of RiSLM. This is likely due to the higher affinity of the plant receptors for these molecules, although the identity of the receptors in relation to mycorrhization is still elusive. Sulphated Myc-LCOs are structurally very similar to the Nod factors produced by Sinorhizobium meliloti, the rhizobial symbiont of Medicago. S. meliloti Nod factors contain a sulphate group at the terminal reducing sugar are biologically active at picomolar concentrations and are perceived by the LysM receptors MtNFP and MtLYK3 (Dénarié et al., 1996; Arrighi et al., 2006). A S. meliloti nodH mutant, which makes non-sulphated Nod factors, is unable to establish a symbiotic interaction indicating that Medicago has a very high affinity perception system for sulphated LCOs 
(Philippe Roche et al., 1991). This high affinity for sulphated LCO likely explains why RiSLM, despite a higher affinity for sulphated LCO-IV(C18:1, S) (Kd $\sim 15 \mathrm{nM}$ ) than for LCO-IV(C18:1) (Kd $240 \mathrm{nM})$, did not interfere with Myc-sLCOs induced gene expression. Surprisingly, adding equimolar amounts of RiSLM to CO4 appeared to enhance symbiotic signalling (Fig. 5c), suggesting that RiSLM may have an additional role in symbiotic signalling (e.g. interact with Myc factor receptors). However, we cannot fully rule out the possibility that RiSLM enhances symbiotic signalling by binding long chain $\mathrm{CO}$ impurities in the $\mathrm{CO} 4$ preparate.

In conclusion, we revealed an important role for the secreted LysM effector of $R$. irregularis to suppress or evade chitin based immune responses to allow successful colonization of plant roots. RiSLM binding to chitinlike molecules appears to compete with plant LysM receptors for these molecules to favour more symbiotic responses. Intriguingly, we found that also Medicago strongly induces the expression of three secreted single LysM domain proteins (Medtr4g091000, Medtr4g091010 and Medtr4g091020) especially in arbuscule-containing cells, which seem to be conserved in other AM hosts (Gaude, Bortfeld, et al., 2012; Hogekamp and Küster, 2013; Zeng et al., 2018). It will therefore be interesting to investigate whether these putative plant LysM effectors play similar or divergent roles in the intracellular colonization of root inner cortex cells by AM fungi.

\section{Accession numbers}

Genomic or coding sequences of AM secreted LysM proteins (SLMs) mentioned in this research can be retrieved in Genbank under the following accession numbers: RiSLM, XM_025315479; RcSLM, KU305772; RiSLM_A1, MN520639; RiSLM-1_A4, MN520643; RiSLM-2_ A4, MN520642; RiSLM-3_A4, MN520641; RiSLM-4_A4, MN520640; RiSLM_C2, MN520636; RiSLM_B3, MN520637; GmSLM, MN520644; GrSLM, MN520645.

\section{Acknowledgements}

The authors declare no conflict of interest. We would like to thank Natalia Requena for providing the pYST-02 vector. T.B. and E.L. were supported by the European Research Council (ERC-2011-AdG294790). SF and SC acknowledge the technical support of the mass spectrometry 


\section{CHAPTER 4}

and NMR facilities of ICMG (FR2607). JJ.B. acknowledge the Fédération de Recherche Agrobiosciences, Interactions et Biodiversité (FR 3450), CNRS, Université de Toulouse, UPS, Castanet-Tolosan, France, INRA SPE Department and the IDEX "UNITI" Université de Toulouse (GO-AHEAD project) for their contribution to the fundings of the Nanotemper Monolith NT.115.

Supplementary files listed below can be downloaded from the New Phytologist website: https://doi.org/10.1111/nph.16245

Table S1. Primers used in this research.

Table S2. Coding sequences of LysM effectors from different AM fungal species or isolates. 


\section{Supplementary figures}

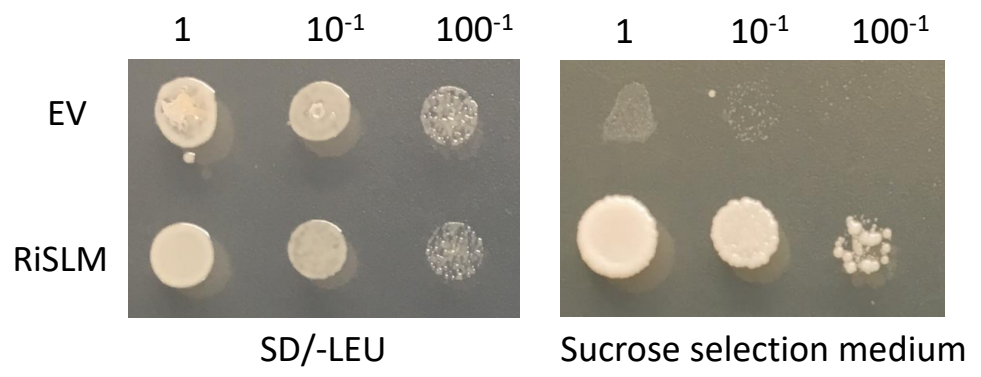

Figure S1. Yeast signal sequence trap. pYST-02-RiSLM, representing a fusion of full length RiSLM (including its endogenous signal sequence) with an invertase, and the empty pYST-02 vector (EV) were transformed into Saccharomyces cerevisiae Y02321, and grown on SD/-Leu (containing glucose) and sucrose selection medium at different dilutions for 3 days at $30^{\circ} \mathrm{C}$.
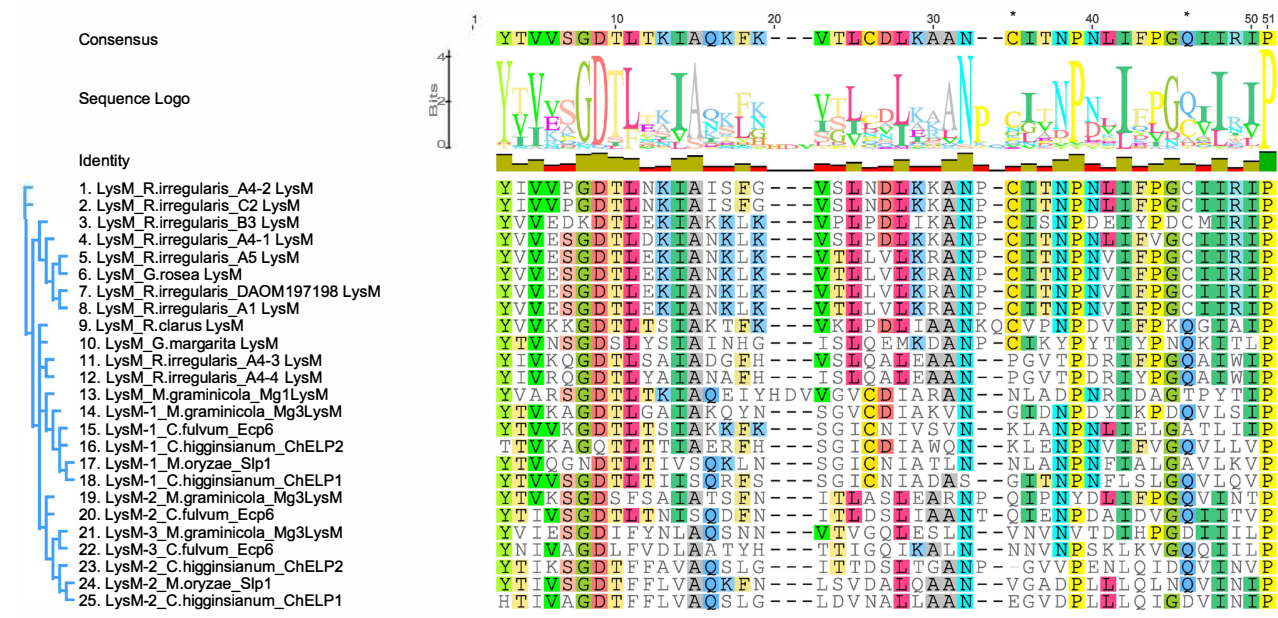

Figure S2. Comparison of LysM domains from AM fungal or pathogenic LysM effectors. MAFFT was used to align all amino acid sequences. Neighbour-joining Tree was built using Geneious tree builder. 


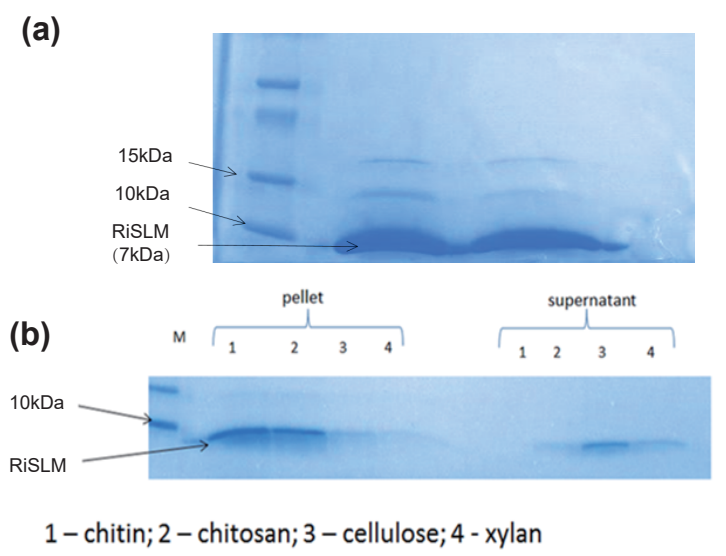

Figure S3. Purified RiSLM protein binds chitin and chitosan. (a) Coomassie stained protein gel of RiSLM (size $\sim 7 \mathrm{kDa}$ ) purified from Escherichia coli ORIGAMI. (b) Coomassie stained protein gel of affinity precipitation assays showing that RiSLM binds to insoluble chitin and chitosan but not xylan and cellulose. For the latter two polymers the vast majority of the protein is retained in the supernatant.

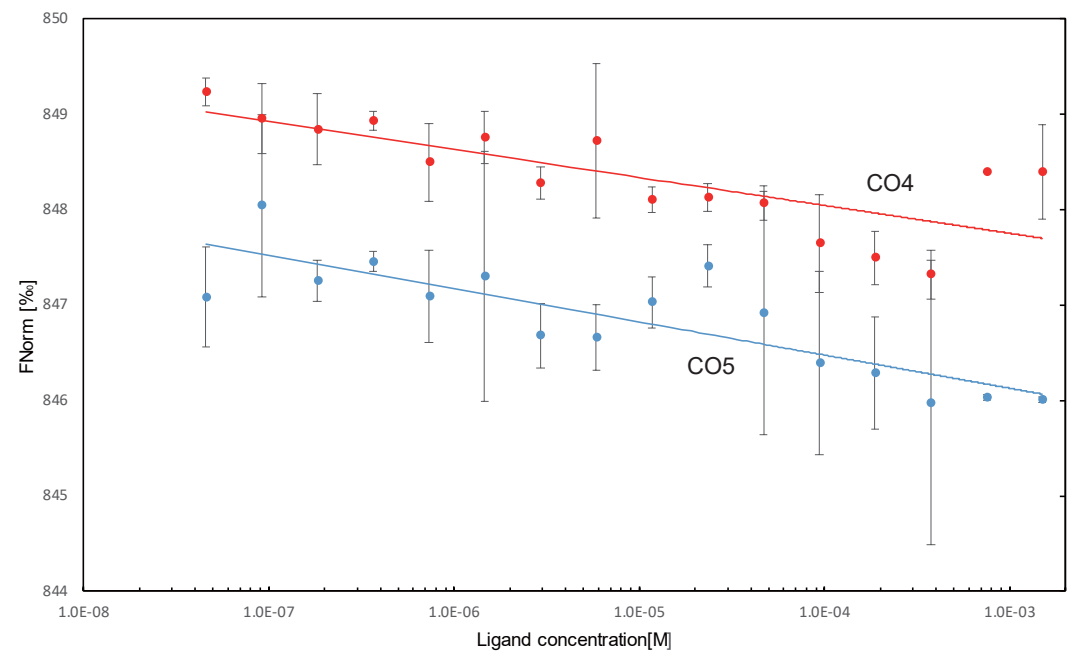

Figure S4. RiSLM does not bind deactylated chitotetraose (CO4) and chitopentaose (CO5) as revealed by microscale thermophoresis. Binding experiments using microscale thermophoresis do not show any interaction with RiSLM in the range of concentrations tested (up to $1 \mathrm{mM}$ ). Error bars represent standard deviation from two independent measurements using two batches of independently purified proteins. 
A LysM effector facilitates AM symbiosis

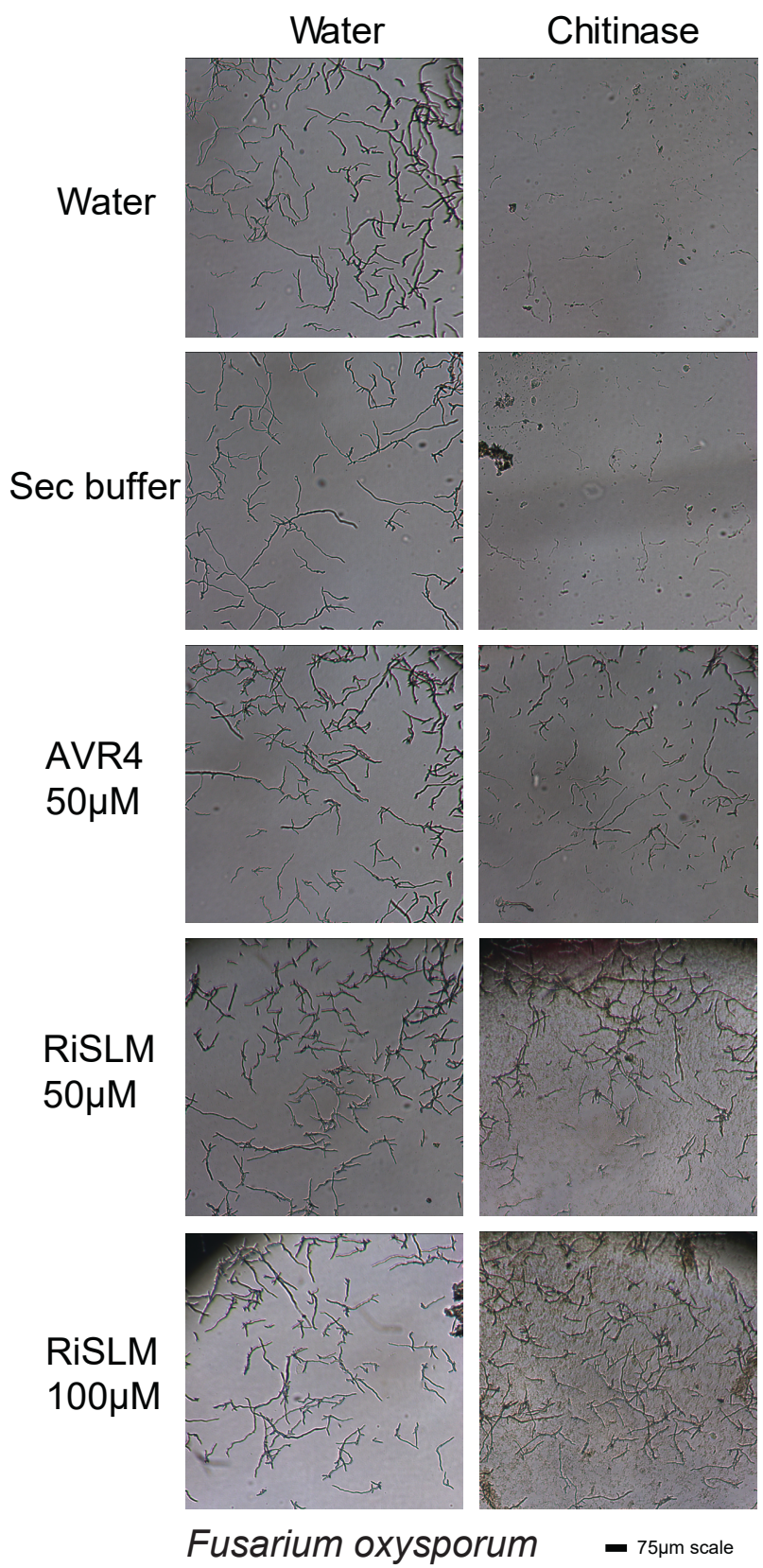

Figure S5. RiSLM protects hyphae from the pathogenic fungus Fusarium oxysporum f.sp lycopersici against plant chitinases. Germinated hyphae are treated with $50 \mu \mathrm{M}$ AVR4, $50 \mu \mathrm{M}$ RiSLM or $100 \mu \mathrm{M}$ RiSLM and incubated at room temperature for 2 hours, and subsequently treated with a tomato chitinase preparation or water for 4 hours. Water or buffer used for size-exclusion chromatography (SEC) were used as negative controls. The Avr4 effector from Cladosporium fulvum was used as positive control. 


\section{CHAPTER 4}

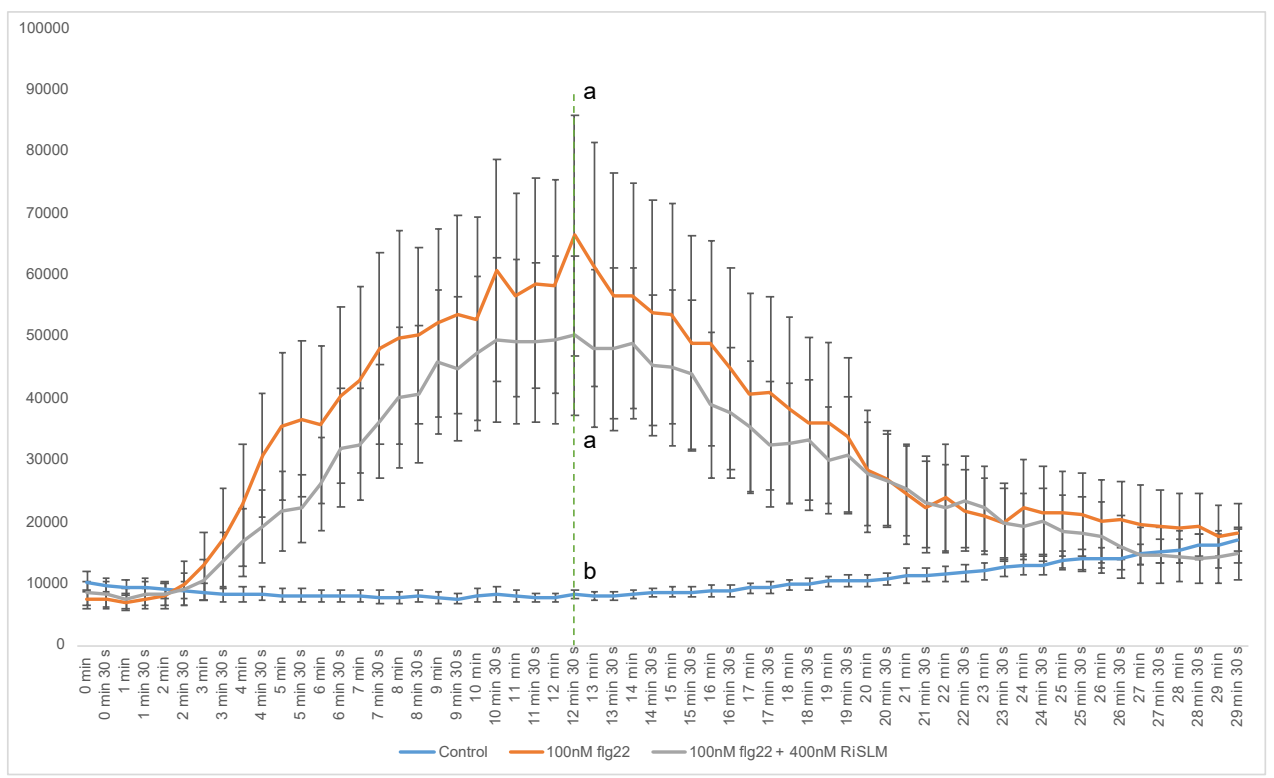

Figure S6. RiSLM does not suppress flg22-induced reactive oxygen species (ROS) production. Medicago truncatula A17 root pieces were treated with $100 \mathrm{nM}$ flg22 with or without 400nM RiSLM . Error bars represent standard error from 6 (control and $100 \mathrm{nM}$ flg22 $+400 \mathrm{nM}$ RiSLM treated) or 5 (100nM flg22 treated) biological replicates. Different letters indicate significant difference (LSD $p<0.05$ ) between different treatments at the indicate time point (green dotted line). 
(a)

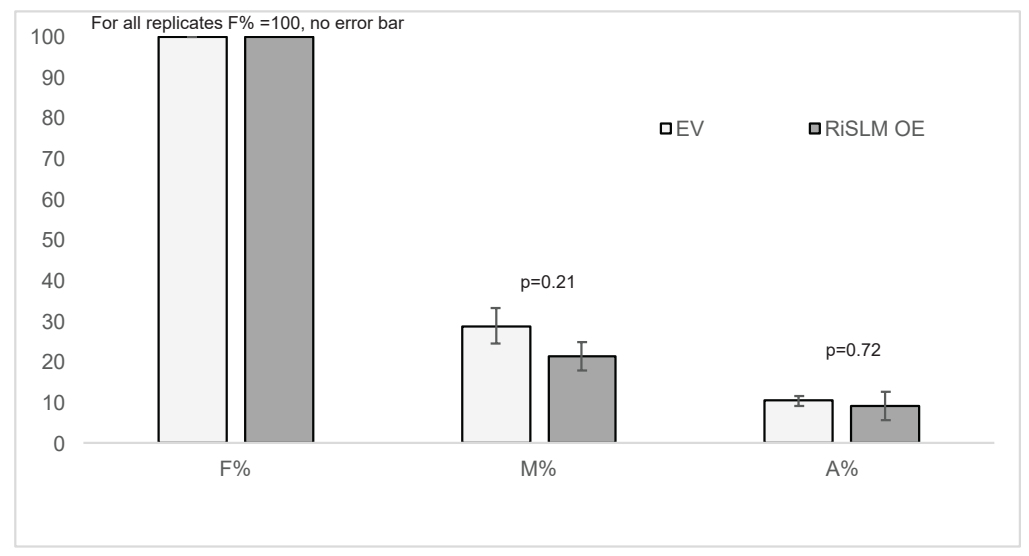

(b)
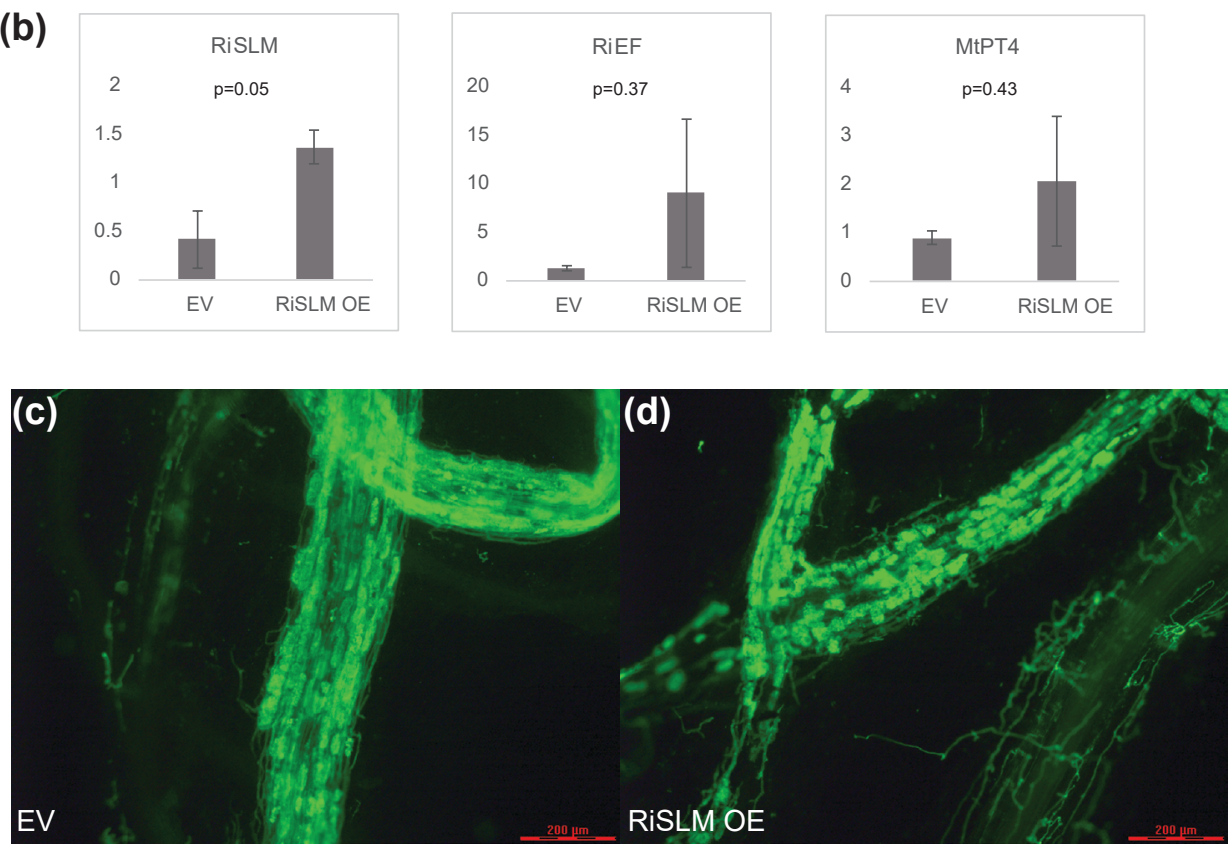

Figure S7. RiSLM overexpression does not enhance mycorrhization in Medicago truncatula. (a) Frequency (F\%), mycorrhization intensity in the root $(\mathrm{M} \%)$, or arbuscule abundance in the root $(\mathrm{A} \%)$ are not affected by overexpressing of RiSLM. (b) qPCR analysis of control and RiSLM overexpressing roots showing RiSLM expression level relative to Rhizophagus irregularis elongation factor RiEF and MtPT4 expression levels relative to Medicago elongation factor MtEF. Error bar represents standard error from 3 replicates. (c, d) WGA-alexa488 staining of mycorrhization in RISLM overexpressed roots (d) or control roots (c). Scale bar = $200 \mu \mathrm{m}$. 


\section{CHAPTER 5}

\section{A Medicago truncatula SWEET transporter implicated in arbuscule maintenance during arbuscular mycorrhizal symbiosis}

Jianyong $A n^{1}$, Tian Zeng ${ }^{2}$, Chuanya $\mathrm{Ji}^{1}$, Sanne de Graaf ${ }^{2}$, Zijun Zheng ${ }^{1}$, Ting Ting Xiao ${ }^{2}$, Xiuxin Deng ${ }^{1}$, Shunyuan Xiao ${ }^{1,3}$, Ton Bisseling ${ }^{2}$, Erik Limpens' ${ }^{2}$, Zhiyong Pan ${ }^{1}$

1. Key Laboratory of Horticultural Plant Biology (Ministry of Education), Key Laboratory of Horticultural Crop Biology and Genetic Improvement (Central Region, Ministry of Agriculture), College of Horticulture and Forestry Sciences, Huazhong Agricultural University, Wuhan 430070, PR China

2. Department of Plant Sciences, Laboratory of Molecular Biology, Wageningen University \& Research, Droevendaalsesteeg 1, 6708 PB Wageningen, Netherlands

3. Institute for Bioscience and Biotechnology Research \& Department of Plant Sciences and Landscape Architecture, University of Maryland College Park, Rockville, MD 20850, USA

Co-corresponding authors:

Zhiyong Pan, zypan@mail.hzau.edu.cn

Erik Limpens, erik.limpens@wur.nl

This chapter has been published in New Phytologist.

https://doi.org/10.1111/nph.15975 


\section{CHAPTER 5}

\section{Abstract}

Plants form a mutualistic symbiosis with arbuscular mycorrhizal (AM) fungi, which facilitates the acquisition of scarce minerals from the soil. In return, the host plants provide sugars and lipids to its fungal partner. However, the mechanism by which the AM fungi obtain sugars from the plant has remained elusive. In this study we investigated the role of potential SWEET family sugar exporters in AM symbiosis in Medicago truncatula. We show that $M$. truncatula SWEET1b transporter is strongly upregulated in arbuscule-containing cells compared to roots and localizes to the peri-arbuscular membrane, across which nutrient exchange takes place. Heterologous expression of MtSWEET $1 b$ in a yeast hexose transport mutant showed that it mainly transports glucose. Overexpression of MtSWEET1b in M. truncatula roots promoted the growth of intraradical mycelium during AM symbiosis. Surprisingly, two independent Mtsweet1b mutants, which are predicted to produce truncated protein variants impaired in glucose transport, exhibited no significant defects in AM symbiosis. However, arbuscule-specific overexpression of MtSWEET1 $\mathrm{b}^{\text {Y57A/ }}$ G58D, which are considered to act in a dominant-negative manner, resulted in enhanced collapse of arbuscules. Taken together, our results reveal a (redundant) role for MtSWEET1b in the transport of glucose across the peri-arbuscular membrane to maintain arbuscules for a healthy mutually beneficial symbiosis.

\section{Introduction}

The vast majority of land plants engage in a mutualistic endosymbiosis with arbuscular mycorrhizal (AM) fungi to acquire mineral nutrients from soil, especially phosphorus and nitrogen (Smith and Smith, 2011). In return, host plants provide organic carbon to AM fungi for their survival and reproduction (Smith and Read, 2008). During the AM symbiosis, hyphae of AM fungi enter root inner cortical cells where they form highly branched hyphal structures, called arbuscules. The arbuscule is surrounded by a specialized host membrane, the peri-arbuscular membrane, which facilitates the exchange of nutrients (Gutjahr and Parniske, 2013). Recently it has been shown that fatty acids are transported from the host to the fungus, likely serving as a major nutritive carbon source (Jiang 


\section{A SWEET transporter implicated in AM symbiosis}

et al., 2017; Luginbuehl et al., 2017; Keymer et al., 2017). However, previously it has been shown that the host also provides sugars as carbon source, although the mechanism by which sugars are transported to the fungus is unclear (Rich et al., 2017; Wang et al., 2017; Roth and Paszkowski, 2017). Therefore, in this study we investigated the role of SWEET sugar transporters.

While it is clear that fatty acids are transported from the host to the AM fungus, several studies have indicated that hexoses, predominantly glucose, are also taken up by the fungus inside the root. For example, isotope-labelled sugars added to mycorrhized roots in combination with detailed nuclear magnetic resonance (NMR) spectroscopic analyses indicated that glucose is directly transferred from the host to the fungus (Shachar-Hill et al., 1995; Solaiman and Saito, 1997; Pfeffer et al., 1999; Bago et al., 2003; Bago et al., 2000). This implies that sugar may be transported via plasma membrane-localized transporters to the apoplast of cortical cells where it is taken up by the intraradical hyphae, or sugar is sent across the peri-arbuscular membrane for uptake by the arbuscule. Consistent with these speculations, a high affinity monosaccharide AM fungal transporter MST2 from Rhizophagus irregularis has been found to be most highly expressed in arbuscules, and this gene is essential for a successful symbiosis (Helber et al., 2011). However, currently no plant sugar transporter has been identified to be involved in sugar transport to the fungus.

In plants the main transporters involved in sugar efflux are members of the SWEET family (S ugar Will Eventually be Exported Iransporter) (Chen et al., 2010; Chen, 2014; Eom et al., 2015). Other sugar transporters, such as sucrose and monosaccharide transporters, are in general functioning in sugar influx/uptake into plants cells (Chen et al., 2015). SWEET transporters are considered to function as sucrose or hexose uniporters, facilitating sugar transport from high to low concentration (Chen et al., 2010; Chen, 2014). Distinct SWEET members have been shown to be involved in different sugar-efflux related processes, such as sucrose loading into the phloem, pollen nutrition by the tapetum or nectar secretion (Chen et al., 2012; Chen, 2014; Lin et al., 2014b). Furthermore, several SWEET encoding genes are transcriptionally induced by biotrophic bacteria or fungi, which actively manipulate the accumulation of SWEET proteins to obtain carbon from the plant (Chen 


\section{CHAPTER 5}

et al., 2010; Chong et al., 2014). In Solanum tuberosum (potato) and Glycine max (soybean), several SWEET transporter genes were found to be induced during mycorrhizal symbiosis (Manck-Götzenberger and Requena, 2016; Zhao et al., 2019). Hence, it has long been anticipated that certain SWEET family members may be recruited to supply sugars to AM fungi.

In this study, we conducted a detailed analysis of the expression pattern of the SWEET family genes in $M$. truncatula roots colonized by AM fungi. We found that MtSWEET1b is highly induced in the cortical cells containing arbuscules of $R$. irregularis. Moreover, MtSWEET1b is specifically localized to the peri-arbuscular membrane. Further genetic analyses suggest that MtSWEET1b may play a redundant role in providing sugar to AM fungi to maintain a successful symbiosis.

\section{Materials and Methods}

\section{Plant materials, growth and mycorrhizal inoculation}

M. truncatula genotypes Jemalong A17 and R108 were used. Additionally, two Tnt1 retrotransposon insertion mutants (NF1309 and NF3539) in the R108 background were used in this study. Tnt1 lines were obtained from the Noble Research Institute (https://medicago-mutant.noble.org/mutant/) (Tadege et al., 2008). The Tnt1 insertion sites were confirmed by transposon display PCR (Tadege et al., 2008), using primers NF1309F/R or NF3539F/R, TntF and TntR (Table S1). All the M. truncatula plants were grown under a $16 \mathrm{~h}$ light and $8 \mathrm{~h}$ dark cycle at $21^{\circ} \mathrm{C}$ and $45 \%$ relative humidity. $R$. irregularis DAOM197198 spores were obtained from Agronutrition, Toulouse, France. The Glomus mosseae strain was purchased from the Institute of Plant Nutrition and Resources, Beijing Academy of Agriculture and Forestry Sciences, and propagated on chives under greenhouse conditions.

M. truncatula mutant seedlings or Agrobacterium rhizogenes transformed composite $M$. truncatula plants were grown in cones (Stuewe \& Sons, USA) containing a 1.5:1 ( $\mathrm{v} / \mathrm{v}$ ) ratio of autoclaved 2-5 mm clay granules (Jongkind, The Netherlands) and sand mixture. Each cone contained one plant, 400 commercial $R$. irregularis spores were placed on a sand 
layer positioned about $7 \mathrm{~cm}$ below the top of the cones. Plants were watered twice a week with $10 \mathrm{ml}$ low-phosphate $(20 \mu \mathrm{M} \mathrm{Pi})$ half-strength Hoagland's solution.

\section{Vector construction and hairy root transformation}

To create MtSWEET1bpro:GUS, a 1349 bp upstream of the MtSWEET1b (Medtr3g089125) ATG start codon was amplified by PCR, from $M$. truncatula A17 genomic DNA, using high fidelity Phusion (Thermal Fisher) polymerase with primers pSWEET1b-F and pSWEET1b-R and was then cloned into $p E N T R-D /-T O P O$ (Invitrogen). After sequence verification, the MtSWEET1b promoter region was recombined into pKGWFS2-RR using LR clonase II (Invitrogen), resulting in MtSWEET1bpro:GUS. Primer sequences used in this study are listed in Table S1.

The A17 genomic sequence of MtSWEET1b lacking the stop codon was PCR amplified using primers MtSWEET1b-F and SWEET1b-R and then cloned into pENTR-D/-TOPO-vector. The MtSWEET1b promoter was digested from pENTR-D/-TOPO (HindIII -AscI) and inserted into a pENTR4-1 (BsaI digested) entry vector using T4 DNA Ligase. MtSWEET1bpro:MtSWEET1b-GFP was created using multisite Gateway technology (Invitrogen) in the pKGW-RR-MGW destination vector, which contains a DsRed selection marker to select transgenic roots (Huisman et al., 2016). To create an overexpression construct of MtSWEET1b, the genomic sequence of MtSWEET1b in PENTR-D/-TOPO-vector was used to incorporate MtPT4pro:MtSWEET1b in the pKGW-RR-MGW destination vector via multisite gateway technology.

Y57A-MtSWEET1b or G58D-MtSWEET1b substitutions (with or without stop-codon) were created by overlap PCR using wild type MtSWEET1b cDNA as a template and subsequently cloned into pENTR-D/-TOPO vector. Multisite gateway technology was subsequently used to create MtPT4pro:Y57A-MtSWEET1b-GFP, MtPT4pro: G58D-MtSWEET1b-GFP, MtPT4pro:Y57A-MtSWEET1b and MtPT4pro: G58D-MtSWEET1b in the pKGW-RR-MGW destination vector according to a previous method (Huisman et al., 2016). All constructs were confirmed by Sanger sequencing.

A. rhizogenes strain MSU440 was used for hairy root transformations according to previously described (Limpens et al., 2004). 


\section{CHAPTER 5}

\section{Mycorrhizal staining and quantification}

Roots were harvested and fixed in 50\% ethanol or PME (100 mM PIPS, $1 \mathrm{mM} \mathrm{MgCl}{ }_{2}, 1 \mathrm{mM}$ EGTA, pH 6.65) buffer for staining. To visualize mycorrhizal structures, roots were stained using wheat germ agglutinin (WGA) Alexa Fluor 488 . Roots were incubated at $90^{\circ} \mathrm{C}$ for $10 \mathrm{~min}$ in $10 \%$ $\mathrm{KOH}$, washed twice in water, washed in $2 \% \mathrm{HCl}$ for 20 min, washed in $1 \mathrm{x}$ PBS buffer for $1 \mathrm{~h}$, and then incubated overnight in 1x PBS containing 2 $\mathrm{mg} / \mathrm{L}$ WGA-Alexa Fluor 488 at $4^{\circ} \mathrm{C}$.

Quantification of mycorrhization was done according to Trouvelot et al. (1986) or the objective intersect method (McGONIGLE et al., 1990). Arbuscule morphology was scored into two categories, the abundance of good/mature arbuscules and degenerating/collapsed arbuscules was calculated separately.

\section{Laser microdissection}

M. truncatula roots colonized by $R$. irregularis were harvested and fixed in Farmer's fixative solution and stored at $4^{\circ} \mathrm{C}$ for $16 \mathrm{~h}$. To isolate the arbuscule-containing cells and non-colonized inner cortical cells, the roots were stained by $0.01 \%$ chlorazol black $E$, dehydrated using an ethanol series, and embedded using Steedman wax (Sigma). Laser microdissection was performed according to Huisman et al. (2016) using a Leica LMD7000. RNA was isolated from microdissected samples (three biological replicates each) using the Qiagen RNeasy microkit. Quality of the isolated RNA was monitored using agarose gel electrophoresis and by Agilent Technologies 2100 Bioanalyzer. The cDNA synthesis and cDNA pre-amplification before qRT-PCR analysis were performed as described by Huisman et al. (2016).

\section{RNA extraction and quantitative real-time PCR (qRT-PCR) analysis}

Total RNA from roots was isolated using the Qiagen Plant RNeasy mini kit, according to manufacturer's instructions. Quality of the isolated RNA was monitored using agarose gel electrophoresis. A total of $500 \mathrm{ng}$ or 1 $\mu g$ RNA was used to synthesize cDNA using the iScript cDNA synthesis kit (Bio-Rad). Resulting CDNA was diluted 5 times and used for qRT-PCR analysis using SYBR green supermix (Bio-Rad) with gene specific primers (listed in Table S1) in a Bio-Rad CFX connect real-time PCR machine. The 
cDNA levels were normalized using the widely used housekeeping gene Medicago elongation factor 1alpha MtEF1a as a reference gene.

\section{RNAseq analyses}

The raw RNA-sequencing data of cortical cells containing arbuscules and cortical cells adjacent to hyphae were obtained by Zeng et al. (2018). All the clean reads were mapped to the $M$. truncatula genome version Mt 4.0 (Tang et al., 2014) using CLC genomics workbench 10.0.1 (Qiagen). The parameters used for mapping and TPM (transcripts per million, Wagner et al., 2012) calculation are described in Zeng et al. (2018).

\section{Yeast expression of MtSWEET1b and its dominant negative mutants}

MtSWEET1b and AtSWEET1 ORFs were PCR amplified on CDNA from M. truncatula $A 17$ and Arabidopsis Col-0, respectively, using primers containing NotI sites and BsaI sites, and inserted into the yeast expression vector pPMA1 (Sauer and Stolz, 1994). The final MtSWEET1b-pPMA1 and AtSWEET1-pPMA1 constructs were verified by Sanger sequencing. MtSWEET1b-G57A and MtSWEET1b-G58D and wild type MtSWEET1b ORF pENTR clones were recombined into the pDR-F1-GW vector (Xuan et al., 2013) using LR clonase II (Invitrogen ${ }^{\mathrm{TM}}$ ). Also the truncated transcript of MtSWEET1b produced in sweet1b-2 mutant (NF3539) was cloned from its CDNA, and recombined into the pDR-F1-GW vector as above. All vectors were introduced into the yeast hexose transporter mutant EBY. VW4000 (Wieczorke et al., 1999) via PEG/LiAc mediated transformation (Gietz and Schiestl, 2007). After transformation, yeast transformants were incubated on selective dropout (SD, -URA) medium containing $2 \%$ maltose (Dicofa) as a carbon source for $2-3$ days at $30^{\circ} \mathrm{C}$. Presence of the construct in the yeast transformants was verified by plasmid isolation and resequencing. For complementation growth assays, the transformants were grown in YNB liquid medium containing $2 \%$ maltose overnight, washed twice in sterile water and resuspended at an OD600 of 0.2. Subsequently, serial dilutions ( $1 \mathrm{x}, 5 \mathrm{x}, 25 \mathrm{x}$ and $125 \mathrm{x}$ ) were plated on SD (-URA) media containing either $2 \%$ maltose as control or $2 \%$ glucose, $2 \%$ fructose, $2 \%$ mannose, $2 \%$ galactose and $2 \%$ sucrose, respectively. Growth was recorded after $2-4$ days at $30^{\circ} \mathrm{C}$.

Phylogenetic analyses and transmembrane helices prediction 


\section{CHAPTER 5}

M. truncatula, Lotus japonicus and S. tuberosum SWEET homologs were identified from their genome databases with BLASTP using Arabidopsis and rice SWEET proteins as queries. All the protein sequences were aligned by ClustalW using MEGA 6.06 version (Tamura et al., 2013). A phylogenetic tree was constructed using the Neighbor-joining method with 2000 bootstrap replications and 95\% Gaps/Missing data deletion. Figtree v1.4.2 was used to adjust shape of the phylogenetic tree.

The transmembrane domain predication of MtSWEET1b and its truncated versions were conducted by TMHMM Server v 2.0 (http://www.cbs.dtu. dk/services/TMHMM/) online software.

\section{GUS staining, resin embedding and sectioning}

Transgenic roots containing MtSWEET1bpro:GUS constructs were stained by GUS buffer. Therefore roots were harvested and washed with $1 \mathrm{x}$ PBS twice, incubated in $\beta$-glucoronidase (GUS) reaction buffer ( $3 \%$ sucrose, $10 \mathrm{mM}$ EDTA, $2 \mathrm{mM}$ potassium-ferrocyanide, $2 \mathrm{mM}$ potassiumferricyanide and $1 \mathrm{mg} / \mathrm{ml} \mathrm{X-Gluc} \mathrm{in} 100 \mathrm{mM}$ PBS, $\mathrm{pH}$ 7.0) under vacuum for $30 \mathrm{~min}$, and then incubated at $37^{\circ} \mathrm{C}$ for $3 \mathrm{~h}$. After GUS staining, the root segments were incubated in fixation buffer $(5 \%$ glutaraldehyde in $100 \mathrm{mM}$ phosphate buffer, $\mathrm{pH} 7.2$ ) at $4^{\circ} \mathrm{C}$ overnight. Next, the roots were dehydrated using an ethanol series, infiltrated and embedded in Technovit 7100 (Hereus-Kulzer, Germany). The embedded roots were cut into $10 \mu \mathrm{m}$ longitudinal sections using a microtome (Leica 2035) and stained in $0.1 \%$ Ruthenium Red for $15 \mathrm{~min}$.

\section{Microscopy}

Transgenic roots showing DsRed fluorescence were selected using a Leica MZIII fluorescence stereomicroscope. To observe the subcellular localization of GFP-fusion constructs, a Leica SP8 confocal microscope was used. Freshly harvested transgenic roots were dissected longitudinally using a razorblade, mounted on microscope slides in water, and imaged using a 40x water-immersion objective. GFP was observed using an excitation wavelength of $488 \mathrm{~nm}$, and emission detected between 500 and $550 \mathrm{~nm}$. DsRed was observed using excitation wavelength of 554 $\mathrm{nm}$, and emission detected at $586 \mathrm{~nm}$. For imaging of GUS-stained root sections or WGA-Alexa Fluor 488 stained roots, a Leica DM5500B fluorescence microscope was used. 


\section{Results}

\section{MtSWEET1b is strongly expressed in arbuscule-containing cells}

To investigate whether host SWEET transporters are engaged in sugar transport during mycorrhizal symbiosis, we first identified M. truncatula genes encoding SWEET transporters based on their sequence homology with Arabidopsis thaliana and Oryza sativa SWEET proteins (Chen et al., 2010). A total of 26 M. truncatula SWEET genes were identified in the M. truncatula genome (Table S2), in agreement with previously reported (Kryvoruchko et al., 2016). The phylogenetic relationship of the $26 \mathrm{M}$. truncatula SWEET genes and their $A$. thaliana, O. sativa, S. tuberosum and $L$. japonicus homologs is shown in Fig. S1.

To identify which SWEET genes may be involved in AM symbiosis, we determined the expression patterns of all SWEET genes in M. truncatula roots inoculated with the AM fungus $R$. irregularis. First, we analyzed RNAseq transcriptome data of cortical cells containing arbuscules and cortical cells adjacent to hyphae, which were isolated by laser microdissection (Zeng et al., 2018). This analysis showed that of all the SWEET genes only MtSWEET1b (Medtr3g089125) has high expression in root cells that are in contact with the fungus (Fig. S2). MtSWEET1b is especially prominent in arbuscule-containing cells, with a TPM value (transcripts per million) of $326.32 \pm 61.40$. In comparison, the highly expressed phosphate transporter MtPT4 (Harrison et al., 2002; Javot et al., 2007) has a TPM value of $1399.87 \pm 385.28$. We confirmed that MtSWEET1b is highly expressed in the cortical cells containing arbuscules by laser capture microdissection combined with qRT-PCR analysis (Fig. S3a). Five additional SWEET transporters that appeared to be weakly expressed based on RNA-seq data were hardly detectable by qRT-PCR (Fig. S2 and S3a). Low MtSWEET1b expression (TPM value $46.00 \pm 47.26$ ) was also detected in cortical cells surrounding intercellular hyphae (Fig. S2). qRT-PCR analysis showed that the expression level of MtSWEET1b is 200-fold higher in mycorrhized roots one month after inoculation with $R$. irregularis compared to non-mycorrhized roots (Fig. 1a). Induction of MtSWEET1b expression upon AM symbiosis was also observed in roots colonized by the AM fungus $F$. mosseae (Fig. S3b). Interestingly, MtSWEET1b expression was rapidly shut down when the concentration of supplied phosphate was raised from $20 \mu \mathrm{M}$ to $3 \mathrm{mM}$ (Fig. S4a). It has 


\section{CHAPTER 5}

been shown that high concentrations of phosphate repressed symbiotic gene expression, leading to an inhibition of colonization with a lag period of more than one week in established mycorrhizal roots (Breuillin et al., 2010; Helber et al., 2011). Three days after increasing the phosphate concentration, a time point when arbuscule abundance and morphology were not affected, expression of MtSWEET1b as well as the symbiotic marker genes MtPT4 and MtBCP1 were significantly down-regulated (Fig. $\mathrm{S} 4 \mathrm{~b})$. This indicates that transcriptional regulation of MtSWEET1b occurs in a phosphate concentration-dependent manner.

To further study the spatial expression pattern of MtSWEET1b, we fused the putative 1349 bp promoter region upstream of the MtSWEET1b start codon to the open reading frame of the $\beta$-glucoronidase (GUS) reporter. We introduced this promoter-GUS construct into roots of $M$. truncatula A17 by $A$. rhizogenes-mediated root transformation and assayed for GUS activity in transgenic roots inoculated with or without $R$. irregularis, at 4 weeks post-inoculation (wpi). Analysis of semi-thin longitudinal root sections showed strong GUS signal present in arbuscule-containing cells

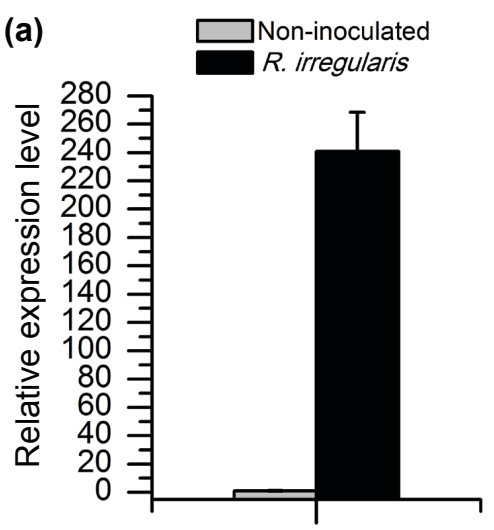

MISWEET1b

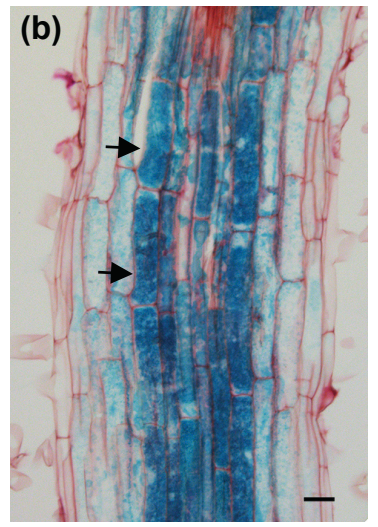

$R$. irregularis

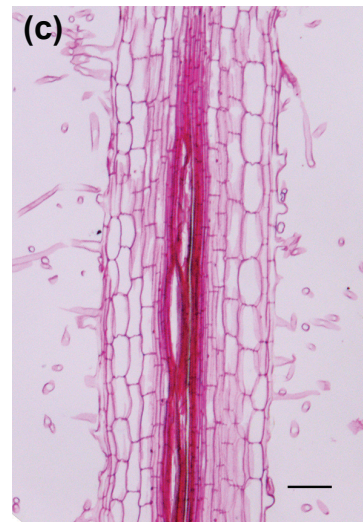

Non-inoculated

Figure 1. Analysis of the expression pattern of $M$. truncatula SWEET1b during AM symbiosis. (a) Relative expression level of MtSWEET1b in M. truncatula roots inoculated with AM fungi $R$. irregularis and non-inoculated controls measured by qRT-PCR. Mycorrhized and non-inoculated control roots were harvested at 6 wpi. $M t E F 1 a$ was used as reference gene. SD obtained from three independent replicates. (b) Medicago hairy roots expressing MtSWEET1bpro:GUS show GUS activity in arbuscule-containing cells (arrow) and weak signal in the surrounding cortex cells, 6 wpi with $R$. irregularis. Bar $=25 \mu \mathrm{m}$. (c) Non-mycorrhized MtSWEET1bpro:GUS expressing roots lack GUS signal in the cortex cells in the mature part of the root. Bar $=75 \mu \mathrm{m}$. 
(Fig. 1b, indicated by arrow) and weakly in the surrounding cortical cells (Fig. 1b). Additionally, GUS signal was also detected in the root tips of both inoculated and non-inoculated roots (Fig. S5a-b). No GUS signal was observed in cortical cells of non-mycorrhized roots (Fig. 1C).

These data suggest that MtSWEET1b is a good candidate gene for a role in sugar transport in arbuscule-containing cells.

\section{MtSWEET1b localizes to the peri-arbuscular membrane}

To determine the subcellular localization of MtSWEET1b, we made a translational fusion of MtSWEET1b with GFP driven by its own 1349-bp promoter, creating MtSWEET1b:MtSWEET1b-GFP for protein localization analysis in transgenic $M$. truncatula roots inoculated with $R$. irregularis. Confocal imaging showed that MtSWEET1b-GFP is specifically localized to the peri-arbuscular membrane surrounding both fine hyphal branches and the trunk of the arbuscule (Fig. 2a-e). This result suggests that MtSWEET1b is well positioned to transport sugars across the periarbuscular membrane to the fungus.
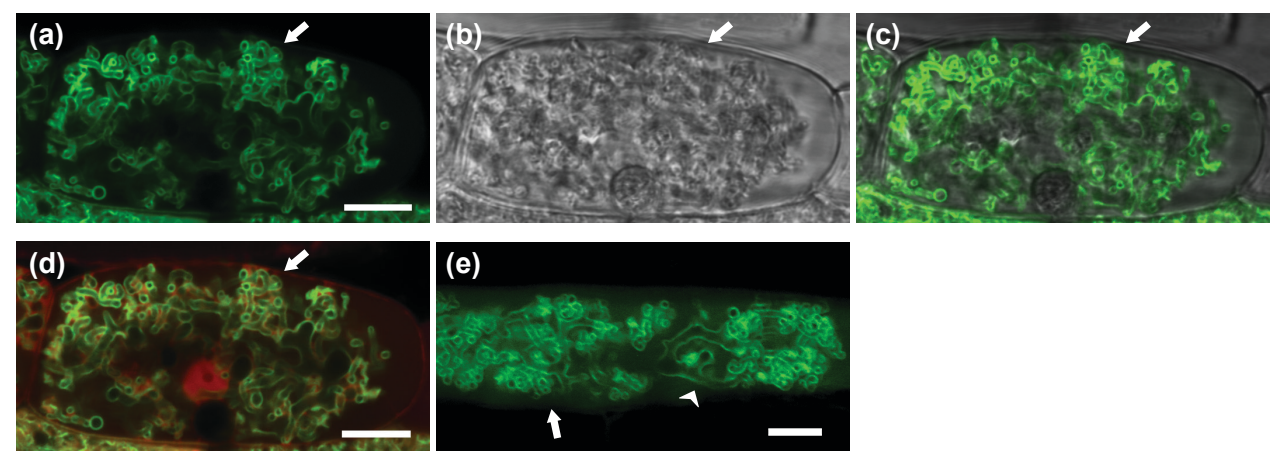

Figure 2. MtSWEET1b-GFP is present on the peri-arbuscular membrane. (a-e) Confocal microscopy images of $M$. truncatula roots expressing MtSWEET1bpro:MtSWEET1b-GFP colonized with $R$. irregularis. (a-d) Different panels display the MtSWEET1b-GFP signal in a single cortical cell harboring a mature arbuscule. (a) GFP-fluorescence of MtSWEET1b-GFP can be observed on the peri-arbuscular membrane at fine branches (arrow). (b) Corresponding bright field image. (c) Corresponding overlay image of (a) and (b). (d) Corresponding overlay image of green channels (a) and red channels. Red fluorescence in the cytoplasm and nucleus was from the DsRed protein expressed from the A. thaliana Ubiquitin3 promoter. (e) A cortical cell harboring a developing arbuscule displays SWEET1b-GFP signal on the peri-arbuscular membrane at fine branches (arrow) as well as the trunk part (arrowhead). All bars $=10 \mu \mathrm{m}$. 


\section{CHAPTER 5}

\section{MtSWEET1b is a glucose transporter}

MtSWEET1b belongs to the AtSWEET1 clade (Fig. S1, see also Kryvoruchko et al., 2016). Arabidopsis AtSWEET1 has previously been identified as a glucose transporter that is mainly expressed in flowers (Chen et al., 2010). Arabidopsis has only a single gene in this clade, while the AM-host plants M. truncatula, O. sativa, L. japonicus and S. tuberosum have at least two genes in this clade. In M. truncatula, only MtSWEET1b is strongly upregulated in arbuscule-containing cells compared to roots, while the other homolog (MtSWEET1a, Medtr1g029380) is mainly expressed in the flower, in analogy to AtSWEET1, based on the Medicago Gene Expression Atlas (Benedito et al., 2008). Also, in L. japonicus and S. tuberosum, it has been shown that one of the SWEET genes (Lj0g3v0035419.1 and StSWEET1b, respectively) of this clade is induced upon mycorrhization (Handa et al., 2015; Manck-Götzenberger and Requena, 2016; Sugimura and Saito, 2017).

The phylogenetic relation of MtSWEET1b to AtSWEET1 suggested that it may be a glucose transporter (Fig. S1). To determine the sugar transport ability and specificity of MtSWEET1b, we expressed MtSWEET1b in the hexose transport-deficient $S$. cerevisiae mutant EBY.VW4000. In this mutant yeast line all 20 endogenous sugar transporter genes are knocked-out by which it is unable to grow on hexoses or sucrose, but it can still grow on the disaccharide maltose (Wieczorke et al., 1999). The full-length MtSWEET1b cDNA was cloned into the yeast pPMA1 expression vector, and as a positive control AtSWEET1 CDNA was also cloned into the PPMA1 vector (Sauer and Stolz, 1994). After transformation into the EBY. VW4000 yeast strain, we investigated its growth ability on selection plates containing D-glucose, D-fructose, D-mannose, D-galactose or D-sucrose, respectively. This showed that MtSWEET1b, like AtSWEET1, enables the yeast mutant EBY.VW4000 to grow well on glucose, but not fructose, mannose, galactose or sucrose (Fig. 3), indicating that MtSWEET1b can function as a glucose transporter.

\section{Overexpression of MTSWEET1 $\mathrm{b}$ promotes the growth of intraradical} AM mycelium

Peri-arbuscular membrane specific localization of MtSWEET1b suggests that it may function at the peri-arbuscular membrane to transport sugar 

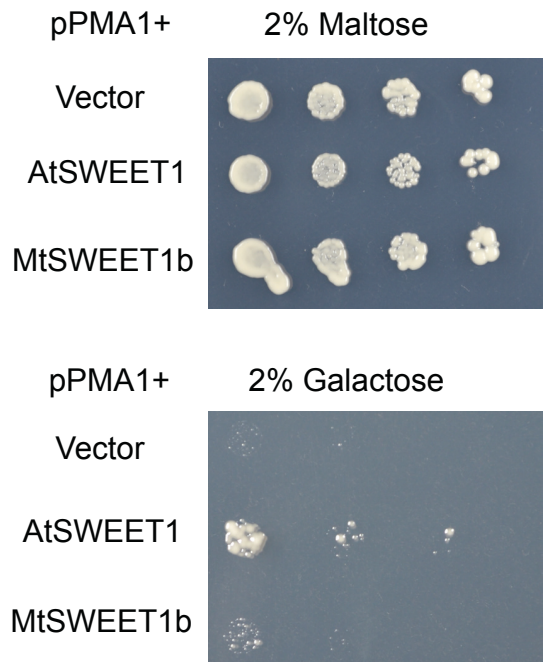

2\% Glucose

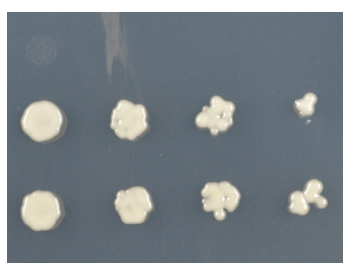

2\% Mannose

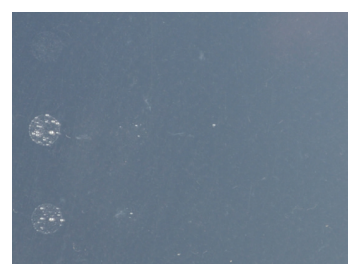

$2 \%$ Fructose

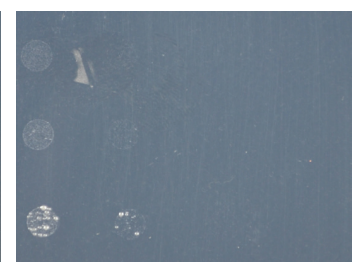

$2 \%$ Sucrose

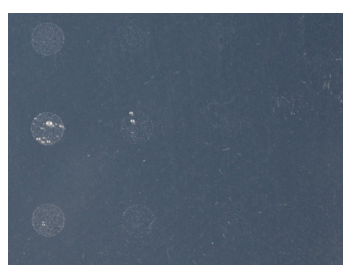

Figure 3. Complementation of the yeast hexose transport defective strain EBY.VW4000 by MtSWEET1b. The yeast hexose transport defective strain EBY. VW4000 was transformed with the pPMA1 empty vector, pPMA1-AtSWEET1 and pPMA1-MISWEET1b, respectively. The images show the growth of empty vector, MtSWEET1b or AtSWEET1 transformed EBY.VW4000 on SD media containing different sugars as sole carbon source at $30^{\circ} \mathrm{C}$ for 3 days. EBY.VW4000 can only grow on SD media containing maltose, but not on media containing other hexoses or sucrose. Empty vector and AtSWEET1 transformed yeast were used as negative and positive control, respectively. In each image, the yeast strain was diluted to an OD600 of 0.2 and then $1 \times, 5 \times, 25 \times, 125 \times$ diluted by sterile water.

(glucose) to AM fungi to fuel fungal growth. To test this speculation, we overexpressed MtSWEET1b using the arbuscule-specific MtPT4 promoter in M. truncatula hairy roots, and then measured AM colonization level in MtSWEET1b overexpression lines (OE-MtSWEET1b) and empty vector transformed lines (EV control) 5 wpi with $R$. irregularis. qRT-PCR confirmed that the expression level of MtSWEET1b was indeed significantly increased in OE-MtSWEET1b compared with EV controls (Fig. S6a). We found that, although the intensity of mycorrhization in infected roots (m\%) (Fig. 4a) and arbuscule abundance (Fig. S6b-c) were not affected, the frequency $(F \%, p<0.05)$ and intensity of mycorrhization $(M \%, p=$ $0.1047)$ in the whole roots were increased or showed the tendency to be increased in OE-MtSWEET1b compared with EV control (Fig. 4a). In line with this, the expression level of $R$. irregularis housekeeping gene RiEF also showed $\sim 50 \%$ higher expression in OE-MtSWEET1b roots compared with EV control roots (Fig. 4b). Similar results were obtained in a second 


\section{CHAPTER 5}

independent replicate experiment (Fig. S7). These results indicate that MtSWEET1b can enhance the intraradical growth of AM fungi during the symbiosis.

\section{Glucose transport activity of MtSWEET1b seems dispensable for mycorrhization}

To further identify the function of MtSWEET1b in AM symbiosis, we isolated two Tnt1-transposon insertion lines, NF1309 (named sweet1b-1) and NF3539 (named sweet1b-2), from the M. truncatula Tnt1 collection using a nested-PCR approach (Tadege et al., 2008). The sweet $1 b-1$ and sweet $1 b-2$ mutants contain a Tnt1 insertion in exon 3 and exon 4, respectively (Fig. 5a). Homozygous sweet $1 b-1$ and sweet $1 b-2$ lines

(a)

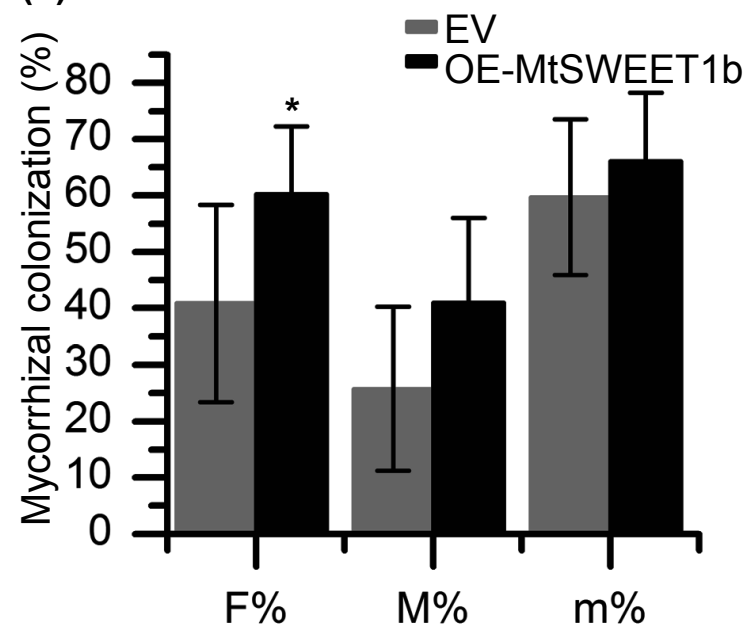

(b)

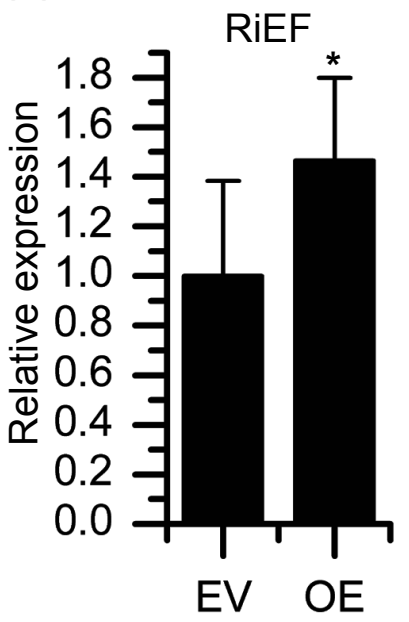

Figure 4. Overexpression of MtSWEET1b increased mycorrhization levels in M. truncatula roots.

(a) Quantification of mycorrhization level in $M$. truncatula roots expressing MtPT4pro:MtSWEET1b $(\mathrm{n}=6)$ and empty vector as control $(\mathrm{n}=6) 5$ wpi with $R$. irregularis. The parameters are according to Trouvelot et al. (1986): $\mathrm{F} \%$, the frequency of analyzed root fragments that are mycorrhized; $\mathrm{M} \%$, the intensity of infection in the whole roots; $\mathrm{m} \%$, the intensity of infection in mycorrhized root fragments. EV, empty vector lines. OE-MtSWEET1b, overexpression MtSWEET1b lines. Data are represented as the mean $\pm S D$ of six biological replicates. ${ }^{*} P<0.05$, Student's t-test. (b) qRT-PCR measurement of the expression level of RiEF in $M$. truncatula roots expressing MtPT4pro:MtSWEET1b $(n=6)$ and empty vector as control $(n=6)$ at 5 wpi with $R$. irregularis. $E V$, empty vector lines. OE, overexpression $M t S W E E T 1 b$ lines. MtEF1 $\alpha$ was used as reference gene. Data are represented as the mean $\pm S D$ of six biological replicates. ${ }^{*} P<0.05$, Student's t-test. 
were identified, and monitored for mycorrhization levels using the objective intersect method (McGONIGLE et al., 1990). Unexpectedly, at 6 wpi the insertion lines (sweet1b-1 and sweet1b-2) did not show any difference in mycorrhizal colonization levels as compared with the wildtype plants (Fig. 5b). Because MtSWEET1b expression is upregulated in arbuscule-containing cells, we further investigated arbuscule morphology by classifying the arbuscules into good/mature arbuscules (Fig. 6a, two left panels) and degenerating/collapsed arbuscules (Fig. 6a, two right panels). However, no difference in the abundance of these classes was observed (Fig. 5c).

(a)

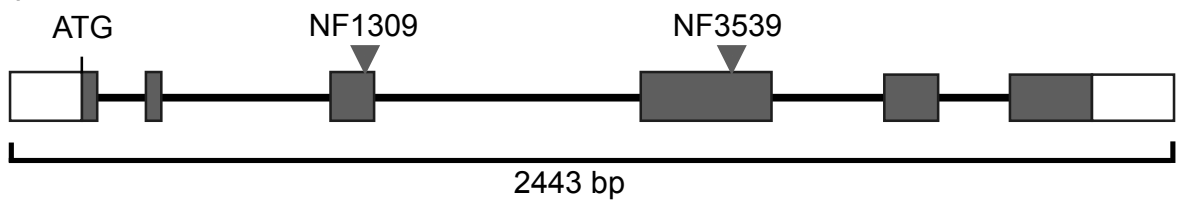

(b)

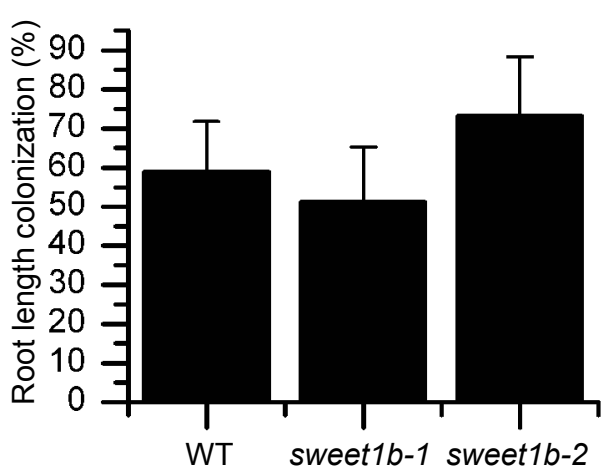

(c)

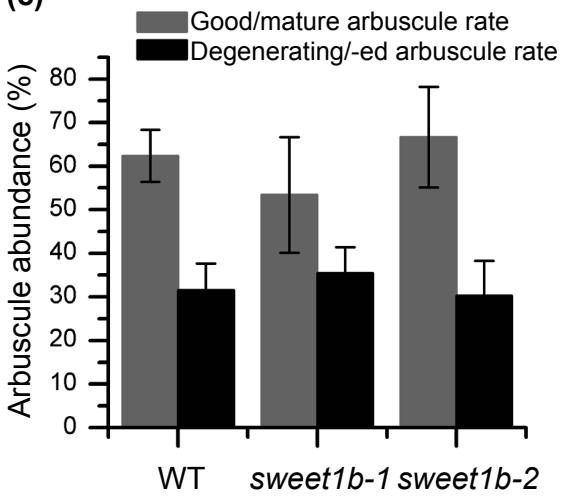

Figure 5. Two independent Mtsweet1b Tnt1 insertion mutants do not show defects in mycorrhization. (a) The gene structure of MtSWEET1b and the Tnt1 transposon insertion positions of two independent mutants. The gray boxes indicate 6 exons. In NF1309 (sweet1b-1) and NF3539 (sweet1b-2), the Tnt1 insertion is located in exon 3 and exon 4, respectively. (b) Level of root length colonization of the two mutants and wild type R108 5 wpi with $R$. irregularis. No difference was detected in root length colonization between control $(\mathrm{n}=5)$ and sweet1b-1 $(\mathrm{n}=$ $9, P=0.2402)$ or sweet $1 b-2(n=8, P=0.1065)$ using Student's t-test. (c) Good/ mature and degenerating/degenerated arbuscule abundance in wild type R108 (WT $\mathrm{R} 108, \mathrm{n}=5$ ) and two independent mutants 5 wpi with $R$. irregularis. No difference was observed in arbuscule abundance between control $(n=5)$ and sweet $1 b-1(n=$ 8 , Good arbuscule $P=0.1837$; Degenerating arbuscule $P=0.2812$ ) or sweet $1 b-2$ $(n=8$, Good arbuscule $P=0.4622$; Degenerating arbuscule $P=0.7810$ ) based on Student's t-test. Error bars indicate SD. 
(a)
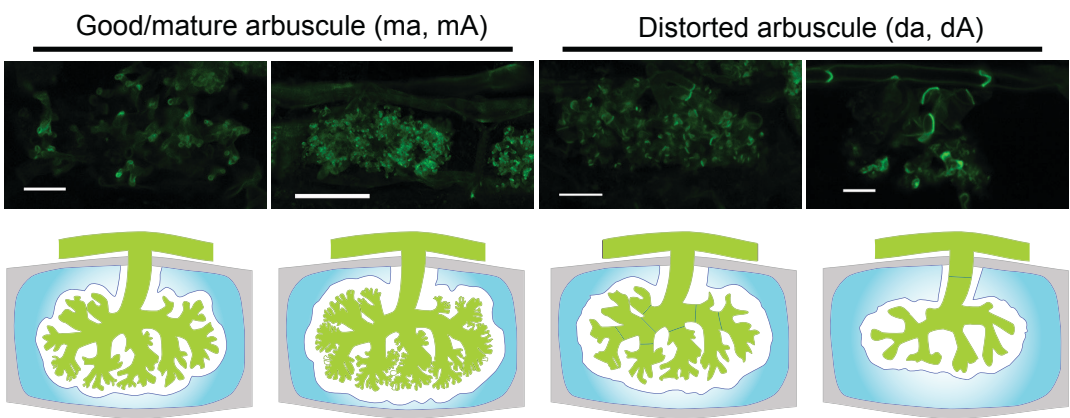

Developing

Mature

Degenerating

Collapsed

(b)

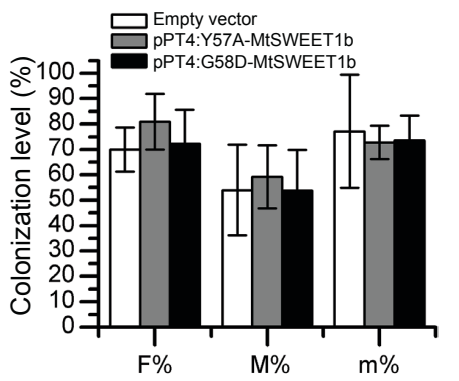

(c)

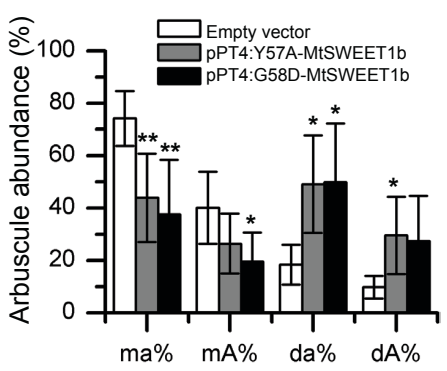

(d)
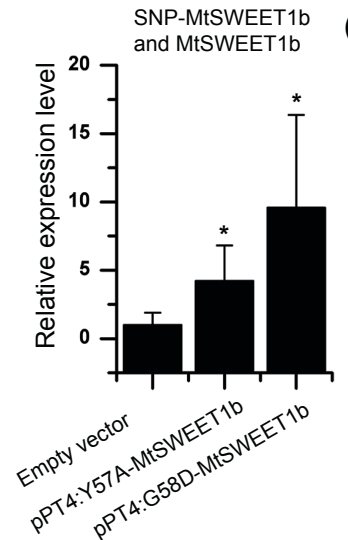

(e)
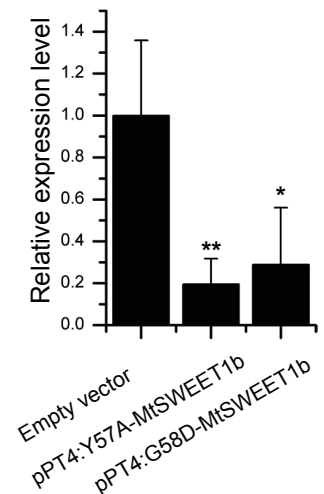

(f)
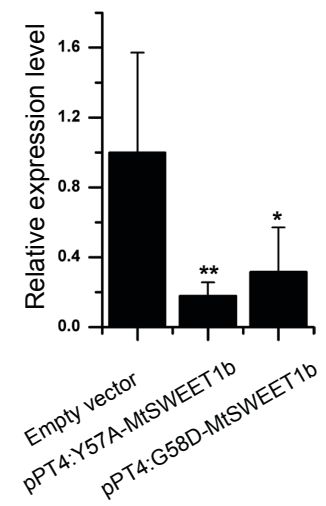

Figure 6. Overexpression of dominant-negative MtSWEET1bY57A/G58D versions in arbuscule-containing cells impairs arbuscule maintenance. (a) Representative confocal images and schematic drawings of WGA-Alexa Fluor 488 stained arbuscule morphology classes: developing arbuscule, bar $=8 \mu \mathrm{m}$; mature arbuscule, bar $=25 \mu \mathrm{m}$; degenerating arbuscule, bar $=8 \mu \mathrm{m}$; collapsed arbuscule, bar $=5 \mu \mathrm{m}$. (b) Mycorrhizal colonization level in dominant-negative lines and empty vector control. Data are averages from biological replicates, $\mathrm{n}$ (empty vector) $=4 ; \mathrm{n}$ (dominant negative Y57A-MtSWEET1b) = 7; $\mathrm{n}$ (dominant negative G58D-MtSWEET1b) $=7$. F\%, the frequency of analyzed root fragments that are mycorrhized; $\mathrm{M} \%$, the intensity of infection in the whole roots; $\mathrm{m} \%$, the intensity of infection in mycorrhized root fragments. White bars = empty vector control roots, grey bars = MtPT4pro:Y57A-MtSWEET1b, black bars = MtPT4pro:G58D-MtSWEET1b. 


\section{A SWEET transporter implicated in AM symbiosis}

To examine the effect of the insertions on the MtSWEET1b transcript level, we performed RT-PCR analysis on RNA from the roots of mycorrhized insertion lines. We found that truncated transcripts of MtSWEET1b could still be detected in both mutants. Sequencing of the truncated cDNA fragments showed in frame deletions of respectively $96 \mathrm{bp}$ and $132 \mathrm{bp}$ in sweet $1 b-1$ and sweet $1 b-2$ (Fig. S8a), likely resulting from alternative splice events skipping the Tnt1 retrotransposon. Similar cases of splicing have been reported in other $M$. truncatula Tnt1-insertion mutants (Krajinski et al., 2014; Kryvoruchko et al., 2016). As a result, a truncated MtSWEET1b protein lacking the second transmembrane region (32 amino acids, 25-57) can still be produced in sweet $1 b-1$, while a truncated protein lacking two transmembrane helices (44 amino acids, 106-149) can be produced in sweet $1 \mathrm{~b}-2$ (Fig. S8b). This is expected to completely disrupt the transport activity of the truncated SWEET proteins.

To rule out that the truncated SWEET1b proteins may still have glucose transport ability, we expressed the truncated mtsweet $1 b-2$ allele in the yeast EBY.VW4000 strain. In contrast to wild-type MtSWEET1b, mtsweet $1 b-2$ was unable to allow the growth of the transformed yeast strain on glucose-containing medium (Fig. S9). The result suggests that the truncated MtSWEET1b protein is indeed no longer able to transport glucose.

To detect whether other glucose transporters would be upregulated at the transcript level to compensate for the loss of MtSWEET1b activity in

continued

(c) Good/mature (m) and degenerating/collapsed arbuscule abundance in mycorrhized roots. ma, Good/mature arbuscule abundance in mycorrhized root fragments; $\mathrm{mA}$, Good/mature arbuscule abundance in the entire root; da, degeneration/dead arbuscule abundance in mycorrhized root fragments; $d A$, degeneration/dead arbuscule abundance in the entire root. Data are averages from biological replicates, $\mathrm{n}$ (empty vector) $=4 ; \mathrm{n}$ (dominant negative Y57A-MtSWEET1b) $=7 ; \mathrm{n}$ (dominant negative G58D-MtSWEET1b) = 7. ${ }^{* *} \mathrm{P}<0.01 ;{ }^{*} \mathrm{P}<0.05$, Student's t-test. (d) qRT-PCR measurement of expression level of mutant versions of MtSWEET1b, detecting both wild-type MtSWEET1b and the transgene mutated $M t S W E E T 1 b$ transcripts in empty vector control lines and dominant-negative lines. (e) Relative expression level of wild-type MtSWEET1b in empty control lines and dominant-negative lines. (f) Relative expression level of MtPT4 in empty vector control lines and dominant-negative lines. (d-f), $n$ (empty vector) $=4$; $n$ (dominant negative Y57A-MtSWEET1b) = 6; $\mathrm{n}$ (dominant negative G58D-MtSWEET1b) $=6$. * $P<0.01 ;{ }^{*} P<0.05$, Student's t-test. MtEF1 $\alpha$ was used as reference gene. 


\section{CHAPTER 5}

sweet $1 b$ mutants, we detected the expression of all other SWEET genes in sweet $1 b-1$ mutants and wild type R108 by qRT-PCR. Our results showed that none of the other SWEET gene was significantly up-regulated in the sweet1b-1 mutant compared to the wild-type control (Fig. S10). Although a potential slight compensation at the expression level might have gone undetected in the whole (mycorrhized) root samples, a lack of transcriptional compensation was also reported for MtSWEET11, which is highly expressed in root nodules but for which Tnt 1 mutants similarly failed to reveal a symbiotic phenotype (Kryvoruchko et al., 2016). Furthermore, it should be noted that we cannot rule out a potential compensation at the protein level.

\section{Expression of dominant-negative MtSWEET1b alleles impairs the maintenance of arbuscules}

Previous studies revealed that SWEET transporters function in multimers (Xuan et al., 2013; Tao et al., 2015). Two amino acids (Y57 and G58) were reported to be essential for the AtSWEET1 glucose transport activity in Arabidopsis and these mutant forms were shown to act in a dominantnegative manner when overexpressed (Xuan et al., 2013). Both the amino acids are conserved between MtSWEET1b and AtSWEET1 (Fig. S11). To determine whether the two residues are important for glucose transport in MtSWEET1b, we constructed MtSWEET1b mutants carrying either the Y57A or G58D substitution. Introduction of these mutant versions into EBY.VW4000 did not allow yeast growth on media containing glucose as the sole carbon source (Fig. S12), indicating that these two protein variants indeed lost their ability to transport glucose.

We then expressed GFP-tagged versions of MtSWEET1 $b^{\text {Y57A }}$ and MtSWEET1 $b^{G 58 D}$ from the strong arbuscule-specific MtPT4 promoter to study their subcellular localization. After transformation into $M$. truncatula roots, the composite plants were inoculated with the $R$. irregularis and analyzed by confocal microscopy. Both the MtSWEET1 $b^{\text {Y57A }}$ and MtSWEET1 ${ }^{G 58 D}$ GFP fusion proteins could be detected on the periarbuscular membrane, similar to that of wild type MtSWEET1b-GFP (Fig. S13).

Next, we specifically overexpressed non-tagged MtSWEET $1 b^{\text {Y57A }}$ and MtSWEET1b ${ }^{G 58 D}$ in arbuscule-containing cells using the MtPT4 promoter 
and quantified mycorrhization levels compared to EV control roots at 6 wpi. Higher expression levels of MtSWEET1 $b^{\text {Y57A }}$ and MtSWEET1 $b^{G 58 D}$ were detected in the relevant transgenic lines compared to that of the wild-type gene in the EV control line (Fig. 6d). Both the frequency and intensity of mycorrhization and number of arbuscules were not significantly affected in these transgenic lines in comparison with the EV control (Fig. 6b). To assess whether the morphology of arbuscules was affected due to overexpression of the two dominant-negative alleles, we again classified arbuscules into good/mature arbuscules (Fig. 6a left two panels) and degenerating/collapsed arbuscules (Fig. 6a right two panels). Interestingly, we observed a clear difference in arbuscule morphology between the transgenic lines and the EV control: there were significantly more degenerating/collapsed arbuscules (da\%) and fewer healthy mature arbuscules ( $\mathrm{ma} \%$ ) in the transgenic lines expressing either of the dominant negative alleles when compared with the EV control (Fig. 6c). Similar results were obtained in a second independent experiment (Fig. S14). In line with the microscopic quantification, transgenic roots with a lower frequency of healthy mature arbuscules also showed lower expression levels of MtPT4 (Fig. 6f) and the wild type MtSWEET1b (Fig. 6e). These results suggest that MtSWEET1b can form oligomers with itself and other SWEET proteins to provide glucose required for arbuscules maintenance. However, we cannot rule out that the truncated MtSWEET1b proteins in the Tnt1-lines retain an activity in transporting substances other than glucose for arbuscule maintenance.

\section{Discussion}

In this study we found that MtSWEET1b encodes a glucose transporter that is strongly upregulated in arbuscule-containing cells compared to roots and which localizes to the peri-arbuscular membrane surrounding the arbuscules (Fig. 7). Overexpression of MtSWEET1b increased the fungal mass in symbiotic roots, further suggesting that MtSWEET1b may play a role in exporting sugar from arbuscule-containing cells to AM fungi (Fig. 7). However, mycorrhization did not seem to be significantly affected in two M. truncatula lines where the MtSWEET1b gene is mutated to produce a truncated protein that is incapable of glucose transport in yeast. Intriguingly, overexpression of dominant-negative alleles of MtSWEET1b impaired arbuscule maintenance, implying that MtSWEET1b might form hetero-oligomers with other (SWEET) transporters. 


\section{CHAPTER 5}

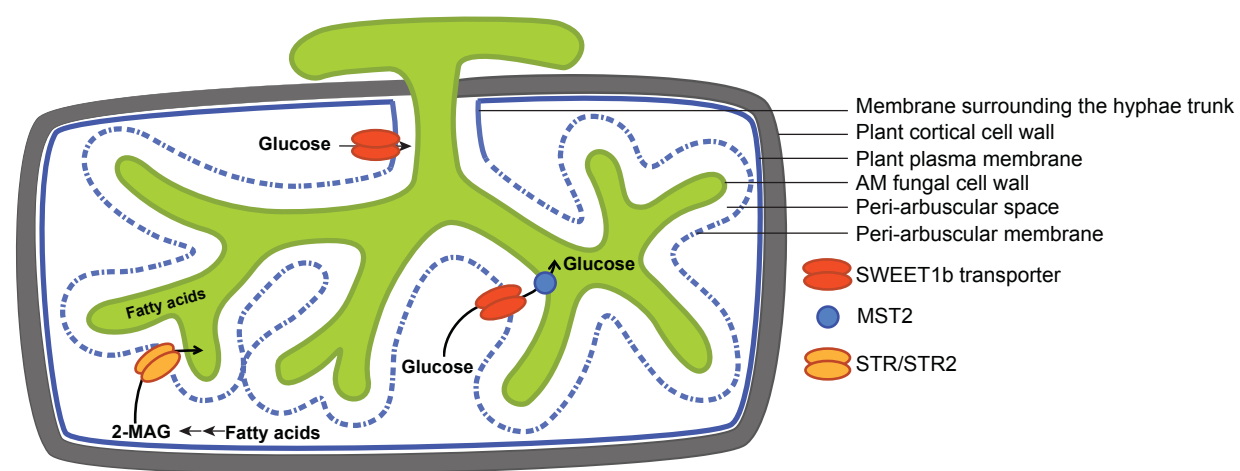

Figure 7. Proposed model for M. truncatula SWEET1b function in the arbusculecontaining root cortical cell during AM symbiosis.

M. truncatula has 26 SWEET genes, of which only MtSWEET1b showed strongly induced expression in root cortex cells colonized by the AM fungus. Several other MtSWEET members did show differential expression upon mycorrhization, however none of them exhibited induced expression in the root cortex cells where nutrient/sugar delivery is thought to occur (Roth and Paszkowski, 2017). Phylogenetic analyses showed that MtSWEET1b is a homolog of AtSWEET1, a glucose transporter mainly active in flowers (Chen et al., 2010). M. truncatula has two homologs in this clade, of which MtSWEET1a is highly expressed in the flowers. There are at least two closely related SWEET1 homologs in O. sativa, $L$. japonicus or S. tuberosum. Interestingly, at least one SWEET1 in each plant species was found to be induced upon mycorrhization (Handa et al., 2015; Manck-Götzenberger and Requena, 2016; Sugimura and Saito, 2017). It seems likely that Arabidopsis, a non-host for AM fungi, has lost the AM regulated gene. In potato, several additional SWEET genes were found to be induced upon mycorrhization in arbuscule-containing cells, including clade II and clade III (sucrose transporter) SWEET genes (Manck-Götzenberger and Requena, 2016). Furthermore, in potato a clade I homolog of AtSWEET2 was also found to be induced in arbusculecontaining cells, which is thought to function at the tonoplast (Chen et al., 2015). This indicates that regulation of SWEET gene expression in different plant species may differ, and we speculate that it will influence the symbiotic efficiency of different plant-fungal combinations. Besides increased expression in arbuscule-containing cells, MtSWEET1b is also highly expressed in root nodules, seeds and pods (Kryvoruchko et al., 2016), raising the suggestion that it may play an important role in both 
AM and rhizobium symbioses.

The phylogenetic analyses suggested that MtSWEET1b is a glucose transporter. This was confirmed in yeast, as MtSWEET1b could complement the glucose transport defect of the EBY.VW4000 mutant (Wieczorke et al., 1999). So far, all the functionally characterized SWEET family members from different plant species were confirmed to have both sugar efflux and influx activity (Chen et al., 2012; Chen et al., 2010; Lin et al., 2014b; Eom et al., 2015). As uniporters SWEETs transport sugars along a concentration gradient, which raises the question whether MtSWEET1b exports glucose towards the fungus, or whether it imports glucose in competition with the fungus to support the high metabolic activity of arbuscule-containing cells or to avoid defense responses caused by high levels of glucose in the apoplast (Schaarschmidt et al., 2007; Helber et al., 2011; Moore et al., 2015; Bezrutczyk et al., 2018). Several studies indicate that arbuscules-containing cells form a strong sink for hexoses. For example, a hexose transporter (MtHEX1) likely involved in the uptake of hexose from the apoplast and a sucrose transporter (MtSUT1) thought to be involved in the export of sucrose from the vacuole as well as genes encoding amylase and secreted invertases showed the highest level of expression in root cortex cells adjacent to arbuscule-containing cells (Gaude, Bortfeld, et al., 2012; Gaude, Schulze, et al., 2012). The observation that plasmodesmata between arbuscule-containing cells and adjacent cells are enlarged (Blee and Anderson, 1998), suggests that cortical cells adjacent to arbuscule-containing cells supply carbohydrates to arbuscule-containing cells via symplastic transport (Gaude, Schulze, et al., 2012). In the arbuscule-containing cells, a strongly induced cytoplasmic sucrose synthase (MtSucS1) converts the sucrose to glucose. When knocked-down, this gene impaired fungal colonization and caused an early collapse of arbuscules (Baier et al., 2010). Therefore, we consider it likely that the glucose concentration inside arbuscule-containing cortex cells is higher than in the peri-arbuscular space and that MtSWEET1b facilitates the export of glucose across the peri-arbuscular membrane for subsequent uptake by the fungus through the monosaccharide transporter MST2 (Fig. 7) (Helber et al., 2011). Recently, it has been shown that the expression of MtSWEET1b is consistent with a carbon allocation to the fungal side during AM symbiosis (Kafle et al., 2019), which strengthens the possibility that MtSWEET1b indeed exports sugars 


\section{CHAPTER 5}

to AM fungi. However, formal proof that MtSWEET1b indeed exports glucose across the peri-arbuscular membrane remains to be shown, which is complicated by the fact that the fungus also obtains carbon in the form of fatty acids that can be converted into sugars by the fungus (Jiang et al., 2017; Luginbuehl et al., 2017).

The observation that overexpression of MtSWEET1b increased the mycorrhization level may suggest that more glucose was supplied to the AM fungi thereby promoting the growth of the fungus inside the roots. However, the absence of any effect on arbuscule formation or mycorrhization levels in the Tnt1 MtSWEET1b insertion lines challenged the importance of glucose efflux into AM fungi during AM symbiosis. The insertion lines could still produce truncated MtSWEET1b proteins, which may still retain certain transport activity that could explain the lack of mycorrhization phenotypes. However, we consider it very unlikely, since the predicted deletion of transmembrane domains would seriously disrupt the protein configuration in the membrane. Expression of such a truncated MTSWEET1b protein did not allow the hexose-transport deficient EBY. VW4000 yeast strain to grow on glucose-containing medium, indicating that at least the glucose transport ability of MtSWEET1b is indeed lost. Thus, our genetic data indicate that the AM fungus is still able to obtain sufficient carbohydrates to sustain fungal growth in host plants in which glucose transport activity derived from MtSWEET1b is lost. This further implies that there is functional redundancy between MtSWEET1b and other related SWEET family members or that there is compensation through sugar uptake from the apoplast by the intraradical hyphae (Helber et al., 2011; Roth and Paszkowski, 2017)

Intriguingly, we found that overexpression of a presumed dominantnegative forms of MtSWEET1b in wild-type M. truncatula plants caused an early collapse of arbuscules even though there was no significant reduction in intraradical hyphal growth or total amount of arbuscules. This suggests that the MtSWEET1b mutant forms poise complexes with other (SWEET) transporters, affecting the transport of sugars or other substances required for arbuscule maintenance. It was recently found that clade III SWEET transporters in Arabidopsis can transport gibberellic acid (GA) (Kanno et al., 2016). GA enhances the degradation of DELLA proteins and is an important negative regulator of symbiotic signaling (Pimprikar et al., 2016). Whether MtSWEET1b can transport GA, or other 
metabolites, remains to be investigated.

Taken together, our results provide a first indication that SWEET transporters play an important role to stabilize the AM symbiosis (Kiers et al., 2011). Understanding how costs and benefits are monitored and modulated by both partners will be key to improve plant performance through AM symbiosis.

\section{Acknowledgements}

We wish to thank Dr Liqing Chen (Illinois, USA) and Dr Francois Houllier (INRA, France) for providing plasmids ofpDR-F1-GW and PPMA1, respectively. We thank Dr Eckhard Boles (Goethe-Universität Frankfurt am Main) for providing yeast mutant EBY.VW4000. This work was supported by the National Key Research and Development Program of China (nos. 2018YFD1000103; 2017YFD0202001), the National Natural Science Foundation of China (no. 31521092), the Science and Technology Major Project of Guangxi (Gui Ke AA 18118046), the High-End Foreign Experts Project (no. GDT20174200001), the Fundamental Research Funds for the Central Universities (no. 2662018JC039), the European Research Council (ERC-2011-AdG294790) and Koninklijke Nederlandse Akademie van Wetenschappen (KNAW, no. 530-6CDP21).

\section{Supplementary data}

Supplementary tables listed below can be downloaded from the New Phytologist website: https://doi.org/10.1111/nph.15975

Table S1. Primer sequences used in this study.

Table S2. List of M. truncatula SWEET family genes (accession numbers). 
CHAPTER 5

\section{Supplementary figures}

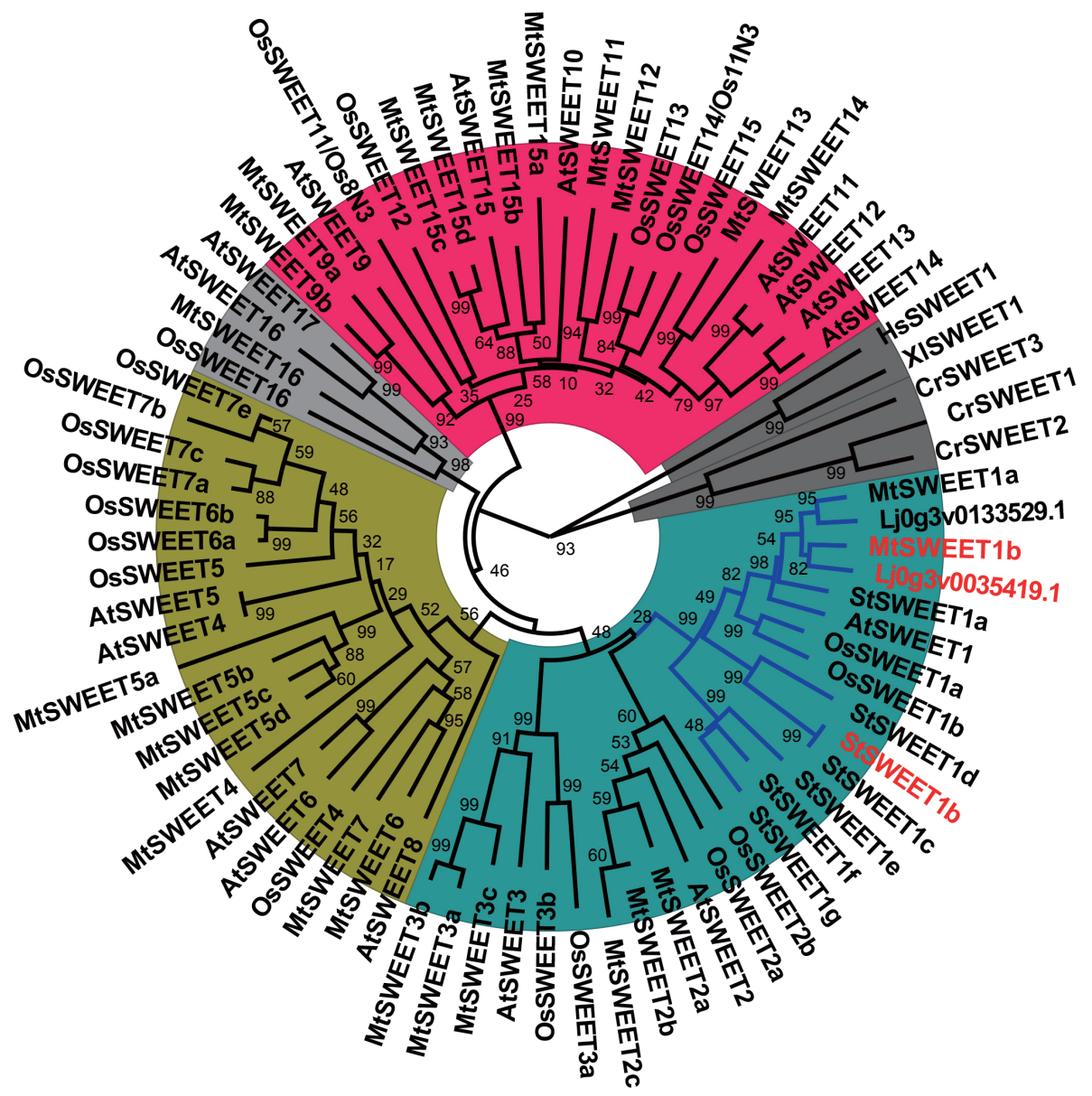

Figure S1. Phylogenetic tree of SWEET family genes. Species included are $A$. thaliana (At), M. truncatula (Mt), O. sativa (Os), L. japonicus ( $\mathrm{Lj}$ ) and $S$. tuberosum (St). The protein sequences were aligned by ClustalW and a neighbor-joining phylogenetic tree was generated based on the alignment. Branch support was obtained from 2000 bootstrap repetitions. Figtree v1.4.2 was used to adjust the shape of the phylogenetic tree. The five SWEET clades are marked by different colours: Clade I, blue-green; Clade II, golden yellow; Clade III, rose red; Clade IV, light grey; Clade V, dark grey. Clade I SWEET genes (marked blue) highlighted in red are induced in AM symbiosis. 


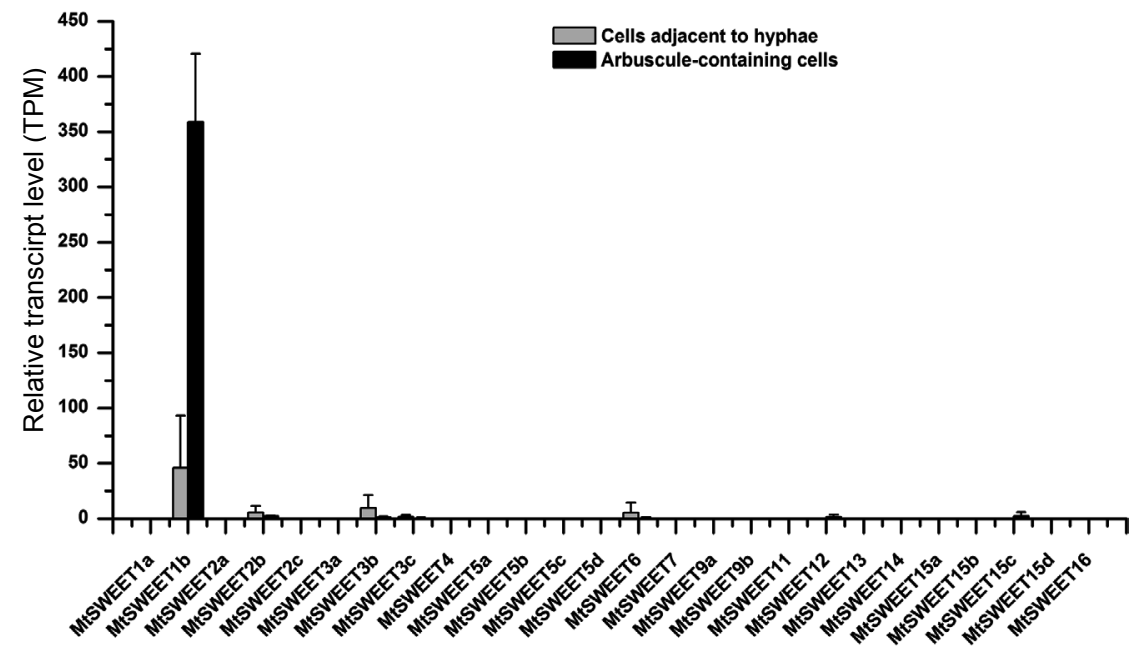

Figure S2. Analysis of the expression pattern of $M$. truncatula SWEET family genes during AM symbiosis. Expression levels (TPM) of $M$. truncatula SWEET family genes in cortical cells containing arbuscules and cortical cells adjacent to intercellular hyphae are based on RNA-seq data by Zeng et al. (2018). TPM, transcripts per million. Data are represented as the mean $\pm S D$ of three biological replicates. 
(a)

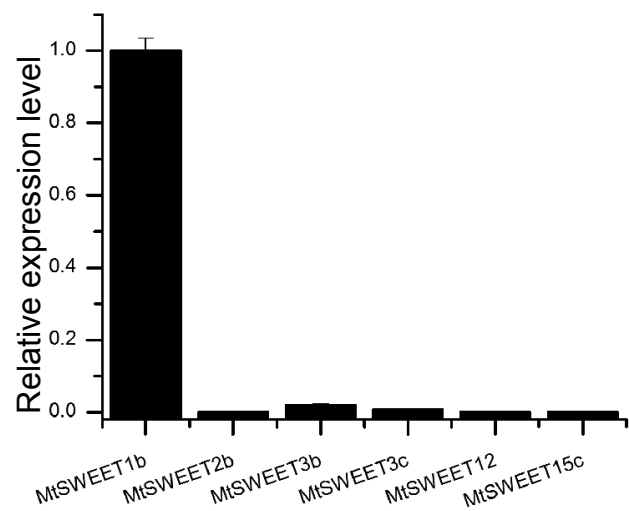

(b)

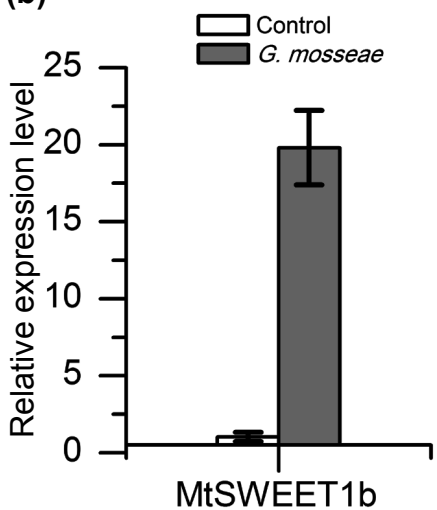

Figure S3. Relative expression level of MtSWEET genes in the arbusculecontaining cells and roots inoculated with $F$. mosseae. (a) Relative expression level of MtSWEET genes in arbuscule-containing cells isolated from mycorrhizal roots by laser capture microdissection. Data are averages from three biological replicates. Error bar shows SD. The values were calculated using the 2- $\Delta \Delta \mathrm{Ct}$ method relative to MtSWEET1b. MtEF1a was used as reference gene. (b) Expression level of MtSWEET1b in M. truncatula non-inoculated control roots or roots inoculated with F. mosseae at 6 wpi. The values were calculated relative to non-inoculated control samples. MtEF1 $\alpha$ was used as reference gene. Error bars \pm SD were obtained from three independent biological replicates.

(a)

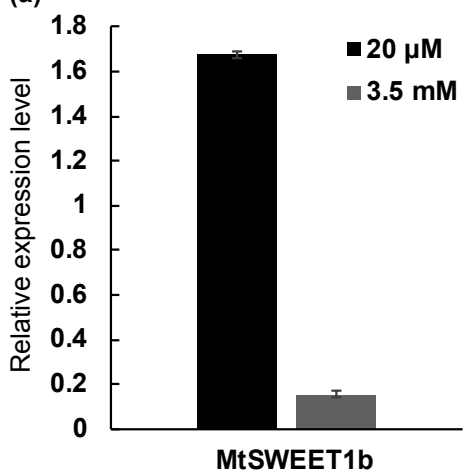

(b)

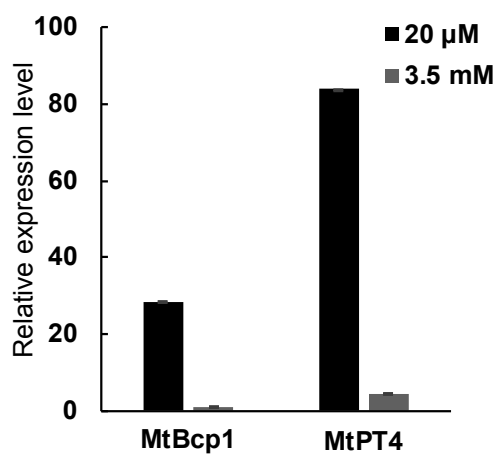

Figure S4. Expression levels of MtSWEET1b, MtBCP1 and MtPT4 in M. truncatula roots in response to high concentration of phosphate. (a-b) Relative expression level of MtSWEET1b (a) and (b) MtPT4 and MtBCP1 in M. truncatula roots inoculated with $R$. irregularis, grown for 4.5 weeks under low (20 $\mu \mathrm{M})$ phosphate condition after which the phosphate concentration was raised to 3.5 $\mathrm{mM}$ for 3 days. SD indicates standard deviation from the mean of three biological replicates. The values were calculated using the 2- $\Delta \Delta \mathrm{Ct}$ method relative to the expression of MtSWEET1b under low (20 $\mu \mathrm{M})$ phosphate condition, MtEF1a was used as reference gene. 


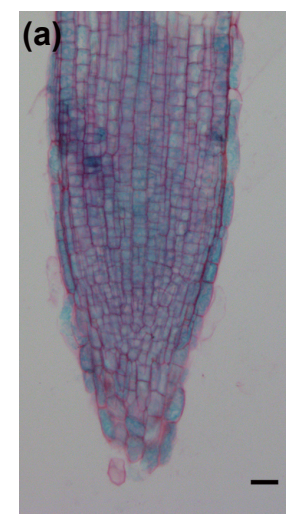

R. irregularis

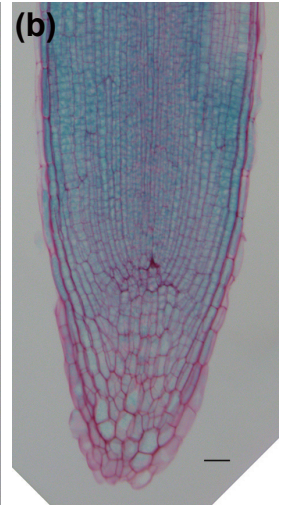

Non-inoculated

Figure S5. MtSWEET1b promoter activity in M. truncatula root tips. (a-b) Hairy roots transformed with MtSWEET1bpro:GUS inoculated (a) and non-inoculated (b) with $R$. irregularis, showing promoter activity in the root tip. Bars: (a) $25 \mu \mathrm{m}$, (b) 75 $\mu \mathrm{m}$.

(a)

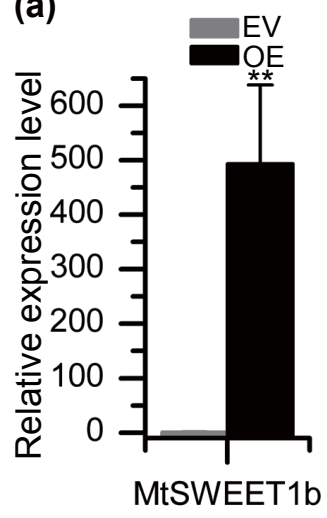

(b)

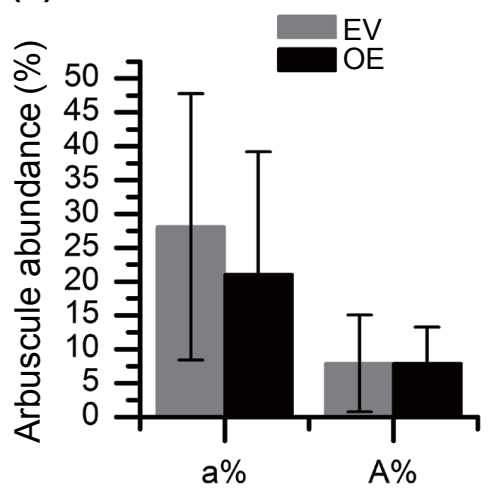

(c)

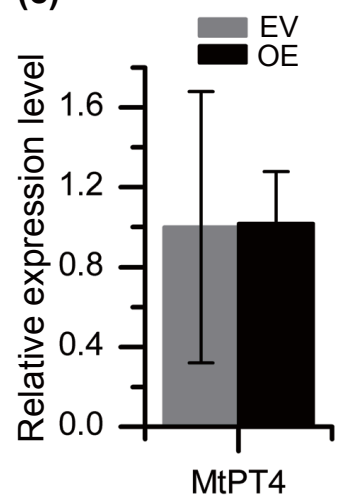

Figure S6. Relative expression levels of MtSWEET1b and MtPT4 and arbuscule abundance in MtSWEET1b overexpression and empty vector transgenic M. truncatula roots. (a and c) Expression levels of MtSWEET1b (a) and MtPT4 (c) in $M$. truncatula roots overexpressing MtSWEET1b or empty vector control roots determined by qRT-PCR analyses at 5 wpi with R. irregularis. EV, empty vector lines. OE, MtPT4p:MtSWEET1b lines. MtEF1a was used as reference gene. Relative expression levels are normalized against the EV lines using the 2- $\Delta \Delta \mathrm{Ct}$ method. Data are represented as the mean $\pm S D$ of six biological replicates. ${ }^{* *} \mathrm{P}<$ 0.01 , Student's t-test. (b) Quantification of arbuscule abundance in M. truncatula roots overexpressing MtPT4p:MtSWEET1b and empty vector control roots at 5 wpi with $R$. irregularis. The parameters $a \%$ and $A \%$ indicate arbuscule abundance in mycorrhized root fragments and arbuscule abundance in the entire root system, respectively. Data are represented as the mean $\pm S D$ of six biological replicates. ${ }^{* *}$ $P<0.01$, Student's t-test. 
(a)

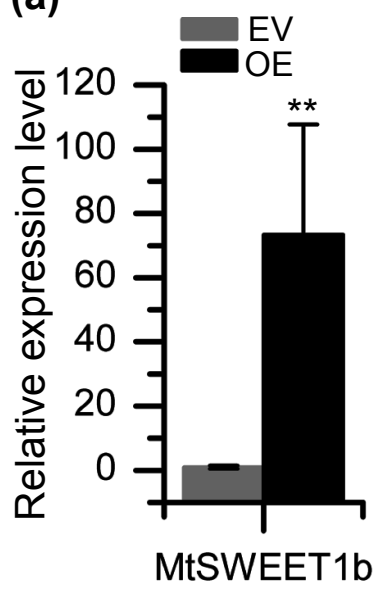

(b)

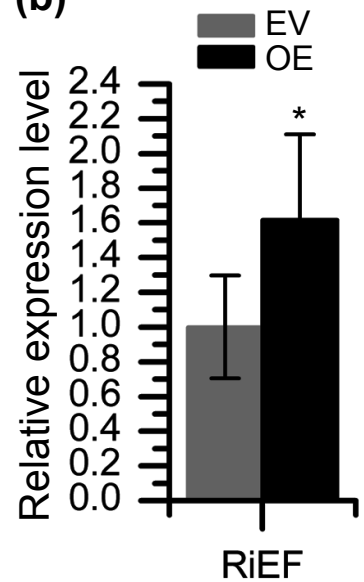

(c)

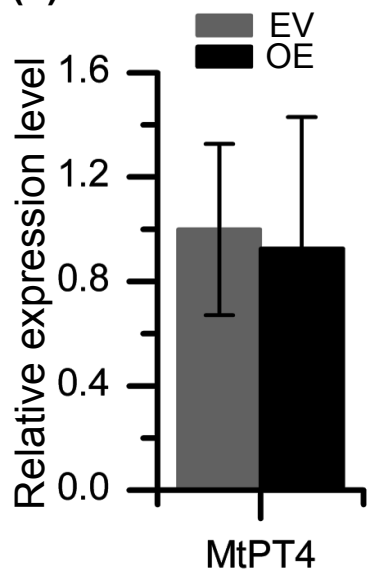

Figure S7. Relative expression of MtSWEET1b, RiEF and MtPT4 in a second independent MtSWEET1b overexpression experiment. (a-c) The expression levels of MtSWEETb (a), RiEF (b) and MtPT4 (c) in M. truncatula roots expressing MtPT4pro:MtSWEET1b and empty vector control roots were measured by qRT-PCR at 5 wpi with R. irregularis. EV, empty vector lines. OE, MtPT4pro:MtSWEET1b lines. $M t E F 1 \alpha$ was used as reference gene. Relative expression levels were normalized against the EV lines using the $2-\Delta \Delta C t$ method. Data are represented as the mean $\pm S D$ of five biological replicates . ${ }^{* *} \mathrm{P}<0.01,{ }^{*} \mathrm{P}<0.05$, Student's t-test. 


\section{A SWEET transporter implicated in AM symbiosis}

(a)

$>$ MtSWEET1b coding sequences

atgcatgttgcacatctttgtttggcatatttgggaatgcttctgcttgttcctcttcttggcacctgtgattacattcaa gaggattatagtcaacaaatccacagagaaattttcaggcttaccatatgttatgactctgctcaattgtctcctttc tgcttggtacggtctgccttttgtgtctcccaacaacataccggtaacaacagtaaatggcaccggagcaggg attgaaatcatatatgtttgatattcatcatatttgcacctaagaaggaaaaaatcaaaatctttgccctattcacc ttggtactgtctgtgttctctgctgttgttttgtctccctctttgccttccatggcaatcataggaaggccttctgcggctt tgccatggctatattttccgtcatcatgtatggttctccactctcaattatgagattagtgatcaaaaccaagagtgt ggagttcatgccattcttcttgtcgctgtttgtgtttcttgcggcagttcatggtttatattcggtctgctaggccgtgac ctatttgttgctgtacctaatggtcttggttctgtttggggacaatgcaactaatattgtattcatataccgtgacaa caaaggtagtccaaaacagcaggaaccaacagaaggggaatcaatggagatgggtaatggaaaaaacc atcaaatgaaacagtcttatgaaaatgaaattcaaggatga

$96 \mathrm{bp}$ deletion of MtSWEET1b transcripts in sweet1b-1 mutant

132 bp deletion of MtSWEET1b transcripts in sweet $1 \mathrm{~b}-2$ mutant

(b)

MtSWEET1b
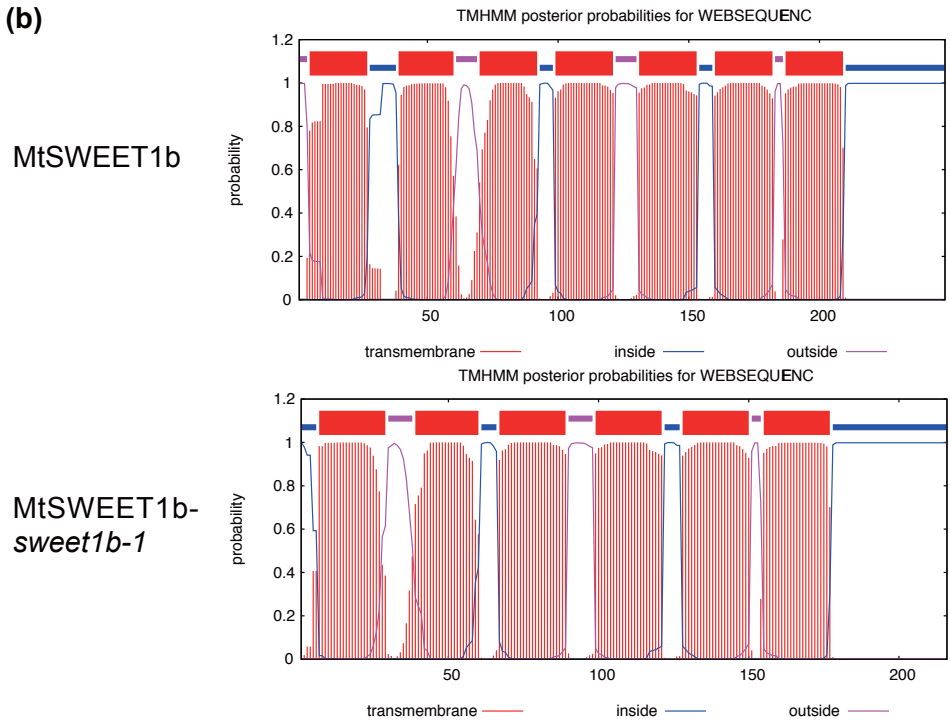

TMHMM posterior probabilities for WEBSEQUENC

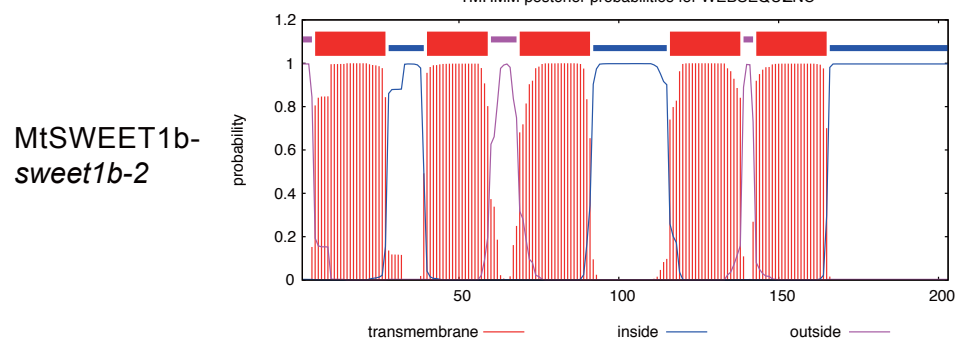

Figure S2. MtSWEET1b truncated transcripts detected in sweet1b-1 and sweet1b-2, and protein transmembrane domain prediction. (a) Representation of the deletion regions in the MtSWEET1b transcripts detected in sweet $1 \mathrm{~b}-1$ and sweet1b-2. The $96 \mathrm{bp}$ red colour region is deleted in the sweet1b-1 (NF1309) mutant. The 132 bp blue coloured sequences are deleted in the sweet1b-2 (NF3539) mutant. (b) Protein transmembrane structure prediction of wild-type MtSWEET1b and truncated MtSWEET1b proteins predicted for sweet $1 b-1$ and sweet $1 b-2$. 


\section{CHAPTER 5}
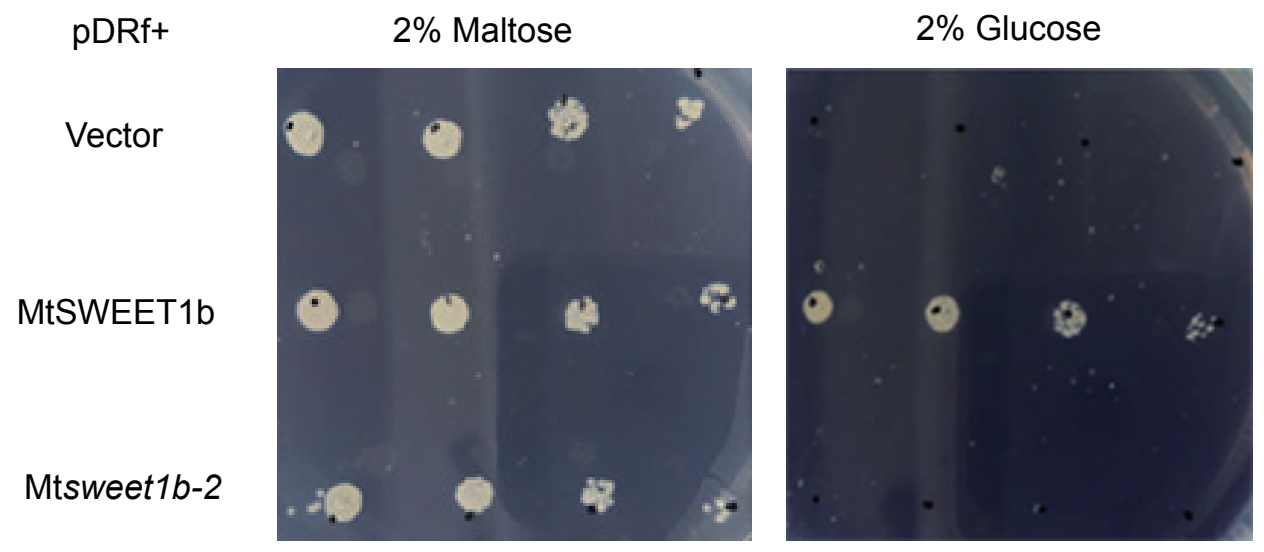

Figure S9. Functional analysis of sweet $1 \mathrm{~b}-2$ by complementation of the yeast hexose deficiency mutant strain EBY.VW4000. The yeast hexose transport defective strain EBY.VW4000 was transformed with pDRf-1 empty vector, pDRf-1MtSWEET1b, and pDRf-1-Mtsweet1b-2 (132bp deletion), respectively. The images show the growth ability of the transformed EBY.VW4000 strains on SD media containing $2 \%$ maltose or $2 \%$ glucose at $30^{\circ} \mathrm{C}$ after 3 days. Empty vector and wildtype MtSWEET1b transformed yeast were used as negative and positive control, respectively. In each image, the yeast strain was diluted to an OD600 of 0.2 and subsequently $1 \times, 5 \times, 25 \times$ and $125 \times$ dilutions were spotted. 


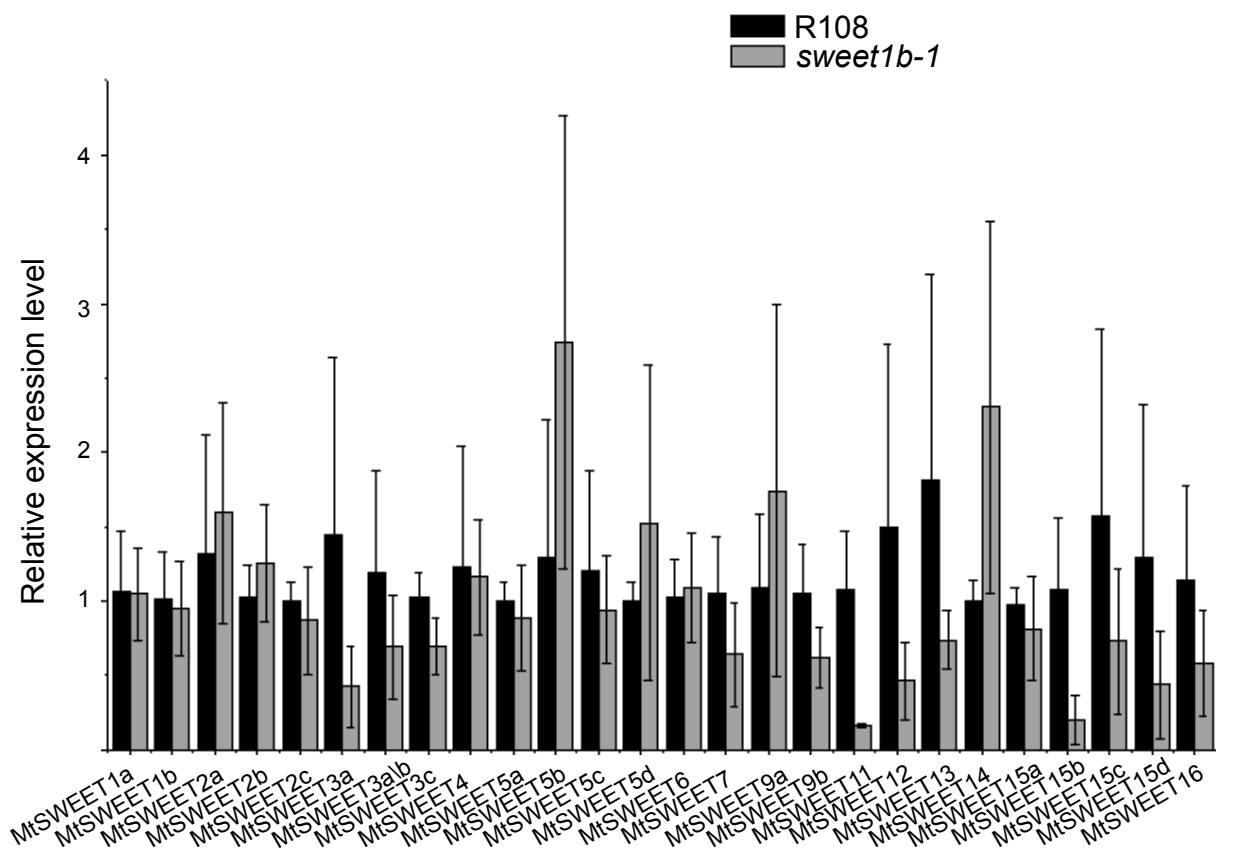

Figure S10. Expression levels of $M$. truncatula SWEET family genes in mycorrhized roots of wild type R108 and sweet1b-1. Roots were harvested at 5 wpi with $R$. irregularis. MtEF1 $\alpha$ was used as reference gene. Expression values were normalized against the level in wild-type R108 AM roots with the 2- $\Delta \Delta \mathrm{Ct}$ method. The annealing sites of the qRT-PCR primers for MtSWEET1b used in this experiment are perserved in the truncated transcript in sweet $1 \mathrm{~b}-1$. Data are represented as the mean $\pm S D$ of biological replicates and each biological sample (n) contained 3 technical replicates. n (SWEET1b, SWEET3a/b, SWEET12, SWEET15c, SWEET15d)R108 $=7, \mathrm{n}($ SWEET1b, SWEET3a/b, SWEET12, SWEET15c, SWEET15d)sweet1b-1 = $9, \mathrm{n}$ (others) $=2$. 


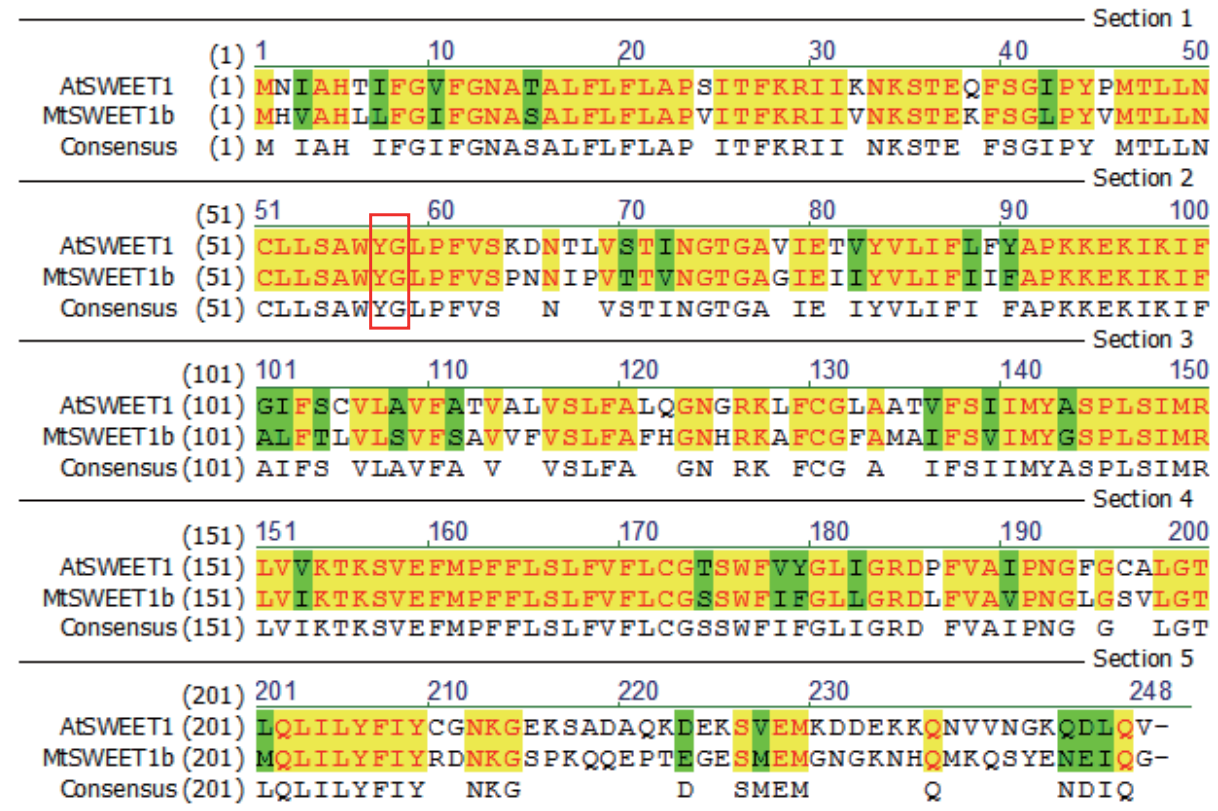

Figure S11. Protein sequence alignment of MtSWEET1b and AtSWEET1. Two conserved amino acid residues (57Y and $58 \mathrm{G}$ ) required for sugar transport activity are highlighted by a red box.

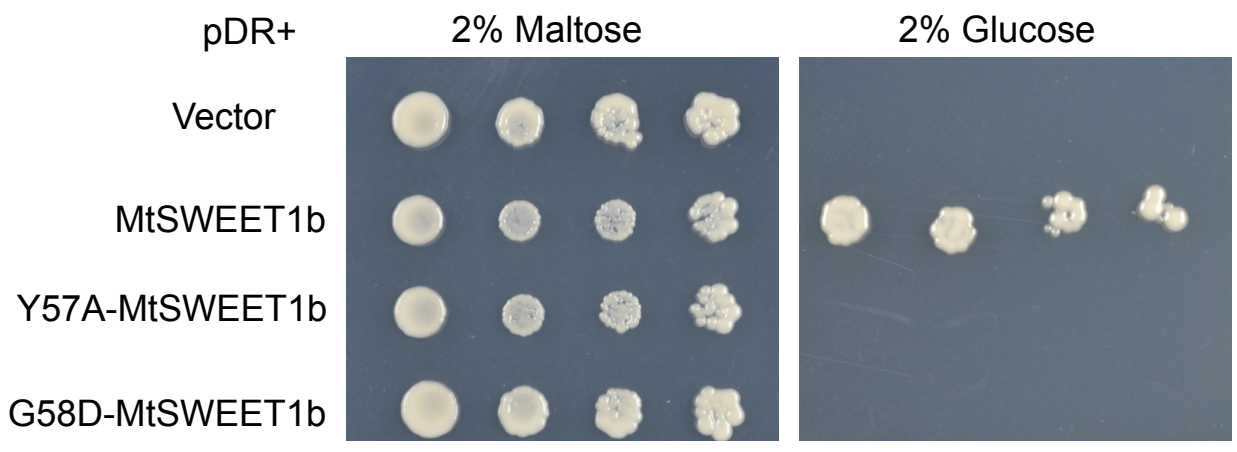

Figure S12. Functional analysis of MtSWEET1bY57A/G58D mutants by complementation of yeast hexose deficiency mutant strain EBY.VW4000. The yeast hexose transport defective strain EBY.VW4000 was transformed with pDRf-1 empty vector, pDRf-1-MtSWEET1b, pDRf-1-Y57A-MtSWEET1b and pDRf1-G58D-MtSWEET1b, respectively. The images show the growth ability on SD media containing $2 \%$ maltose or $2 \%$ glucose at $30^{\circ} \mathrm{C}$ for 3 days. Empty vector and MtSWEET1b transformed yeast were used as negative and positive control, respectively. In each image, the yeast strain was diluted to an OD600 of 0.2 and subsequently spotted at $1 \times, 5 \times, 25 \times, 125 \times$ dilutions with sterile water. 

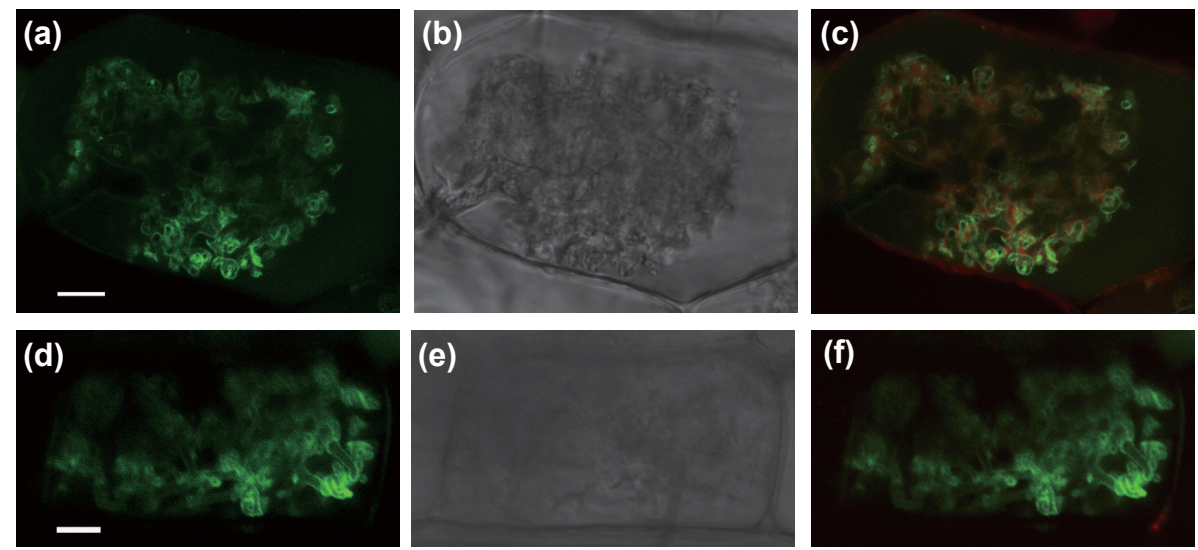

Figure S13. Subcellular localization of Y57A-MtSWEET1b-GFP and G58DMtSWEET1b-GFP in arbuscule-containing cells. (a-f) Confocal microscopy images of $M$. truncatula roots expressing MtPT4pro:Y57A-MtSWEET1b-GFP (ac) or MtPT4pro:G58D-MtSWEET1b-GFP (d-f) inoculated with $R$. irregularis. GFP fluorescence of Y57A-MtSWEET1b-GFP (a) and G58D-MtSWEET1b-GFP (d) is present on the peri-arbuscular membrane. ( $b$ and $e$ ) Corresponding brightfield images. (c and f) Merged green and red channels. Red fluorescence in the cytoplasm was from the DsRed protein expressed under the control of the A. thaliana Ubiquitin3 promoter. Bars $=8 \mu \mathrm{m}$.

(a)

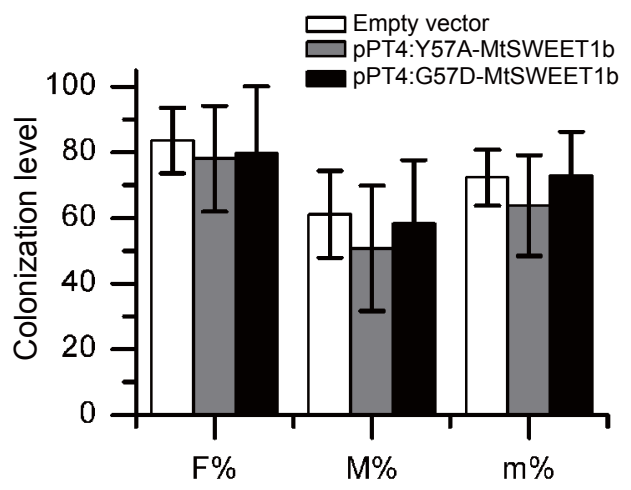

(b)

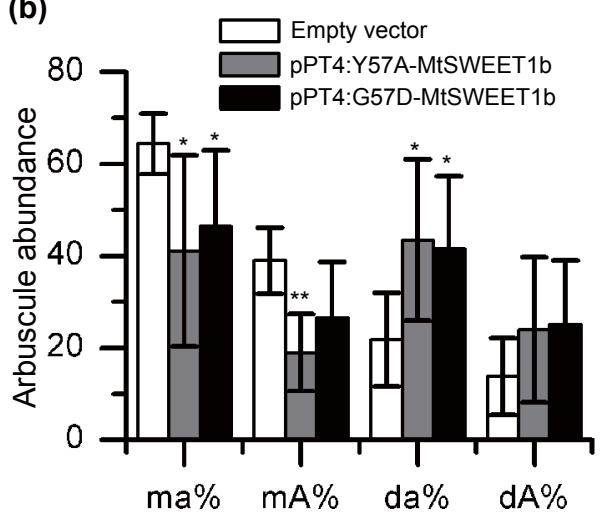

Figure S14. Independent repetition of the overexpression of MtSWEET1bY57A/ G58D dominant-negative constructs. (a-b) Mycorrhizal colonization levels (a) and arbuscule abundances (b) in dominant-negative and empty vector control lines. Roots were harvested at 6 wpi with $R$. irregularis. Data are represented as the mean $\pm S D$ of biological replicates; $n$ (empty vector) $=5$; $n(p P T 4: Y 57 A-M t S W E E T 1 b)=$ 10; n (pPT4:G58D-MtSWEET1b) = 8. Good/mature $(\mathrm{m})$ and degeneration/dead $(\mathrm{d})$ arbuscule abundance. ma\%, good/mature arbuscule abundance in mycorrhized roots; $\mathrm{mA} \%$, good/mature arbuscule abundance in whole roots; da $\%$, degeneration/ dead arbuscule abundance in mycorrhized roots; dA\%, degeneration/dead arbuscule abundance in whole roots. ${ }^{*} \mathrm{P}<0.05$; ${ }^{* *} \mathrm{P}<0.01$, Student's t-test. 


\section{Chapter 6}

\section{General discussion}

How do AM fungi achieve their extremely broad host range? 


\section{CHAPTER 6}

AM fungi (AMF) have the striking ability to establish an endosymbiosis with the vast majority of all land plants. How AM fungi evolved to colonize such a wide range of hosts is a fundamental question in AM biology. Two aspects are critical in this regard. First, the fungus has to deal with the host immune system to prevent a strong defense response. Second, the fungus has to offer crucial benefits to its hosts otherwise the interaction would be selected against and the symbiosis would not be maintained. Most molecular insights into these aspects have been obtained from the plant side. However, with the recent availability of the first AM fungal genome sequence, substantial progress has been made to get insight into the fungal aspects of this interaction. In this thesis I have used (cell-specific) transcriptome analyses to obtain insight into both fungal and plant components that are regulated in a host- and stage- (or cellspecific) manner. In this chapter I will discuss our recent insights in the light of two main questions, namely, 1) how do AM fungi deal with the plant immune system and 2 ) how is (reciprocal) nutrient exchange controlled.

\section{How do AM fungi evade the plants immune system?}

Like other fungi, AMF have common fungal microbe-associated molecular patterns (MAMPs) such as chitin and $\beta$-glucan in their cell walls, which are known triggers of innate immune responses in plants (Rovenich et al., 2016; Fesel and Zuccaro, 2016). Indeed, AMF (or culture filtrates) have been shown to initially trigger defense responses in the plant, which are subsequently rapidly quenched (García-Garrido and Ocampo, 2002). This indicates the release of fungal components that subvert the plant immune system. In addition to an active influence on the immune system, AM fungi generally lack genes encoding cell wall degrading enzymes to avoid the generation of damage-associated molecules that trigger defense (Tisserant et al., 2013). Therefore, AM fungi rely on their hosts to degrade the cell wall in a controlled manner to facilitate root colonization. Loss of cell wall degrading enzymes seems to be a general strategy used by mutualistic fungi that colonize plants (Kohler et al., 2015). Below, I summarize several ideas of how AM fungi may subvert the host immune system. 
To initiate the symbiosis, AM fungi release chitin-like signal molecules called myc-factors. These include both lipo-chitooligosaccharides (LCOs) and short-chain chito-oligosaccharides (COs, such as CO4) (Maillet et al., 2011; Genre et al., 2013) and both are thought to be perceived by complexes of LysM-domain containing receptor-like kinases (LysM-RLKs). One of the key outputs of symbiotic LysM-RLK activation, in complex with another LRR-RLK called SYMRK/DMI2 (Ried et al., 2014), is the induction of calcium oscillations in the nucleus. These are decoded by a calcium- and calmodulin dependent kinase (CCaMK), which is required to activate a symbiosis-specific transcriptional program (for details see Chapter 1). LysM-RLK's are part of a gene family whose members are implicated in both immune signaling as well as symbiotic signaling. Several data indicate that both LCO's and short chain COs can suppress immune responses. For example, it was reported that symbiotic LCOs and short-chain COs are able to suppress chitin- and flg22-triggered immune responses in the non-host Arabidopsis (Liang et al., 2013). Also, LCO pre-treatment was shown to suppress ROS production induced by an Aphanomyces euteiches culture filtrate in Medicago, although it was not sufficient to block other immune responses (Rey et al., 2018). How symbiotic signaling interacts with plant immunity remains elusive. However, there are several possibilities. For instance, LCO or short-chain CO perception may use the same co-receptors as fungal MAMPs such as chitin and thereby compete for receptor recruitment. In rice, chitin is perceived by a complex of the LysM receptor OsCERK1 and OsCEBiP to trigger downstream signaling. While rice cebip mutants are only impaired in chitin-triggered immunity, cerk1 mutants show defect in both chitin-triggered immunity and mycorrhization, indicating that OsCERK1 plays a dual role in both immune and symbiotic signaling (Zhang et al., 2015a; Miyata et al., 2014). Therefore, LCOs and short-chain COs may outcompete long-chain COs to recruit CERK1 in a symbiotic complex, and thereby interfere with immune responses. Second, ligand perception can also lead to the endocytosis and turn-over of co-receptors, by which defense signaling is attenuated. In addition to direct effects of LCOs/COs on the receptor complexes, interference with immune responses may also occur more downstream. It has been shown that symbiotic signaling affects hormone balances in the root (Liao et al., 2018). Nearly all plant 


\section{CHAPTER 6}

hormones have been implicated in AM symbiosis, including the major defense hormones salicylic acid (SA), jasmonic acid and ethylene, so it is possible that symbiotic signaling affects immune responses through hormonal cross-talk (Limpens et al., 2015). In line with this, it has for example been shown that SA levels in the roots of tomato depend on the compatibility of the AM fungal species (López-Ráez et al., 2010). A continued raise in SA levels in case of an incompatible interaction may in part be due to a deficiency in the activation of the common symbiosis signaling pathway, as studies with CCaMK mutants in pea have suggested that repression of SA levels requires CCaMK activation (Blilou et al., 1999).

Given the potential role of LCOs to suppress host immune responses it is striking that there are currently no indications that pathogenic fungi make use of these molecules for pathogenicity (Huisman et al., 2015). This suggests that interference with immune responses may not be the original function of LCOs as suggested by Liang et al. (2013), but that many of the defense-suppressive effects are indirect.

\section{Effectors}

Effectors have long been known to play a crucial role in pathogenic plant-microbe interactions. Pathogens secrete effectors for example to suppress host immunity, to manipulate host metabolism or to change host cells to facilitate infection (Giraldo et al., 2013; Chaudhary et al., 2014; Lo Presti et al., 2015; Doehlemann et al., 2014). It recently became clear that also symbionts of plants utilize effectors to achieve successful symbioses (Chapter 1 ). The first described effector protein from AM fungi was reported in 2011 by Kloppholz and colleagues. This effector from $R$. irregularis, called SP7, was shown to translocate to the host nucleus and to interact with an ERF19 transcription factor to suppress plant immunity and to maintain a biotrophic interaction (Kloppholz et al., 2011). Our stage-dependent secretome analyses indicated that SP7 is mainly expressed in the extraradical mycelium upon epidermal contact, after which it is rapidly downregulated when the fungus has penetrated the root (Chapter 2). This indicates that the proposed role in suppression of ERF19-mediated immune responses will be limited to the epidermis. It also suggests that other effectors may be required during the intraradical stages of the interaction. A recent study predicted that SP7 has different 
affinities for ERF19 orthologs in different plant species and it was proposed that SP7 may contribute to AM symbiosis in a host-dependent manner (Prasad Singh et al., 2019). In line with this, we observed that SP7 was much higher induced in Chives and Medicago compared to Nicotiana (Chapter2, Table S4).

Recently, it was further suggested that repetitive effector proteins such as SP7 may in fact be processed in the fungus into small effector peptides because potential KEX2 protease cleavage sites were identified in several putative effector proteins including SP7 (Kamel et al., 2017). Effector processing by KEX2 is known to be used by the maize smut fungus Ustilago maydis to generate peptides that regulate pathogenesis (Mueller et al., 2008; Müller et al., 2008). But evidence for processing of SP7 is currently lacking. Nevertheless, the involvement of KEX2 in AM symbiosis was recently shown. AM fungi were shown to produce CLAVATA3 (CLV3)/EMBRYO SURROUNDING REGION-related (CLE) proteins that can be further processed by KEX2 to generate mature CLE peptides (Le Marquer et al., 2019). CLE peptides are a family of plant peptide hormones which regulate a broad aspect of biological programs including shoot meristem development, vascular development, lateral root emergence and systemic signaling of nitrogen status or the rhizobium-legume symbiosis (Yamaguchi et al., 2016). In line with the hormonal role of these CLE peptides, application of synthetic AM fungal CLE peptides affected Medicago root architecture, by stimulating lateral root development and inhibiting primary root growth (Le Marquer et al., 2019). RiCLE1 (RirG208980) showed induced expression across all studied hosts (Chapter2, Table S4), suggesting a conserved function in multiple hosts. However strikingly, we did not see RiCLE significantly induced inside the plant in our analyses (Chapter2, table S6), suggesting that RiCLE may either function transiently or function in stages that are missing from our analyses.

Genomic analyses revealed several effectors containing a LXLFLAK domain in R. irregularis (Lin et al., 2014a; Voß et al., 2018). Effectors carrying LxLFLAK domains, also known as Crinkler (CRN) effectors, were widely described in oomycetes such as Phytophthora (Schornack et al., 2010; Stassen and Van den Ackerveken, 2011). The N-terminal CRN domain was shown to be required for the translocation of CRNs into plant cells (Schornack et al., 2010). There, CRNs can regulate diverse 


\section{CHAPTER 6}

biological processes such as phosphorylation or host transcription to facilitate pathogenesis (van Damme et al., 2012; Song et al., 2015). Interestingly, AM fungi also use CRNs to regulate the symbiosis (Voß et al., 2018). Host-induced gene silencing of RiCRN1 reduced fungal colonization and arbuscule abundance. Strikingly, ectopic expression of RiCRN1 also led to defects in arbuscule development, coinciding with low expression of arbuscule-specific marker genes. These results suggest that the expression level of RiCRN1 should be tightly regulated, possibly at different stages of the interaction. Unfortunately, RiCRN1 is absent from the $R$. irregularis genome version that we used for this thesis (Lin et al., 2014a). However, RiCRN1 was partially annotated as a gene in the genome of Tisserant et al., 2013 (jgi.p|Gloin1|334513). RNAseq analysis to that genome assembly showed highest expression for this gene in germinating spores, and only very low expression in the intraradical stages, suggesting that RiCRN1 functions in early stages of the interaction, and/or that lower intraradical expression levels are important maintain proper arbuscule development. Such levels may differ between hosts as we observed that RiCRN1 was expressed about 2-fold higher in Chives compared to Medicago and Nicotiana. Although we made a significant effort to verify the gene models to annotate effector proteins in Chapter 2 , such annotations may still be improved by the continuing efforts to get better genome assemblies (Chen et al., 2018; Maeda et al., 2018). In this respect it would be highly recommendable that a consensus annotation of the $R$. irregularis genome would be used by the AM research community.

Another AM effector that was functionally implicated in the symbiosis is RiSIS1 (STRIGOLACTONE-INDUCED PUTATIVE SECRETED PROTEIN 1, Tsuzuki et al., 2016). RiSIS1 was originally identified as being induced in germinating spores treated with the synthetic strigolactone GR24 (Tsuzuki et al., 2016). Strigolactones are well-known for their roles as plant growth regulators and stimulants for germination of parasitic plants as well as stimulants of hyphal branching of AM fungi (Waters et al., 2017; Akiyama et al., 2005). Our stage-dependent secretome indicates that RiSIS1 is most highly expressed in intraradical hyphae, to a lesser extent in arbuscules, and the least in extraradical mycelium or germinating spores (Chapter 2, Table S4 and S6). This may suggest that the fungus perceives higher levels of strigolactones when inside the root. Host-induced gene silencing of RiSIS1 significantly reduced hyphal 
density and arbuscule abundance, suggesting that SIS1 plays a key role in the symbiosis (Tsuzuki et al., 2016).

In addition to RiSIS1, we and others identified a putative effector carrying a single LysM domain, named RiSLM, that was one of the highest expressed effectors in the symbiosis with a wide range of host plants and was also shown to be induced by GR24 (Chapter 2 and 4; Tsuzuki et al., 2016; Schmitz et al., 2018). Interestingly, the expression profiles of RiSLM and RiSIS1 are highly correlated (Spearman correlation=0.92) across 101 transcriptome data collected from different hosts/growing conditions/ tissue types either from in-house or public database (data not shown). Like RiSIS1, RiSLM also showed the highest expression in intraradical mycelium. Therefore it is tempting to speculate that they may be coregulated by the same transcription factor(s). Secreted LysM effectors are well known from fungal pathogens where they play important roles to evade chitin-triggered plant immunity. Such LysM effectors can protect fungal cell walls from the action of plant chitinases, and/or bind fungal cell wall derived COs to prevent induction of plant immune responses (Marshall et al., 2011; Mentlak et al., 2012; Lee et al., 2014; Takahara et al., 2016; Kombrink et al., 2017). In Chapter 4, we performed functional studies on RiSLM. Our results indicate that RiSLM is able to protect fungal cell walls against chitinases, and that it can also bind COs (particularly long-chain $\mathrm{CO}$ such as $\mathrm{CO} 7$ and $\mathrm{CO} 8$ ) to evade chitin-triggered plant immunity. However, the binding affinity between RiSLM and CO7 (9 $\mu \mathrm{M})$ is significantly lower than the affinity reported for the pathogenic LysM effector ECP6 (280 pM) and only slightly higher than the reported affinity of plant chitin receptors such as LjLYS6 $(26 \mu \mathrm{M})$ (Bozsoki et al., 2017; de Jonge et al., 2010). This suggests that RiSLM does not just sequester $\mathrm{CO}$ to prevent them from binding to their receptors. It was hypothesized fungal LysM effectors might also directly interact with plant chitin-receptors to interfere with receptor dimerization to impair chitin-triggered immunity. This was for example postulated based on the observation that only the second LySM domain of ECP6 is sufficient to suppress chitin-triggered immunity although it has much lower chitin-binding affinity (micromolar range) compared to the other two LysM domain (picomolar range). A preliminary experiment suggested that RiSLM can indeed interact with the (co)receptor MtLYR4 in a chitin-dependent manner (Limpens, unpublished data). However, additional experimentation is required to 


\section{CHAPTER 6}

solidify this claim. It is intriguing that RiSLM can bind to myc-LCOs with higher affinities than that for CO7 (Chapter 4). Myc-LCOs can be either sulfated on the reducing GICNAc residue or remain non-sulfated. Although binding of sulfated myc-LCOs by RiSLM did not inhibit symbiotic signaling, binding of non-sulfated LCOs severely impaired symbiotic gene induction in Medicago. It is known that Medicago as a legume, has evolved LysMRLKs that have a very high affinity for sulfated LCOs that are secreted by its rhizobial symbiont Sinorhizobium meliloti, while it is much less responsive to non-sulfated rhizobial LCOs (Philippe Roche et al., 1991; Lerouge et al., 1990). Myc-LCOs and rhizobial Nod factors of S. meliloti are very similar in structure (Maillet et al., 2011). Therefore the difference in affinity of the Medicago receptors for sulfated and non-sulfated LCOs may explain the different effects of RiSLM. However, since it has been shown that sulfated and non-sulfated myc-LCO induce different transcriptional responses in Medicago (Czaja et al., 2012) it cannot be ruled out that RiSLM affects symbiotic responses during colonization. A direct role for RiSLM in symbiotic signaling is also suggested by the higher induction of symbiotic marker genes upon application of equimolar amounts of RiSLM and CO4 compared to CO4-only treatment in Medicago roots. It is potentially related to the various LySM-RLK receptor complexes involved as discussed above. This remains an interesting topic to be studied in the future.

Decreased RiSLM expression in arbuscules compared to intraradical mycelium suggests that either arbuscule-containing cells show a low capacity for chitin-triggered immunity, or that AM fungi have an alternative strategy to suppress plant immunity in arbuscule cells (e.g. using different effectors). Based on our transcriptome data, we identified a set of effectors highly expressed specifically in arbuscule cells (Chapter 2). Such effectors may play essential roles to suppress host immunity or to support arbuscule development, maintenance or function. Intriguingly, we also found three Medicago secreted LysM proteins/effectors that were highly and specifically expressed in arbuscule-containing cells. Phylogenetic analyses suggest those LysM effectors are conserved in AM host plants (Bai, Roswanjaya, Limpens, unpublished data). Surprisingly, those plant LysM effectors did not show a function in chitin-triggered plant immunity or symbiosis (Bai, Roswanjaya, Limpens, unpublished data). Further investigation is needed to unravel the function of these 
arbuscule-specific plant LysM effectors. Among the arbuscule-specific fungal effector candidates, 14 nuclear-targeting effectors were predicted based on the presence of a plant nuclear localization signal. By transient expression in Nicotiana leaves, we observed that those effectors could indeed localize to the plant nucleus, with some even showing more specific accumulation in the nucleolus and subnuclear bodies. Nuclear targeting may suggest a function as transcriptional regulators or chromatin modifiers in arbuscule-containing plant cells. However, evidence for host-translocation in arbuscule-containing cells is currently lacking. Furthermore, how fungal effectors can enter the cells still remains unclear (Petre and Kamoun, 2014; Lo Presti and Kahmann, 2017). Fungi do not harbor effector delivery systems such as the bacterial type III secretion system, which is used to deliver bacterial effectors to the host cell. It was reported that some fungi or oomycete effector proteins contain motifs such as RxLR to mediate effector endocytosis by binding to cell membrane phosphatidylinositol-3-phosphate (Lo Presti and Kahmann, 2017; Dou et al., 2008; Kale et al., 2010). This mechanism was also proposed for MiSSP7, as MiSSP7 contains a RxLR-like motif that also binds phosphatidylinositol-3-phosphate (Plett et al., 2011). However, a recent study indicated that the RxLR domain in Phytophthora infestans AVR3a is cleaved before secretion (Wawra et al., 2017), suggesting a role for the RxLR motif in effector secretion rather than host entry. Therefore, the role of RxLR motif in host entry remains controversial. We did not detect a common signature/domain in the putative $R$. irregularis effectors that may mediate host translocation. Recent studies revealed extensive vesicular structures in the peri-arbuscular space (Ivanov et al., 2019; Roth et al., 2019). Similar structures were observed in the interface between pathogenic fungal haustoria and plant cells (Lo Presti and Kahmann, 2017). In the biotrophic interaction of Magnaporthe oryzae and rice, it was shown that effectors can also be delivered to the host cell via a process called unconventional secretion, which may involve the fusion of fungal exosomes to the host cells (Khang et al., 2010; Giraldo et al., 2013). Also Phytophthora sojae and Verticillium dahliae were shown to secrete isochorismatases, which lack conventional signal peptides, in an unconventional manner to disrupt salicylate-mediated innate immunity (Liu et al., 2014). In this thesis, I only focused on effectors carrying a signal peptide. More investigations are required in the future to study unconventionally secreted effectors of AM fungi. 


\section{CHAPTER 6}

The role of extracellular vesicles is gaining interest in plant-microbe interactions (Rutter and Innes, 2018). A recent study revealed an essential role of extracellular vesicles to deliver small RNAs from the plant to the fungal pathogen Botrytis cinerea to silence fungal virulence genes (Cai et al., 2018). Conversely, Botrytis cinerea was also shown to produce small RNAs which can translocate to the plant host to silence host immunity genes (Weiberg et al., 2013). A similar mechanism was recently proposed in the rhizobium symbiosis, as rhizobial tRNA-derived small RNAs were shown to regulate host gene expression to facilitate the symbiosis (Ren et al., 2019). Therefore, the current concept of effectors should be extended to include (small) RNAs. It would be intriguing to investigate whether AM fungi and their hosts also utilize small RNAs and RNA silencing machineries to regulate symbiosis-related genes to facilitate the symbiosis. The ability of HIGS to silence AM fungal genes together with the observation of numerous extracellular membrane structures in the AM symbiosis supports such a possibility.

Recently, it was proposed that effectors may also be used in the interaction of fungi with other microbes in the soil (Snelders et al., 2018). Therefore it is possible that, besides an interaction with the host, AM fungi secrete effectors to inhibit pathogenic microbes or to promote beneficial microbes. This may eventually lead to an optimal endophytic/rhizosphere/soil microbiome that favors plant growth. It was shown that AM fungi directly interact with soil microbiome such as phosphate-solubilizing bacteria to stabilize the symbiosis (Zhang et al., 2016). In addition, AM hyphal exudates can stimulate phosphatase gene expression in the phosphatesolubilizing bacteria Rahnella aquatilis (Zhang et al., 2018).

Host-dependent effectors

Fungal effectors are important determinants of the host-range of pathogenic fungi. Because AM fungi also utilize effectors to control the symbiosis, it raised the question whether AM fungi secrete effectors in a host-dependent manner to establish symbiosis with different hosts. To address this question, we performed transcriptome profiling of the AM secretome in the three phylogenetically distant plant hosts Medicago, Chives and Nicotiana (Chapter 2). Our results showed that the majority of secreted protein (SP)-encoding genes are commonly expressed in all hosts. However, on top of this common set of $\sim 300$ SPs, $\sim 15 \%$ of 
the SPs were expressed in a host-dependent manner (Chapter 2). This suggested that AM fungi perceive the (local) host environment and adjust their secretome accordingly. Most host-dependent SPs were especially expressed during the intraradical stages in Medicago. Many of these putative effectors lacked known protein domains. However, some of them show similarity to known effectors or contain functional domains. One of these effectors, RirG091260 was much higher expressed in Chives compared to Medicago and Nicotiana and showed homology to the $\beta$-glucan binding effector FGB1 of the beneficial endophyte Piriformospora indica. PiFGB1 was shown to bind $\beta$-glucan to interfere with $\beta$-glucaninduced plant immunity and to influence cell wall properties to facilitate colonization (Wawra et al., 2016). In line with this, FGB1-like RirG091260 was also able to bind to $\beta$-glucan (curdlan) and to impair laminarininduced plant immune responses (Wang et al., unpublished data). The Chives-dependent expression of RirG091260 raises the question whether this is related to a difference in $\beta$-glucan perception in different plants. However, preliminary follow up experiments in the group of Limpens, have shown that RirG091260 can also interfere with chitin and flg22-triggered immune responses (Wang et al., unpublished). Therefore, RirG091260 may be more generally involved in the suppression of PAMP-triggered immunity, possibly by binding to glycan-groups of the highly glycosylated PAMP receptors in plants. It has been shown that correct glycosylation plays an important role in the activity of such receptors (Häweker et al., 2010).

In addition to suppressing host immunity, AM effectors may also affect host or fungal metabolism. It is known that Phytophthora and Pythium secrete sterol/lipid-binding effectors, also known as elicitins, to take up sterols/lipids (Derevnina et al., 2016; Blein et al., 2002). Although no elicitins were detected in AM genomes, we noticed several potential lipid binding effectors (MD-2-related lipid-recognition (ML) domain containing) and lipase-like effectors to be expressed in a host-dependent manner. It will be interesting to study whether these function as lipid/sterol shuttles to take up fatty acids by AM fungi.

\section{Secondary metabolism}

To maintain a mutually beneficial symbiosis in a wide-range of hosts AM fungi must be able to efficiently adapt their metabolism to the local 


\section{CHAPTER 6}

environment of the host. Upon microbial colonization, plants rewire their metabolic pathways to create an unfavorable local environment to enhance resistance against potential pathogens. For instance, plants may locally produce reactive oxygen species or synthesize antifungal compounds to inhibit fungal growth. In addition, nutrients are tightly controlled by the hosts to limit pathogen growth. Therefore, AM fungi need to have hostadaptation or host-detoxifying machineries to successfully colonize such a broad-range of hosts.

Plants can produce numerous antimicrobial compounds, named phytoalexins, that differ between plant species (Pedras and Ahiahonu, 2005). Therefore, AM fungi likely need to be able to evade the production of, or detoxify, such antifungal phytoalexins. The ability to detoxify host phytoalexins was shown before to control the host-range of fungal pathogens. For example, upon fungal colonization, plant hosts such as tomato produce the saponin tomatine to inhibit fungal growth. In turn, fungal pathogens such as Fusarium oxysporum and Cladosporium fulvum can produce saponin detoxifying enzymes (tomatinase) to convert tomatine into less toxic compounds to maintain full fungal virulence (Pareja-Jaime et al., 2008; de Wit et al., 2012). However, the closely related Dothistroma septosporum which does not produce such enzymes fails to colonize tomato (Ökmen et al., 2013). Also, Gaeumannomyces graminis mutants that are not able to detoxify host saponins are only able to colonize hosts such as wheat that do not produce saponin, but not hosts such as oat that do (Bowyer et al., 1995). Although AM fungi are able to colonize both saponin-producing and non-producing plants, we did not detect obvious saponin-detoxifying enzymes in the $R$. irregularis genome.

One of the most common plant defense mechanisms is the production of reactive oxygen species (ROS). Therefore, ROS detoxifying mechanisms are essential for microbial colonization. We noticed that genes related to oxido-reductive processes are induced when AM fungi colonize plants (Chapter 3 ). These include many genes related to secondary metabolism such as cytochrome P450s (Chapter 3). The expression of different cytochrome $\mathrm{P} 450$ genes in different hosts might point to different detoxifying mechanisms in different hosts. Fungi have developed efficient antioxidant mechanism, such as glutathione, thioredoxins, superoxide dismutases and catalases (SODs) (Montibus et al., 2015). It was reported 
that a symbiosis-induced copper-zinc superoxide dismutase (SOD) gene from $R$. irregularis is undergoing rapid evolution and diversifying selection (Corradi et al., 2009), suggesting that antioxidant mechanisms might be crucial for AM symbiosis. A Gigaspora margarita SOD gene was shown to be induced in planta and likely functions as an anti-oxidant enzyme in intraradical mycelium and arbuscules (Lanfranco et al., 2005). In line with this, we observed high expression of a $\mathrm{Cu} / \mathrm{Zn}$ SOD gene in arbuscules and intraradical mycelium (Chapter 3 ). SODs require extensive supply of zinc or copper, which explains the high expression of copper and zinc transporter genes in planta (Chapter 3). We detected higher expression of fungal glutathione biosynthesis-related genes in colonized Chives and Nicotiana compared to Medicago (Chapter 3). This suggests that AM fungi experience different levels of ROS, possibly as a result of varying levels of defense responses. Alternatively, it could be related to differences in metabolic activity in the different hosts, as electron transfer reactions during secondary metabolism are known to generate ROS.

So far, secondary metabolites are poorly studied in AM fungi. However, they were proposed to be crucial in plant-microbe interactions as they can act as virulence factors and affect growth of the hosts (Macheleidt et al., 2016). Several fungi have been shown to produce phytohormones to manipulate their host plants (Chanclud and Morel, 2016). For example, the fungal pathogen $F$. fujikuroi can secrete secondary metabolites such as gibberellins to induce rapid growth of their hosts (Malonek et al., 2005). Also symbionts such as ectomycorrhiza or Piriformospora indica have been suggested to produce phytohormones (Chanclud and Morel, 2016). Whether AM fungi produce phytohormones has been largely understudied (Barea and Azcón-Aguilar, 1982).

\section{Iron and immunity}

Inside the host, the levels of essential metals such as iron are tightly controlled by the hosts to inhibit microbial growth (Braunsdorf et al., 2016). Therefore, microbes have invented metal acquisition strategies to ensure sufficient nutrient supply. For example, a high affinity iron permease expressed in intraradical hyphae was reported to be essential for pathogenicity of Ustilago maydis (Eichhorn et al., 2006). Further, it was proposed that plants can target the bacterial iron acquisition system to inhibit bacterial growth (Nobori et al., 2018). We observed 


\section{CHAPTER 6}

that oxidation and reduction processes were enriched in all hosts in an arbuscule-dependent manner (Chapter 3 ). This is likely due to active secondary metabolism as many cytochrome P450 genes were found in those gene sets. This suggests that AM fungi may need high demand of iron, particularly in arbuscule cells, to support proper function of cytochrome P450s (Chapter 3). Interestingly, observed that two $R$. irregularis iron transporters were expressed in a "dicot"- and arbusculedependent manner. In contrast, all major Medicago iron transporters IRTs are downregulated in arbuscule-containing cells compared to surrounding cortex cells. This suggests that AM fungi absorb iron from the periarbuscular space to support the high iron demand in arbuscules, although experimental evidence is currently lacking. In fact, the membrane iron transporter jgi_p_Gloin1_347887 represents one of the most highly expressed genes in arbuscules. Some studies have suggested that AM fungi can also deliver iron to the plant, although this seems to be host and/or condition dependent (Caris et al., 1998). In addition to the iron transporter, we observed that a IucA / IucC gene (RirG008590), which may regulate siderophore biosynthesis, was higher expressed in the two dicots Medicago and Nicotiana compared to the monocot Chives and in an arbuscule-dependent manner. Siderophores are secondary metabolites which can chelate iron to facilitate iron uptake or stabilize free iron levels to avoid iron toxicity. Pathogenic fungi use siderophores to compete for iron uptake with their host plants or to enhance their resistance to oxidative stress. It has been shown that a lack of siderophore biosynthetic genes in pathogenic fungi impaired pathogenicity, as well as symbiosis by the beneficial endophyte Epichloe festucae (Johnson, 2008; Oide et al., 2006; Verbon et al., 2017; Johnson et al., 2013). Dicot-dependent induction of genes involved in iron transport and homeostasis suggests that AM fungi might be more iron-deficient in Medicago and Nicotiana compared to Chives.

\section{Reciprocal nutrient exchange}

A stable AM symbiosis requires the reciprocal exchange of nutrients, which is considered to occur mainly at the arbuscule interface (Chapter 1). Interestingly, in Medicago it was shown that both plant and fungus can monitor the benefits that they obtain from each other and adjust 
the transfer of nutrients accordingly (Kiers et al., 2011). So, fungi that provided more $\mathrm{P}$ to their host were rewarded with more carbon, and vice versa if the plant provided more carbon it obtained more $P$ from the fungus. How such reciprocal rewarding, as part of a biological market theory (Werner et al., 2014) is controlled is still largely unknown. It requires more detailed insight into the molecular components involved in both plant and fungus.

It has become clear that the benefits obtained from the fungus extend beyond mere phosphate delivery. For example, the early degradation of arbuscules in the symbiotic phosphate transport mutant Mtpt4 could be rescued by growing plants under nitrogen starved conditions. This required the action of the MtAMT2;3 ammonium transporter, suggesting that the fungus provides ammonium which is sensed by the transceptor MtAMT2;3 to control arbuscule maintenance (Breuillin-Sessoms et al., 2015). Similar to the effect of host carbon supply on phosphate delivery by the fungus, it was shown that carbon supply also controls the nitrogen flux from the fungus (Fellbaum et al., 2012). In addition to phosphate and nitrogen, AM fungi provide water and other micronutrients to their host. In Chapter 3 we highlight several fungal transporters that are induced especially in the arbuscules, and are therefore key candidates to control the transfer of these nutrients from arbuscules to the host. We further noticed the induction of several amino acid/peptide transporters in the symbiosis. In the rhizobium nodule symbiosis an amino acid cycle has been shown to play a role in the supply of fixed nitrogen to the legume hosts (Day et al., 2001; Lodwig et al., 2003; White et al., 2007). It will therefore also be interesting to study the role of amino acid exchange in the AM symbiosis. Work by Kikuchi et al. (2016) has implicated the action of an aquaporin of Rhizophagus clarus (RCAQP3) in the long distance transport of poly-P and proposed a model for water potential gradients at the arbuscules to control $\mathrm{P}$ delivery to the host. In this model, a water flow from fungus to plant, controlled by host transpiration, facilitates the flow of poly-P from the extraradical mycelium to the arbuscules, where it is processed into $\mathrm{Pi}$ to be transported to the host. In $R$. irregularis, the RCAQP3 ortholog RiAQPF2 (RirG028370) was highly expressed through all developmental stages, which is in line with its function. However, we noticed that it was induced stronger in Chives (1.87-fold) and Medicago (2.12-fold) compared to Nicotiana, possibly related to different 


\section{CHAPTER 6}

transpiration rates or water use efficiency of the various hosts.

How plants monitor the amount of nutrients received is still poorly understood. Signaling functions of potential phosphate or ammonium transceptors may play a role locally and long distance transport of peptide signals, for example by CLE and CEP peptides (Bisseling and Scheres, 2014; Taleski et al., 2018) may be used systemically. Recently, it was found that the degradation of arbuscules requires the action of the MtMYB1 transcription factor (Floss et al., 2017), which is highly expressed specifically in arbuscule-containing cells. MtMYB1 controls the expression of numerous hydrolases thought to be involved in arbusucle degradation. Interestingly, MtMYB1 is already highly expressed in healthy, non-degrading arbuscules, suggesting that its activity is posttranscriptionally regulated. MtMYB1 was shown to interact with both DELLA proteins and MtNSP1, which are both implicated in the symbiotic signaling pathway (Floss et al., 2017; Pimprikar and Gutjahr, 2018) and required for full MtMYB1 activity. The promotional effects of DELLA proteins on MtMYB1 activity has led to the suggestion that local GA levels may influence arbuscule life time (Takeda et al., 2015; Pimprikar et al., 2016) Alternatively, additional regulators may be involved. For example, we noticed the high expression of two SPX-domain proteins in our arbuscule-specific transcriptome data. The SPX domain is thought to function as a phosphate sensor and SPX proteins are known to suppress the phosphate-starvation response by inhibiting the action of the key myb transcription factor PHR1 in a phosphate-dependent manner (Puga et al., 2014; Wang et al., 2014b). Therefore it will be interesting to test whether the arbuscule-induced SPX proteins function to monitor $\mathrm{P}$ supply by the fungus to control for example the activity of MtMYB1 or other proteins.

In return for the nutrients provided to the plant, AM fungi obtain photosynthates as rewards. Originally it was thought that the fungi mainly obtain sugars (hexoses) as carbon source from the plant (Solaiman and Saito, 1997; Shachar-Hill et al., 1995; Bago et al., 2003; Bago et al., 2000). However, with the availability of the $R$. irregularis genome sequence, it became clear that AM fungi lack a key enzyme (type I fatty acid synthase, FasI) to produce fatty acids (Wewer et al., 2014). This makes AM fungi fatty acid auxotrophs that rely on their plant hosts to supply fatty acid precursors (Chapter 1 ). 
Fatty acids can be converted into sugars in the fungus and may thus serve as a major nutritional carbon source for the fungus. This is further supported by the recent finding that application of the proper fatty acids allowed $R$. irregularis to grow a-symbiotically and even to make viable spores, although the spores were smaller than spores formed in symbiosis (Kameoka et al., 2019; Sugiura et al., 2019). The work by Kameoka and Sugiura and colleagues further suggested that different fungi may require different fatty acid precursors. In line with this we observed substantial variation in the expression of fatty acid metabolic enzymes in three different host species (Chapter 3 ). This leads us to speculate that variation in fatty acid metabolism in plant and fungus may contribute to observed host preferences found in nature.

The key role of fatty acids in AM symbiosis raises the question what the contribution of sugars provided by the plant is in terms of nourishment for the fungus. On the fungal side a monosaccharide transporter, called RiMST2 (RirG108040), has been identified and shown to be required for proper arbuscule development (Helber et al., 2011). It could transport glucose as well as cell wall derived monosaccharides. Previous isotope tracer experiments indicated that glucose is the main hexose taken up by the fungus (Bago et al., 2000). We identified an additional MST2 family member (RirG088730) that, like RiMST2, is most highly expressed in arbuscules. This suggests that arbuscules are a major place where glucose uptake takes place, although additional expression in intraradical mycelium points to the potential uptake of monosaccharides at those places as well. To identify plant components involved in the transfer of glucose across the PAM, we searched for SWEET sugar transporters in our arbuscule-specific transcriptome data, as these are known to function as sugar exporters in plants (Chen et al., 2010). This identified one SWEET transporter, MtSWEET1b, that was especially highly expressed in the arbuscule-containing cells (Chapter 5 ). Multiple SWEET family members changed in expression upon mycorrhization, similar to what was reported in potato (Manck-Götzenberger and Requena, 2016), however only MtSWEET1b showed induction in cells that are in contact with fungal hyphae. MtSWEET1b localized to the PAM and specifically transported glucose in a yeast transport assay. Given the sink strength of the fungus it is most likely that MtSWEET1b exports glucose into the peri-arbuscular space where it is taken up with the help of MST2 transporters into the 


\section{CHAPTER 6}

fungus. Excess sugars can subsequently be converted into glycogen to be stored and transported in the fungus. The identification of MtSWEET1b allowed us to test its role in symbiosis. Arbuscule-specific overexpression of MtSWEET1b increased fungal colonization levels in the root. Strong arbuscule-specific expression of dominant-negative MtSWEET1b mutant proteins caused a more early collapse of arbuscules, which correlated with a lower MtPT4 expression level. Strikingly, we did not observe any defect in mycorrhization in two $m$ tsweet $1 b$ Tnt1-retrotransposon insertion mutants. Since SWEET proteins are known to function as (hetero) oligomers (Xuan et al., 2013), it is likely that additional SWEET members may take over the function of MtSWEET1b. Intriguingly, preliminary follow-up experiments indicated a differential regulation of MtSWEET1b and MtSTR/STR2 when Medicago roots were colonized by the cooperative AM fungus $R$. irregularis or the less-cooperative Glomus aggregatum (Kiers et al., 2011). The ratio of MtSWEET1b to MtSTR expression was higher with the cooperative strain than with the less-cooperative strain (An and Limpens, unpublished). It may suggest that MtSWEET1b is involved in the reciprocal rewarding in response to higher $\mathrm{P}$ supply by the fungus. On the other hand, the observed effects on arbuscule maintenance could also point to an additional role for MtSWEET1b that is unrelated to glucose transport. The Arabidopsis AtSWEET13 and AtSWEET14 proteins were recently shown to be able to transport GA (Kanno et al., 2016). Regulation of GA signaling is particularly essential for arbuscule formation as DELLA proteins, key transcriptional repressors in GA signaling, are essential for arbuscule formation and play a role in arbuscule degradation (Floss et al., 2017; Floss et al., 2013; Takeda et al., 2015). GA perception leads to the degradation of DELLA proteins, which is therefore expected to influence arbuscule development. Preliminary data indicate that MtSWEET1b can indeed transport GA in a yeast assay (Seo and Limpens, unpublished data). Although this clearly needs to be studied further, it could be envisioned that MtSWEET1b controls GA levels in arbuscule-containing cells, which in turn influences arbuscule life-time through MtMYB1 regulated gene expression. Hopefully, future work will shed more light on the complex yet key process of reciprocal nutrient exchange in AM symbiosis. 


\section{Future perspectives}

The availability of a first AM fungal genome sequence has opened up many opportunities to get better insight into the fungal aspects of this symbiosis. Along with the development of new sequencing technology, especially long-read sequencing such as Pacbio technology or Nanopore technology, AM fungal genome assemblies are continuing to be improved (Chen et al., 2018; Maeda et al., 2018). This will greatly facilitate our insight into genetic diversity within AM fungi and allow a better annotation of fungal genomes. Through Pacbio sequencing, it was found that $R$. irregularis lacks the typical tandem repeat structure of ribosomal DNA. As a result, the scattered rDNA sequences are very heterogeneous in contrast to other eukaryotic rDNA sequences (Maeda et al., 2018). It was proposed that this special structure of rDNAs may facilitate AM fungi to adapt to different hosts as it may result in diverse ribosomes that differentially affect translation (Maeda et al., 2018).

Most transcriptome profiling studies on AM symbiosis have used whole root samples. A recent RNAseq study by Kameoka and colleagues (Kameoka, Maeda, et al., 2019) focused mostly on various extraradical stages, such as germ tubes, runner hyphae, branched absorbing structures, immature spores, and mature spores. Whole root samples have the drawback that they lack detail about the different plant cell-types and intraradical fungal stages. Although several publications reported cell-type specific transcriptomic analyses of AM symbiosis (Gaude, Bortfeld, et al., 2012; Hogekamp and Küster, 2013; Hogekamp et al., 2011), those studies used gene chips, which only included a very limited number of fungal genes. In this thesis, I applied laser microdissection in combination with low input RNASeq to study transcriptomes in arbuscule-containing or hyphae-containing cells, both from the plant and the fungal side. This strategy allowed us to successfully study plant and fungal transcriptomes simultaneously and discover novel regulators. Nevertheless, application of laser microdissection largely relies on the morphological differences of different cell types. There are AM stages which are less easy to distinguish based on morphology. For example, it would be tricky to collect cell types with minor morphological differences, such as developing arbuscules and degrading arbuscules. A promising approach to get a higher resolution of such stages would be to apply novel single cell (or nucleus) RNA sequencing strategies (Ryu et al., 2019; Denyer et al., 2019). 


\section{CHAPTER 6}

A major challenge for the future will be to study the functional role of many of the identified fungal genes. This is especially difficult since it is currently not possible to stably transform the fungus, likely due to its extreme coenocytic nature (Helber and Requena, 2007). Most studies on fungal genes rely on host-based techniques such as host-induced gene silencing (HIGS) or virus-induced gene silencing (VIGS). Although those techniques were successfully used to study fungal genes, they rely on the hosts to deliver dsRNAs and/or small interfere RNAs to the fungal side to generate silencing. Therefore, it is impossible to use these techniques to study AM fungal genes implicated in pre-symbiotic development. In addition, a lot of variation in the efficiency of HIGS or VIGS has been reported and some genes seem to be recalcitrant to be silenced. Whether this indicates that only certain RNA structures can be delivered to the fungal side remains unclear. Recent studies found that viruses naturally exist in AM fungi (Turina et al., 2018; Neupane et al., 2018). Therefore, a potential strategy to functionally study AM genes might be to design viral vectors for transfection in $A M$ fungi. Other options to investigate could be the use of nanoparticles to deliver (ds)RNA/DNA into the fungus (Cunningham et al., 2018). It has been recently shown that fluorescent nanoparticles are efficiently taken up by $A M$ fungi and can be used to trace phosphate translocation from the fungus to the plant (Whiteside et al., 2019).

A drawback in the use of AM fungi is the inability to culture AMF without their hosts, although usage of hairy root culture enables AM culture in axenic condition (Srinivasan et al., 2014). Inspired by the finding that AM fungi fully rely on plant hosts to supply fatty acids (Wewer et al., 2014; Luginbuehl et al., 2017; Jiang et al., 2017; Keymer et al., 2017), recent studies showed that exogenous application of fatty acids including myristate and palmitoleic acid can stimulate a-symbiotic sporulation (Sugiura et al., 2019; Kameoka, Tsutsui, et al., 2019). Such efforts may provide promising strategies to efficiently culture AM fungi ex planta in the future.

To realize the promise of applying AM fungi as biofertilizers in sustainable agricultural practices, one of the most important issues to address in the future will be to unravel the molecular mechanisms that underlie variation in symbiotic efficiency between different plant-fungal combinations. Various studies indicate an interplay between genetic variation in the plant and 
the fungus (Smith and Read, 2008; Sanders and Croll, 2010; Koch et al., 2017; Watts-Williams et al., 2019; Mateus et al., 2019). Exploiting such natural genetic variation in symbiotic efficiency is therefore envisioned to provide a powerful tool to get insight into this key aspect of this symbiosis in the near future. 


\section{REFERENCES}


Akiyama, K., Matsuzaki, K.I. and Hayashi, H. (2005) Plant sesquiterpenes induce hyphal branching in arbuscular mycorrhizal fungi. Nature, 435, 824-827.

Ané, J.-M., Kiss, G.B., Riely, B.K., et al. (2004) Medicago truncatula DMI1 required for bacterial and fungal symbioses in legumes. Science (80-. )., 303, 1364-1367.

Arrighi, J.-F., Barre, A., Amor, B. Ben, et al. (2006) The Medicago truncatula lysine motif-receptor-like kinase gene Family includes NFP and new nodule-expressed genes. Plant Physiol., 142, 265-279.

Auriac, M.-C. and Timmers, A.C.J. (2007) Nodulation studies in the model legume Medicago truncatula : advantages of using the constitutive EF1a promoter and limitations in detecting fluorescent reporter proteins in nodule tissues. Mol. Plant-Microbe Interact., 20, 1040-1047.

Bago, B., Pfeffer, P. and Shachar-hill, Y. (2000) Update on symbiosis arbuscular mycorrhizas. Plant Physiol., 124, 949-957.

Bago, B., Pfeffer, P.E., Abubaker, J., Jun, J., Allen, J.W., Brouillette, J., Douds, D.D., Lammers, P.J. and Shachar-Hill, Y. (2003) Carbon export from arbuscular mycorrhizal roots involves the translocation of carbohydrate as well as lipid. Plant Physiol., 131, 1496-1507.

Baier, M.C., Keck, M., Gödde, V., Niehaus, K., Küster, H. and Hohnjec, N. (2010) Knockdown of the symbiotic sucrose synthase MtSucS1 affects arbuscule maturation and maintenance in mycorrhizal roots of Medicago truncatula. Plant Physiol., 152, 1000-1014.

Barea, J.M. and Azcón-Aguilar, C. (1982) Production of plant growthregulating substances by the vesicular-arbuscular mycorrhizal fungus Glomus mosseae. Appl. Environ. Microbiol., 43, 810-813.

Belmondo, S., Fiorilli, V., Pérez-Tienda, J., Ferrol, N., Marmeisse, R. and Lanfranco, L. (2014) A dipeptide transporter from the arbuscular mycorrhizal fungus Rhizophagus irregularis is upregulated in the intraradical phase. Front. Plant Sci., 5, 436.

Benedito, V.A., Torres-Jerez, I., Murray, J.D., et al. (2008) A gene expression atlas of the model legume Medicago truncatula. Plant J., 55, 504-513.

Besserer, A., Puech-Pagès, V., Kiefer, P., et al. (2006) Strigolactones stimulate arbuscular mycorrhizal fungi by activating mitochondria. PLOS Biol., 4, 1239-1247.

Bezrutczyk, M., Yang, J., Eom, J.-S., et al. (2018) Sugar flux and signaling in plant-microbe interactions. Plant J., 93, 675-685.

Bi, G. and Zhou, J.-M. (2017) MAP kinase signaling pathways: a hub of plant-microbe interactions. Cell Host Microbe, 21, 270-273.

Bianciotto, V., Barbiero, G. and Bonfante, P. (1995) Analysis of the cell cycle in an arbuscular mycorrhizal fungus by flow cytometry and bromodeoxyuridine labelling. Protoplasma, 188, 161-169.

Bisseling, T. and Scheres, B. (2014) Nutrient computation for root architecture. Science (80-. )., 346, 300-301. 
Blee, K. a. and Anderson, A.J. (1998) Regulation of arbuscule formation by carbon in the plant. Plant J., 16, 523-530.

Blein, J.-P., Coutos-Thévenot, P., Marion, D. and Ponchet, M. (2002) From elicitins to lipid-transfer proteins: a new insight in cell signalling involved in plant defence mechanisms. Trends Plant Sci., 7, 293-296.

Blilou, I., Ocampo, J.A. and Garcia-Garrido, J.M. (1999) Resistance of pea roots to endomycorrhizal fungus or Rhizobium correlates with enhanced levels of endogenous salicylic acid. J. Exp. Bot., 50, 16631668.

Bowyer, P., Clarke, B.B.R., Lunness, P., Daniels, M.J. and Osbourn, A.A.E. (1995) Host range of a plant pathogenic fungus determined by a saponin detoxifying enzyme. Science (80-. )., 267, 371-374.

Bozsoki, Z., Cheng, J., Feng, F., et al. (2017) Receptor-mediated chitin perception in legume roots is functionally separable from Nod factor perception. Proc. Natl. Acad. Sci. U. S. A., 114, E8118-E8127.

Braunsdorf, C., Mailänder-Sánchez, D. and Schaller, M. (2016) Fungal sensing of host environment. Cell. Microbiol., 18, 1188-1200.

Bravo, A., Brands, M., Wewer, V., Dörmann, P. and Harrison, M.J. (2017) Arbuscular mycorrhiza-specific enzymes FatM and RAM2 fine-tune lipid biosynthesis to promote development of arbuscular mycorrhiza. New Phytol., 214, 1631-1645.

Breuillin-Sessoms, F., Floss, D.S., Gomez, S.K., et al. (2015) Suppression of arbuscule degeneration in Medicago truncatula phosphate transporter4 mutants is dependent on the ammonium transporter 2 family protein AMT2;3. Plant Cell, 27, 1352-1366.

Breuillin, F., Schramm, J., Hajirezaei, M., et al. (2010) Phosphate systemically inhibits development of arbuscular mycorrhiza in Petunia hybrida and represses genes involved in mycorrhizal functioning. Plant J., 64, 1002-1017.

Broghammer, a., Krusell, L., Blaise, M., et al. (2012) Legume receptors perceive the rhizobial lipochitin oligosaccharide signal molecules by direct binding. Proc. Natl. Acad. Sci. U. S. A., 109, 13859-13864.

Burg, H.A. van den, Harrison, S.J., Joosten, M.H.A.J., Vervoort, J. and Wit, P.J.G.M. de (2006) Cladosporium fulvum Avr4 protects fungal cell walls against hydrolysis by plant chitinases accumulating during infection. Mol. plant-microbe Interact., 19, 1420-1430.

Cai, Q., Qiao, L., Wang, M., He, B., Lin, F., Palmquist, J., Huang, S.-D. and Jin, H. (2018) Plants send small RNAs in extracellular vesicles to fungal pathogen to silence virulence genes. Science (80-. )., 360, 1126-1129.

Calabrese, S., Pérez-Tienda, J., Ellerbeck, M., et al. (2016) GintAMT3 - a low-affinity ammonium transporter of the arbuscular mycorrhizal Rhizophagus irregularis. Front. Plant Sci., 7, 1-14.

Camps, C., Jardinaud, M.-F., Rengel, D., Carrère, S., Hervé, C., Debellé, F., Gamas, P., Bensmihen, S. and Gough, C. (2015) Combined genetic and transcriptomic analysis reveals three major 
signalling pathways activated by Myc-LCOs in Medicago truncatula. New Phytol., 208, 224-240.

Cao, Y., Liang, Y., Tanaka, K., Nguyen, C.T., Jedrzejczak, R.P., Joachimiak, A. and Stacey, G. (2014) The kinase LYK5 is a major chitin receptor in Arabidopsis and forms a chitin-induced complex with related kinase CERK1. Elife, 3, 1-19.

Capoen, W., Sun, J., Wysham, D., et al. (2011) Nuclear membranes control symbiotic calcium signaling of legumes. Proc. Natl. Acad. Sci. U. S. A., 108, 14348-14353.

Cappellazzo, G., Lanfranco, L., Fitz, M., Wipf, D. and Bonfante, P. (2008) Characterization of an amino acid permease from the endomycorrhizal fungus Glomus mosseae. Plant Physiol., 147, 429437.

Caris, C., Hördt, W., Hawkins, H.-J., Römheld, V. and George, E. (1998) Studies of iron transport by arbuscular mycorrhizal hyphae from soil to peanut and sorghum plants. Mycorrhiza, 8, 35-39.

Cartwright, G.M. and Scott, B. (2013) Redox regulation of an AP-1-like transcription factor, YapA, in the fungal symbiont Epichloe festucae. Eukaryot. Cell, 12, 1335-1348.

Chabaud, M., Harrison, M., Carvalho-niebel, F. De, Bécard, G., Barker, D.G. and Cedex, C.T. (2006) Inoculation and growth with mycorrhizal fungi. Medicago truncatula Handb., 1-15.

Chanclud, E. and Morel, J.-B. (2016) Plant hormones: a fungal point of view. Mol. Plant Pathol., 17, 1289-1297.

Charpentier, M., Sun, J., Martins, T. V., et al. (2016) Nuclearlocalized cyclic nucleotide-gated channels mediate symbiotic calcium oscillations. Science (80-. )., 352, 1102-1105.

Chaudhary, R., Atamian, H.S., Shen, Z., Briggs, S.P. and Kaloshian, I. (2014) GroEL from the endosymbiont Buchnera aphidicola betrays the aphid by triggering plant defense. Proc. Natl. Acad. Sci. U. S. A., 111, 8919-8924.

Chen, E.C.H., Morin, E., Beaudet, D., et al. (2018) High intraspecific genome diversity in the model arbuscular mycorrhizal symbiont Rhizophagus irregularis. New Phytol., 220, 1161-1171.

Chen, L.-Q. (2014) SWEET sugar transporters for phloem transport and pathogen nutrition. New Phytol., 201, 1150-1155.

Chen, L.-Q., Cheung, L.S., Feng, L., Tanner, W. and Frommer, W.B. (2015) Transport of sugars. Annu. Rev. Biochem., 84, 865-894.

Chen, L.Q., Hou, B.H., Lalonde, S., et al. (2010) Sugar transporters for intercellular exchange and nutrition of pathogens. Nature, 468, 527-532.

Chen, L.Q., Qu, X.Q., Hou, B.H., Sosso, D., Osorio, S., Fernie, A.R. and Frommer, W.B. (2012) Sucrose efflux mediated by SWEET proteins as a key step for phloem transport. Science (80-. )., 335, 207-211.

Chen, X.-L., Shi, T., Yang, J., et al. (2014) N-glycosylation of effector proteins by an a-1,3-mannosyltransferase is required for the rice 
blast fungus to evade host innate immunity. Plant Cell, 26, 13601376.

Chong, J., Piron, M.-C., Meyer, S., Merdinoglu, D., Bertsch, C. and Mestre, P. (2014) The SWEET family of sugar transporters in grapevine: VVSWEET4 is involved in the interaction with Botrytis cinerea. J. Exp. Bot., 65, 6589-6601.

Chorianopoulou, S.N., Saridis, Y.I., Dimou, M., Katinakis, P. and Bouranis, D.L. (2015) Arbuscular mycorrhizal symbiosis alters the expression patterns of three key iron homeostasis genes, ZmNAS1, ZmNAS3, and ZmYS1, in S deprived maize plants. Front. Plant Sci., 6, 257.

Conesa, A., Götz, S., García-Gómez, J.M., Terol, J., Talón, M. and Robles, M. (2005) Blast2GO: a universal tool for annotation, visualization and analysis in functional genomics research. Bioinformatics, 21, 3674-3676.

Cooper, B., Campbell, K.B., Beard, H.S., Garrett, W.M. and Islam, N. (2016) Putative rust fungal effector proteins in infected bean and soybean leaves. Phytopathology, 106, 491-499.

Cord-Landwehr, S., Melcher, R.L.J., Kolkenbrock, S. and Moerschbacher, B.M. (2016) A chitin deacetylase from the endophytic fungus Pestalotiopsis sp. efficiently inactivates the elicitor activity of chitin oligomers in rice cells. Sci. Rep., 6, 38018.

Corradi, N., Ruffner, B., Croll, D., Colard, A., Horák, A. and Sanders, I.R. (2009) High-level molecular diversity of copper-zinc superoxide dismutase genes among and within species of arbuscular Mycorrhizal fungi. Appl. Environ. Microbiol., 75, 1970-1978.

Crossley, D., Naraharisetty, V. and Shearer Glenmore, J. (2016) The Mould-specific M46 gene is not essential for yeast-mould dimorphism in the pathogenic fungus Histoplasma capsulatum. Med. Mycol., 54, 876-884.

Crossley, D.L. (2013) Investigating the function of the Mold Specific Gene M46, in the pathogenic dimorphic fungus Histoplasma capsulatum. PhD thesis, University of Southern Mississippi.

Cunningham, F.J., Goh, N.S., Demirer, G.S., Matos, J.L. and Landry, M.P. (2018) Nanoparticle-mediated delivery towards advancing plant genetic engineering. Trends Biotechnol., 36, 882-897.

Czaja, L.F., Hogekamp, C., Lamm, P., et al. (2012) Transcriptional responses toward diffusible signals from symbiotic microbes reveal MtNFP- and MtDMI3-dependent reprogramming of host gene expression by arbuscular mycorrhizal fungal lipochitooligosaccharides. Plant Physiol., 159, 1671-1685.

Dalal, R.C. (1977) Soil organic phosphorus. Adv. Agron., 29, 83-117.

Damme, M. van, Bozkurt, T.O., Cakir, C., Schornack, S., Sklenar, J., Jones, A.M.E. and Kamoun, S. (2012) The Irish potato famine Pathogen Phytophthora infestans translocates the CRN8 kinase into host plant cells. PLoS Pathog., 8, e1002875.

Day, D.A., Poole, P.S., Tyerman, S.D. and Rosendahl, L. (2001) 
Ammonia and amino acid transport across symbiotic membranes in nitrogen-fixing legume nodules. Cell. Mol. Life Sci., 58, 61-71.

Demchenko, K., Winzer, T., Stougaard, J., Parniske, M. and Pawlowski, K. (2004) Distinct roles of Lotus japonicus SYMRK and SYM15 in root colonization and arbuscule formation. New Phytol., 163, 381-392.

Dénarié, J., Debellé, F. and Promé, J.-C. (1996) Rhizobium lipochitooligosaccharide nodulation factors: signaling molecules mediating recognition and morphogenesis. Annu. Rev. Biochem., 65, 503-535.

Denyer, T., Ma, X., Klesen, S., Scacchi, E., Nieselt, K. and Timmermans, M.C.P. (2019) Spatiotemporal developmental trajectories in the Arabidopsis root revealed using high-throughput single-cell RNA sequencing. Dev. Cell, 48, 840-852.

Derevnina, L., Dagdas, Y.F., la Concepcion, J.C. De, et al. (2016) Nine things to know about elicitins. New Phytol., 212, 888-895.

Dickson, S. (2004) The Arum-Paris continuum of mycorrhizal symbioses. New Phytol., 163, 187-200.

Doehlemann, G., Linde, K. van der, Aßmann, D., Schwammbach, D., Hof, A., Mohanty, A., Jackson, D. and Kahmann, R. (2009) Pep1, a secreted effector protein of Ustilago maydis, is required for successful invasion of plant cells B. J. Howlett, ed. PLoS Pathog., 5, e1000290.

Doehlemann, G., Requena, N., Schaefer, P., Brunner, F., O'Connell, R. and Parker, J.E. (2014) Reprogramming of plant cells by filamentous plant-colonizing microbes. New Phytol., 204, 803-814.

Dou, D., Kale, S.D., Wang, X., Jiang, R.H.Y., Bruce, N. a, Arredondo, F.D., Zhang, X. and Tyler, B.M. (2008) RXLR-mediated entry of Phytophthora sojae effector Avr1b into soybean cells does not require pathogen-encoded machinery. Plant Cell, 20, 1930-1947.

Duplessis, S., Cuomo, C.A., Lin, Y.-C.Y., et al. (2011) Obligate biotrophy features unraveled by the genomic analysis of rust fungi. Proc. Natl. Acad. Sci. U. S. A., 108, 9166-9171.

Eichhorn, H., Lessing, F., Winterberg, B., Schirawski, J., Kämper, J., Müller, P. and Kahmann, R. (2006) A ferroxidation/permeation iron uptake system is required for virulence in Ustilago maydis. Plant Cell, 18, 3332-3345.

Endre, G., Kereszt, A., Kevei, Z., Mihacea, S., Kaló, P. and Kiss, G.B. (2002) A receptor kinase gene regulating symbiotic nodule development. Nature, 417, 962-966.

Engler, C., Youles, M., Gruetzner, R., Ehnert, T.-M., Werner, S., Jones, J.D.G., Patron, N.J. and Marillonnet, S. (2014) A golden gate modular cloning toolbox for plants. ACS Synth. Biol., 3, 839843.

Eom, J.-S., Chen, L.-Q., Sosso, D., Julius, B.T., Lin, I., Qu, X.-Q., Braun, D.M. and Frommer, W.B. (2015) SWEETs, transporters for intracellular and intercellular sugar translocation. Curr. Opin. Plant 
Biol., 25, 53-62.

Ezawa, T. and Saito, K. (2018) How do arbuscular mycorrhizal fungi handle phosphate? New insight into fine-tuning of phosphate metabolism. New Phytol., 220, 1116-1121.

Fellbaum, C.R., Gachomo, E.W., Beesetty, Y., Choudhari, S., Strahan, G.D. and Pfeffer, P.E. (2012) Carbon availability triggers fungal nitrogen uptake and transport in arbuscular mycorrhizal symbiosis. Proc. Natl. Acad. Sci. U. S. A., 109, 1-5.

Fesel, P.H. and Zuccaro, A. (2016) $\beta$-glucan: Crucial component of the fungal cell wall and elusive MAMP in plants. Fungal Genet. Biol., 90, $53-60$.

Floss, D.S., Gomez, S.K., Park, H.-J., MacLean, A.M., Müller, L.M., Bhattarai, K.K., Lévesque-Tremblay, V., Maldonado-Mendoza, I.E. and Harrison, M.J. (2017) A transcriptional program for arbuscule degeneration during AM symbiosis is regulated by MYB1. Curr. Biol., 27, 1206-1212.

Floss, D.S., Levy, J.G., Lévesque-Tremblay, V., Pumplin, N. and Harrison, M.J. (2013) DELLA proteins regulate arbuscule formation in arbuscular mycorrhizal symbiosis. Proc. Natl. Acad. Sci. U. S. A., 110, E5025-E5034.

García-Garrido, J.M. and Ocampo, J.A. (2002) Regulation of the plant defence response in arbuscular mycorrhizal symbiosis. J. Exp. Bot., 53, 1377-86.

Garcia, K., Chasman, D., Roy, S. and Ané, J.-M. (2017) Physiological responses and gene co-expression network of mycorrhizal roots under $\mathrm{K}^{+}$deprivation. Plant Physiol., 173, 1811-1823.

Gaude, N., Bortfeld, S., Duensing, N., Lohse, M. and Krajinski, F. (2012) Arbuscule-containing and non-colonized cortical cells of mycorrhizal roots undergo extensive and specific reprogramming during arbuscular mycorrhizal development. Plant J., 69, 510-528.

Gaude, N., Schulze, W.X., Franken, P. and Krajinski, F. (2012) Cell type-specific protein and transcription profiles implicate periarbuscular membrane synthesis as an important carbon sink in the mycorrhizal symbiosis. Plant Signal. Behav., 7, 461-464.

Genre, A., Chabaud, M., Balzergue, C., et al. (2013) Short-chain chitin oligomers from arbuscular mycorrhizal fungi trigger nuclear $\mathrm{Ca}^{2+}$ spiking in Medicago truncatula roots and their production is enhanced by strigolactone. New Phytol., 198, 190-202.

Genre, A., Chabaud, M., Faccio, A., Barker, D.G. and Bonfante, P. (2008) Prepenetration apparatus assembly precedes and predicts the colonization patterns of arbuscular mycorrhizal fungi within the root cortex of both Medicago truncatula and Daucus carota. Plant Cell, 20, 1407-20.

Genre, A., Chabaud, M., Timmers, T., Bonfante, P. and Barker, D.G. (2005) Arbuscular mycorrhizal fungi elicit a novel intracellular apparatus in Medicago truncatula root epidermal cells before infection. Plant Cell, 17, 3489-3499. 
Gibelin-Viala, C., Amblard, E., Puech-Pages, V., et al. (2019) The Medicago truncatula LysM receptor-ike kinase LYK9 plays a dual role in immunity and the arbuscular mycorrhizal symbiosis. New Phytol., 223, 1516-1529.

Gietz, R.D. and Schiestl, R.H. (2007) High-efficiency yeast transformation using the LiAc/SS carrier DNA/PEG method. Nat. Protoc., 2, 31-34.

Gimenez-Ibanez, S., Boter, M., Fernández-Barbero, G., Chini, A., Rathjen, J.P. and Solano, R. (2014) The bacterial effector HopX1 targets JAZ transcriptional repressors to activate jasmonate signaling and promote infection in Arabidopsis. PLoS Biol., 12, e1001792.

Giraldo, M.C., Dagdas, Y.F., Gupta, Y.K., et al. (2013) Two distinct secretion systems facilitate tissue invasion by the rice blast fungus Magnaporthe oryzae. Nat. Commun., 4, 1996.

Giraldo, M.C. and Valent, B. (2013) Filamentous plant pathogen effectors in action. Nat. Rev. Microbiol., 11, 800-814.

Gosling, P., Jones, J. and Bending, G.D. (2016) Evidence for functional redundancy in arbuscular mycorrhizal fungi and implications for agroecosystem management. Mycorrhiza, 26, 77-83.

Gough, C. and Cullimore, J. (2011) Lipo-chitooligosaccharide signaling in endosymbiotic plant-microbe interactions. Mol. plant-microbe Interact., 24, 867-878.

Govindarajulu, M., Pfeffer, P.E., Jin, H., Abubaker, J., Douds, D.D., Allen, J.W., Bücking, H., Lammers, P.J. and Shachar-Hill, Y. (2005) Nitrogen transfer in the arbuscular mycorrhizal symbiosis. Nature, 435, 819-823.

Groth, M., Takeda, N., Perry, J., et al. (2010) NENA, a Lotus japonicus homolog of Sec13, is required for rhizodermal infection by arbuscular mycorrhiza fungi and rhizobia but dispensable for cortical endosymbiotic development. Plant Cell, 22, 2509-2526.

Gueddari, N.E. El, Rauchhaus, U., Moerschbacher, B.M. and Deising, H.B. (2002) Developmentally regulated conversion of surface-exposed chitin to chitosan in cell walls of plant pathogenic fungi. New Phytol., 156, 103-112.

Guether, M, Balestrini, R., Hannah, M., He, J., Udvardi, M.K. and Bonfante, P. (2009a) Genome wide reprogramming of regulatory networks, transport, cell wall and membrane biogenesis during arbuscular mycorrhizal symbiosis in Lotus japonicus. New Phytol., 182, 200-212.

Guether, M, Neuhäuser, B., Balestrini, R., Dynowski, M., Ludewig, U. and Bonfante, P. (2009b) A mycorrhizal-specific ammonium transporter from Lotus japonicus acquires nitrogen released by arbuscular mycorrhizal fungi. Plant Physiol., 150, 73-83.

Guo, M., Chen, Y., Du, Y., et al. (2011) The bZIP transcription factor MoAP1 mediates the oxidative stress response and is critical for pathogenicity of the rice blast fungus Magnaporthe oryzae. PLOS Pathog., 7, e1001302. 
Gutjahr, C., Gobbato, E., Choi, J., et al. (2015) Rice perception of symbiotic arbuscular mycorrhizal fungi requires the karrikin receptor complex. Science (80-. )., 350, 1521-1524.

Gutjahr, C. and Parniske, M. (2013) Cell and developmental biology of arbuscular mycorrhiza symbiosis. Annu. Rev. Cell Dev. Biol., 29, 593-617.

Guyon, K., Balagué, C., Roby, D. and Raffaele, S. (2014) Secretome analysis reveals effector candidates associated with broad host range necrotrophy in the fungal plant pathogen Sclerotinia sclerotiorum. BMC Genomics, 15, 336.

Handa, Y., Nishide, H., Takeda, N., Suzuki, Y., Kawaguchi, M. and Saito, K. (2015) RNA-seq transcriptional profiling of an arbuscular mycorrhiza provides insights into regulated and coordinated gene expression in Lotus japonicus and Rhizophagus irregularis. Plant Cell Physiol., 56, 1490-1511.

Harrison, M.J. and Buuren, M.L. van (1995) A phosphate transporter from the mycorrhizal fungus Glomus versiforme. Nature, 378, 626629.

Harrison, M.J., Dewbre, G.R. and Liu, J. (2002) A phosphate transporter from Medicago truncatula involved in the acquisition of phosphate released by arbuscular mycorrhizal fungi. Plant Cell, 14, 2413-2429.

Häweker, H., Rips, S., Koiwa, H., Salomon, S., Saijo, Y., Chinchilla, D., Robatzek, S. and Schaewen, A. von (2010) Pattern recognition receptors require $\mathrm{N}$-glycosylation to mediate plant immunity. J. Biol. Chem., 285, 4629-4636.

Hawkins, H.-J., Johansen, A. and George, E. (2000) Uptake and transport of organic and inorganic nitrogen by arbuscular mycorrhizal fungi. Plant Soil, 226, 275-285.

Heijden, M.G.A. van der, Martin, F.M., Selosse, M.-A. and Sanders, I.R. (2015) Mycorrhizal ecology and evolution: the past, the present, and the future. New Phytol., 205, 1406-1423.

Helber, N. and Requena, N. (2008) Expression of the fluorescence markers DsRed and GFP fused to a nuclear localization signal in the arbuscular mycorrhizal fungus Glomus intraradices. New Phytol., 177, 537-548.

Helber, N., Wippel, K., Sauer, N., Schaarschmidt, S., Hause, B. and Requena, N. (2011) A versatile monosaccharide transporter that operates in the arbuscular mycorrhizal fungus Glomus $s p$ is crucial for the symbiotic relationship with plants. Plant Cell, 23, 3812-3823.

Helgason, T., Daniell, T.J., Husband, R., Fitter, A.H. and Young, J.P.W. (1998) Ploughing up the wood-wide web? Nature, 394, 431431.

Hemetsberger, C., Herrberger, C., Zechmann, B., Hillmer, M. and Doehlemann, G. (2012) The Ustilago maydis effector Pep1 suppresses plant immunity by inhibition of host peroxidase activity. PLoS Pathog., 8, e1002684. 
Hogekamp, C., Arndt, D., Pereira, P.A., Becker, J.D., Hohnjec, N. and Küster, H. (2011) Laser microdissection unravels cell-typespecific transcription in arbuscular mycorrhizal roots, including CAAT-box transcription factor gene expression correlating with fungal contact and spread. Plant Physiol., 157, 2023-43.

Hogekamp, C. and Küster, H. (2013) A roadmap of cell-type specific gene expression during sequential stages of the arbuscular mycorrhiza symbiosis. BMC Genomics, 14, 306.

Horváth, B., Yeun, L.H., Domonkos, Á., et al. (2011) Medicago truncatula IPD3 is a member of the common symbiotic signaling pathway required for rhizobial and mycorrhizal symbioses. Mol. Plant-Microbe Interact., 24, 1345-1358.

Huang, J., Gu, L., Zhang, Y., et al. (2017) An oomycete plant pathogen reprograms host pre-mRNA splicing to subvert immunity. Nat. Commun., 8, 2051.

Huisman, R., Bouwmeester, K., Brattinga, M., Govers, F., Bisseling, T. and Limpens, E. (2015) Haustorium formation in Medicago truncatula roots infected by Phytophthora palmivora does not involve the common endosymbiotic program shared by arbuscular mycorrhizal fungi and rhizobia. Mol. Plant-Microbe Interact., 28, 1271-1280.

Huisman, R., Hontelez, J., Mysore, K.S., Wen, J., Bisseling, T. and Limpens, E. (2016) A symbiosis-dedicated SYNTAXIN OF PLANTS 13 II isoform controls the formation of a stable host-microbe interface in symbiosis. New Phytol., 211, 1338-1351.

Inohara, N. and Nuñez, G. (2002) ML -- a conserved domain involved in innate immunity and lipid metabolism. Trends Biochem. Sci., 27, 219-221.

Ivanov, S., Austin, J., Berg, R.H. and Harrison, M.J. (2019) Extensive membrane systems at the host-arbuscular mycorrhizal fungus interface. Nat. Plants, 5, 194-203.

Ivanov, S., Fedorova, E.E., Limpens, E., Mita, S. De, Genre, A., Bonfante, P., Bisseling, T. and Mita, S. De (2012) Rhizobiumlegume symbiosis shares an exocytotic pathway required for arbuscule formation. Proc. Natl. Acad. Sci. U. S. A., 109, 83168321.

Ivanov, S. and Harrison, M.J. (2014) A set of fluorescent protein-based markers expressed from constitutive and arbuscular mycorrhizainducible promoters to label organelles, membranes and cytoskeletal elements in Medicago truncatula. Plant J., 80, 1151-1163.

Jakobsen, I. and Rosendahl, L. (1990) Carbon flow into soil and external hyphae from roots of mycorrhizal cucumber plants. New Phytol., 115, 77-83.

Jany, J. and Pawlowska, T.E. (2010) Multinucleate spores contribute to evolutionary longevity of asexual glomeromycota. Am. Nat., 175, 424-435.

Javot, H., Penmetsa, R.V., Terzaghi, N., Cook, D.R. and Harrison, 
M.J. (2007) A Medicago truncatula phosphate transporter indispensable for the arbuscular mycorrhizal symbiosis. Proc. Natl. Acad. Sci. U. S. A., 104, 1720-1725.

Jiang, Y., Wang, W., Xie, Q., et al. (2017) Plants transfer lipids to sustain colonization by mutualistic mycorrhizal and parasitic fungi. Science (80-. )., 356, 1172-1175.

Jiang, Y., Xie, Q., Wang, W., Yang, J., Zhang, X., Yu, N., Zhou, Y. and Wang, E. (2018) Medicago AP2-domain transcription factor WRI5a is a master regulator of lipid biosynthesis and transfer during mycorrhizal symbiosis. Mol. Plant, 11, 1344-1359.

Jin, Y., Chen, Z., Yang, J., Mysore, K.S., Wen, J., Huang, J., Yu, N. and Wang, E. (2018) IPD3 and IPD3L function redundantly in rhizobial and mycorrhizal symbioses. Front. Plant Sci., 9, 267.

Jin, Y., Liu, H., Luo, D., et al. (2016) DELLA proteins are common components of symbiotic rhizobial and mycorrhizal signalling pathways. Nat. Commun., 7, 12433.

Johnson, L. (2008) Iron and siderophores in fungal-host interactions. Mycol. Res., 112, 170-183.

Johnson, L.J., Koulman, A., Christensen, M., Lane, G.A., Fraser, K., Forester, N., Johnson, R.D., Bryan, G.T. and Rasmussen, S. (2013) An extracellular siderophore is required to maintain the mutualistic interaction of Epichloë festucae with Lolium perenne. PLoS Pathog., 9, e1003332.

Jonge, R. de, Esse, H.P. van, Kombrink, A., et al. (2010) Conserved fungal LysM effector Ecp6 prevents chitin-triggered immunity in plants. Science (80-. )., 329, 953-955.

Kafle, A., Garcia, K., Wang, X., Pfeffer, P.E., Strahan, G.D. and Bücking, H. (2019) Nutrient demand and fungal access to resources control the carbon allocation to the symbiotic partners in tripartite interactions of Medicago truncatula. Plant. Cell Environ., 42, 270284.

Kale, S.D., Gu, B., Capelluto, D.G.S., et al. (2010) External lipid PI3P mediates entry of eukaryotic pathogen effectors into plant and animal host cells. Cell, 142, 284-295.

Kamel, L., Tang, N., Malbreil, M., San Clemente, H., Marquer, M. Le, Roux, C. and Frei Dit Frey, N. (2017) The comparison of expressed candidate secreted proteins from two arbuscular mycorrhizal fungi unravels common and specific molecular tools to invade different host plants. Front. Plant Sci., 8, 124.

Kameoka, H., Maeda, T., Okuma, N. and Kawaguchi, M. (2019) Structure-specific regulation of nutrient transport and metabolism in arbuscular mycorrhizal fungi. Plant Cell Physiol., 0, 1-10.

Kameoka, H., Tsutsui, I., Saito, K., Kikuchi, Y., Handa, Y., Ezawa, T., Hayashi, H., Kawaguchi, M. and Akiyama, K. (2019) Stimulation of asymbiotic sporulation in arbuscular mycorrhizal fungi by fatty acids. Nat. Microbiol., 4, 1654-1660.

Kanamori, N., Madsen, L.H., Radutoiu, S., et al. (2006) A nucleoporin 
is required for induction of $\mathrm{Ca}^{2+}$ spiking in legume nodule development and essential for rhizobial and fungal symbiosis. Proc. Natl. Acad. Sci. U. S. A., 103, 359-364.

Kanno, Y., Oikawa, T., Chiba, Y., et al. (2016) AtSWEET13 and AtSWEET14 regulate gibberellin-mediated physiological processes. Nat. Commun., 7, 1-11.

Keymer, A. and Gutjahr, C. (2018) Cross-kingdom lipid transfer in arbuscular mycorrhiza symbiosis and beyond. Curr. Opin. Plant Biol., 44, 137-144.

Keymer, A., Pimprikar, P., Wewer, V., et al. (2017) Lipid transfer from plants to arbuscular mycorrhiza fungi. Elife, 6, 1-33.

Khang, C.H., Berruyer, R., Giraldo, M.C., Kankanala, P., Park, S.Y., Czymmek, K., Kang, S. and Valent, B. (2010) Translocation of Magnaporthe oryzae effectors into rice cells and their subsequent cell-to-cell movement. Plant Cell, 22, 1388-403.

Kiers, E.T., Duhamel, M., Beesetty, Y., et al. (2011) Reciprocal rewards stabilize cooperation in the mycorrhizal symbiosis. Science ( $80-$. )., 333, 880-882.

Kikuchi, Y., Hijikata, N., Ohtomo, R., Handa, Y., Kawaguchi, M., Saito, K., Masuta, C. and Ezawa, T. (2016) Aquaporin-mediated long-distance polyphosphate translocation directed towards the host in arbuscular mycorrhizal symbiosis: application of virus-induced gene silencing. New Phytol., 211, 1202-1208.

Kim, J.E., Kim, S.J., Lee, B.H., Park, R.W., Kim, K.S. and Kim, I.S. (2000) Identification of motifs for cell adhesion within the repeated domains of transforming growth factor-beta-induced gene, betaig-h3. J. Biol. Chem., 275, 30907-30915.

Kim, K.-T., Jeon, J., Choi, J., Cheong, K., Song, H., Choi, G., Kang, S. and Lee, Y.-H. (2016) Kingdom-wide analysis of fungal small secreted proteins (SSPs) reveals their potential role in host association. Front. Plant Sci., 7, 1-13.

Kistner, C., Winzer, T., Pitzschke, A., et al. (2005) Seven Lotus japonicus genes required for transcriptional reprogramming of the root during fungal and bacterial symbiosis. Plant Cell, 17, 22172229.

Kloppholz, S., Kuhn, H. and Requena, N. (2011) A secreted fungal effector of Glomus intraradices promotes symbiotic biotrophy. Curr. Biol., 21, 1204-1209.

Kobae, Y. and Hata, S. (2010) Dynamics of periarbuscular membranes visualized with a fluorescent phosphate transporter in arbuscular mycorrhizal roots of rice. Plant Cell Physiol., 51, 341-353.

Kobae, Y., Tamura, Y., Takai, S., Banba, M. and Hata, S. (2010) Localized expression of arbuscular mycorrhiza-inducible ammonium transporters in soybean. Plant Cell Physiol., 51, 1411-1415.

Koch, A.M., Antunes, P.M., Maherali, H., Hart, M.M. and Klironomos, J.N. (2017) Evolutionary asymmetry in the arbuscular mycorrhizal symbiosis: conservatism in fungal morphology does not predict host 
plant growth. New Phytol., 214, 1330-1337.

Koegel, S., Ait Lahmidi, N., Arnould, C., et al. (2013) The family of ammonium transporters (AMT) in Sorghum bicolor: two AMT members are induced locally, but not systemically in roots colonized by arbuscular mycorrhizal fungi. New Phytol., 198, 853-865.

Kohler, A., Kuo, A., Nagy, L.G., et al. (2015) Convergent losses of decay mechanisms and rapid turnover of symbiosis genes in mycorrhizal mutualists. Nat. Genet., 47, 410-415.

Kombrink, A., Rovenich, H., Shi-Kunne, X., et al. (2017) Verticillium dahliae LysM effectors differentially contribute to virulence on plant hosts. Mol. Plant Pathol., 18, 596-608.

Kombrink, A. and Thomma, B.P.H.J. (2013) LysM effectors: secreted proteins supporting fungal life. PLoS Pathog., 9, e1003769.

Kong, L., Qiu, X., Kang, J., et al. (2017) A Phytophthora effector manipulates host histone acetylation and reprograms defense gene expression to promote infection. Curr. Biol., 27, 981-991.

Krajinski, F., Courty, P.-E., Sieh, D., et al. (2014) The $\mathrm{H}^{+}$-ATPase HA1 of Medicago truncatula is essential for phosphate transport and plant growth during arbuscular mycorrhizal symbiosis. Plant Cell, 26, 1808-1817.

Kretzschmar, T., Kohlen, W., Sasse, J., et al. (2012) A petunia ABC protein controls strigolactone-dependent symbiotic signalling and branching. Nature, 483, 341-344.

Kryvoruchko, I.S., Sinharoy, S., Torres-Jerez, I., et al. (2016) MtSWEET11, a nodule-specific sucrose transporter of Medicago truncatula. Plant Physiol., 171, 554-565.

Kucera, M., Isserlin, R., Arkhangorodsky, A. and Bader, G.D. (2016) AutoAnnotate: A Cytoscape app for summarizing networks with semantic annotations. F1000Research, 5, 1717.

Kuge, S. and Jones, N. (1994) YAP1 dependent activation of TRX2 is essential for the response of Saccharomyces cerevisiae to oxidative stress by hydroperoxides. EMBO J., 13, 655-664.

Lahrmann, U., Ding, Y., Banhara, A., Rath, M., Hajirezaei, M.R., Döhlemann, S., Wirén, N. von, Parniske, M. and Zuccaro, A. (2013) Host-related metabolic cues affect colonization strategies of a root endophyte. Proc. Natl. Acad. Sci. U. S. A., 110, 13965-13970.

Lanfranco, L., Novero, M. and Bonfante, P. (2005) The mycorrhizal fungus Gigaspora margarita possesses a CuZn superoxide dismutase that is up-regulated during symbiosis with legume hosts. Plant Physiol., 137, 1319-1330.

Lee, J., Park, S.-H. and Eom, A.-H. (2006) Molecular identification of arbuscular mycorrhizal fungal spores collected in Korea. Mycobiology, 34, 7-13.

Lee, W.-S., Rudd, J.J., Hammond-Kosack, K.E. and Kanyuka, K. (2014) Mycosphaerella graminicola LysM effector-mediated stealth pathogenesis subverts recognition through both CERK1 and CEBiP homologues in Wheat. Mol. Plant-Microbe Interact., 27, 236-243. 
Lerouge, P., Roche, P., Faucher, C., Maillet, F., Truchet, G., Promé, J.C. and Dénarié, J. (1990) Symbiotic host-specificity of Rhizobium meliloti is determined by a sulphated and acylated glucosamine oligosaccharide signal. Nature, 344, 781-784.

Lévy, J., Bres, C., Geurts, R., et al. (2004) A putative $\mathrm{Ca}^{2+}$ and calmodulin-dependent protein kinase required for bacterial and fungal symbioses. Science (80-. )., 303, 1361-1364.

Liang, Y., Cao, Y., Tanaka, K., Thibivilliers, S., Wan, J., Choi, J., Kang, C. ho, Qiu, J. and Stacey, G. (2013) Nonlegumes respond to rhizobial Nod factors by suppressing the innate immune response. Science, 341, 1384-1387.

Limpens, E., Franken, C., Smit, P., Willemse, J., Bisseling, T. and Geurts, R. (2003) LysM domain receptor kinases regulating rhizobial Nod factor-induced infection. Science (80-. )., 302, 630-633.

Limpens, E., Ramos, J., Franken, C., Raz, V., Compaan, B., Franssen, H., Bisseling, T. and Geurts, R. (2004) RNA interference in Agrobacterium rhizogenes-transformed roots of Arabidopsis and Medicago truncatula. J. Exp. Bot., 55, 983-992.

Limpens, E., Zeijl, A. vanand Geurts, R. (2015) Lipochitooligosaccharides modulate plant host immunity to enable endosymbioses. Annu. Rev. Phytopathol., 53, 311-334.

Lin, I.W., Sosso, D., Chen, L.-Q., et al. (2014b) Nectar secretion requires sucrose phosphate synthases and the sugar transporter SWEET9. Nature, 508, 546-549.

Lin, K., Limpens, E., Zhang, Z., et al. (2014a) Single nucleus genome sequencing reveals high similarity among nuclei of an endomycorrhizal fungus. PLoS Genet., 10, e1004078.

Lindsay, P.L., Williams, B.N., MacLean, A. and Harrison, M.J. (2019) A phosphate-Dependent requirement for transcription factors IPD3 and IPD3L during arbuscular mycorrhizal symbiosis in Medicago truncatula. Mol. Plant-Microbe Interact., 32, 1277-1290.

Liu, Jianjian, Liu, Junli, Liu, Jinhui, Cui, M., Huang, Y., Tian, Y., Chen, A. and Xu, G. (2019) The potassium transporter SIHAK10 is involved in mycorrhizal potassium uptake. Plant Physiol., 180, 465-479.

Liu, T., Chen, G., Min, H. and Lin, F. (2009) MoFLP1, encoding a novel fungal fasciclin-like protein, is involved in conidiation and pathogenicity in Magnaporthe oryzae. J. Zhejiang Univ. Sci. B, 10, 434-444.

Liu, T., Liu, Z., Song, C., et al. (2012) Chitin-induced dimerization activates a plant immune receptor. Science (80-. )., 336, 11601164.

Liu, T., Song, T., Zhang, X., et al. (2014) Unconventionally secreted effectors of two filamentous pathogens target plant salicylate biosynthesis. Nat. Commun., 5, 4686.

Liu, W., Kohlen, W., Lillo, A., et al. (2011) Strigolactone biosynthesis in Medicago truncatula and rice requires the symbiotic GRAS-type 
transcription factors NSP1 and NSP2. Plant Cell, 23, 3853-3865.

Lodwig, E.M., Hosie, A.H.F., Bourdès, A., Findlay, K., Allaway, D., Karunakaran, R., Downie, J.A. and Poole, P.S. (2003) Aminoacid cycling drives nitrogen fixation in the legume-Rhizobium symbiosis. Nature, 422, 722-726.

López-Pedrosa, A., González-Guerrero, M., Valderas, A., AzcónAguilar, C. and Ferrol, N. (2006) GintAMT1 encodes a functional high-affinity ammonium transporter that is expressed in the extraradical mycelium of Glomus intraradices. Fungal Genet. Biol., 43, 102-110.

López-Ráez, J.A., Verhage, A., Fernández, I., García, J.M., AzcónAguilar, C., Flors, V. and Pozo, M.J. (2010) Hormonal and transcriptional profiles highlight common and differential host responses to arbuscular mycorrhizal fungi and the regulation of the oxylipin pathway. J. Exp. Bot., 61, 2589-2601.

Love, M.I., Huber, W. and Anders, S. (2014) Moderated estimation of fold change and dispersion for RNA-seq data with DESeq2. Genome Biol., 15, 550 .

Luginbuehl, L.H., Menard, G.N., Kurup, S., Erp, H. Van, Radhakrishnan, G. V., Breakspear, A., Oldroyd, G.E.D. and Eastmond, P.J. (2017) Fatty acids in arbuscular mycorrhizal fungi are synthesized by the host plant. Science (80-. )., 356, 1175-1178.

LuginbuehI, L.H. and Oldroyd, G.E.D. (2017) Understanding the arbuscule at the heart of endomycorrhizal symbioses in plants. Curr. Biol., 27, R952-R963.

Macheleidt, J., Mattern, D.J., Fischer, J., Netzker, T., Weber, J., Schroeckh, V., Valiante, V. and Brakhage, A.A. (2016) Regulation and role of fungal secondary metabolites. Annu. Rev. Genet., 50, 371-392.

Madej, M.G. (2014) Function, structure, and evolution of the major facilitator superfamily: The LacY manifesto. Adv. Biol., 2014, 1-20.

Maeda, T., Kobayashi, Y., Kameoka, H., Okuma, N., Takeda, N., Yamaguchi, K., Bino, T., Shigenobu, S. and Kawaguchi, M. (2018) Evidence of non-tandemly repeated rDNAs and their intragenomic heterogeneity in Rhizophagus irregularis. Commun. Biol., 1, 87.

Maere, S., Heymans, K. and Kuiper, M. (2005) BiNGO: A Cytoscape plugin to assess overrepresentation of gene ontology categories in biological networks. Bioinformatics, 21, 3448-3449.

Maillet, F., Poinsot, V., André, O., et al. (2011) Fungal lipochitooligosaccharide symbiotic signals in arbuscular mycorrhiza. Nature, 469, 58-63.

Maldonado-Mendoza, I.E., Dewbre, G.R. and Harrison, M.J. (2001) A phosphate transporter gene from the extra-radical mycelium of an arbuscular mycorrhizal fungus Glomus intraradices is regulated in response to phosphate in the environment. Mol. plant-microbe Interact., 14, 1140-1148. 
Malonek, S., Bömke, C., Bornberg-Bauer, E., Rojas, M.C., Hedden, P., Hopkins, P. and Tudzynski, B. (2005) Distribution of gibberellin biosynthetic genes and gibberellin production in the Gibberella fujikuroi species complex. Phytochemistry, 66, 1296-1311.

Manck-Götzenberger, J. and Requena, N. (2016) Arbuscular mycorrhiza symbiosis induces a major transcriptional reprogramming of the potato SWEET sugar transporter family. Front. Plant Sci., 7, 487.

Marleau, J., Dalpé, Y., St-Arnaud, M. and Hijri, M. (2011) Spore development and nuclear inheritance in arbuscular mycorrhizal fungi. BMC Evol. Biol., 11, 51.

Marquer, M. Le, Bécard, G. and Frei dit Frey, N. (2019) Arbuscular mycorrhizal fungi possess a CLAVATA3/embryo surrounding regionrelated gene that positively regulates symbiosis. New Phytol., 222, 1030-1042.

Marshall, R., Kombrink, A., Motteram, J., Loza-Reyes, E., Lucas, J., Hammond-Kosack, K.E., Thomma, B.P.H.J. and Rudd, J.J. (2011) Analysis of two in planta expressed LysM effector homologs from the fungus Mycosphaerella graminicola reveals novel functional properties and varying contributions to virulence on wheat. Plant Physiol., 156, 756-769.

Mateus, I.D., Masclaux, F.G., Aletti, C., Rojas, E.C., Savary, R., Dupuis, C. and Sanders, I.R. (2019) Dual RNA-seq reveals large-scale non-conserved genotype $\times$ genotype-specific genetic reprograming and molecular crosstalk in the mycorrhizal symbiosis. ISME J., 13, 1226-1238.

McGONIGLE, T.P., MILLER, M.H., EVANS, D.G., FAIRCHILD, G.L. and SWAN, J.A. (1990) A new method which gives an objective measure of colonization of roots by vesicular-arbuscular mycorrhizal fungi. New Phytol., 115, 495-501.

Mentlak, T.A., Kombrink, A., Shinya, T., et al. (2012) Effectormediated suppression of chitin-triggered immunity by Magnaporthe oryzae is necessary for rice blast disease. Plant Cell, 24, 322-335.

Merico, D., Isserlin, R., Stueker, O., Emili, A. and Bader, G.D. (2010) Enrichment map: A network-based method for gene-set enrichment visualization and interpretation. PLoS One, 5, e13984.

Messinese, E., Mun, J.-H., Yeun, L.H., et al. (2007) A novel nuclear protein interacts with the symbiotic DMI3 calcium- and calmodulindependent protein kinase of Medicago truncatula. Mol. plant-microbe Interact., 20, 912-921.

Mikes, V., Milat, M.L., Ponchet, M., Panabières, F., Ricci, P. and Blein, J.P. (1998) Elicitins, proteinaceous elicitors of plant defense, are a new class of sterol carrier proteins. Biochem. Biophys. Res. Commun., 245, 133-139.

Miya, A., Albert, P., Shinya, T., et al. (2007) CERK1, a LysM receptor kinase, is essential for chitin elicitor signaling in Arabidopsis. Proc. Natl. Acad. Sci. U. S. A., 104, 19613-19618. 
Miyata, K., Kozaki, T., Kouzai, Y., et al. (2014) The bifunctional plant receptor, OsCERK1, regulates both chitin-triggered immunity and arbuscular mycorrhizal symbiosis in rice. Plant Cell Physiol., 55, 1864-1872.

Montibus, M., Pinson-Gadais, L., Richard-Forget, F., Barreau, C. and Ponts, N. (2015) Coupling of transcriptional response to oxidative stress and secondary metabolism regulation in filamentous fungi. Crit. Rev. Microbiol., 41, 295-308.

Moore, J.W., Herrera-Foessel, S., Lan, C., et al. (2015) A recently evolved hexose transporter variant confers resistance to multiple pathogens in wheat. Nat. Genet., 47, 1494-1498.

Mueller, O., Kahmann, R., Aguilar, G., Trejo-Aguilar, B., Wu, A. and Vries, R.P. de (2008) The secretome of the maize pathogen Ustilago maydis. Fungal Genet. Biol., 45, S63-S70.

Müller, O., Schreier, P.H. and Uhrig, J.F. (2008) Identification and characterization of secreted and pathogenesis-related proteins in Ustilago maydis. Mol. Genet. Genomics, 279, 27-39.

Murray, J.D., Muni, R.R.D., Torres-Jerez, I., et al. (2011) Vapyrin, a gene essential for intracellular progression of arbuscular mycorrhizal symbiosis, is also essential for infection by rhizobia in the nodule symbiosis of Medicago truncatula. Plant J., 65, 244-252.

Nadal, M., Sawers, R., Naseem, S., et al. (2017) An N-acetylglucosamine transporter required for arbuscular mycorrhizal symbioses in rice and maize. Nat. Plants, 3, 17073.

Nars, A., Rey, T., Lafitte, C., et al. (2013) An experimental system to study responses of Medicago truncatula roots to chitin oligomers of high degree of polymerization and other microbial elicitors. Plant Cell Rep., 32, 489-502.

Neupane, A., Feng, C., Feng, J., Kafle, A., Bücking, H. and Lee Marzano, S.-Y. (2018) Metatranscriptomic analysis and in silico approach identified mycoviruses in the arbuscular mycorrhizal fungus Rhizophagus spp. Viruses, 10, 707.

Nielsen, H. and Krogh, A. (1998) Prediction of signal peptides and signal anchors by a hidden Markov model. Intell. Syst. Mol. Biol., 6, 122-130.

Nobori, T., Velásquez, A.C., Wu, J., Kvitko, B.H., Kremer, J.M., Wang, Y., He, S.Y. and Tsuda, K. (2018) Transcriptome landscape of a bacterial pathogen under plant immunity. Proc. Natl. Acad. Sci. U. S. A., 115, E3055-E3064.

Oide, S., Moeder, W., Krasnoff, S., Gibson, D., Haas, H., Yoshioka, K. and Turgeon, B.G. (2006) NPS6, encoding a nonribosomal peptide synthetase involved in siderophore-mediated iron metabolism, is a conserved virulence determinant of plant pathogenic ascomycetes. Plant Cell, 18, 2836-2853.

Ökmen, B., Etalo, D.W., Joosten, M.H.A.J., Bouwmeester, H.J., Vos, R.C.H. de, Collemare, J. and Wit, P.J.G.M. de (2013) Detoxification of a-tomatine by Cladosporium fulvum is required for 
full virulence on tomato. New Phytol., 198, 1203-1214.

Oláh, B., Brière, C., Bécard, G., Dénarié, J. and Gough, C. (2005) Nod factors and a diffusible factor from arbuscular mycorrhizal fungi stimulate lateral root formation in Medicago truncatula via the DMI1/ DMI2 signalling pathway. Plant J., 44, 195-207.

Oldroyd, G.E.D. (2013) Speak, friend, and enter: signalling systems that promote beneficial symbiotic associations in plants. Nat. Rev. Microbiol., 11, 252-263.

Ovchinnikova, E., Journet, E.-P., Chabaud, M., et al. (2011) IPD3 controls the formation of nitrogen-fixing symbiosomes in pea and Medicago Spp. Mol. Plant. Microbe. Interact., 24, 1333-1344.

Pareja-Jaime, Y., Roncero, M.I.G. and Ruiz-Roldán, M.C. (2008) Tomatinase from Fusarium oxysporum f. sp. lycopersici is required for full virulence on tomato plants. Mol. Plant-Microbe Interact., 21, 728-736.

Park, H.-J., Floss, D.S., Levesque-Tremblay, V., Bravo, A. and Harrison, M.J. (2015) Hyphal branching during arbuscule development requires Reduced Arbuscular Mycorrhiza1. Plant Physiol., 169, 2774-2788.

Paulsrud, P. and Lindblad, P. (2002) Fasciclin domain proteins are present in nostoc symbionts of lichens. Appl. Environ. Microbiol., 68, 2036-2039.

Pedras, M.S.C. and Ahiahonu, P.W.K. (2005) Metabolism and detoxification of phytoalexins and analogs by phytopathogenic fungi. Phytochemistry, 66, 391-411.

Pérez-Tienda, J., Testillano, P.S., Balestrini, R., Fiorilli, V., AzcónAguilar, C. and Ferrol, N. (2011) GintAMT2, a new member of the ammonium transporter family in the arbuscular mycorrhizal fungus Glomus intraradices. Fungal Genet. Biol., 48, 1044-1055.

Petersen, T.N., Brunak, S., Heijne, G. von and Nielsen, H. (2011) SignalP 4.0: discriminating signal peptides from transmembrane regions. Nat. Methods, 8, 785-786.

Petre, B. and Kamoun, S. (2014) How do filamentous pathogens deliver effector proteins into plant cells? PLoS Biol., 12, e1001801.

Pfeffer, Douds Jr DD, Becard and Shachar-Hill (1999) Carbon uptake and the metabolism and transport of lipids in an arbuscular mycorrhiza. Plant Physiol., 120, 587-598.

Pimprikar, P., Carbonnel, S., Paries, M., et al. (2016) A CCaMKCYCLOPS-DELLA complex activates transcription of RAM1 to regulate arbuscule branching. Curr. Biol., 26, 987-998.

Pimprikar, P. and Gutjahr, C. (2018) Transcriptional regulation of arbuscular mycorrhiza development. Plant Cell Physiol., 59, 673690.

Plett, J.M., Daguerre, Y., Wittulsky, S., et al. (2014) Effector MiSSP7 of the mutualistic fungus Laccaria bicolor stabilizes the Populus JAZ6 protein and represses jasmonic acid (JA) responsive genes. Proc. Natl. Acad. Sci. U. S. A., 111, 8299-8304. 
Plett, J.M., Kemppainen, M., Kale, S.D., Kohler, A., Legué, V., Brun, A., Tyler, B.M., Pardo, A.G. and Martin, F. (2011) A secreted effector protein of Laccaria bicolor is required for symbiosis development. Curr. Biol., 21, 1197-1203.

Plett, J.M. and Martin, F. (2015) Reconsidering mutualistic plantfungal interactions through the lens of effector biology. Curr. Opin. Plant Biol., 26, 45-50.

Pozo, M.J., Azcón-Aguilar, C., Dumas-Gaudot, E. and Barea, J.M. (1998) Chitosanase and chitinase activities in tomato roots during interactions with arbuscular mycorrhizal fungi or Phytophthora parasitica. J. Exp. Bot., 49, 1729-1739.

Prasad Singh, P., Srivastava, D., Jaiswar, A. and Adholeya, A. (2019) Effector proteins of Rhizophagus proliferus: conserved protein domains may play a role in host-specific interaction with different plant species. Brazilian J. Microbiol., 50, 593-601.

Presti, L. Lo and Kahmann, R. (2017) How filamentous plant pathogen effectors are translocated to host cells. Curr. Opin. Plant Biol., 38, $19-24$.

Presti, L. Lo, Lanver, D., Schweizer, G., Tanaka, S., Liang, L., Tollot, M., Zuccaro, A., Reissmann, S. and Kahmann, R. (2015) Fungal effectors and plant susceptibility. Annu. Rev. Plant Biol., 66, 513-545.

Puga, M.I., Mateos, I., Charukesi, R., et al. (2014) SPX1 is a phosphatedependent inhibitor of PHOSPHATE STARVATION RESPONSE 1 in Arabidopsis. Proc. Natl. Acad. Sci. U. S. A., 111, 14947-14952.

Pumplin, N., Mondo, S.J., Topp, S., Starker, C.G., Gantt, J.S. and Harrison, M.J. (2010) Medicago truncatula Vapyrin is a novel protein required for arbuscular mycorrhizal symbiosis. Plant J., 61, 482-494.

Pumplin, N., Zhang, X., Noar, R.D. and Harrison, M.J. (2012) Polar localization of a symbiosis-specific phosphate transporter is mediated by a transient reorientation of secretion. Proc. Natl. Acad. Sci. U. S. A., 109, E665-E672.

Radutoiu, S., Madsen, L.H., Madsen, E.B., et al. (2003) Plant recognition of symbiotic bacteria requires two LysM receptor-like kinases. Nature, 425, 585-592.

Remy, W., Taylor, T.N., Hass, H. and Kerp, H. (1994) Four hundredmillion-year-old vesicular arbuscular mycorrhizae. Proc. Natl. Acad. Sci. U. S. A., 91, 11841-11843.

Ren, B., Wang, X., Duan, J. and Ma, J. (2019) Rhizobial tRNA-derived small RNAs are signal molecules regulating plant nodulation. Science (80-. )., 365, 919-922.

Rey, T., Andr, O., Nars, A., Dumas, B., Gough, C., Bottin, A. and Jacquet, C. (2018) Rapid report lipo-chitooligosaccharide signalling blocks a rapid pathogen-induced ROS burst without impeding immunity. New Phytol., 221, 743-749.

Rich, M.K., Nouri, E., Courty, P.-E. and Reinhardt, D. (2017) Diet of 
arbuscular mycorrhizal fungi: bread and butter? Trends Plant Sci., 22, 652-660.

Rich, M.K., Schorderet, M., Bapaume, L., Falquet, L., Morel, P., Vandenbussche, M. and Reinhardt, D. (2015) The Petunia GRAS transcription factor ATA/RAM1 regulates symbiotic gene expression and fungal morphogenesis in arbuscular mycorrhiza. Plant Physiol., 168, 788-797.

Ried, M.K., Antolín-Llovera, M. and Parniske, M. (2014) Spontaneous symbiotic reprogramming of plant roots triggered by receptor-like kinases. Elife, 3, e03891.

Riely, B.K., Lougnon, G., Ané, J.M. and Cook, D.R. (2007) The symbiotic ion channel homolog DMI1 is localized in the nuclear membrane of Medicago truncatula roots. Plant J., 49, 208-216.

Roche, Philippe, Debellé, F., Maillet, F., Lerouge, P., Faucher, C., Truchet, G., Dénarié, J. and Promé, J.-C. (1991) Molecular basis of symbiotic host specificity in rhizobium meliloti: nodH and nodPQ genes encode the sulfation of lipo-oligosaccharide signals. Cell, 67, 1131-1143.

Roche, P, Lerouge, P., Ponthus, C. and Promé, J.C. (1991) Structural determination of bacterial nodulation factors involved in the Rhizobium meliloti-alfalfa symbiosis. J. Biol. Chem., 266, 1093310940.

Ropars, J., Toro, K.S., Noel, J., et al. (2016) Evidence for the sexual origin of heterokaryosis in arbuscular mycorrhizal fungi. Nat. Microbiol., 1, 16033.

Roth, R., Hillmer, S., Funaya, C., Chiapello, M., Schumacher, K., Presti, L. Lo, Kahmann, R. and Paszkowski, U. (2019) Arbuscular cell invasion coincides with extracellular vesicles and membrane tubules. Nat. Plants, 5, 204-211.

Roth, R. and Paszkowski, U. (2017) Plant carbon nourishment of arbuscular mycorrhizal fungi. Curr. Opin. Plant Biol., 39, 50-56.

Rovenich, H., Zuccaro, A. and Thomma, B.P.H.J. (2016) Convergent evolution of filamentous microbes towards evasion of glycantriggered immunity. New Phytol., 212, 896-901.

Rutter, B.D. and Innes, R.W. (2018) Extracellular vesicles as key mediators of plant-microbe interactions. Curr. Opin. Plant Biol., 44, 16-22.

Ryu, K.H., Huang, L., Kang, H.M. and Schiefelbein, J. (2019) Single-Cell RNA sequencing resolves molecular relationships among individual plant cells. Plant Physiol., 179, 1444-1456.

Saito, K., Yoshikawa, M., Yano, K., et al. (2007) NUCLEOPORIN85 is required for calcium spiking, fungal and bacterial symbioses, and seed production in Lotus japonicus. Plant Cell, 19, 610-624.

Salvioli, A., Ghignone, S., Novero, M., Navazio, L., Venice, F., Bagnaresi, P. and Bonfante, P. (2016) Symbiosis with an endobacterium increases the fitness of a mycorrhizal fungus, raising its bioenergetic potential. ISME J., 10, 130-144. 
Sánchez-Vallet, A., Mesters, J.R. and Thomma, B.P.H.J.H.J. (2015) The battle for chitin recognition in plant-microbe interactions. FEMS Microbiol. Rev., 39, 171-183.

Sánchez-Vallet, A., Saleem-Batcha, R., Kombrink, A., Hansen, G., Valkenburg, D.J., Thomma, B.P.H.J. and Mesters, J.R. (2013) Fungal effector Ecp6 outcompetes host immune receptor for chitin binding through intrachain LysM dimerization. Elife, 2013, 1-16.

Sanders, I.R. (2010) 'Designer' mycorrhizas?: Using natural genetic variation in AM fungi to increase plant growth. ISME J., 4, 10811083.

Sanders, I.R. and Croll, D. (2010) Arbuscular mycorrhiza: the challenge to understand the genetics of the fungal partner. Annu. Rev. Genet., 44, 271-292.

Sato, T., Ezawa, T., Cheng, W. and Tawaraya, K. (2015) Release of acid phosphatase from extraradical hyphae of arbuscular mycorrhizal fungus Rhizophagus clarus. Soil Sci. Plant Nutr., 61, 269-274.

Sauer, N. and Stolz, J. (1994) SUC1 and SUC2: two sucrose transporters from Arabidopsis thaliana; expression and characterization in baker's yeast and identification of the histidine-tagged protein. Plant J., 6, 67-77.

Sawers, R.J.H., Svane, S.F., Quan, C., et al. (2017) Phosphorus acquisition efficiency in arbuscular mycorrhizal maize is correlated with the abundance of root-external hyphae and the accumulation of transcripts encoding PHT1 phosphate transporters. New Phytol., 214, 632-643.

Schaarschmidt, S., Kopka, J., Ludwig-Müller, J. and Hause, B. (2007) Regulation of arbuscular mycorrhization by apoplastic invertases: enhanced invertase activity in the leaf apoplast affects the symbiotic interaction. Plant J., 51, 390-405.

Schmitz, A.M., Pawlowska, T.E. and Harrison, M.J. (2019) A short LysM protein with high molecular diversity from an arbuscular mycorrhizal fungus, Rhizophagus irregularis. Mycoscience, 60, 6370.

Schornack, S., Damme, M. van, Bozkurt, T.O., Cano, L.M., Smoker, M., Thines, M., Gaulin, E., Kamoun, S. and Huitema, E. (2010) Ancient class of translocated oomycete effectors targets the host nucleus. Proc. Natl. Acad. Sci. U. S. A., 107, 17421-17426.

Schultze, M., Quiclet-Sire, B., Kondorosi, E., Virelizer, H., Glushka, J.N., Endre, G., Géro, S.D. and Kondorosi, A. (1992) Rhizobium meliloti produces a family of sulfated lipooligosaccharides exhibiting different degrees of plant host specificity. Proc. Natl. Acad. Sci. U. S. A., 89, 192-6.

Sędzielewska Toro, K. and Brachmann, A. (2016) The effector candidate repertoire of the arbuscular mycorrhizal fungus Rhizophagus clarus. BMC Genomics, 17, 101.

Seifert, G.J. (2018) Fascinating fasciclins: A surprisingly widespread family of proteins that mediate interactions between the cell exterior 
and the cell surface. Int. J. Mol. Sci., 19.

Selin, C., Kievit, T.R. de, Belmonte, M.F. and Fernando, W.G.D. (2016) Elucidating the role of effectors in plant-fungal interactions: progress and challenges. Front. Microbiol., 7, 1-21.

Shachar-Hill, Y., Pfeffer, P.E., Douds, D., Osman, S.F., Doner, L.W. and Ratcliffe, R.G. (1995) Partitioning of intermediary carbon metabolism in vesicular-arbuscular mycorrhizal leek. Plant Physiol., 108, 7-15.

Shannon, P., Markiel, A., Ozier, O., Baliga, N.S., Wang, J.T., Ramage, D., Amin, N., Schwikowski, B. and Ideker, T. (2003) Cytoscape: a software environment for integrated models of biomolecular interaction networks. Genome Res., 13, 2498-2504.

Shimizu, T., Nakano, T., Takamizawa, D., et al. (2010) Two LysM receptor molecules, CEBiP and OsCERK1, cooperatively regulate chitin elicitor signaling in rice. Plant J., 64, 204-214.

Sieberer, B.J., Chabaud, M., Fournier, J., Timmers, A.C.J. and Barker, D.G. (2012) A switch in $\mathrm{Ca}^{2+}$ spiking signature is concomitant with endosymbiotic microbe entry into cortical root cells of Medicago truncatula. Plant J., 69, 822-830.

Singh, S., Katzer, K., Lambert, J., Cerri, M. and Parniske, M. (2014) CYCLOPS, a DNA-binding transcriptional activator, orchestrates symbiotic root nodule development. Cell Host Microbe, 15, 139-152.

Smith, S.E. and Read, D. (2008) Mycorrhizal Symbiosis (Third Edition), London: Academic press.

Smith, S.E. and Smith, F.A. (2011) Roles of arbuscular mycorrhizas in plant nutrition and growth: new paradigms from cellular to ecosystem scales. Annu. Rev. Plant Biol., 62, 227-250.

Snelders, N.C., Kettles, G.J., Rudd, J.J. and Thomma, B.P.H.J. (2018) Plant pathogen effector proteins as manipulators of host microbiomes? Mol. Plant Pathol., 19, 257-259.

Solaiman, M.Z. and Saito, M. (1997) Use of sugars by intraradical hyphae of arbuscular mycorrhizal fungi revealed by radiorespirometry. New Phytol., 136, 533-538.

Song, T., Ma, Z., Shen, D., et al. (2015) An oomycete CRN effector reprograms expression of plant HSP genes by targeting their promoters. PLOS Pathog., 11, e1005348.

Spatafora, J.W., Chang, Y., Benny, G.L., et al. (2016) A phylum-level phylogenetic classification of zygomycete fungi based on genomescale data. Mycologia, 108, 1028-1046.

Sperschneider, J., Catanzariti, A.-M., DeBoer, K., Petre, B., Gardiner, D.M., Singh, K.B., Dodds, P.N. and Taylor, J.M. (2017) LOCALIZER: subcellular localization prediction of both plant and effector proteins in the plant cell. Sci. Rep., 7, 44598.

Srinivasan, M., Kumar, K., Kumutha, K. and Marimuthu, P. (2014) Establishing monoxenic culture of arbuscular mycorrhizal fungus Glomus intraradices through root organ culture. J. Appl. Nat. Sci., 6, 290-293. 
Staehelin, C., Schultze, M., Tokuyasu, K., Poinsot, V., Promé, J.C., Kondorosi, E. and Kondorosi, A. (2000) N-deacetylation of Sinorhizobium meliloti Nod factors increases their stability in the Medicago sativa rhizosphere and decreases their biological activity. Mol. plant-microbe Interact., 13, 72-79.

Stassen, J.H. and Ackerveken, G. Van den (2011) How do oomycete effectors interfere with plant life? Curr. Opin. Plant Biol., 14, 407414.

Stracke, S., Kistner, C., Yoshida, S., et al. (2002) A plant receptorlike kinase required for both bacterial and fungal symbiosis. Nature, 417, 959-962.

Stringlis, I.A., Yu, K., Feussner, K., et al. (2018) MYB72-dependent coumarin exudation shapes root microbiome assembly to promote plant health. Proc. Natl. Acad. Sci. U. S. A., 115, E5213-E5222.

Sugimura, Y. and Saito, K. (2017) Comparative transcriptome analysis between Solanum lycopersicum L. and Lotus japonicus L. during arbuscular mycorrhizal development. Soil Sci. Plant Nutr., 63, 127136.

Sugiura, Y., Akiyama, R., Tanaka, S., Yano, K., Kameoka, H., Kawaguchi, M., Akiyama, K. and Saito, K. (2019) Myristate as a carbon and energy source for the asymbiotic growth of the arbuscular mycorrhizal fungus Rhizophagus irregularis. bioRxiv, 731489.

Sun, X., Chen, W., Ivanov, S., MacLean, A.M., Wight, H., Ramaraj, T., Mudge, J., Harrison, M.J. and Fei, Z. (2019) Genome and evolution of the arbuscular mycorrhizal fungus Diversispora epigaea (formerly Glomus versiforme ) and its bacterial endosymbionts. New Phytol., 221, 1556-1573.

Tadege, M., Wen, J., He, J., et al. (2008) Large-scale insertional mutagenesis using the Tnt1 retrotransposon in the model legume Medicago truncatula. Plant J., 54, 335-347.

Takahara, H., Hacquard, S., Kombrink, A., et al. (2016) Colletotrichum higginsianum extracellular LysM proteins play dual roles in appressorial function and suppression of chitin-triggered plant immunity. New Phytol., 211, 1323-1337.

Takeda, N., Handa, Y., Tsuzuki, S., Kojima, M., Sakakibara, H. and Kawaguchi, M. (2015) Gibberellins interfere with symbiosis signaling and gene expression and alter colonization by arbuscular mycorrhizal fungi in Lotus japonicus. Plant Physiol., 167, 545-557.

Takeda, N., Maekawa, T. and Hayashi, M. (2012) Nuclear-localized and deregulated calcium- and calmodulin-dependent protein kinase activates rhizobial and mycorrhizal responses in Lotus japonicus. Plant Cell, 24, 810-822.

Taleski, M., Imin, N. and Djordjevic, M.A. (2018) CEP peptide hormones: key players in orchestrating nitrogen-demand signalling, root nodulation, and lateral root development. J. Exp. Bot., 69, 1829-1836.

Tamayo, E., Gómez-Gallego, T., Azcón-Aguilar, C. and Ferrol, N. 
(2014) Genome-wide analysis of copper, iron and zinc transporters in the arbuscular mycorrhizal fungus Rhizophagus irregularis. Front. Plant Sci., 5, 547.

Tamayo, E., Knight, S.A.B., Valderas, A., Dancis, A. and Ferrol, N. (2018) The arbuscular mycorrhizal fungus Rhizophagus irregularis uses a reductive iron assimilation pathway for high-affinity iron uptake. Environ. Microbiol., 20, 1857-1872.

Tamura, K., Stecher, G., Peterson, D., Filipski, A. and Kumar, S. (2013) MEGA6: Molecular Evolutionary Genetics Analysis version 6.0. Mol. Biol. Evol., 30, 2725-2729.

Tang, H., Krishnakumar, V., Bidwell, S., et al. (2014) An improved genome release (version Mt4.0) for the model legume Medicago truncatula. BMC Genomics, 15, 312.

Tang, N., San Clemente, H., Roy, S., Bécard, G., Zhao, B. and Roux, C. (2016) A Survey of the gene repertoire of Gigaspora rosea unravels conserved features among glomeromycota for obligate biotrophy. Front. Microbiol., 7, 1-16.

Tao, Y., Cheung, L.S., Li, S., Eom, J.-S., Chen, L.-Q., Xu, Y., Perry, K., Frommer, W.B. and Feng, L. (2015) Structure of a eukaryotic SWEET transporter in a homotrimeric complex. Nature, 527, 259263.

Tarazona, S., Furió-Tarí, P., Turrà, D., Pietro, A. Di, Nueda, M.J., Ferrer, A. and Conesa, A. (2015) Data quality aware analysis of differential expression in RNA-seq with NOISeq R/Bioc package. Nucleic Acids Res., 43, e140.

Tian, C., Kasiborski, B., Koul, R., Lammers, P.J., Bucking, H. and Shachar-Hill, Y. (2010) Regulation of the nitrogen transfer pathway in the arbuscular mycorrhizal symbiosis: gene characterization and the coordination of expression with nitrogen flux. Plant Physiol., 153, 1175-1187.

Tirichine, L., Imaizumi-Anraku, H., Yoshida, S., et al. (2006) Deregulation of a $\mathrm{Ca}^{2+} /$ calmodulin-dependent kinase leads to spontaneous nodule development. Nature, 441, 1153-1156.

Tisserant, E., Kohler, a, Dozolme-Seddas, P., et al. (2012) The transcriptome of the arbuscular mycorrhizal fungus Glomus intraradices (DAOM 197198) reveals functional tradeoffs in an obligate symbiont. New Phytol., 193, 755-769.

Tisserant, E., Malbreil, M., Kuo, A., et al. (2013) Genome of an arbuscular mycorrhizal fungus provides insight into the oldest plant symbiosis. Proc. Natl. Acad. Sci. U. S. A., 110, 20117-20122.

Toone, W.M., Morgan, B.A. and Jones, N. (2001) Redox control of AP-1-like factors in yeast and beyond. Oncogene, 20, 2336-2346.

Torrecillas, E., Alguacil, M.M., Roldan, A., Roldán, A. and Roldan, A. (2012) Host preferences of arbuscular mycorrhizal fungi colonizing annual herbaceous plant species in semiarid Mediterranean prairies. Appl. Environ. Microbiol., 78, 6180-6186.

Trouvelot, A., Kough, J.L. and Gianinazzi-Pearson, V. (1986) Mesure 
du taux de mycorhization VA d'un systeme radiculaire. Recherche de methodes d'estimation ayant une signification fonctionnelle. In Physiological and genetical aspects of mycorrhizae. Paris: INRA press, pp. 217-221.

Tsuzuki, S., Handa, Y., Takeda, N. and Kawaguchi, M. (2016) Strigolactone-induced Putative Secreted Protein 1 is required for the establishment of symbiosis by the arbuscular mycorrhizal fungus Rhizophagus irregularis. Mol. Plant-Microbe Interact., 29, 277-286.

Turina, M., Ghignone, S., Astolfi, N., Silvestri, A., Bonfante, P. and Lanfranco, L. (2018) The virome of the arbuscular mycorrhizal fungus Gigaspora margarita reveals the first report of DNA fragments corresponding to replicating non-retroviral RNA viruses in fungi. Environ. Microbiol., 20, 2012-2025.

Vandenkoornhuyse, P., Ridgway, K.P., Watson, I.J., Fitter, A.H. and Young, J.P.W. (2003) Co-existing grass species have distinctive arbuscular mycorrhizal communities. Mol. Ecol., 12, 3085-3095.

Varga, S. and Soulsbury, C.D. (2018) Arbuscular mycorrhizal fungi change host plant DNA methylation systemically. Plant Biol., 312, 599-600.

Vauthrin, S., Mikes, V., Milat, M.L., Ponchet, M., Maume, B., Osman, H. and Blein, J.P. (1999) Elicitins trap and transfer sterols from micelles, liposomes and plant plasma membranes. Biochim. Biophys. Acta, 1419, 335-342.

Venkateshwaran, M., Jayaraman, D., Chabaud, M., et al. (2015) A role for the mevalonate pathway in early plant symbiotic signaling. Proc. Natl. Acad. Sci. U. S. A., 112, 9781-9786.

Verbon, E.H., Trapet, P.L., Stringlis, I.A., Kruijs, S., Bakker, P.A. and Pieterse, C.M. (2017) Iron and immunity. Annu. Rev. Phytopathol., 55, 355-375.

Vernié, T., Camut, S., Camps, C., et al. (2016) PUB1 interacts with the receptor kinase DMI2 and negatively regulates rhizobial and arbuscular mycorrhizal symbioses through its ubiquitination activity in Medicago truncatula. Plant Physiol., 170, 2312-2324.

Vert, G., Grotz, N., Dédaldéchamp, F., Gaymard, F., Guerinot, M. Lou, Briat, J.-F. and Curie, C. (2002) IRT1, an Arabidopsis transporter essential for iron uptake from the soil and for plant growth. Plant Cell, 14, 1223-1233.

Voß, S., Betz, R., Heidt, S., Corradi, N. and Requena, N. (2018) RiCRN1, a crinkler effector from the arbuscular mycorrhizal fungus Rhizophagus irregularis, functions in arbuscule development. Front. Microbiol., 9, 1-18.

Wagner, G.P., Kin, K. and Lynch, V.J. (2012) Measurement of mRNA abundance using RNA-seq data: RPKM measure is inconsistent among samples. Theory Biosci., 131, 281-285.

Wang, E., Yu, N., Bano, S. a., et al. (2014) A H+-ATPase that energizes nutrient uptake during mycorrhizal symbioses in rice and Medicago truncatula. Plant Cell, 26, 1818-1830. 
Wang, W., Shi, J., Xie, Q., Jiang, Y., Yu, N. and Wang, E. (2017) Nutrient exchange and regulation in arbuscular mycorrhizal symbiosis. Mol. Plant, 10, 1147-1158.

Wang, Y and Wang, Y (2018) Phytophthora sojae effectors orchestrate warfare with host immunity. Curr. Opin. Microbiol., 46, 7-13.

Wang, Z., Ruan, W., Shi, J., et al. (2014) Rice SPX1 and SPX2 inhibit phosphate starvation responses through interacting with PHR2 in a phosphate-dependent manner. Proc. Natl. Acad. Sci. U. S. A., 111, 14953-14958.

Waters, M.T., Gutjahr, C., Bennett, T. and Nelson, D.C. (2017) Strigolactone signaling and evolution. Annu. Rev. Plant Biol., 68, 291-322.

Watts-Williams, S.J., Emmett, B.D., Levesque-Tremblay, V., MacLean, A.M., Sun, X., Satterlee, J.W., Fei, Z. and Harrison, M.J. (2019) Diverse Sorghum bicolor accessions show marked variation in growth and transcriptional responses to arbuscular mycorrhizal fungi. Plant Cell Environ., 42, 1758-1774.

Wawra, S., Fesel, P., Widmer, H., et al. (2016) The fungal-specific $\beta$-glucan-binding lectin FGB1 alters cell-wall composition and suppresses glucan-triggered immunity in plants. Nat. Commun., 7, 13188.

Wawra, S., Trusch, F., Matena, A., et al. (2017) The RxLR motif of the host targeting effector AVR3a of Phytophthora infestans is cleaved before secretion. Plant Cell, 29, 1184-1195.

Weiberg, A., Wang, M., Lin, F.M., Zhao, H., Zhang, Z., Kaloshian, I., Huang, H. Da and Jin, H. (2013) Fungal small RNAs suppress plant immunity by hijacking host RNA interference pathways. Science (80. )., 342, 118-123.

Werner, G.D.A., Cornwell, W.K., Sprent, J.I., Kattge, J. and Kiers, E.T. (2014) A single evolutionary innovation drives the deep evolution of symbiotic N2-fixation in angiosperms. Nat. Commun., 5, 4087.

Wewer, V., Brands, M. and Dörmann, P. (2014) Fatty acid synthesis and lipid metabolism in the obligate biotrophic fungus Rhizophagus irregularis during mycorrhization of Lotus japonicus. Plant J., 79, 398-412.

White, J., Prell, J., James, E.K. and Poole, P. (2007) Nutrient Sharing between Symbionts. Plant Physiol., 144, 604-614.

Whiteside, M.D., Garcia, M.O. and Treseder, K.K. (2012) Amino acid uptake in arbuscular mycorrhizal plants. PLoS One, 7, 8-11.

Whiteside, M.D., Werner, G.D.A., Caldas, E.A., Shimizu, T.S., West, S.A., Toby, E. and Correspondence, K. (2019) Mycorrhizal fungi respond to resource inequality by moving phosphorus from rich to poor patches across networks. Curr. Biol., 29, 2043-2050.

Wieczorke, R., Krampe, S., Weierstall, T., Freidel, K., Hollenberg, C.P. and Boles, E. (1999) Concurrent knock-out of at least 20 transporter genes is required to block uptake of hexoses in Saccharomyces cerevisiae. FEBS Lett., 464, 123-128. 
Winkelmann, G. (2017) A search for glomuferrin: a potential siderophore of arbuscular mycorrhizal fungi of the genus Glomus. BioMetals, 30, 559-564.

Wit, P.J.G.M. de, Burgt, A. van der, Ökmen, B., et al. (2012) The genomes of the fungal plant pathogens Cladosporium fulvum and Dothistroma septosporum reveal adaptation to different hosts and lifestyles but also signatures of common ancestry. PLoS Genet., 8, e1003088.

Xie, X., Lin, H., Peng, X., et al. (2016) Arbuscular mycorrhizal symbiosis requires a phosphate transceptor in the Gigaspora margarita fungal symbiont. Mol. Plant, 9, 1583-1608.

Xuan, Y.H., Hu, Y.B., Chen, L.-Q., Sosso, D., Ducat, D.C., Hou, B.H. and Frommer, W.B. (2013) Functional role of oligomerization for bacterial and plant SWEET sugar transporter family. Proc. Natl. Acad. Sci. U. S. A., 110, E3685-E3694.

Xue, L., Cui, H., Buer, B., Vijayakumar, V., Delaux, P.-M., Junkermann, S. and Bucher, M. (2015) Network of GRAS transcription factors involved in the control of arbuscule development in Lotus japonicus. Plant Physiol., 167, 854-871.

Xue, L., Klinnawee, L., Zhou, Y., et al. (2018) AP2 transcription factor $\mathrm{CBX} 1$ with a specific function in symbiotic exchange of nutrients in mycorrhizal Lotus japonicus. Proc. Natl. Acad. Sci. U. S. A., 115, E9239-E9246.

Yamaguchi, Y.L., Ishida, T. and Sawa, S. (2016) CLE peptides and their signaling pathways in plant development. J. Exp. Bot., 67, 4813-4826.

Yano, K., Yoshida, S., Müller, J., et al. (2008) CYCLOPS, a mediator of symbiotic intracellular accommodation. Proc. Natl. Acad. Sci. U. S. A., 105, 20540-20545.

Yu, N., Luo, D., Zhang, X., et al. (2014) A DELLA protein complex controls the arbuscular mycorrhizal symbiosis in plants. Cell Res., 24, 130-133.

Zamioudis, C., Hanson, J. and Pieterse, C.M.J.J. (2014) B-Glucosidase BGLU42 is a MYB72-dependent key regulator of rhizobacteriainduced systemic resistance and modulates iron deficiency responses in Arabidopsis roots. New Phytol., 204, 368-379.

Zeng, T., Holmer, R., Hontelez, J., et al. (2018) Host- and stagedependent secretome of the arbuscular mycorrhizal fungus Rhizophagus irregularis. Plant J., 94, 411-425.

Zhang, L., Feng, G. and Declerck, S. (2018) Signal beyond nutrient, fructose, exuded by an arbuscular mycorrhizal fungus triggers phytate mineralization by a phosphate solubilizing bacterium. ISME J., 12, 2339-2351.

Zhang, L., Xu, M., Liu, Y., Zhang, F., Hodge, A. and Feng, G. (2016) Carbon and phosphorus exchange may enable cooperation between an arbuscular mycorrhizal fungus and a phosphate-solubilizing bacterium. New Phytol., 210, 1022-1032. 
Zhang, Q., Blaylock, L. a and Harrison, M.J. (2010) Two Medicago truncatula half-ABC transporters are essential for arbuscule development in arbuscular mycorrhizal symbiosis. Plant Cell, 22, 1483-1497.

Zhang, X, Dong, W., Sun, J., Feng, F., Deng, Y., He, Z., Oldroyd, G.E.D. and Wang, E. (2015a) The receptor kinase CERK1 has dual functions in symbiosis and immunity signalling. Plant J., 81, 258267.

Zhang, X, Pumplin, N., Ivanov, S. and Harrison, M.J. (2015b) EXO70I is required for development of a sub-domain of the periarbuscular membrane during arbuscular mycorrhizal symbiosis. Curr. Biol., 25, 2189-2195.

Zhao, S., Chen, A., Chen, C., Li, C., Xia, R. and Wang, X. (2019) Transcriptomic analysis reveals the possible roles of sugar metabolism and export for positive mycorrhizal growth responses in soybean. Physiol. Plant., 166, 712-728.

Zipfel, C. and Oldroyd, G.E.D. (2017) Plant signalling in symbiosis and immunity. Nature, 543, 328-336. 


\section{Summary}

Arbuscular mycorrhizal (AM) fungi are able to form a symbiotic interaction with the vast majority ( $>80 \%$ ) of land plants. This interaction can benefit host plants in different ways; by providing nutrients (such as phosphate, nitrogen, and micronutrients), enhancing (a)biotic stress resistance of the host, and by improving soil structure and its associated microbiome. AM fungi colonize plants in a successive way, developing several stages including extraradical mycelium (ERM), intraradical mycelium (IRM) and arbuscules. Arbuscules are highly branched, tree-like structure surrounded by a specialized host membrane where specific nutrients transporters, are located to facilitate nutrient exchange. In general, arbuscules were considered as the heart of the symbiosis.

AM symbiosis is thought to have originated more than 400 million years ago. Such an ancient origin and wide-spread conservation suggest that both the hosts and AM fungi co-evolved to maintain the symbiosis. However, the molecular mechanisms that regulating this extremely high compatibility remain largely unknown. In this thesis, I tried to unravel some of the mechanisms that control the establishment of a compatible symbiotic interaction by both the plant and the fungus.

AM fungi have common fungal cell wall components which can be perceived as microbe-associated molecular patterns (MAMPs) that trigger immune responses. However, only minor plant immune responses are induced in early stage of AM symbiosis then rapidly suppressed. This suggests that AM fungi have highly efficient strategies to suppress host immune responses in a wide range of plants. Pathogenic microbes can suppress host immunity by secreting effector proteins that function either in the apoplast or that translocate into the host cells. As hundreds of putative effectors were discovered in the Rhizophagus irregularis genome, I hypothesized that some of those effectors might be essential for AM fungi to suppress host immunity, to remodel host structure, or to manipulate host metabolism to facilitate the symbiosis and consequently lead to an extremely high fungal compatibility. To discover essential AM effectors, I studied the AM fungal transcriptome in Medicago truncatula (Medicago), Nicotiana benthamiana (Nicotiana) and Allium schoenoprasum (Chives) in Chapter 2. This revealed a core set of AM effectors that are highly expressed in all three, evolutionary distantly related hosts as well as a set 
effectors that are expressed in a host-dependent manner. To determine at which stage the different effector candidates are expressed, I used laser microdissection in combination with RNA-Seq to profile the ERM, IRM and arbuscules in Medicago roots (Chapter 2). This showed that most host-dependent effectors are specifically induced in planta, suggesting an important role for AM effectors on controlling host-range (Chapter 2). By combining the host- and stage-dependent transcriptome data, I finally generated an expression landscape of all AM genes (Chapter 2 and 3) in different hosts and developmental stages, providing a valuable basis for future functional studies.

Among the effector candidates that are highly expressed upon intraradical colonization and in all three host plants, I found an effector carrying a single LysM domain (RiSLM, Chapter 2 and 4). Such LysM effectors from pathogenic fungi are famous for their ability to bind chitin and can suppress chitin-triggered plant immunity and/or protect the fungal cell wall against plant chitinases. I hypothesized that the RiSLM may have a similar function. We confirmed that RiSLM can indeed bind chitin and protect against the action of plant chitinases (Chapter 4). RiSLM binds long-chain $\mathrm{COs}$ (chito-oligosaccharides) with higher affinities than short-chain COs as shown by microscale thermophoresis (Chapter 4). As long-chain COs/chitin are strong elicitors for plant immunity, we tested whetherRiSLM can interfere with chitin-triggered immune responses. Our bioassays showed that RiSLM can efficiently suppress CO8-induced plant immune responses (Chapter 4 ). Although symbiotic signals such as LCOs (lipo-chitooligosaccharides) or short-chain COs are chitin-derivates that are also bound by RiSLM, RiSLM did not strongly interfere with sulfated LCO or CO4-induced symbiotic signalling (Chapter 4). In line with a role in subverting chitin triggered immune responses, we showed that host-induced gene silencing of RISLM reduced fungal colonization and arbuscule abundance (Chapter 4). Taken together, these results indicate that RiSLM plays an important role to subvert chitin-triggered immune responses to allow a successful AM symbiosis.

To get a broader insight into the transcriptional responses of the fungus to different host plants and at different developmental stages I analysed our host- and stage- dependent transcriptome data using gene ontology enrichment analyses (Chapter 3 ). Based on this data I identified several potential key fungal transporters that control nutrient transport at 
different stages of the interaction (Chapter 3). Furthermore, the hostdependent transcriptome profiles suggest a key effect of host identity on fungal secondary metabolism (Chapter 3 ).

The extremely ancient origin of AM symbiosis together with its broad hostrange implies that both partners co-evolved to maintain the symbiosis. In this scenario, it is essential that both partners benefit from the symbiosis, otherwise tit would be selected against and the symbiotic interaction would not have been maintained for such a long time. It is known that AM fungi obtain fatty acids and sugars (especially glucose) from the hosts, in return for mineral nutrients such as phosphate. However, the relative contribution or importance of both carbon sources is still unknown. Therefore, insight into the components that control nutrient exchange are required. Putative fatty acid transporters were previously identified, however, the sugar transporters that mediate sugar export from the plant to $A M$ fungi were still unknown. In my laser microdissection data, one arbuscule-induced Medicago SWEET (SUGARS-WILL-EVENTUALLY-BESECRETED) transporter, called MtSWEET1b, potentially involved in sugar export, stood out as candidate sugar exporter (Chapter 5). Functional analyses showed that MtSWEET1b is able to transport glucose (Chapter 5). In addition, MtSWEET1b localized to the peri-arbuscular membrane, where it is well suited to export glucose into the peri-arbuscular space (Chapter 5). Furthermore, overexpression of MtSWEET1b enhanced AM colonization, while expression of dominant-negative versions impaired arbuscule maintenance (Chapter 5 ). Taken together, these results suggest a (redundant) role for MtSWEET1b in the transport of glucose across the peri-arbuscular membrane to maintain arbuscules for a healthy mutually beneficial symbiosis.

By combining cell-specific transcriptome analyses with molecular functional approaches I was able to shed new light on some of the mechanisms that contribute to the broad host-range of AM fungi in light of effector biology and reciprocal nutrient transfer. Furthermore, the datasets generated and described in this thesis offer a valuable resource to deepen our understanding of the ecologically and agriculturally key AM symbiosis in the future. 


\section{Acknowledgements}

Time flies! Six years have passed since I arrived at Wageningen for the first time and now with this thesis, I finally come to the end of my PhD. I have to say that the five years in Wageningen was the best time of my life. Here, I would like to acknowledge all the people who helped or accompanied me in the past few years.

First, my supervisors. Erik, thanks for being my daily supervisor. I couldn't imagine how lucky I was to be able to work with you. Thanks for your guidance in my scientific development, your patience in my daily work, your encouragement upon my/our frustrations, and your suggestions for my career and life. I have looked up to you on: how to do science, how to solve problems, how to be a great supervisor and also a good friend to your PhD students, how to organize work efficiently, and how to balance work and life. I don't think I have learnt all of them but I shall not stop trying. And Ton, thanks for being my promoter. Thanks for allowing me to start my $\mathrm{PhD}$ in Wageningen with funding from the lab. Thanks for your help all through my PhD study. Thanks for all of the work discussion, advices, encouragements, and also the criticisms. I admire how you always consider training young scientists as one of the most important things. I admire your good knowledge, good logic, and your ability to always ask the most relevant questions. In addition to science, I was impressed by your skill for organization as well as administration. Ton and Erik, thanks for your help on my scientific training. I consider having you as my supervisors one of the luckiest things in my (almost) 30 years of life. When I came to Wageningen I was completely unsure about how to do science and whether I can do science. But now I am certain that I want to do science and I am confident to continue my scientific career. Wageningen has changed my life and I believe my Wageningen experience will benefit me lifelong.

Secondly, the mycorrhiza team: Jan $\mathrm{H}$, Rik, Jianyong, Peng, Jelle and people who did Bsc/Msc thesis in the mycorrhiza team especially Artem, Joran and Fangyuan. I have to say AM symbiosis is not easy to work with. Thank you for your assistance while fighting with all the troubles we had in our experiments. Thanks for your suggestions and comments for my projects. Rik, I am happy to have you as my paranymph on stage with me. It is nice that we can be paranymphs for each other. 
Third, I would like to thank all my colleagues in Wageningen. Maria and Marie-Jose, without such efficient secretaries the whole lab could not function properly. Marie-Jose, thanks for helping me to resolve many living/housing/subsidy problems when I had just arrived. Jan $\mathrm{V}$, Jan $\mathrm{H}$, Henk, Olga, Marijke, Carolien, and Sidney, thanks for your technical support/organization in the back to make the lab workable. Of course I also want to thank all lab members in Molbi, PDB or Cell Biology, Rene, Joan, Ben, Renze, Ikram, Viola, Elena, Wouter, Rens, Robin, Arjan, Yuda, Adam, Asma, Martinus, Titis, Lucas, Kavya, Jorge, Kana, Trupti, Simon, Luuk, Norbert, Han, Bandan, and Jeroen. Thanks for creating such a great working atmosphere and thanks for the enjoyable coffee/tea breaks we had together. I wish all of you a happy life and successful career.

It's difficult to live abroad; so far away from my family. I would like to thank the Chinese community in Wageningen which made me always feel like living with my family. 时间太快, 五六年勿勿而过, 六年前的瞬间似乎还 在昨天。在瓦大五年有太多跟大家在一起的回忆, 有些事虽然时间很久但是从未淡 去, 谢谢在瓦大的五年间有你们的陪伴。谢谢洁宇一直以来对我的支持和包容。谢 谢虎臣, 婷婷, 甲子哥, 卢媛, 文焜, 旭哥, 志纯, 罗露, 杜杜, 德峰, 凤娇, 标 标, 陈璐, 俊迪, 银山, 小白, 冯哥, 王璟, 莉莉, 郑琪, 明刚, 媛园, 陈正浩, 宋炜, 许萱, 宋银, 老毕, 材哥, 贵玲, 孟立, 䎦霞, 王艳, 杜羽, 锦斌, 金岭, 田卉, 朱峰, 涵子, 军伟, 赵涛, 老牛, 大钊, 温洁, 佳星, 付星, 薛飞, 子琳, 周琰, 馨元, 亦苑, 思瑜, 琳雅, 洪宇以及赵君老师, 成协设老师, 邓彦明老师。 还要感谢同样来自中农植物营养系圈子的小伙伴们。初来瓦大时和国华, 菁梦, 魏莎, 倩姐一起度过的无数个夜晚仍然历历在目, 也忘不了和勇哥每一次的畅饮以 及彻夜长谈。还有覃伟, 春旭, 金金鍂, 于洋, 明天, 齐超, 沐梵, 孟楚, 张朝春老 师, 东哥, 兆海, 程路, 丛汶峰, 荆晶莹, 苟芳, 俊奇。谢谢大家在瓦大给予我的 帮助。不管大家身在何处在做什么, 都希望大家一切顺利, 平安喜乐。最后要感谢 我的硕士导师申建波老师, 虽然已经离开了农大, 但是每次有机会跟申老师见面交 谈都会受益匪浅。感谢老师在我成长中给予的支持, 鼓励以及帮助。

Since January 2019, I started my new job in the Technical University of Munich, Germany. I also want to show gratitude to everybody in my current lab. Particularly Caro, thanks for your trust, patience, and inspiration. Thank you for letting me start before I finished my PhD. I am really happy to work with you and I really learnt a lot from you. I also want to thank all Gutjahr lab members: Daniela, Flo, Samy, Catarina, Chapy, Fan, Karen, Regina, Annika, Peter, Michi, Georg, Salar, Kartikye, Jose, Haifei, Karishma, Lotta, Nadine, Celia, Louis. Thanks for creating 
such a lovely and relaxed working environment. Thanks for your help and company in my daily life as well. I wish everybody a happy lab life and personal life.

In the end, I want to thank my family. 感谢我的家人, 谢谢你们在我成长路上 给予的支持和鼓励 !

$5^{\text {th }}$ Nov 2019

Freising, Germany 


\section{Curriculum Vitae}

Tian Zeng (曾添) was born on the $9^{\text {th }}$ of June, 1990 in Heze, Shandong Province, China. He studied Agronomy in Shandong Agricultural University from 2007-2011 and graduated from there with a bachelor's degree in Agronomy. Starting from September 2011, he was part of the group of Prof. Jianbo Shen at the Department of Plant Nutrition of China Agricultural University as a master's degree student. There he studied transporters mediating citrate secretion in cluster roots, using white lupin as a model plant. He started to be interested in plant nutritional biology particularly molecular mechanisms during his master's study. He graduated from China Agricultural University with a master's degree in Agronomy in the summer of 2013. In November 2013, he started his PhD at the Laboratory of Molecular Biology in Wageningen University, under supervision of Prof. Ton Bisseling and Dr Erik Limpens. In Wageningen, he investigated the mechanisms regulating fungal compatibility in arbuscular mycorrhiza symbiosis, focusing on effector biology and reciprocal nutrient transfer. Since January 2019, he joined the group of Prof. Caroline Gutjahr at the Technical University of Munich as a research associate. After his PhD, he will continue as a postdoc researcher in Prof. Gutjahr's group. 


\section{List of publications}

Zeng T, Holmer R, Hontelez J, Te Lintel-Hekkert B, Marufu L, Zeeuw T, Wu F, Schijlen E, Bisseling T and Limpens E (2018) Host- and stagedependent secretome of the arbuscular mycorrhizal fungus Rhizophagus irregularis. The Plant Journal 94: 411-425.

Cover paper: Highlighted by the Plant Journal: McCormick S (2018) An arbuscular mycorrhizal fungus adjusts its secretome depending on developmental stage and host plant. The Plant Journal 94: 409-410

An J, Zeng T, Ji C, de Graaf S, Zheng Z, Xiao TT, Deng X, Xiao S, Bisseling T, Limpens E, Pan Z (2019) A Medicago truncatula SWEET transporter implicated in arbuscule maintenance during arbuscular mycorrhiza symbiosis. New Phytologist 224: 396-408

Zeng T, Rodriguez Moreno L, Mansurkhodzhaev A, Wang P, van den Berg W, Gasciolli V, Cottaz S, Fort S, Thomma BPHJ, Bono JJ, Bisseling T, Limpens E (2019) A LysM effector subverts chitin-triggered immunity to facilitate arbuscular mycorrhizal symbiosis. New Phytologist, doi: 10.1111/nph.16245. 


\title{
Education Statement of the Graduate School
}

Experimental Plant Sciences

$\begin{array}{ll}\text { Issued to: } & \text { Tian Zeng } \\ \text { Date: } & \text { 10 December } 2019 \\ \text { Group: } & \text { Laboratory of Molecular Biology } \\ \text { University: } & \text { Wageningen University }\end{array}$

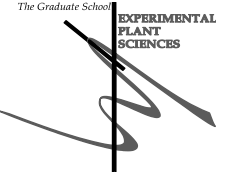

\author{
1) Start-Up Phase \\ - First presentation of your project \\ Mining the AM fungal genome for effectors in endosymbiosis \\ Writing or rewriting a project proposal \\ Writing a review or book chapter \\ MSc courses
}

\begin{tabular}{c|c|}
\multicolumn{1}{c|}{ date } & $\underline{c p}$ \\
17 Apr 2014 & 1.5 \\
& \\
\hline
\end{tabular}

2) Scientific Exposure

- EPS PhD student days

EPS PhD day, Leiden

EPS Get2Gether, Soest

EPS Get2Gether, Soest

EPS theme symposia

EPS theme 1 'Developmental Biology of Plants', Wageningen University

EPS theme 1 'Developmental Biology of Plants', Wageningen University

EPS theme 2 'Interactions between plants and biotic agents', University of Amsterdam

EPS theme 2 'Interactions between plants and biotic agents', Utrecht University

EPS theme 2 'Interactions between plants and biotic agents', Leiden University

EPS theme 2 'Interactions between plants and biotic agents', Wageningen University

EPS theme 3 'Metabolism and Adaptation', Wageningen University

EPS theme 3 'Metabolism and Adaptation', Wageningen University

EPS theme 4 'Genome Biology', Wageningen University

Lunteren Days and other national platforms

Annual meeting 'Experimental Plant Sciences', Lunteren

Annual meeting 'Experimental Plant Sciences', Lunteren

Annual meeting 'Experimental Plant Sciences', Lunteren

Annual meeting 'Experimental Plant Sciences', Lunteren

Annual meeting 'Experimental Plant Sciences', Lunteren

Annual meeting 'Experimental Plant Sciences', Lunteren

- Seminars (series), workshops and symposia

Seminar: Prof. Jiayang Li, Understanding the molecular mechanisms underlying rice tillering

Seminar: Prof. Jos Raaijmakers, Back to the Roots: exploring and exploiting the plant microbiome

Seminar: Prof. Jane Parker, Reprogramming cells for defence in plant innate immunity

Seminar: Prof. Sophien Kamoun, Genome and effector evolution in the Irish potato famine pathogen lineage

Seminar: Prof. Yuanchao Wang, Dissecting the interaction between Phytophthora sojae and soybean: making sense out of signalling and effectors

Seminar: Prof. Hanhui Kuang, Using the Nicotiana-TMV system to study resistance gene evolution and plant genome stability Seminar: Prof. Yves van de Peer, The evolutionary significance of gene and genome duplications

Seminar: Dr. Jiming Jiang, Structure and evolution of centromeres: lessons learned from plants

Seminar: Dr. Alain Goossens, How jasmonates provide the key to hamess plant chemistry

Seminar: Prof. Jane Parker, Plant intracellular immunity: evolutionary and molecular underpinnings

Seminar: Prof. Laura Grenville-Briggs, Molecular Oomycete-Host Interactions: The Good, the Bad and the ugly

Seminar: Dr. Jan Ruijter, Analysis of qPCR data. The use and usefulness of amplification curve analysis

Seminar: Prof. Douglas Mitchell, Genomics-enabled natural products discovery

Seminar: Prof. Mark Estelle, Auxin Signaling: Inputs and Outputs

Seminar: Dr. Pierre-Marc Delaux, Evolution of symbiotic gene networks in land plants

Seminar: Prof. Caitilyn Allen, How Ralstonia solanacearum succeeds in plant xylem vessels

Seminar: Dr. Jorge Casal, Signalling networks in plant responses to shade

Seminar: Dr. Kate Goodrich, The volatile 'language' of plants: from attraction to deterrence and back again

Seminar: Prof. Wenbo Ma, Effectors as molecular probes to understand pathogenesis

Seminar: Prof. Chun-Ming Liu, Rice nutritional improvement through modification of aleurone development

Seminar: Prof. Anne Osbourn, Hamessing plant metabolic diversity

Seminar: Dr. Sotirios Fragkostefanakis, Alternative splicing of a heat stress transcription factor mediates thermotolerance in tomato

Seminar: Prof.Hans Thordal-Christensen, Membrane trafficking in plant cells attacked by powdery mildew fungi

Seminar: Prof. Lars Østergaard, Fruit shape determination in Brassicaceae

Seminar: Dr. Annet Westhoek, Modelling resource allocation in the legume-rhizobium symbiosis

Seminar: Prof. Bregje Wertheim, Evolving immunity: Genomic basis of the evolution and variation in parasitoid resistance Seminar: Dr. Mart Krupovic, Natural history of viral capsids

Seminar: Prof. Ford Denison, Improving cooperation among plants and microbes

Seminar: Dr. Gerben van Ooijen, Clocks across taxa: Conserved cellular timekeeping mechanisms in plants, algae and other eukaryotes

Seminar: Prof. Marcel van der Heijden, Soil Biodiversity \& Ecosystem Functioning

Seminar: Dr. Martin Cann, The immune receptor Rx1 remodels chromatin and chromatin interactors in immunity

Seminar: Dr. Andrea Gröne, Why bother? Disease is nomal in wild life

Seminar: Prof. Peter Linder, Why is the Cape flora so species rich? Insights from the Cape reeds

Seminar: Dr. Jean-Francois Arrighi, Evolution of Nod factor-independent rizizobium symbiosis

Seminar: Prof. Giles Oldroyd, Recognition of symbiotic microorganisms by plants

Seminar: Prof. Janet Sprent, Updated classification of legumes and a discourse on Erythrophleum as an example of

continuing evolution in a basal legume clade.

Seminar: Dr. Euan James, Biogeography of legume nodulation by Beta-proteobacteria

Seminar: Prof. Sofie Goormachtig, Improving yield by understanding beneficial plant microbe interactions at the root-soil

interface

Seminar: Dr. Asaf Levy, Bacteria and the future of agriculture: from sequence to function

date

29 Nov 2013

29-30 Jan 2015

9-10 Feb 2017

24 Jan 2014

30 Jan 2018

25 Feb 2014

20 Feb 2015

22 Jan 2016

23 Jan 2017

14 Mar 2017

13 Mar 2018

13 Dec 2013

14-15 Apr 2014

13-14 Apr 2015

11-12 Apr 2016

10-11 Apr 2017

9-10 Apr 2018

8-9 Apr 2019

15 Nov 2013

07 Jan 2014

09 Apr 2014

28 May 2014

16 Jul 2014

11 Sep 2014

3 Feb 2015

1 Apr 2015

08 Dec 2015

21 Jan 2016

19 Feb 2016

14 Mar 2016

31 Mar 2016

7 Apr 2016

8 Apr 2016

29 Apr 2016

13 May 2016

23 May 2016

20 Jun 2016

21 Jun 2016

31 Aug 2016

2 Nov 2016

12 Dec 2016

21 Dec 2016

9 Jan 2017

19 Jan 2017

23 Feb 2017

8 May 2017

29 May 2017

15 Jun 2017

11 Jul 2017

28 Sep 2017

6 Oct 2017

18 Oct 2017

18 Oct 2017

23 Jan 2018

23 Jan 2018

23 Jan 2018

22 Feb 2018 
Seminar: Dr. Teemu Teeri, Pelargonidin in flowers - why not? Gerbera and petunia flowers block pelargonidin biosynthesis in a different way

Seminar: Ronald Snijder, Modern domestication of Pelargonium in a commercial environment

Seminar: Dr. Mary Wildermuth, Salicylic acid and cell cycle control of plant-microbe interactions

Seminar: Prof. Michael Djordjevic, CLE peptide dependent autoregulation of nodulation in Medicago truncatula

Seminar: Dr. Yan Wang, A leucine-rich repeat receptor-like protein as PAMP receptor recognising XEG1, a Phytophthora

glycoside hydrolase 12

Seminar: Dr. Diana Santelia, Rewiring starch metabolism for plant environmental adaptation

Seminar: Dr. Peter van der Esse, Mining NLRs from crop relatives to establish a diverse pool of disease resistance traits

Seminar: Prof. Ronald Pierik, Spotlight on shade responses: plant molecular strategies to cope with shade

Seminar: Prof. Hussam Hassan Nour-Eldin, The enigmatic substrate specificity of the NPF family - what is real - and what may be artifacts?

Seminar: Dr. Holger Schultheiss, Developing an integrated system for Asian Soybean Rust control

Seminar: Dr. Pilar Cubas, To grow or not to grow - a bud's question

Seminar. Prof. Julia Santiago, A tentative title could be: Plant cell wall communication and remodeling

Seminar. Prof. Ute Krämer, Plant acclimation and evolution in relation to soil mineral composition

Seminar: Dr. Andrea Sanchez Vallet, Communication between wheat and the necrotrophic pathogen Zymoseptoria tritici

Seminar: Dr. Brande Wulff, Of Crumbs and Bread: Understanding and exploiting immune receptor diversity in wild wheats

Seminar: Dr. Benoît Lacombe, Protein and gene regulatory networks involved in hormone-dependent nutrient sensing

Seminar: Prof. Ekkehard Neuhaus, Impact of sugar homeostasis on plant development and stress tolerance

Seminar: Dr. Martin Potocký, A well oiled machine: the role of anionic phospholipids in plant membrane traffic

Seminar. Prof. Stanislav Kopriva, Natural variation in nutrient homeostasis in Arabidopsis and beyond

Seminar: Prof. Andreas Schaller, Peptide signalling during plant reproductive development

Farewell symposium: Prof. Pierre de Wit, Fungal plant pathogens and the plant immune system

Farewell symposium: EPS director Prof. Ton Bisseling, The Undergrond Labyrinth: Roots, Friends and Foes

- Seminar plus

- International symposia and congresses

2nd International Molecular Mycorrhiza Meeting, Cambridge, UK

3rd International Molecular Mycorrhiza Meeting, Toulouse, France

4th International Molecular Mycorrhiza Meeting, Turin, Italy

- Presentations

Poster: Transcriptome analyses reveals potential host-specific AM effector proteins, 2nd Intemational Molecular Mycorrhiza Meeting, Cambridge, UK

Poster: Conserved LysM effector supresses chitin-triggered immunity to facilitate arbuscular mycorrhizal symbiosis, Annual meeting 'Experimental Plant Sciences', Lunteren

Talk: Host- and stage-dependent secretome of an arbuscular mycorrhizal fungus, Annual meeting 'Experimental Plant Sciences', Lunteren

Talk: Host- and stage-dependent transcriptome of Rhizophagus irregularis, 3rd International Molecular Mycorrhiza Meeting, Toulouse, France

Talk: A LysM effector subverts chitin-triggered immunity to facilitate arbuscular mycorrhizal symbiosis, 4 th International

Molecular Mycorrhiza Meeting, Turin, Italy

- IAB interview

Company visit: Keygene

Company visit: Dümmen Orange

14 Mar 2018

9 May 2018

25 Jun 2018

15 Aug 2018

10 Sep 2018

1 Nov 2018

10 Jan 2019

21 Jan 2019

17 Jan 2019

21 Feb 2019

28 Feb 2019

14 Mar 2019

21 Mar 2019

$28 \operatorname{Mar} 2019$

4 Apr 2019

11 Apr 2019

16 May 2019

23 May 2019

6 Jun 2019

4 Jul 2019

5 Jun 2014

8 Feb 2017

3-4 Sep 2015

26-28 Jul 2017

6-8 Feb 2019

3-4 Sep 2015

9-10 Apr 2018

11 Apr 2017

27 Jul 2017

7 Feb 2019

12 Oct 2017

15 Jun 2018

\section{3) In-Depth Studies}

3) In-Depth Studies
Advanced scientific courses \& workshops

Course: Genome assembly

Course: Introduction to R for statistical analysis

Course: Data analyses and visualization in $\mathrm{R}$

- Journal club

Journal club at Laboratory of Molecular Biology, Wageningen University

- Individual research training

4) Personal Development

- General skill training courses

Workshop: PhD Workshop Carousel 2015

Course: Techniques for writing and presenting a scientific paper

Course: Career orientation

Course: Last stretch of the $\mathrm{PhD}$ programme

Course: Reviewing a scientific paper

Course: Research data management (part 2 and 3 )

- Organisation of meetings, $\mathrm{PhD}$ courses or outreach activities

- Membership of EPS PhD Council

\begin{tabular}{|c|c|c|}
\hline date & $\underline{c p}$ \\
28,29 Apr 2015 & 0.6 \\
18,19 May 2015 & 0.6 \\
11,12 May 2017 & 0.6 \\
& $2013-2018$ & 3.0 \\
\hline
\end{tabular}

Subtotal In-Depth Studies

4.8

\begin{tabular}{|c|c|c|}
\hline date & $\underline{c p}$ \\
17 Apr 2015 & 0.3 \\
15-18 Mar 2016 & 1.2 \\
$3,10,17,24$ Oct 2017 & 1.5 \\
9 Jun 2017 & 0.0 \\
22 Jun 2017 & 0.1 \\
2,9 Nov 2017 & 0.3 \\
& & \\
\hline
\end{tabular}

TOTAL NUMBER OF CREDIT POINTS*

Herewith the Graduate School declares that the PhD candidate has complied with the educational requirements set by the Educational Committee of EPS with a minimum total of 30 ECTS credits. 
The research described in this thesis was performed at the Laboratory of Molecular Biology, Wageningen University, with financial support from the European Research Council (ERC-2011-AdG294790).

Financial support from Wageningen University for printing this thesis is greatly acknowledged.

Lay out: Tian Zeng

Cover design: Jieyu Liu, Tian Zeng

Print: GVO.nl 